University of Rhode Island

DigitalCommons@URI

Open Access Dissertations

1997

\title{
Variations in Attention as Measured by Accuracy and Reactions Time Over the Course of a Continuous Performance Task
}

Kevin J. Smith

University of Rhode Island

Follow this and additional works at: https://digitalcommons.uri.edu/oa_diss

\section{Recommended Citation}

Smith, Kevin J., "Variations in Attention as Measured by Accuracy and Reactions Time Over the Course of a Continuous Performance Task" (1997). Open Access Dissertations. Paper 937.

https://digitalcommons.uri.edu/oa_diss/937

This Dissertation is brought to you for free and open access by DigitalCommons@URI. It has been accepted for inclusion in Open Access Dissertations by an authorized administrator of DigitalCommons@URI. For more information, please contact digitalcommons-group@uri.edu. 
VARIATIONS IN ATTENTION AS MEASURED BY

ACCURACY AND REACTION TIME OVER THE COURSE OF A

CONTINUOUS PERFORMANCE TASK

BY

KEVIN J. SMITH

A DISSERTATION SUBMITTED IN PARTIAL FULFILLMENT.OF THE REQUIREMENTS FOR THE DEGREE OF DOCTOR OF PHILOSOPHY

IN PSYCHOLOGY

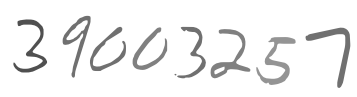

UNIVERSITY OF RHODE ISLAND 


\section{Abstract}

This study developed and examined new methods to identify and quantify moment to moment fluctuations in attention as measured during an auditory Continuous Performance Task (CPT). The study had three major components. The first was the investigation of methods to describe and quantify fluctuations of good performance in CPT data. The results of that investigation were two different techniques, one which examined the number and length of hit runs (one or more consecutive successful target detections) in the data, and the second which examined response data via a spectral analysis process. Where applicable, the methodologies were thoroughly tested to assess whether they would either introduce artifacts or distort real fluctuations. The positive results obtained during the examination and testing of these techniques were a strong indicator of the validity of these techniques for identification and description of fluctuations in CPT performance. The application of those techniques to real CPT data was the second major component. Twenty to twenty five minutes of archival CPT data from 40 participants was examined. The examination included description of the nature of runs of good performance (hit runs) and the identification and description of the presence and distribution of periodicity in the data. Differences between people of different competencies were also assessed, and two different dependent measures (accuracy and reaction time) were used where possible. General findings suggested that runs and periodicity were detectable in subject performance, and that there were minimal differences in the nature of these fluctuations between subjects of differing ability. The third component of this work comprised the validity testing of the techniques developed in earlier components. Methods for examining the 
origins of fluctuations in CPT performance were designed and implemented. The primary question addressed by these methods was, were the findings in component 2 attributable to some fluctuation in an attention mechanism, or were they due to some random factor or some artifact of test structure? The methodology involved the creation of 4000 simulated data sets by taking actual subject data and re-assigning hits and misses to different targets, thereby leaving total percent accuracy constant. Simulated data sets were matched to subject performance categories to minimize the difference between the number of errors in the function. Quantitative and rualitative comparisons between human and simulated subjects failed to provide firm evidence of differences between the two groups. Possible explanations for the results are discussed. 


\section{Acknowledgment}

To all of you who have poked prodded and supported me through this work I wish to express me sincere thanks.

To my wife Tara, whose love and patience have been without limits, I thank you from the bottom of my heart. To our loving pets Tika, Sebbie and Tasha, who have kept me company late into the night on so many occasions and who have sat and watched me work without question, I send my fondest thoughts.

To my parents Virginia and Nelson without whom I would never have endeavored down this path, I thank you for everything that I am. Your support has been of great value.

To my teacher and leader Dr. Dominic Valentino, whose support, diligence and wisdom have led me from despair and confusion to exultation and enlightenment, I wish you great thanks, and a hearty congratulations!

To Dr. Charles Collyer, Dr. Jerry Cohen, Dr. Susan Boatwright-Horowitz, Dr. Richard Vaccaro, Dr. Mark Kay, Dr. James Arruda, Dr. Sherri Gold, Mr. Peter Sutton and any others who I may have forgotten who have helped me to varying degrees over the long years of this project, I wish you my thanks.

To my committee members who endured multiple copies and long hours sifting through this material, I thank you.

1 dedicate this dissertation to my wife Tara and all of my relatives who have lived and labored before me. Their wisdom is not lost. Follow your dreams, and look not back at what might have been, but to what you are and where you are going... In the pursuit of knowledge, there are no losers. 


\section{Table of Contents}

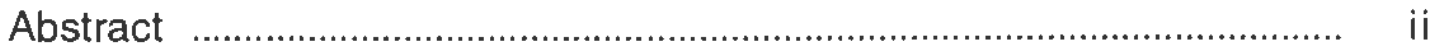

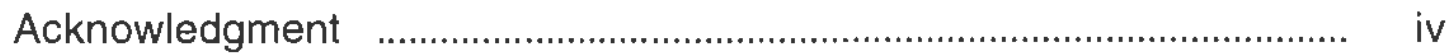

Table of Contents ….............................................................................. v

List of Tables ......................................................................................... ix

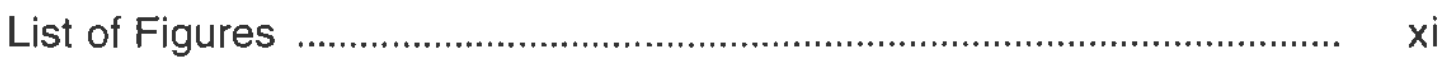

I. General Introduction .......................................................................

The Study of Attention ......................................................................... 2

The Vigilance Decrement.................................................................

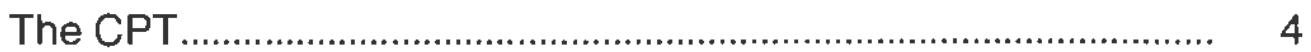

Recent Findings ..................................................................... 5

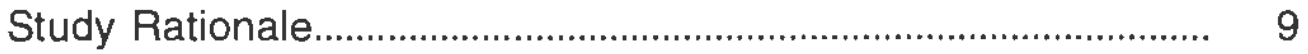

General Hypotheses \& Predictions ................................................. 10

II. General Methods ................................................................................ 11

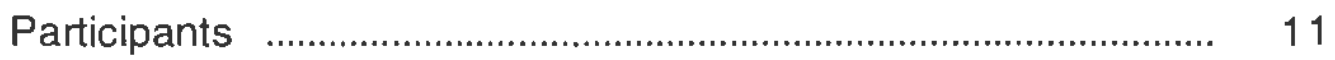

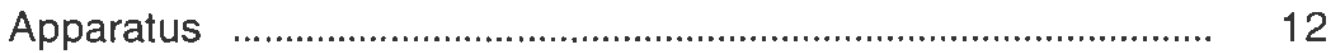

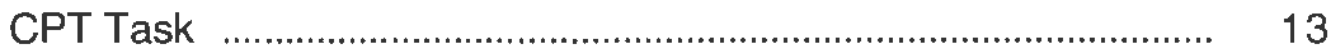

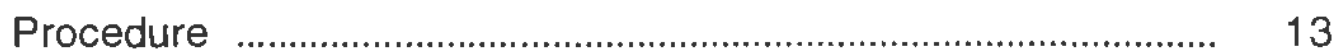

III. Introduction to Experiment 1 ................................................................ 15

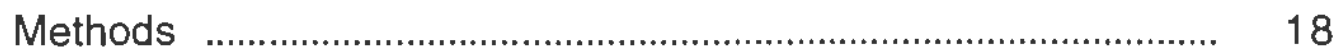

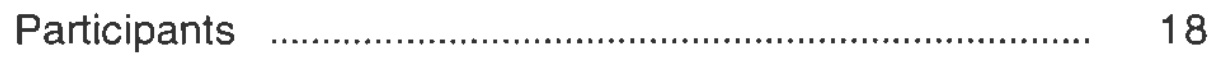

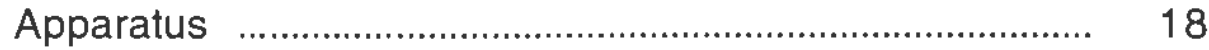

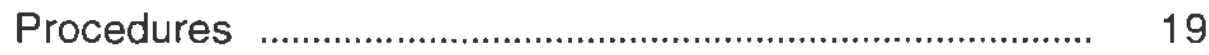

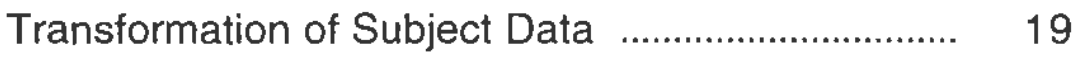


Results

Results

Discussion: Experiment 1 Parts $1 \& 2$

IV. Introduction to Experiment 2

Methods 30

Participants 30

Apparatus 30

Procedures 30

Experiment 2 Part 1 30

Results 33

Experiment 2 Part 2 34

Results 37

Discussion: Experiment 2 Parts $1 \& 2$

Experiment 2 Part 3 42

Results 44

Discussion: Experiment 2 Part 3 45

Experiment 2 Part 4 46

Results

Discussion: Experiment 2 Part 4

General Discussion: Experiment 2

V. Introduction to Experiment 3 50

Methods 
Experiment 3 Part 1 ............................................. 51

Results ..................................................... 52

Discussion: Experiment 3 Part 1 ................. 54

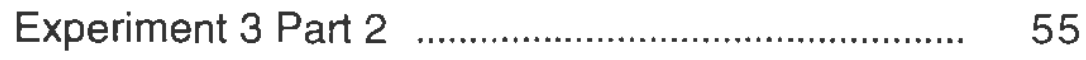

Results \& Discussion $\quad$................................... 56

Experiment 3 Part 3 .............................................. 61

Results ....................................................... 62

Discussion: Experiment 3 Part 3 ................. 62

General Discussion: Experiment 3 .............................................. 62

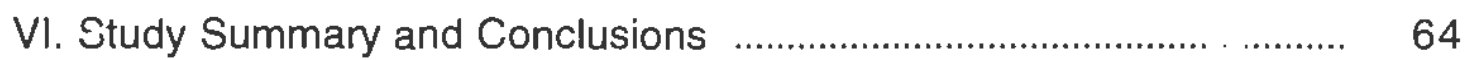

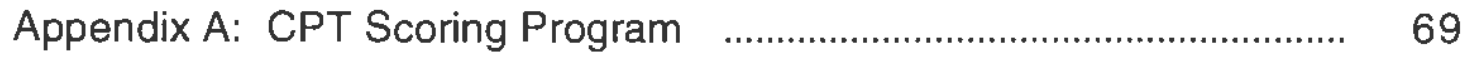

Appendix B: Simulation Target File _.................................................... 89

Appendix C: Experiment 1 Part 1 ANOVA Tables ….............................. 93

Appendix D: Experiment 1 Part 1 Chi Squares ........................................ 97

Appendix E: Experiment 1 Part 2 ANOVA Tables ................................... 99

Appendix F: Simulation Builder Program ………................................ 101

Appendix G: FFT with Hamming Window Program $\ldots \ldots \ldots \ldots \ldots \ldots \ldots \ldots \ldots \ldots \ldots . . . . . . . . . . . . .104$

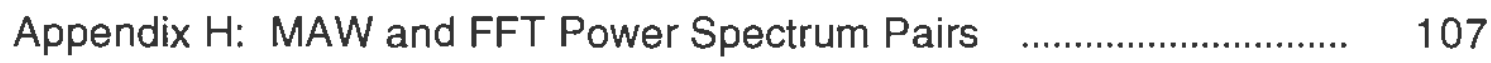

Appendix I: Experiment 2 Part 3 Chi Squares ......................................... 111

Appendix J: Experiment 2 Part 4 Chi Squares ...................................... 113

Appendix K: Experiment 3 Part 1 Chi Squares ...................................... 115

Appendix L: Accuracy vs. Reaction Time Power Spectra $\quad$.................... 117

Appendix M: Experiment 3 Part 3 Chi Squares ...................................... 158 
Tables

Figures

Bibliography 


\section{List of Tables}

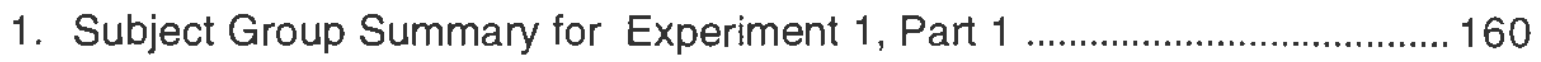

2. Subject Group Summary for Experiment 1, Part 2 ….................................. 161

3. Summary of Performance for all Subjects ............................................... 162

4. Summary of Monti-Carlo Group Information ............................................... 164

5. Average Number and Length (in Number of Targets) of Hit Runs by Group for Human \& Simulated Subjects 165

6. Average number of Hits and Misses following Hits at Short and Long Intervals and Ratios, Short to Long Interval 166

7. Means and SD of dependent measures for 15 top and 15 bottom performers

8. Summary of Characteristics for MAW Test Case Functions created in Experiment 2, Part 1

9. Primary Frequencies in Minutes per Cycle from the Accuracy FFT, Subjects with Best Power Only 169

10. Mean and SD in Minutes per Cycle and Percentage of Subjects with a Frequency in Each Bin for Human Subjects based on Original Bins ....170

11. Summary of Narrowed Bin ranges, and actual ranges for Human and Simulated Subjects in Minutes

12. Percentage of Human and Modeled Subjects as members of narrowed Bins

13. Percentage Membership for human subject groupings by FFT periodicity range bin 
14. Primary Frequencies in Minutes per Cycle from the Reaction Time FFT, Subjects with Best Power Only

15. Summary of Narrowed Bin ranges in Minutes per Cycle, and actual ranges for Human Subject (Accuracy \& Reaction Time)

16. Summary of Reaction Time and Accuracy FFT results in Minutes per Cycle and Percent of Subjects with a Peak in each Bin for Human Subjects based on Narrowed Bins

17. Percentage of Human Subjects Categorized for Clarity of Primary Frequency Power for Accuracy and Reaction Time 178

18. Overall Noise Level in Accuracy and Reaction Time Spectra for Human Subjects

19. Summary of the Comparability of Primary Frequency Peaks for Accuracy and Reaction Time Spectra 180

20. Percentage Membership for Human Subject Groupings by RT FFT Periodicity Range Bin 181 


\section{List of Figures}

1. The distribution of window sizes (number of targets falling within the 30 second window) for the MAW function on the CPT template

2. MAW Data Plot for Case 1. Hits (correct target detections) were arranged to occur with 2 minute periodicity (No noise) 183

3. MAW Data Plot for Case 4. Hits were arrange to occur with a 2 Minute Periodicity (High Noise) 184

4. FFT power spectrum for Case 1. Hit runs were arrange to occur with a 5 Minute Periodicity. Calculations of frequency periods in minutes are shown.

5. FFT power spectrum for Case 2. Hit runs were arrange to occur with a 5 Minute Periodicity with $20 \%$ noise. Calculations of frequency periods in minutes are shown.

6. FFT power spectrum for Case 3 . Hit runs were arrange to occur with a 5 Minute Periodicity with $40 \%$ noise. Calculations of frequency periods in minutes are shown.

7. FFT power spectrum for Case 4. Hit runs were arrange to occur with 1 and 5 Minute Periodicities. Calculations of frequency periods in minutes are shown. 188

8. MAW data FFT power spectrum for Case 1. Hit runs were arrange to occur with 2 Minute Periodicity. Calculations of frequency periods in minutes are shown. 
9. MAW data FFT power spectrum for Case 2. Hit runs were arrange to occur with 2 Minute Periodicity and a low level of noise was added.

Calculations of frequency periods in minutes are shown.

10. MAW data FFT power spectrum for Case 3. Hit runs were arrange to occur with 2 Minute Periodicity and a moderate level of noise was added. Calculations of frequency periods in minutes are shown.

11. MAW data FFT power spectrum for Case 4 . Hit runs were arrange to occur with 2 Minute Periodicity and a high level of noise was added. Calculations of frequency periods in minutes are shown.

12. MAW data FFT power spectrum for Case 5 . Hit runs were arrange to occur with 2 and 4 Minute Periodicities. Calculations of frequency periods in minutes are shown. 193

13. MAW data FFT power spectrum for Case 6 . Hit runs were arrange to occur with 1 and 4 Minute Periodicities. Calculations of frequency periods in minutes are shown. 194

14. MAW data FFT power spectrum for Case 7. Hit runs were arrange to occur with 1 and 5 Minute Periodicities. Calculations of frequency periods in minutes are shown.

15. FFT Power Spectrum showing a good example of high primary frequency clarity. 196

16. FFT Power Spectrum showing a good example for low primary frequency clarity. 197

17. FFT Power Spectrum showing a good example of an overall Low noise level on a power spectrum 
18. FFT Power Spectrum showing a good example of a high overall noise level in a power spectrum.

19. Power Spectra (RT and Accuracy) for Human Subject 48 , rated as Same for primary frequency bands.

20. Power Spectra (Accuracy and RT) for Human Subject 55, rated as Different for primary frequency bands. 201 


\section{Introduction}

In the psychophysiology laboratory at the University of Rhode Island, unique methodologies for the study of attention are being developed. Over a number of years, researchers at the laboratory have collected both neuroelectrical and behavioral data from participants as they performed a vigilance or sustained attention task, specifically an auditory continuous performance test (CPT). All subjects participating in these trials were closely screened for factors which might affect performance. Studies of quantified electroencephalogram data yielded positive results in validating a model containing seven to eight specific rieurocognative systems, including an attention component (Arruda, 1994; Arruda et al., 1996).

While many studies in the past 20 years have used sophisticated techniques for examining neurocognitive data collected during attention tasks, a strictly traditional evaluation of behavioral data has been followed by most researchers who choose to examine the behavioral elements of attention.

These traditional methods have most often included a simple globalor wideangle view of the behavioral data along the lines of two dependent variables, accuracy and response latency. The vast majority of such studies have observed a performance decrement from the beginning of the task to the end, which is measured by an increase in response latency and a decrease in accuracy, indicating a decrement in sustained attention over the course of the task (See et al., 1995). These methods have proved useful both in research and clinical settings. Recently, however, new research conducted by Makeig (1993), Gilden et. al. (1995b), Conte et. al. (1995), and theoretical treatments of 
attention by Tucker (1981), have explored the possibility that there may be more occurring than just a gradual loss of ability to attend. These authors suggest that performance may fluctuate in cycles or streaks over time.

The primary purpose of this study was to establish the nature of fluctuations in performance over the duration of a CPT, and to evaluate the results, in part, in contrast to a model of gradual decline in performance (the Performance Decrement). The origins of the study of attention, specifically sustained attention or vigilance, are discussed in the following section.

\section{The Study of Attention}

The questions surrounding the brain's ability to mediate an individual's perception of his/her surroundings were examined by such prominent names as William James and J. Hughlings Jackson during the nineteenth century. It was in the writings of Jackson that the concept of sustained attention appears. The concept of attention itself refers to the individual's ability to perceive objects and events in their environment. There are numerous and diverse models of the attention process, but at its root, it can be broken down into two subcategories, selective and intensive. It is in selective attention where our interest lies, because vigilance, a relatively new but key term in the study of attention, simply refers to sustained selective attention (Parasuraman, 1984). Thus, when we refer to vigilance, we refer to the ability of an individual to selectively maintain focused attention on an object or event in their environment over a long period of time.

The first use of the term vigilance in a technical sense was in the early twentieth century by Sir Henry Head, a British neurologist. In his work, Head 
defined vigilance as "A state of high grade efficiency of the central nervous system" (Warm, 1984), suggesting that the physiology of the mind was in an energized state of readiness. It was not Head, however, who primed the cannon of the scientific exploration of vigilance, but a man named Norman Mackworth. Mackworth, also a neurologist, conducted several studies of vigilance for the British Airforce. Their interest lay in the ability of an individual to monitor radar devices. Mackworth noted degradation in performance of the task over a period of time (Mackworth, 1948). Mackworth also modified the definition of vigilance as a "state of readiness to detect and respond to certain small changes occurring at random time intervals in the environment" (Warm, 1984). Mackworth's definition for vigilance was tailored to his studies of monitoring and watchkeeping, rather than the neurological bent of Head's definition. It was Mackworth's series of experiments, and similar experimentation on radar fatigue conducted by Lindsley (Parasuraman, 1984) which created the questions and the interest to launch the modern study of vigilance.

\section{Vigilance Decrement}

Perhaps the most pervasive and robust finding of the study of vigilance has come to be described as the vigilance decrement (Davies \& Parasuraman, 1982; Warm , 1984). Laboratory tests of monitoring/vigilance tasks have shown a marked decrease in detection of target stimuli beginning after as little as two to three minutes of the trial. Accuracy continues to decrease over time until it reaches a floor level of only 70 to $80 \%$ of initial level of accuracy. Similar results have been obtained in the lab at the University of Rhode Island. In a study 
examining the existence of a right hemisphere attention mechanism using an EEG and an auditory CPT task, Arruda (1994) noted an average decline in performance ranging from $15 \%$ error rate in the first five minutes of a twenty minute task, to $35 \%$ error rate in the last five minutes.

\section{The CPT}

The CPT itself was developed in the 1950's by Rosvold, Mirsky, Sarason, Bransome, and Beck (Davies \& Parasuraman, 1982). Rosvold et al. were interested in comparing the ability of brain-damaged and non-brain-damaged patients to sustain attention. The task that they created used a simple accuracy measure to assess performance. Although characteristics of the task have been manipulated, its basic methodology has remained the same. The CPT consists of a set of masking (non-target) stimuli presented at intervals, and a set of target stimuli presented either simultaneously or successively to the masking stimuli. Subjects make some response, such as a button press, when they detect a target. Several measures are traditionally assessed. HITS are correct responses to target stimuli within the allowable response latency time. MISSES are non-response to target stimuli. FALSE ALARMS are incorrect reponses to non-target stimuli. LATE HITS are responses to targets which occur outside of the allowable response latency, and REACTION TIME is a measure of response latency to hits.

Recent vigilance research has seen an increase in the popularity of the CPT. While this may be due in some part to the increasing availability of desktop computers which are the favored medium to administrate the tests, it is also due to the belief that the CPT assesses attention 'independent of verbal, perceptual, and other cognitive processing abilities' (Halperin et al., 1991). 
Halperin has studied the clinical utility of the CPT in great depth. Along with various other groups of researchers, Halperin et al. have attempted to demonstrate the ability of the CPT to discriminate children of clinical groups (i.e. ADHD) and normal controls (see O'Dougherty, Nuechterlein, \& Drew, 1984; Shaprio \& Garfinke, 1986; Swanson, 1981; and Halperin et al., 1988). Research conducted by Halperin and his collegues has allowed them to validate the CPT as a research tool for the study of attention. Halperin writes "Although the CPT has tremendous face validity as a measure of attention, this view must still be put forth with caution." Halperin et al. have developed three constructs which can be differentiated by error types. These constructs are inattention, impulsivity and dyscontrol. Measures are based on false alarms, misses, long latency responses and, to some degree, on the response reaction time. Their research into the clinical use of the CPT has led them to suggest that the CPT is a useful research tool and will likely play a strong role in the diagnosis of attentional dysfunction, but that at this time further research is required into the constructs assessed by the $\mathrm{CPT}$, and into the exact nature of the deficits to be identified (Halperin, et al., 1991).

\section{Recent findings regarding fluctuations in sustained attention}

A study by Makeig \& Inlow (1993) designed to investigate the correlations between performance and power in the EEG spectrum revealed an interesting result. Performance and EEG data were recorded from 13 male subjects as they performed simulated passive sonar detection tasks, two trials of 28 minutes each. Targets were presented pseudo-randomly at a rate of 10 per minute, in a continuous string of background signals occurring at intervals of 2-4 
seconds. Makeig and Inlow scrutinized their data and recorded a measure which they called Local Error Rate consisting of the fraction of non-detected targets in a moving time window. Their window was 32.8 seconds in length and was advanced along their data in 1.64 second steps. This procedure produced a continuous estimate of performance at regularly spaced time intervals which was necessary for comparison with EEG data. Makeig and Inlow used a MontiCarlo procedure to model random local error rate functions by "shuffling", randomly permuting, the order of detected and undetected targets and then applying the same windowing procedure applied to the real data. This procedure was repeated 200 times for each subject and the results were averaged to create a spectrum of expected values. Fourier spectral analyses were conducted for each real subject and expected data set. Differences between actual and expected error rate spectra were assessed, and it was determined that values larger than chance dominated the spectrum at cycle lengths longer than 4 minutes. No evidence for rhythmicity at any particular cycle length was noted.

Gilden \& Gray-Wilson conducted several experiments designed to assess the nature of streaks (runs of accurate performance) in skilled performance tasks and nonstationarity of responding. Their first set of experiments were signal detection studies (1995a), concerned not with threshold but rather with providing a way of examining nonstationarity in responding without the additional complexity of stimulus uncertainty and task difficulty. Each task presented stimuli at threshold due to the fact that at superthreshold levels discrimination would have been nearly effortless, and it is not possible to study outcome sequence structure without errors. Studies were 
conducted for both visual and auditory modalities, with multiple within subject trials, and with preattentive and attention-processed stimuli. Results were assessed in terms of numbers of observed runs (successive correct responses) as compared to results expected if sequences were derived from a Bernoulli process. Inferential statistics (ANOVA) revealed that preattentive tasks were streakier than tasks requiring focused attention. Theoretical analysis was also employed. Four models of nonstationarity were considered. Learning modeled a secular improvement or worsening over the course of a trial. Wave Modulation modeled streaks that might arise due to a wave-like fluctuations in attention, ability or effort. Intermittent Effort modeled two states of effort or ability that were distinguished by hit rate. Markov Process modeled correlation between successful trials. Models were assessed by Part-Serial correlation and Monti-Carlo simulation. Wave modulation was selected as the model which most closely resembled observed human performance.

In their second set of experiments, Gilden and Gray-Wilson (1995b) examined streakiness in skilled performance. Fluctuations in performance while performing golf putting and dart throwing were assessed via the same processes employed above. They found evidence of streaky performance in motor skills, and evidence for a U-shaped function relating streak magnitude and hit rate. As in the first set of studies, theoretical analyses revealed that a wave-modulation model best fit the actual human response data. Gilden and Gray-Wilson also noted that task difficulty was a determinant factor in the visibility of streaky performance. A task which was too easy or difficult masked streaks in performance due to the ceiling and floor effects of the dependent measure, hit rate. 
Conte, Ferlazzo and Renzi (1995) conducted a study meant to examine the presence of rhythmic fluctuations in vigilance tasks. They were looking for rhythmic variation in performance beyond a linear decrease over time (the performance decrement). To begin, they employed two tasks, one which presented a steady stimulus at 20 second intervals, and one which presented a stimulus at 40 second intervals, while interjecting a masking stimulus at quasjrandom intervals between the 40 second targets. In both tasks subjects responded to all stimuli with a button press. Reaction time was recorded. Reaction time data from masking stimuli was discarded. The second task was adopted due to the fact that subjects might forecast occurrence of the stimuli. 37 subjects performed the first task for 60 minutes. 10 subjects performed the second task for 85 minutes. Discrete Fourier Transformation algorithms were used to compute power spectra. Spectra were smoothed by means of a Parzen lag window. One sample Kolmogorov-Smirnov tests were used to determine if each spectrum was significantly different from white noise. Spectral density values above 2.81 SDs were considered significant for identifying peaks. For task 1,34 of 37 subjects showed performance which was significantly different from a random process, and 31 subjects showed significant peaks with periods ranging from 5 to 30 minutes. For task 2,9 of 10 subjects showed performance which was significantly different from a random process, and 8 subjects showed significant peaks with periods ranging from 5 to 28 minutes. Two subjects who participated in both tasks showed comparable significant peak frequencies (subject $A, 7.3$ and 7 minutes; subject $B, 30$ and 28 minutes). Conte et. al. suggested that the results of these studies indicated the presence of an ultradian rhythm in attentional capacity, and that this rhythm was not linked to 
regularity in the presentation of stimuli. They went on to suggest that the results cannot indicate whether or not the independence of stimulus presentation rate and performance is absolute, or if that, in some conditions, the type and duration of stimulation might effect performance. As a final step in their investigation, Conte et. al. conducted a study designed to show within-subject stability with respect to performance. 5 new subjects were recruited, and each were tested twice on separate days using the task 2 procedures. Coherence (frequency domain correlation) values were calculated for pairs of subject spectra. For 4 of 5 subjects, there was a good match between the maximum power peaks of both spectra. Coherence values for all subjects were quite high, .45 to .75 . Rhythmic variations in RT were found to have a periodicity from 9.48 to 28.44 minutes in all subjects. The results of the third study would seem to preclude the possibility of an artificial nature for observed periodicity.

\section{Rationale for this Study}

Despite decades of study, the nature of attention is still elusive. A closer examination of the apparent cyclical nature of performance could yield a three fold benefit. First, it could suggest a basis for a new and more revealing methodology for examining vigilance data. The present study has developed methods for detecting fluctuations and periodicity in hit rate and reaction time as dependent variables for assessing performance, and attempts to reveal which is a more sensitive measure. It examines the moving average filter as a tool for data analysis of CPT data as well as two different methods for measuring periodicity. 
Second, such study could provide valuable information to applied scientists. Identification of a new and potentially more sensitive measure of performance which has been correlated with attention would allow the creation of new measures and screening devices for attentional disorders, and perhaps allow more specialized categorization by way of normative data.

The third area of potential gain is that of theory. Attention is such a complicated process, or set of processes that it has been nearly impossible to create a model which seems to emulate all characteristics (Warm, 1984). This study evaluates the data in light of the traditional model of gradual decline of performance, and also suggests other plausible models and directions for further investigation.

\section{Hypotheses \& Predictions:}

The general hypotheses explored in this dissertation are that 1) our ability to sustain attention over time fluctuates, perhaps oscillates with a regular periodicity, and that 2) the oscillations can be measured in CPT performance if the proper methodology (including the appropriate CPT design) is developed.

CPTs have good face validity as tasks that require sustained attention and are widely used to assess it. However, in the few studies of sustained attention which attempted to investigate moment-to-moment variations, vigilance appeared to fluctuate over the course of a long task. A picture of these fluctuations would be lost in conventional CPT performance analyses. CPT targets usually occur at a rate of only 2 or 3 per minute at most, and performance is reported as percent accuracy (or mean reaction time) over the entire period of the task or as a performance (or reaction time) decrement 
between some early task period and a later one.

The psychophysiology lab has already collected over 20 minutes of CPT responses for each of a large number of subjects. This CPT presents target stimuli (probing attention) at an average inter-target interval of 6 seconds, a much denser target rate than almost any other CPT previously used. Furthermore, targets were defined as two consecutive identical stimuli, as opposed to the occurrence of some particular stimulus. Thus, good performance depends upon processing each and every stimulus, i.e. it demands continuous controlled attention.

The general predictions of this work were that 1) CPT performance data of 40 subjects will be adequate to develop measures that will approximate momentary levels of performance (i.e., attention), and that 2) these analyses will reveal that performance generally declines in periodic oscillations of good and poor accuracy or of rapid and slower response latency.

\section{General Methods}

\section{Participants}

Fifty-one participants were selected from an archival data set. All subjects were recruited from a general psychology course (PSY113) or a junior level perception course (PSY385) at the University of Rhode Island. Participants received course credit for their participation in this data collection. All participants reported that they were free of neurological conditions. The protocol used to collect this subject data was reviewed and accepted by the University of Rhode Island Human Subjects Review Board. 
It was decided that, to avoid outliers in the data, certain subjects should be removed from the study. Those were subjects whose False Alarm Rate was greater than .025 , suggesting lack of effort in doing the task, and subjects constituting the top and bottom $5 \%$ of all subjects based on the accuracy of their performance.

Selection of subjects meeting these criteria was made following initial analysis of the subject data. After subjects falling in to these classes were removed, 40 subjects remained ( 13 men and 27 women), and were used for the remainder of the study. Although age data was not available for all subjects, the age range for those known of the 40 remaining subjects was 18 to 24 years, with a mean of $18.47(\underline{\mathrm{SD}}=1.22)$ years. All but one subject was right handed as assessed by a modified version of the Edinburgh Handedness Inventory (Olfield, 1971).

\section{Apparatus}

An IBM compatible $80486 \mathrm{D} \times 2-66$ computer was used to present the stimuli and record accuracy and response latency for the continuous performance task. Participants listened to stimuli via headphones. Responses were made by depressing a button on a hand held actuator. A second IBM style computer running a free UNIX operating system (LINUX) was used to model data, process data for further analysis, and to summarize data. A Macintosh computer running SPSS-MAC, Microsoft Excel 5.0a and Matlab 4.0 software packages was used for data analysis and figure creation. 


\section{CPT Task}

All subjects were run through a single identical auditory CPT condition designated as Letters. Letters was a phonetic task consisting of 24 alphabetic letter names presented in quasi-random order at a rate of 2 per second for 20 25 minutes. A target was defined as two consecutive presentations of the same letter. Targets occurred approximately ten times a minute, at an average interval of 6.51 seconds. The maximum and minimum time between targets were 14 and 2 seconds respectively.

\section{General Procedure}

Upon entering the laboratory, the subject was given an informed consent form, and a set of questionnaires to sign and fill out. Questionnaires probed medical history, handedness and mood. When each had completed the paperwork, they sat in a comfortable arm-chair while electrodes were applied to their scalp. This part of the procedure took between twenty-five and forty-five minutes. Once all electrodes were applied, the subject was given the following instructions:

For the first phase of this experiment we ask that you sit quietly with your eyes closed, your arms in your lap and your legs extended outward. After resting for a period of approximately three minutes we will ask that you perform a task which will constitute the second phase of our experiment. The task will require that you listen to a series of computer generated letters of the alphabet through headphones. The letters are spoken randomly, one immediately following the other. It will be your job to press the button once, using your right hand, for each time you hear the same letter spoken twice consecutively (e.g., $d \mathrm{~d}$ within the sequence $a \mathrm{~b} d \mathrm{~d} k$ ). Please keep your eyes closed throughout the entire procedure (both phases) and try not to move in your seat. We will verbally signal you when you are about to begin the second phase. You will be notified when you have completed the experiment. We would like you to wear the 
headphones and hold the button during the first phase of the experiment. Do you have any questions before we begin?

At this point, the participants rested for approximately two minutes and forty five seconds while a sample of QEEG was taken. When the rest phase was over, the participants were informed that the second phase would begin. The participants then performed the CPT for as much as twenty five minutes. Mean trial length was 21 minutes. Actual length of trial was determined by the amount of clean EEG gathered during the trial. The EEG data was not examined in this study. Accuracy and response latency data was collected by the computer. When data collection was complete, the data was studied and then archived.

Please refer to individual Experiments for a description of experiment specific procedures. 


\section{Experiment 1}

\section{Variation in Attention: Is accurate responding random over time?}

A pilot experiment was conducted with 10 human data sets to test general methodological procedures, and to confirm that fluctuations in performance were evident in CPT data. Various visual representations of the data were created and examined qualitatively. Fluctuations in responding were revealed. Motivated by a need for a tool to observe momentary changes in attention, it becomes important to identify the nature of those fluctuations. The first task was to identify a methodology which would differentiate fluctuations in accurate responding caused by random processes, from those resulting from non-random processes.

Examination of related literature revealed that only a single team of researchers was working on related projects. Gilden and Gray-Wilson (1995b) have conducted several experiments looking for streaks in skilled performance. They defined streaks as two or more consecutive correct or accurate responses or actions. Their interest revolved primarily around the idea that there is a perception among athletes, fans and anyone else who may be practicing a skilled activity, that "there are moments when a person is hot, or in the zone, or alternatively is cold, or in a slump." They relate this to the notion of flow which they define as the "dimension of experience where the intimate coupling of actor and activity results in a specific psychological state anecdotally referred to as being hot." They went on to posit that the flow state or streaky performance arises from some aspect of the skilled activity, or alternately from mistaking chance fluctuations for heightened ability. Such miss-attributions have been studied and well documented. Study has shown that people have systematic 
biases which prevent them from accurately differentiating random from nonrandom. Consider the following statement. Since a quarter has landed on heads three times in a row, it is time for it to land on tails. Despite the run or steak of heads, the probability of the quarter landing on heads or tails remains the same with every coin toss. People, however, tend to attribute an almost magical quality or fate to the process, and might be more likely to bet that tails will follow on the next flip. (See Tversky \& Kahneman, 1971, 1974, for additional information.)

Gilden and Gray-Wilson (1995b) addressed the following question. Is the serial execution of a skilled action distinguishable from a Bernoulli process when the playing conditions are identical on all trials? In other words, do hits beget hits? If so, then there would be fewer runs of hits than expected under the null hypothesis of Bernoulli trials indicating more internal repetition and a positive serial correlation in the function, thus indicating a bias towards good performance after good performance. They designed their tasks so that they could control the level of difficulty (error rate) for each. A Bernoulli process was employed to generate the expected performance rates at each error rate if responding were random. Gilden and Gray-Wilson chose a measure which they called the runs $z$ score to characterize departures from a Bernoulli process. $Z$ scores were calculated for subject performance, and were compared to expected values for numbers of streaks or runs of hits as expected under the Bernoulli process. T-tests were employed to compare subject $Z$ score values to expected values. Although there were no significant differences between actual and expected values at the easiest and most difficult levels of performance, a significant difference was revealed at the intermediate level of difficulty (hit rates 
ranging from .5 to .7 ) in both of their experiments (dart throwing and golf putting). This finding led the researchers to postulate that the fluctuations in skilled performance were washed out by tasks that are either significantly beneath the subject's skill level, or greatly above it. They conclude that there is some aspect of skilled performance that causes hits and misses to cluster together depending on task difficulty. Furthermore, Gilden and Gray-Wilson went on to examine four models which might describe the nonstationarity of hit rate in streaky performance. Of the models they examined, a wave modulation model, best described the observed sequence structure within the groups.

Though Gilden \& Gray-Wilson were able to identify and describe a wave-like structure to responding in skilled performance, they did not attribute this to attention. They describe a $U$-shaped function relating streak magnitude to hit rate, but had no means to relate hit rate to attention.

When analyzing the data in the present study, a methodology was chosen which loosely modeled Gilden and Gray-Wilson's general methodology in order to answer the primary question of the first experiment. That question was "Do hits occur at random times, or do they occur in runs?" By counting the number of hit runs and measuring the length of each run for both subject and simulated data sets, we attempted to determine if CPT hits tended to occur in streaks that are fewer and longer than expected by chance. The second question was also addressed, "If hits do occur in runs, is it because getting a hit causes a temporary increase in arousal that then raises the probability of detection of the following target, or more simply stated do hits beget hits?" Difference between subjects with good and poor performance on the CPT was closely examined using several dependent variables. 


\section{Methods}

\section{Participants}

\section{Part 1}

Each of the 40 subjects was given a rating based on their overall hit rate, i.e. percent correct detection of targets, as identified by the process described in the procedures section. Subjects were then grouped based on their rating. (See Table 1) The actual hit rate ranges for groups were determined subjectively after examination of subject data sorted by hit rate. A grouping method based on quartiles was discarded because it was not sensitive to the non-uniformly spaced subject hit rates. The mean group size was 10 subjects.

\section{Insert Table 1 Here}

\section{Part 2}

Two groups of subjects were formed from the original 40 based on their overall hit rate as described above. The first group contained the 15 subjects with the highest hit rates, and the second group, the 15 subjects with the lowest. (See Table 2)

Insert Table 2 Here

\section{Apparatus}

Please see the General Methods for a complete description of physical equipment used to collect and analyze these data sets. All specialized 
programming required for Monte Carlo data simulations was done by the author.

\section{Procedures}

\section{Transformation of Subject Data}

Starting with the data archived during CPT data collection, basic summary information was calculated for each subject. This was accomplished using a data extraction computer program (scoring program) designed and written by the author of this study (Appendix A). This program allowed flexibility in the criterion used to determine hits and misses for the data set. In this study, a hit was defined as a response occurring within a window of time starting 350 ms after the advent of the target stimulus, and extending out to $1300 \mathrm{~ms}$ after the advent of the target stimulus. Responses in less than $350 \mathrm{~ms}$ after the target stimulus were considered to be responses to the stimulus immediately previous to the target, and thus a false alarm (response to a non-target stimulus). Responses to non-targets occurring outside of the $1300 \mathrm{~ms}$ window after a target stimulus, or multiple responses to targets within the $1300 \mathrm{~ms}$ window were also considered false alarms. The outside value of the window which delineated a correct response to a target stimulus was referred to as the Maximum Acceptable Reaction Time (MART). A miss was defined as a lack of response to a target stimulus within the $1300 \mathrm{~ms}$ window. See Table 3 for a summary of subject grouping and performance. 


\section{Experiment 1 Part 1}

Descriptive statistics were computed for each of the four subject groups over multiple dependent measures.

Using medians to obtain the central number of misses for each group, four, one-thousand-case Monti-Carlo data sets were generated. The mean hit rate and number of misses used to create each Monti-Carlo group can be seen in Table 4.

Insert Table 4 Here

Monti-Carlo data sets were created by using a template of target times from the actual subject CPT, and assigning either a hit or a miss to each target based on a routine of random selection. The only non-random aspect of the resulting data set was the number of hits which was constrained to the hit rate of the group being simulated.

The simulated data sets were generated using several computer programs. Each simulated record contained 20 minutes worth of data. The master program controlled the creation and naming of each data set as it was generated. The following programs ran only once per data set. The first program determined where misses would occur in a temporal template of twenty minutes worth of CPT targets. The same stimulus template was used run all human subjects, and to create all 4000 simulated data sets, assuring that 
effects caused by target placement would be held constant. Placement of misses was accomplished by randomly selecting $X$ quasi-random numbers, between 1 and the maximum number of targets, using the computer's random number generator seeded with the time. $X$ was determined by the hit rate of the data set to be simulated, as noted in Table 4. No duplicate target numbers were allowed assuring that each simulated trial would have the same number of misses. The next program generated a simulation data file which contained target positions and hit/miss data. (See Appendix B for an example of a simulation data file.) This simulation file was interpreted by the scoring program as if it were an actual subject data file. Non-target data was filled in by the scoring program. Simulated data sets had no associated reaction times or false alarm rates. Once the simulated data had been created and scored, average number of hit runs, defined as hits on one or more consecutive targets, and average length of hit runs, defined as the number of targets included in each hit run, were calculated for each 1000 case group. These means were compared to human subject group means to using a one-way ANOVA. Due to the very large difference in group sizes, the alpha value was set a-priori to .001 . (See Appendix $\mathrm{C}$ for ANOVA tables.)

The average, maximum and minimum time between targets, was calculated using the stimulus template referred to above. The average , maximum and minimum times between targets for our stimulus template were $6.508,14$ and 2 seconds. We counted the number of hits and the number of misses following hits when the duration of the counted target from the previous target (the hit) was long (greater than or equal 6.508 seconds) or short (less than 6.508 seconds) for human subjects. The total number of opportunities for 
such events to take place was equal to the number of targets (185) minus 1 (the first target can not be counted). Using our stimulus template, we counted the number of short and long inter-target intervals and found them to be 110 short and 74 long. These numbers were used to create the expected expected value for the ratio, 1.49. Two-way Chi Square statistics were used to compare human subject group totals to the expected values. (See Appendix D for Chi Squares.)

Results, Experiment 1 Part 1

Insert Table 5 Here

Table 5 shows number and length of hit runs for human and simulated subjects. The results of the ANOVAs for Average Number of Hit Runs and Average Length of Hit Runs are as follows:

Group 1, poor performers

Average Number of Hit Runs $F(1,1010)=.713, \mathrm{p}>.001$ ns.

Average Length of Hit Run $F(1,1010)=13.366, \underline{p}<. .001$

Group 2, below average peformers

Average Number of Hit Runs $F(1,1009)=.003, p>.001$ ns.

Average Length of Hit Run $F(1,1009)=.254, p>.001$ ns.

Group 3, above average performers

Average Number of Hit Runs $F(1,1007)=.540, \mathrm{p}>.001$ ns.

Average Length of Hit Run $F(1,1007)=1.605, p>.001$ ns. 
Group 4, good performers

Average Number of Hit Runs $F(1,1006)=13.145, p<.001$

Average Length of Hit Run $F(1,1006)=81.875, p<.001$

Groups 1 and 4 show evidence of hit runs which are significantly different from those that would be expected at random. Longer hit run length and fewer hits runs are both indications that hits are occurring in runs rather than in a random pattern. Group 1 shows significantly longer hit run length, and group 4 shows both fewer hit runs, and longer hit run length.

A summary of the average number of Hits and Misses following Hits at Short and Long Intervals can be seen in Table 6. Table 6 also shows ratios short interval to long interval. Note that, for all groups, the ratios for hits following hits were all smaller than expected and misses following hits were all larger than expected, suggesting that there were fewer hits following other hits at short intervals and more misses following hits at short intervals.

\section{insert Table 6 Here}

Two-way Chi Square results for hits and misses following hits were for Group 1, Hits following Hits $X^{2}(1)=0.053, p>.05$, Misses following Hits $X^{2}(1)=0.145, p$ $>$.05; Group 2, Hits following Hits $X^{2}(1)=0.014, \underline{p}>.05$, Misses following Hits $X^{2}(1)=0.809, p>.05 ;$ Group 3, Hits following Hits $X^{2}(1)=0.025, p>.05$, Misses following Hits $\mathrm{X}^{2}(1)=0.560, \underline{p}>.05$; Group 4, Hits following Hits $\mathrm{X}^{2}(1)$ $=0.003, \underline{p}>.05$, Misses following Hits $X^{2}(1)=0.492, p>.05$. None were significant. 


\section{Experiment 1 Part 2}

Two groups of 15 subjects, good performers (the top 15) and poor performers (the bottom 15), were compared using a one-way ANOVA. Subjects were grouped according to their hit rate, and selected from our pool of 40 subjects. Groupings created in Part 1 were not used due to the their smafl n's. The groups were compared over several dependent variables. See Table 7 for group means for each variable compared, (See Appendix E for ANOVA tables.)

Results, Experiment 1 Part 2

Of the four variables compared, only Number of False Alarms $F(1,28)=$ $7.655, p<.01$, differed significantly between groups, with good performers having the significantly greater number. Average response latency as measured by Average Hit Time did not differ significantly between the two groups $F(1,28)=.709$, n.s. Ratios of Hits and Misses following hits at short and long inter-target intervals were not found to be significant between groups; Hits following Hits $F(1,28)=.5219$, n.s., Misses following Hits $F(1,28)=2.2102$, n.s.

\section{Discussion, Experiment 1 Parts 1 \& 2}

Experiment 1 asked "Do hits occur at random times, or do they occur in runs" as would be expected by a model of sustained attention that described attention as waxing and waning, having lapses separated by streaks of good performance. We went about answering that question by modeling trials in which hits do occur at random times, and comparing our human subject data to 
these trials on two measures, number of hit runs, and length of hit runs. Fewer hit runs or longer hit run lengths than were represented in the randomly distributed data sets would indicate periods of sustained responding separated by error runs. Evidence of this was seen for group 1, longer hit runs, and for group 4 as both longer and fewer hit runs. The results, while weakly supporting the position that hits occur in runs, were not representative of our entire group of human subjects. Any significant differences in either the length or number of hit runs from random models suggests that some non-random process within the subjects is at work, and that it is possible to differentiate human responding from random based on hit runs. These findings suggest that based on our CPT, this is possible in some cases. The fact that these differences were only found for the worst and the best performers is interesting, and may become important as we progress in our investigation, as it may provide a clue to the CPT's sensitivity for detecting fluctuations in average performing individuals. Gilden \& GrayWilson, as noted earlier, identified differences between human responding and a Bernoulli model using these same variables, but noted that tasks which were too easy or too hard washed out this effect. Although these results seem to suggest that average responder's effects are masked, that it may be a characteristic of the task itself makes it difficult to identify non-random performance. The finding also lead us to a related question, "If hits do occur in runs (and we have noted that they do in some situations), is it because getting a hit causes a temporary increase in arousal that then raises the probability of detection of the following target", or more simply stated do hits beget hits? This question is important because it addresses a possible mechanism to explain fluctuations in attention as the cause of the hit runs which we have identified. 
The non-significant results for any group on the Chi Squares looking at hits following hits indicates that there is no evidence of increased likelihood of hits occurring after hits at short intervals as would have been expected if an arousal process was at work. In fact, based on the non-significant results on each of the Chi Squares in Experiment 1 Part 1, there was no evidence of any effect based on making a hit. In every group, however, hits were more prevalent than expected after a long interval and less prevalent after a short interval. Misses were more likely to occur than would be expected after a short interval, and less likely after a long interval. The non-significant results of the Chi Square tests of misses following hits suggests that there is no organizing factor for hit responses due to expectation, but there still seem to be hints of expectation effects after longer intervals without a target. Further investigation into these effects is warranted.

The primary objective of Part 2 of Experiment 1 was to compare data summaries of accuracy data in order to determine if there were any features to the data which would allow simple differentiation between good and poor performers. Identification of such features would be extremely helpful in the development of tools to identify individuals with attentional difficulties. There were only two intriguing findings. The first was that good performers made significantly more False Alarms than did the poor performers. This could indicate a real difference in the strategies used by individuals when performing a task like this one, or perhaps a simple difference in motivation. The second finding was that there was no significant difference in the response latency between good and poor performers. This is interesting because it invalidates the idea that accuracy might be based on time taken to process stimulus data, 
an appealing alternate hypothesis to vigilance in the variation in responding seen over time.

At the end of Experiment 1, some questions had been answered, but additional questions remained. Some support was found for the existence of non-random hit run mechanism in human performance which is as of yet unexplained, but is not due to relationships between hits, and a procedure that seems capable of identifying non-random hit runs in CPT data. As a next step in the investigation, it seemed reasonable to examine another aspect of hit runs. The existance of runs had been demonstrated, but there was no information about when they occur, or if they might be in regular or random patterns. These questions were examined in Experiment 2. 


\section{Experiment 2}

Wave Forms in Attention as Measured by Momentary Hit Rate in CPT Performance

Experiment 1 examined the nature of clusters of hits or runs in responses of both human and modeled case data. The object was to test a procedure for quantification of fluctuation in attending over the course of a CPT, i.e. determine whether there were fewer but longer runs of hits in human subject data than would be expected by chance. Also examined was the relationship between successive hits and hit-miss sequences to determine whether or not making a correct response, a hit, influenced the probability of making a correct response on subsequent targets. However, these examinations do nothing to examine the possibility that runs of hits have a periodic nature, i.e. that they occur at one or more regular intervals. A subject could have a significantly low number of runs, but the runs may or may not be periodically distributed. A subject could also have a number of runs not significantly different than what would be expected by chance, but the runs could show periodicity which would not be expected to happen by chance. What methods could be applied to CPT accuracy data to detect periodicity? If there is periodicity, does it differ from that which might be seen by chance, and is it due to some property of the test (e.g. most obviously, the targets are periodic) or to some property of human attention? These questions are the basis of Experiment 2. Experiment 2 utilized spectral analysis tools to reveal periodicity in accuracy performance. A Fast Fourier Transformation (FFT) analysis of momentary hit rate functions was used to create a power spectrum of hit run frequency for each subject. This power spectrum was used to determine if there was a periodicity to correct target 
detections, and if that periodicity differed from any that might be seen in cases modeled randomly (described in Experiment 1 Part 1). A result showing periodicity in human subjects, but not in modeled cases would indicate that some process intrinsic to the performer, assumedly attention oscillations, were regulating human performance. In order to apply the FFT to CPT accuracy or reaction time data, however, the data had to be transformed into a digital function with a standard (and known) time base. This was accomplished by creating Momentary Hit Rate and Momentary Reaction Time functions.

Part 1 of Experiment 2 examined our procedures for creating a Momentary Hit Rate/Reaction Time function, a quantification of moment to moment changes in accuracy/response latency over the course of a trial. Seven data sets with programmed (embedded) frequencies were created and run through a Moving Average Window data processor which created momentary functions. This computer program converted binary accuracy data into a continuous performance based function and standardized the time base.

In Part 2 of Experiment 2, the seven resulting MAW functions, along with four functions created specifically for FFT analysis, were submitted to a Fast Fournier Transformation process, the purpose of which was to create power spectra which would reveal periodic wave forms in the data.

Part 3 of Experiment 2 examined each of the 40 subjects individually. Based on the results of the FFT procedure run on each subject, the resulting frequency information was arranged into categories. Next, 40 randomly selected modeled data sets from the 4000 created in Experiment 1 were submitted to the same FFT procedure. A Two-way Chi Square was performed 
between the human subjects and modeled data for the frequency band which was identified from the FFT results.

Experiment 2 Part 4 compared FFT findings from good and poor human performers as identified in Experiment 1, Part 1. Two-way Chi Square statistics were used to compare group membership in different frequency bands (bins). Significant differences in group membership would suggest differing periodicities between varying levels of responder based on response accuracy.

\section{Methods}

\section{Participants}

Please see the General Methods for a complete description of study participants. Eleven simulated data sets were created, 4 for FFT analysis only, and 7 for momentary hit rate and FFT analysis. Forty simulated data sets from Experiment 1, with errors randomly distributed, were selected randomly, 10 from each Monti-Carlo group.

\section{Apparatus}

Please see the General Methods for a complete description of physical equipment used to collect and analyze these data sets. All specialized programming required for FFT analyses was done by the primary author of this study.

\section{Procedure}

Experiment 2 Part 1: Preparing the Data for FFT analysis: Creating Momentary Hit Rate Functions

The binary nature of raw accuracy data leaves the researcher with few options. Counts, averages and ratios are as complex as most CPT analyses get. In order to apply some more powerful methods for describing fluctuations 
in responding to our CPT data, the data must be put into a form accessible to an FFT or other spectral tool. At the same time, however, it must be transformed in a way that will not distort underlying periodicity in the data, or introduce artificial periodicity to the function. To that end, the author developed a Moving Average Window (MAW) procedure.

The MAW is a smoothing technique employed for various tasks and excellent for producing a function based on raw CPT data. It provides a more revealing visual representation of the data. It also serves a second more important purpose in this case, which is to take a function with a variable time base, such as the CPT accuracy function, and create a new function from it which has a standard time base. The MAW procedure functioned in the following manner. A set of raw CPT data, containing information about both target and non-target stimuli was selected. It was important for stimuli themselves to have a standard time base. The CPT presented stimuli at 2 per second. Targets occurred at various intervals, from 2 to 14 seconds. A window, 30 seconds in length, was placed over the raw data function. Thirty seconds was chosen because the average time between targets was 6.5 seconds, and this provided 3 to $7(\underline{M}=4.6)$ targets within the window at any given time. This window was then stepped forward on the raw data function in 6 second increments. With each step, an average of subject performance within the window was created. Performance was based on hits (correct responses to target stimuli) as described in Experiment 1 Part 1. Hits were given a value of 1 and misses a value of zero. Thus, if there were 4 targets within the window, and 3 of those targets were hits, a score of .75 would be recorded for that window. With each step it was typical to drop 1 target from the back of the window and 
encompass 1 new target in the front of the window. The window was progressed along the raw data function until it encompassed the last stimulus. This process resulted in a digital function with 185 evenly spaced $(6 \mathrm{sec}$ ) data points (window averages) ranging from 0 to 1 . The whole process was automated, and contained in the Scoring Program written by the author of this document. (See Appendix A for a listing of the Scoring Program) The data points of the new function themselves are a quantification of accuracy performance at each time point in the CPT. In a preliminary pilot study, visual analysis of functions before and after MAW processing indicated that the MAW process did not distort the actual performance functions. Our MAW process was used with all data for Experiments 2 and 3 unless otherwise noted.

In order to test the accuracy and reliability of the MAW process, and to verify that the process itself neither distorts nor adds periodicity in the data, test data was created which could be processed by the MAW, and later interpreted by the FFT. Seven cases were created, the first four of which were programmed with a single period of oscillation and additional noise data varying from none to high. The last three cases were programmed with two periods each. To create these cases, several steps were required. The first involved using a spreadsheet program with a set of target stimulus locations extracted from the CPT stimulus template. Having determined the stimulus number, we then calculated the actual minute in which the stimulus was presented, 0-19. Next, we assigned a hit or a miss to each target based on the frequency of the period we were creating, and the time of each target. Various levels of noise (random-error having nothing to do with sound) were added by spreading out hits around the actual position of the targets required to create 
the chosen periodicity. All this information was saved, and submitted to a second program, developed by the author, which created a simulation file which could be read and interpreted by the MAW procedure. (See Appendix F for a listing of this program.) This file contained only target information. Non-target stimulus information required to complete analyses was automatically added by the MAW procedure. MAW processed case data are examined with the FFT in Experiment 2,Part 2.

Results, Experiment 2 Part 1: Creating Momentary Hit Rate Functions See Figure 1 for the distribution of the number of targets falling within the MAW.

Insert Figure 1 Here

See Table 8 for MAW test case summary information.

Insert Table 8 Here

For the purposes of illustrating the procedure, graphical representations of the MAW functions for cases 1 and 4 can be seen in Figures $2 \& 3$.

Insert Figure 2 Here 
Insert Figure 3 Here

The MAWs, which represented digital functions of performance over time with a fixed time interval between data points, had an interesting feature in these test cases. They not only showed regular oscillations, which were programmed, but also looked like a pictorial record of attention lapses if viewed upside down, and read right to left. These representations could give a nicer impression of performance over time than is provided by a table that shows percent correct in each two minute block, which is the conventional way of looking at performance over time.

Experiment 2 Part 2: Spectral Analysis \& the FFT Procedure

\section{Introduction to spectral analysis}

Spectral analysis is a powerful set of procedures which can be used to identify periodic wave forms in linear data sets. There are, however, certain requirements which must be met if the procedures are to operate properly. One of the most fundamental is the requirement of a fixed time base. Without the fixed time base, it is not possible to interpret frequencies within a function. Due to the fact that human beings are capable of quickly learning to anticipate targets if they occur at regular intervals, targets in the CPT were programmed to occur at variable intervals. A procedure was required which could convert variable interval target data into fixed base data. The MAW process accomplished this, allowing us the unpredictability of a variable interval 
schedule for target presentation, while providing a powerful set of analysis tools to search for and quantify periodicity in the CPT data.

The FFT was selected as the spectral analysis operation. The FFT operation was programmed within the MatLab software package. (See Appendix $G$ for the program listing) It was chosen primarily due to the fact that it had been tried in the literature, and proved capable of analyzing data similar to that of the CPT used in this study (See Makeig \& Conte et al.).

The FFT itself is a complex identification process. Using mathematical operations, the process produces measures which indicate the degree to which frequencies of a broad range are detected in the data function. These measures are most interpretable when represented graphically in a power spectrum. The power spectrum is a chart with a range of frequencies on the category axis, and a measure of power (the degree to which frequencies of a broad range are detected) on the value axis. The data series on the chart maps the existence of frequencies in the tested function.

Within the FFT procedure, some mechanism for identifying primary frequency spikes from noise spikes in the FFT power spectrum was required. To accomplish this task a critical value was created that was termed the Least Significant Power (LSP), and which identified the power level at which spikes were determined to be primary frequencies. All spikes below the LSP in any given power spectrum were ignored. The default LSP was set to 20. This was a good number for cases which had a maximum observed power of 100 or less. This setting caused the procedure to ignore the majority of the sampling noise, and identify only strong primary frequencies. When the maximum observed 
power was very low, i.e. $15-25$, the program automatically adjusted the critical level to $1 / 2$ the maximum observed power.

In preliminary testing of the FFT program during its development stage, the use of a data filter called a "Hamming Window" greatly reduced the level of extraneous legs or noise spikes surrounding primary frequencies. After testing, to make sure the Hamming Window did not suppress primary frequencies, it was adopted as a precursor procedure to the FFT in all cases. Use of the Hamming Window greatly assisted in the identification of primary frequencies.

Due to the complex nature of the FFT algorithms and the complicated nature of their output, the authors set out to test the procedures to be used in the remaineder of this study in order to determine if they were both reliably measuring periodicity in the processed data and were valid for determining the nature of the periodicity. This was accomplished in the following fashion. First, the FFT alone was tested using simulated data sets, created in the following fashion. Target periodicity and function time base were chosen, and period data was entered into a spread sheet program. In some cases, noise was added to the function to see if this would obscure the frequency to the FFT procedure. Noise was added by randomly choosing a percentage of the targets and reversing their values, hits became misses etc. This data was saved and was ready for processing by the FFT process.

Four test cases were created. Each had a 6 second time base. Case 1 was programmed with a 5 minute periodicity and no noise. Case 2 was programmed with a 5 minute periodicity and $20 \%$ noise. Case 3 was programmed with a 5 minute periodicity, and $40 \%$ noise. In case 4 , two frequencies, 1 minute and 5 minutes, were programmed into the function with 
no noise. This test was designed to verify that the FFT was capable of picking two separate but overlapping frequencies. Each case was submitted to the FFT procedure and the resulting power spectrum was assessed for accuracy .

To complete the examination of the procedures, testing of the MAW/FFT procedural chain, a pair of procedures that were instrumental in the analysis of the CPT data. MAW test cases were created in Part 1, with known periodicities. These cases were submitted to the MAW, and fixed time base Momentary Hit Rate functions resulted. These functions were submitted to the FFT procedure for analysis, and the resulting power spectrum was assessed for accuracy. (See Appendix $\mathrm{H}$ for some good examples of clear periodicity visible in MAW and FFT.)

Results, Experiment 2 Part 2: Spectral Analysis \& the FFT Procedure For FFT only test cases, the FFT procedure was able to identify the periodicity of the programmed frequency accurately in 4 out of 4 cases. In case 1, (See Figure 4) the FFT procedure resulted in a period of 5.115 minutes.

\section{Insert Figure 4 Here}

In case 2, the FFT procedure result was 5.115 minutes (See Figure 5) which was again very close to the programmed frequency.

Insert Figure 5 Here

Note the additional low power peaks in the higher frequencies of Figure 5. These are the direct result of the masking noise added to the function. 
The test function for Case 3 was created in precisely the same fashion as in Case 2 with the exception of the amount of masking noise added. The Case 3 function contained approximately $40 \%$ masking noise, leaving only slightly more than half the function intact.

Insert Figure 6 Here

Despite this, the FFT result showed a period of 4.87 minutes. (See Figure 6) Note that the power of the primary frequency has been severely reduced. The direction of the error in the result has also shifted from a longer period than the one actually programmed to a smaller period, although the error is still small.

Insert Figure 7 Here

In Case 4, two frequencies, were programmed. (See Figure 7) Both frequencies were readily identified in the power spectrum resulting from the FFT procedure.

The results of the MAW/FFT test cases follow. The power spectrum resulting from the FFT of the first Case is shown in Figure 8 . Note the clear 2 minute period, and the low noise. The second visible spike is at precisely $1 / 2$ the frequency of the primary spike, and is called a harmonic. Harmonic frequencies should not be interpreted. 
Insert Figure 8 Here

Note that the FFT identified a period of 2.006 minutes, extremely close what was programmed. In cases 2,3 and 4, noise was gradually added to the 2 minute period. In each Case, low, moderate and high noise, the FFT procedure accurately identified the 2 minute period, but changes in the noise effects were noted. Figure 9 shows the power spectrum for low noise.

Insert Figure 9 Here

Note that the noise level is very low except for a single extraneous harmonic spike which actually occurs at 1.003 minutes. This same spike appears in the Case 3 spectrum, but the power of the harmonic spike has increased significantly and the overall power of the fundamental frequency has been reduced. (See Figure 10)

Insert Figure 10 Here

In Case 4, we increase the noise even further, but the result is not the further degradation of the signal (See Figure 11), but rather the splitting of the noise into several distinct frequencies and the noticeable luss of the harmonic. 
Insert Figure 11 Here

The final three tests run on the MAW/FFT procedure chain were dual frequency trials.

Insert Figure 12 Here

In each Case, the combination MAW/FFT procedure was able to identify the programmed periods (See Figures $12,13 \& 14$ ), however, it was noted that as the binary data of which these functions are created overlapped more, extraneous spikes were created.

\section{Insert Figure 13 Here}

This is especially visible in Case 6 (Figure 13), where a 1.329 minute period has been detected and only $1 \& 5$ minute periods were programmed. In Case 5 (Figure 12), periods of 3.935 and 2.006 were identified by the FFT, close to 2 and 4 programmed. The results for Case 6 were $4.092,1.329$ and 1.003. The extraneous spike is indistinguishable from the expected periodicities in terms of power. In Case 7, the FFT identified periods of 5.115 and 1.003. (Figure 14) Although there wc.. several subspikes visible, none were comparable in power to the primary frequencies. 


\section{Discussion, Experiment 2 Parts $1 \& 2$}

The results of the testing of the MAW and FFT procedures were most encouraging. With the exception of minor variation in the detected frequency from the programmed frequency, all detections were extremely accurate. The effect of noise on the procedure was two-fold. It produced extraneous spikes in the power spectrum and reduced the overall power of the primary periodicities. This result was not unexpected, however. As noise in functions increases the number of possible solutions to which frequencies exist in the function are bound to increase. At the same time, the primary frequencies are 'blurred' and harder to differentiate, which serves to lower their power. In MAW functions the overall level of noise power in the FFT power spectrum seemed to be suppressed over that of the non-MAW test functions. MAW functions instead showed some harmonic power, but this did not appear to be a significant problem in interpreting the results. In each case the highest spike related to the harmonic was the primary frequency. Some important facts about the nature of our binary data were revealed as well. Due to natural masking, it is not possible to detect frequencies which are close together. At times it seemed that extraneous spikes or artifacts are raised in the power spectrum, and that these spikes were difficult to distinguish from actual primary frequencies.

Overall, however, the tests of FFT and MAW/FFT both yielded excellent results. The FFT procedure was sufficiently robust to detect programmed 
frequencies even when relatively high levels of noise were introduced. The MAW procedure showed no signs of having distorted the underlying frequencies programmed into the data, and even seemed to act as a noise suppression tool.

\section{Experiment 2 Part 3}

\section{Procedure}

Momentary hit rate data for each of our forty human subjects was run through the MAW/FFT procedure chain as described in Parts $1 \& 2$ of Experiment 2. Identified periodicities were recorded for each subject. When running our FFT function, it was necessary, with some cases, to adjust the LSP to correct for unusually high power spikes, or higher than normal noise levels. This adjustment served to accentuate primary frequencies.

Once all of the human subject FFT data had been recorded, identified periods were categoriezed. Subjects showed as few as 1 and as many as 6 identified periodicities. Upon visual analysis of the data, it appeared that there were natural concentrations of frequencies centered at approximately $1,2,5$ and 15 minutes. Bins were created to categorize data. Each bin spanned a range of possible frequencies, being centered on one of the natural concentrations mentioned above, but as a group, all possible frequencies were covered. Bins were created subjectively to encompass all data while remaining as sensitive as possible to subjective natural bands in the subject data. The four bins used initially to categorize subject data ranged 0-1.5 minutes, 1.51-3.5 minutes, 3.51-8.5 minutes and 8.51 and more minutes respectively. Quantatative analysis of primary subject frequencies was conducted to validate 
the existance of bands within subject data. Only subjects with best power (i.e. power in their power spectrum of 20 or greater) were included in this analysis reducing the group size to $16(\underline{n}=16)$. The top 4 identified frequencies were recorded and examined. (See Table 9 for a summary of the subjects and frequencies.)

Insert Table 9 Here

Of the 16 subjects 12 had a major wave band at between 4.7 to 6.0 minute cycles. 15 of the 16 subjects show a fast wave band of between 1-2 minute cycles. Many of the subjects have two frequencies in this range, suggesting the possibility that there may be two separate bands included in this range. Finally, 4 of the 16 subjects showed a slow wave band at 10 minute cycles or greater. Most of the peaks identified by the FFT are encompassed within these three wave bands. There are extraneous peaks between 2.8 and 4.1 minutes, and seeming somewhat focused around 3.1-3.2 minutes, which could represent a fourth wave band, but there are relatively few. The narrowed width and wide separation of the observed wave bands for subjects with best power lent credence to the process used to categorize all 40 subjects. Although there was more variability in the identified bands for lower power FFTs, the process of categorization appears to have been viable, and the results comparible, especially after bins were narrowed (see below).

Periods for the 40 subjects were assigned to their respective bins, and descriptive statistics were calculated for each bin. Based on the observed mean for each bin, a new narrowed frequency range was assigned. It was 
equal to the bin mean \pm 1.5 times the bin $\underline{\mathrm{SD}}$. Limiting the bin ranges in this way allowed us to eliminate some group outliers, and in most cases to increase distance between categories. Bin membership was reassessed.

Next, forty simulated data sets with errors randomly distributed were selected randomly, 10 from each Monti-Carlo group created in Experiment 1. Each simulated data set was run through our MAW/FFT procedure chain as each human subject had been. The resulting periodicity information was recorded. Each recorded period which fell into a bin range was then assigned to one of the narrowed frequency range bins. Human and simulated data were held separate at all times. Once all bins were finalized, the maximum and minimum scores were taken for each, and these became the actual bin ranges. They represent the actual range of frequencies for FFT data within the bin.

Descriptive statistics and percentages of subjects falling into each bin were then calculated for human and simulated subjects. A two-way Chi Square statistic was generated for each bin between human and simulated subjects in order to identify if human frequency membership in each bin differed from that expected by chance. (See Appendix I for Chi Squares.)

\section{Results, Experiment 2 Part 3}

The results of the original categorization of subjects into bins can be seen in Table 10, which shows the percent of subjects with identified spikes falling within the bin.

Insert Table 10 Here 
A summary of narrowed bin ranges for human subjects and of actual ranges for both human and simulated subjects can be seen in Table 11.

Insert Table 11 Here

Table 12 shows the percentages of human subjects and simulated subjects who had frequencies which fell into each narrowed bin. These are the values which were used to calculate the Chi Square statistics. Note that human subjects have consistently higher bin membership.

Insert Table 12 Here

Two-way Chi Square analyses were conducted for the percentage of subjects, human vs. non-human, with a period in each bin. For bin $1, X^{2}(1)=$ $0.457, \underline{p}>.05 ; \operatorname{Bin} 2 X^{2}(1)=2.739, p>.05 ; \operatorname{Bin} 3 X^{2}(1)=1.270, p>.05 ; \operatorname{Bin} 4$ $X^{2}(1)=3.660, \underline{p}>.05$. No significant differences between human and modeled accuracy periodicity was found for any of our bins.

\section{Discussion, Experiment 2 Part 3}

Part 3 examined several aspects of subject periodicity. Based on the findings in Experiment 2 Part 2, it is necessary to assume that the FFT procedure accurately described periodicities existing in the CPT and modeled momentary hit rate functions. Although periodicities were revealed, statistical analysis could not differentiate these from random. One possible explanation of this result was there is no subject related periodicity, and that the periodicity 
observed was related to some property of the test structure. The fact that the percentages of human subjects within each of the longer period categories, 2,3 \& 4, were higher than with random subjects, however, suggests that perhaps the statistical procedure used was not sensitive enough to discriminate the differences, or perhaps that there was simply too much noise mixed in with the lower power subjects. The process for determining which periods were primary, and which resulted from sampling noise was difficult at best. The narrow bands and high numbers observed when only the best power subjects were examined bolster this idea. It was also recognized that given a small number of errors, such as what was obtained by human subjects, and modeled in the simulations, the actual range of possible periodicities is narrowed, making it more likely that random and non-random responding would appear similar. More investigation is required before the validity of the this method for differentiating human and random periodicity can be determined.

Experiment 2 Part 4: FFT comparison of Good and Poor Human Accuracy Responders

Categorized FFT data for human subjects from Expeiment 2 Part 2 was broken down by subject groups as defined in Experiment 1, Part 1. The number of subject group members appearing in each bin was analyzed with a two-way Chi Square (see Appendix $J$ for Chi Squares). Percentage membership within bins is listed by group in Table 13. 
Results, Experiment 2 Part 4: FFT comparison of Good and Poor Human Accuracy Responders

Results of the Chi Square test run between subject accuracy level groups for bins $1,2,3 \& 4$ were not significant. For bin $1, X^{2}(3)=1.261, p>.05$; Bin 2 $X^{2}(3)=0.475, p>.05 ; \operatorname{Bin} 3 X^{2}(3)=0.095, p>.05 ; \operatorname{Bin} 4 X^{2}(3)=0.276, p>.05$.

Discussion, Experiment 2 Part 4: FFT comparison of Good and Poor Human Accuracy Responders

The primary purpose of Part 4 was to check for possible differences in periodicity between subjects of different accuracy levels. Considering the results in Expeiment 2 Part 3, it is not surprising that there were no significant differences between subject groups. The fact that subjects from different hit rate groupings did not differ in response periodicity verifies that accuracy is not related to periodicity of responding. In this case, it is safe to say that for the whole range of human performers on our $\mathrm{CPT}$, good and poor performers showed no difference in periodicity of accuracy response.

\section{General Discussion for Experiment 2}

Experiment 2, sought to provide understanding of the nature of response periodicity in human responding on a CPT. The goals were to quantify moment to moment fluctuation in responding, to identify proper procedures and tools for identifying and measuring periodicity in responding, to assess the differences 
between human and random response frequencies, and to compare good and poor human responders in terms of their underlying response periodicities.

One of the greatest accomplishments of Experiment 2 was the development and testing of the tools and procedure necessary to quantify and measure fluctuations in CPT responding. The Momentary Error Rate function created by the MAW procedure accurately tracked the level of response accuracy over the course of the CPT. It effectively converted a binary function into a non-binary function, and even provided a method of standardizing the time base of the function so that it could be analyzed effectively by spectral analysis tools. It was also readily interpretable when represented graphically. The FFT is an excellent tool for identifying underlying frequencies in functions, and has proved useful in a wide variety of applications from electrical engineering to medicine. The fact that the FFT was unable to provide a resolution to our search for a tool for descriminating human accuracy response patterns from random patterns does not diminish the fact that it performed admirably in Part 2. The fact is that sampling noise may well have been overwhelming in the data, and that our procedure for identifying noise was ineffective.

In addition, while the statistical analyses did not support the conclusion that any periodicity that was detected was the result of some property of the performer, such as attention, qualitative examination of the data seems to suggest that additivi. i research is warranted. One possible explanation of the result was that periodicity was the result of the measurement or the CPT structure, i.e. the distribution of targets in the stimulus template or that higher hit rates naturally have fewer errors and thus produce longer frequencies in 
accuracy data. An alternate explanation was that due to the fact that the simulated data were generated by simply shuffling the locations of errors, that the procedure was simply not sensitive enough to distinguish performer based oscillation from random fluctuation when looking only at binary accuracy. These results do not necessarily rule out performer driven results in accuracy responding. As noted earlier, Makeig \& Inlow (1991) reported non-cyclical fluctuations in responding starting at 5 minute lengths which would not have been picked up by our FFT, but could have been evident in Experiment 1. Conte et. al. (1995), suggested that there are periodic frequencies which are at least 4 minutes in length, and which don't seem to be standard from person to person. While periodic oscillations are evident in the results, the current procedures were unable to differentiate them from random simulations.

Human attentional mechanisms are extremely complicated, and no model as yet postulated suggests that there are specific and standard timers to control the vigilance system. It certainly is an intriguing possibility however. Despite the lack of evidence for unique human response patterns in the results of this experiment, we did succeed in quantifying and measure fluctuations in CPT data effectively. It may be important to note that response accuracy periodicity seemed to remain standard between subjects from differing accuracy levels, although one must take the findings of Experiment 2 Part 2 into account when considering this.

How many of problems were related to data collection, and the binary nature of accuracy data? That is a question that is difficult to answer, but Experiment 3 again examines response periodicity, this time using a linear dependent measure, reaction time. 


\section{Experiment 3}

Wave Forms in Attention as Measured by Momentary Response Latency in CPT Performance

While accuracy, by its very nature, seems the most obvious indicator of attention, the findings in Experiment 2 have led us to consider the idea that if fluctuations in accuracy responding are due to some property of the CPT structure, then perhaps fluctuations in attention may be more clearly identified by the general lengthening or and shortening of response latency (reaction time). Experiment 3 , like Experiment 2 before it, was designed to examine the possibility that there are identifiable periodicities to human responding. Due to the non-binary nature of the reaction time measure, which made it less susceptible to ceiling and floor effects, it was postulated that reaction time (RT) might prove to be a more sensitive measure of fluctuations in attention than the more traditional accuracy measure as well as being more robust against patterns imposed by the CPT itself. To our knowledge, this is the first study to use RT in this fashion.

Part 1 of Experiment 3, assessed human subject RT data for periodicity using the MAW/FFT procedure described in Experiment 2. In many ways, Experiment 3 was similar to Part 2 of Experiment 2. Simulated data was not available for comparative analysis, however, due to the fact that the simulations did not contain RT information. Thus, no comparative analysis of RT periodicity to random periodicity was possible. FFT results for RT were examined and bins were chosen by the same subjective process followed in Experiment 2. Similarities and differences in the resulting bin membership and mean 
periodicities are discussed. Bin membership between accuracy and reaction time were compared using a Two-way Chi Square statistic.

In Experiment 3 Part 2, we visually compared FFT power spectra from RT and accuracy for each subject. Similarities and differences are noted and discussed.

In Experiment 3 Part 3, we compared group FFT findings for good and poor human performers as identified in Experiment 1 Part 1. Two-way Chi Square statistics were used to compare group membership in different periodicity ranges (bins). Significant differences in group membership would suggest differing periodicities for RT between varying levels of responder based on response accuracy.

\section{Methods}

\section{Experiment 3 Part 1}

\section{Procedure}

Raw RT data for forty human subjects was submitted to the MAW process. The resulting function was a Momentary Reaction Time (MRT) function which serves to estimate the probable reaction time at any moment during the CPT. Please refer to Experiment 2, Part 1 for an in-depth description of the MAW procedure. The only difference between Momentary Hit Rate and Momentary Reaction Time is the measure upon which it is based. Each newly created MRT function was then submitted to the FFT procedure. Due to the large range of the RT data, it was necessary to create a residual function from the raw data to create an interpretable result. This was accomplished by subtracting the mean of the entire raw function from each point. Preliminary testing indicated that this procedure did not mask embedded periodicity in the 
function. Periodicity data resulting from the FFTs was recorded. Please refer to Experiment 2, Part 2 for additional information regarding the FFT operation. It was noted that RT data generated between 1 and 8 periods per subject with the mean number around 5.

RT periods were assessed visually, in order to identify natural bands of response frequency. Following the procedure stated in Experiment 2, Part 3, 4 bins were chosen. It was noted immediately upon inspection of the data that the frequencies identified for RT were very comparable to those identified for accuracy in experiment 2. After careful consideration. it was decided that the bins would have the same ranges as the original frequency range categories identified in Experiment 2, Part 3.

As it was in Experiment 2 Part 3, quantatative analysis of primary subject frequencies was conducted to validate the existance of bands within subject RT data. Only subjects with best power (i.e. power in their power spectrum of 20 or greater) were included in this analysis reducing the group size to $23(\underline{n}=23)$. The top 4 identified frequencies were recorded and examined.

RT bin information was compared to accuracy information using two-way Chi Square statistics in order to determine if bin membership was significan ly different between modalities. (See Appendix K for Chi Squares.)

Results, Experiment 3 Part 1

Table 14 shows a summary of identified RT frequencies for best power subjects. 
13 of the 23 subjects with best power evidenced a major wave band at between 4.7 to 6.0 minute cycles. 18 of the 23 subjects showed a fast wave band of between 1-2 minute cycles. Many of the subjects have two RT frequencies within this range, as well, suggesting the possibility again that there could be two wave bands represented within that range. 8 of the 23 subjects showed a slow wave band at 10 minute cycles or greater, double that of accuracy. Again, most of the peaks identified by the FFT were encompassed within the three wave bands. There were still extraneous peaks, which could represent a fourth wave band, but there was no strong evidence for this. The observation of bands within the best subject data, and the fact that those bands were relatively close to chosen bands, once again supported the categorization procedure used for the full subject complement. RT periods were sorted into each bin, and descriptive information was calculated. Then the bin range was narrowed according to the procedure in Experiment 2, Part 3. Descriptive information was recorded and differences between RT and accuracy data were subjectively assessed.

Insert Table 15 Here

Table 15 shows a comparison of bin ranges between subjects on the RT measure and the accuracy measure. Note that in almost every case, the 
difference in ranges are small, 20 seconds or less. The exception to this is Bin 4 which differs by over 10 minutes.

Insert Table 16 Here

Table 16 shows the means, SD's and percentages of RT data and Accuracy Data which had frequencies which fell into each narrowed bin. Mean periodicity, SD of periodicity and Membership between RT and accuracy are very comparable except in bin 4 . Mean periodicity is typically different by 20 or fewer seconds and bin membership is within 3 subjects for bins 1,2 \& 3 .

The results of the two-way Chi Square analyses were, for Bin $1, X^{2}(1)=$ $0.061, \underline{p}>.05 ; \operatorname{Bin} 2 X^{2}(1)=1.867, \underline{p}>.05 ; \operatorname{Bin} 3 X^{2}(1)=0, \underline{p}>.05 ; \operatorname{Bin} 4 X^{2}(1)$ $=6.146, \underline{p}<.05$. Only bin 4 showed significant difference with 23 of 40 subjects showing up for RT and only 12 for accuracy.

Discussion, Experiment 3 Part 1

Comparisons of features from accuracy and RT FFT data for our 40 human subjects turned out to be remarkably similar. This was not terribly promising considering the fact that periodicities found in Experiment 2, Part 3 were could not be related to traits of subject's responding. It wasn't until bin 4, the long frequencies, was reached that there seemed to be a difference between periodicity in accuracy and reaction time. It was quite evident from all measures that there were differences there. These differences were confirmed by the chi-square. Bin 4, the long periods, ranged greatly for RT. The fact that the same Chi Square analysis between accuracy for human and simulated 
subjects was not significant for Bin 4, but did approach significant levels might indicate that there is an as of yet unidentified phenomenon involving long response periods buried deep in the data. The fact that only 20 minutes worth of data was collected makes it difficult to investigate this phenomenon further. The result of the Chi Square may also indicate that, considering the similarity of the other 3 categories, the RT data is more sensitive to longer frequencies.

What is the significance of this difference? That is more difficult to determine. Without a random measure of reaction time periodicity, it is still impossible to claim that the periodicity in bin 4 is non-random. Thus at this time, the only claim that can be made is that reaction time and accuracy periodicity as measured by our MAW/FFT procedure differ significantly in a frequency range of 8.5 to 34 minutes with RT FFT results showing significantly more frequencies within that range than accuracy.

\section{Experiment 3 Part 2}

\section{Procedure}

The power spectra from RT and accuracy FFT results matched by subject were examined visually. Power spectra were overlaid, allowing a point by point comparison of the data function. Due to the fact that the category axis is the same in all of the cases, frequencies were comparable. Notes on observed traits of subjects grouped by hit rate as they were in Experiment 2 Part 4 were included.

RT and accuracy spectra were examined on a few criteria. The first was primary frequency power. This was a relative comparison of power of the most dominant frequencies. Differences in the general power of noise to the power 
of the primary frequency bands were compared to assess the clarity of the band against the noise in the function. The ratings were clear (the spikes stand well above the rest of the spectrum), moderately clear (The spikes intermingle with noise in some locations), and not clear (spikes are not well distinguishable from the rest of the function).

The second was overall noise level. This was a subjective measure of the overall level of noise visible in the function, assessed as high, moderate and low.

The last was comparability of primary frequencies. This was an assessment of the similarity of RT and accuracy primary frequency bands. Locations of the primary frequency bands were compared between the two spectra, and rated as same (obvious frequency bands in same locations, few deviations in frequency), similar (obvious frequency bands, slightly offset or raised in different locations), or different (spikes don't fall in the same bands). Power was generally disregarded in preference to frequency band.

The number of subjects with RT and accuracy FFT results falling into each category for each variable was totaled, and percentages were calculated. See Appendix L for subject accuracy and RT spectra.

Results \& Discussion, Experiment 3 Part 2 A comparison of the primary frequency power can be see in Table 17. 


\section{Insert Table 17 Here}

The amount of power found in the primary spikes relative to the rest of the function was very comparable for accuracy and reaction time. Both groups had the same percentage of clearly differentiable spike, and only slight differences in the moderately clear and not clear categories. Two good examples of human subject power spectra demonstrating the clear and not clear categories were chosen.

Insert Figure 15 Here

The accuracy spectrum for Subject 25 (Figure 15) is an excellent example of a clear spectrum. Primary spikes are easily distinguishable from noise. While many subjects had spectra which were clear, very few provided this degree of clarity. Figure 16 shows a good example of a Not Clear power spectrum. The RT data for Subject 35 displays a large number of high spikes in the frequency bands of the spectrum where the majority of our data resided. This made identification of primary frequencies exceedingly difficult.

Insert Figure 16 Here

Our observations of primary frequency power suggest that there isn't a major difference between accuracy and RT data in that area. When examining subject groups by power it was noted that for group 1, poor performers, only 7 of 
the 12 subjects had good power, 3 of the 11 subjects in group 2 had good power. Only 3 of the 9 in group 3 had good power, and 1 of 8 in group 4 , the good performers, showed good power in both spectra. In general, the level of power decreased with group.

Also compared was overall noise level in accuracy and RT power spectra. Table 18 shows the percentage of subjects who fell into each of our three noise rating categories for both accuracy and RT.

Insert Table 18 Here

Across the board, the ratings of noise level for accuracy spectra are higher than those of RT spectrum with the largest difference being that $15 \%$ more subjects rate at the medium noise level for accuracy spectra. $20 \%$ of accuracy spectra showed more noise than their matched RT spectra. Again two good examples demonstrating the opposite ends of the noise spectrum were chosen.

Insert Figure 17 Here

Figure 17 shows a good example of a low noise level. Note that the highest noise spikes are not equal to $1 / 2$ the power of the 1 primary frequency identified. Low noise levels often, but not always, equated to high primary frequency clarity. 


\section{Insert Figure 18 Here}

The RT spectrum is shown for Subject 24. (See Figure 18) This spectrum demonstrates high overall noise level. Note that the majority of the noise occurs in frequency bands greater than 30 second periods. This might suggest multiple overlapping frequencies which cannot be differentiated by this process. The end result is, however, that the primary frequencies are most often masked by the high level noise. In this way, RT data seems to be $20 \%$ more useable than accuracy data. Noise was not assessed by subject group.

The final comparison of RT and accuracy spectra involved the assessment of similarity between the actual identified frequency bands for each subject's accuracy vs RT spectra. Table 19 shows the results of our observations.

Insert Table 19 Here

It is important to know first that all spectra showed some level of similarity. There were no cases where at least one of the major spikes on the spectra did not coincide. Therefore, when considering the results for this observation, keep in mind that they are relative and not absolute. The results of the observations suggest that in the vast majority of the cases, spectra are more similar than not. In almost half of the cases the primary frequencies were evident, if not identified, for both accuracy and RT. 
Figure 19 in a good example of spectra that were considered the same for the purpose of this observation. Note that 2 of the 3 identified frequency bands were identical, and that the third was only slightly shifted. Many subjects showed similar spectra, but few were quite as clearly defined. The rating of comparability of primary frequencies proved to be a challenge. We show an example of spectra rated different in Figure 20.

Insert Figure 20 Here

Several additional primary frequencies were identified for the RT function. Although one frequency is the same between the two spectra, they are not similar enough to rate in that way.

Examining the spectra by group, we greater differences when looking at only subjects with good power. These differences were most evident in group 1. When observing all 12 subjects in the group regardless of power, of the top 2 peaks, 6 had both in common, 5 had one in common. The positioning of peaks was reversed for some subjects. When looking at good power only, 6 of 7 had both in common, and 1 had only 1 in common. Group 2 was made up of below averge performers. Of 11 subjects 3 shared both top powers, and 8 shared 1 . Six of the subjects had the same top power. Of the 3 subjects with good power, 1 shared both peaks, and 2 shared 1. Group 3 was made up of above averge performers. Of 9 subjects 4 shared both top powers, and 3 shared 1 . Of the 3 
subjects with good power, 2 shared both peaks, and 1 shared 1 . Group 4 was made up of above good performers. Of 8 subjects 3 shared both top powers, and 4 shared 1 . Only one subject had good power. The overall power for group 4 was very low.

The results of our observations of accuracy and RT power spectra were conclusive. RT seemed to provide a function with fewer cases of high or moderate noise than accuracy, but in terms of the measures which were very important to research, RT and accuracy seemed to provide functions that were equally as clear to interpret. Most importantly, they seem to provide very similar frequency band data in nearly all cases. Based on these findings, RT could not be chosen over accuracy as a preferred dependent variable for identifying fluctuations in human responding. This determination was made based on the selection of the best case from each of our observations. RT and accuracy are comparable in each case except overall noise, where RT provides a clearer function. No advantage in terms of ease of identification or accuracy of primary frequency identification was indicated, however.

Experiment 3 Part 3: RT/FFT comparison of Good and Poor Human Accuracy Responders

\section{Procedure}

FFT data for human subject RT from Experiment 3, Part 1, was broken down into 4 groups by subject performance level (Hit Rate) as defined in Experiment 1, Part 1. Percent of bin membership was calculated for each subject group, and the groups were compared for each bin with a Two-way Chi 
Square (see Appendix $M$ for Chi Squares). Percent group membership within bins in Table 20.

\section{Insert Table 20 Here}

\section{Results, Experiment 3 Part 3}

Results of the Chi Square test run between subject accuracy level groups for RT FFT bins $1,2,3 \& 4$ were not significant. For bin $1, X^{2}(3)=0.079, \underline{p}>.05$; $\operatorname{Bin} 2 X^{2}(3)=1.336, p>.05 ; \operatorname{Bin} 3 X^{2}(3)=0.806, p>.05 ; \operatorname{Bin} 4 X^{2}(3)=3.912, p$ $>.05$

\section{Discussion, Experiment 3 Part 3}

The purpose of Part 3 was to check for differences in periodicity between subjects of different accuracy levels for periodicities derived from RT FFT results. This result is similar to that obtained for accuracy FFT data with the same subjects. The results suggest that there are no significant differences in RT response periodicity based on the subjects overall level of performance on the CPT. Thus, there is no way to differentiate good performers from poor based on RT periodicity.

\section{General Discussion for Experiment 3}

This experiment was meant to investigate another avenue of investigating the same questions that were researched in Experiment 2 by providing a momentary measure of CPT performance using reaction times. In 
addition, the relationship between accuracy and reaction time spectra was explored. It was discovered that accuracy and RT are similar in terms of periodicity, and that for RT, the dominant wave bands do not differ depenent upon the performance level of that subject. Evidence of a slight advantage for using the reaction time function over accuracy was discoered. This advantage was experienced more in terms of creating a noise free function than an actual advantage in identification of frequency bands. When you also consider that $\mathrm{RT}$ is more robust against ceiling effects, leaving it free to fluctuate with any underlying processes even when consecutive hits are being generated, and that there are no apparent disadvantages to using the RT measure, RT becomes the logical choice for examining fluctuations in human perfornace on a CPT. This finding is significant because accuracy is widely used in the field today. How does one use the reaction time measure to give a general rating of performance? Is there a general lengthening or shortening of reaction time over the course of a CPT? These are questions that remain unanswered, but which may prove to be revealing in the continuing search for a better measure of attention. 


\section{Study Summary and Conclusions}

This work can be viewed as having three major components. The first was the investigation of methods to describe and quantify fluctuations of good performance in CPT data. The results of that investigation were two different techniques, one which examined the number and length of hit runs in the data, and the second which examined response data via a spectral analysis process. Each method described and or quantified data in an interesting but different way. Where applicable, the methodologies were thoroughly tested to assess whether they would either introduce artifacts or distort real fluctuations. The positive results obtained during the examination and testing of these techniques were a strong indicator of the validity of these techniques for identification and description of fluctuations in performance.

The application of those techniques to real CPT data was the second major component. That investigation included description of the nature of runs of good performance and the identification and description of the presence and distribution of periodicity in real CPT data. Differences between people of different competencies were also assessed, and two different dependent measures were used where possible. General findings suggested that runs and periodicity were detectable in subject performance, and that there were minimal differences in the nature of these fluctuations between subjects of iiffering ability.

The third component of this work comprised the validity testing of the techniques developed in earlier components. Methods for examining the origins of fluctuations in CPT performance were designed and implemented. 
The primary question addressed by these methods was, were the findings in part 2 attributable to some fluctuation in an attention mechanism, or were they due to some random factor or some artifact of test structure (i.e. patterning of targets in the stimulus template; more or less difficult targets to differentiate EC-C vs. A-G-G which might promote false alarms or misses on certain targets). The methodology involved the creation of simulated data sets, essentially by taking actual subject data and re-assigning hits and misses to different targets, thereby leaving total percent accuracy constant. Simulated data sets were matched to subject performance categories to minimize the difference between the number of errors in the function. Quantitative and qualitative comparisons between human and simulated subjects failed to provide firm evidence of differences between the two groups. These findings brought into question the utility of the CPT/MAW/FFT method for the identification and quantification of fluctuation in performance on a CPT. The results were not conclusive, however. Possible explanations for the results were that the CPT/MAW/FFT method was insensitive to such difference, that the analysis methods (Chi Square) used were insensitive to the small differences in the data, or that the CPT design forces periodicity into the records which is reflected in both real and simulated data. As an example of the latter, it was observed that performers who made very few errors, would have long periodicities. The origin of these periodicities could be real, due to a real, oscillating attention mechanism. It was also noted, however, that when a simulated record was created with only a few errors, the placement, though random, would almost inevitably result in long periodicities. Thus, by design, such simulated data sets would show a resemblance to the real subject data. 
Despite the lack of clarity in some areas, the first general hypothesis of this dissertation was supported by the results. There was strong evidence that the ability to sustain attention as measured by a CPT does not simply decline in a linear fashion, but fluctuates between good and poor performance. In addition, it was noted that although there were always periods of poor performance, these were not always more evident at the end of a trial. Thus, it was possible to have little or no decline in performance if this was calculated by examining only the beginning and ending of a specific trial. The second general hypothesis was only partially supported, and it remains unclear if the CPT provided the sensitivity required to distinguish non-random fluctuations in performance. Methodologies developed to quantify fluctuations were successful with CPT data types, however. Additional investigation is required in this area. Likewise, support for the general predictions of the dissertation was inconclusive.

The many intricacies of data generated by the CPT bring about new questions and related problems. Oscillations were evident in performance data, but the investigations into the source of the oscillations were inconclusive. What was the cause of the observed fluctuations in performance? Was it driven by random forces, or perhaps an expectancy effect? Makeig, Gilden and Conte all reported fluctuations in responding, and yet no physiological or behavioral theory had yet been presented which would describe the behavior which each of those researchers and this author have witnessed. Simple learning theory was ruled out. There was no evidence of hit chaining caused by excitatory effects of making a correct response. There was some evidence of an expectancy effect which seemed to drive subjects back to good responding, but 
some mechanism caused a decrement in performance to begin with. Tucker and Williamson (1984) have proposed a lateralized system of redundancy and habituation by which the left and right sides of the brain constantly battle to focus and to habituate ongoing neural operations. Tucker and Williamson have related this system to the attentional system. Theoretically speaking, behavior moderated by such a system would fluctuate rather than decline in a linear fashion. Such a system might explain the the non-periodic fluctuations reported by Makeig and Inlow (1993), but the theory provides no mechanism for periodic changes in attentional behavior. The results of this study and those reported by Conte et. al. (1995) suggest that there is periodicity to fluctuations in performance. Gilden and Gray-Wilson (1995a; 1995b), examined several theoretical models which they hoped would provide insight into the nature of fluctuations in attention and skilled performance. Through numerical simulation and empirical study, they were able to determine that streaky performance which they observed most closely resembled a wave-modulation model in which two distinctive systems independently exercise control over attention. The first system was associated with early sensory and perceptual processing. In that system, they posited small wave-like variations. The second system was associated with controlled attention. They posited that this second system was not periodic, but fluctuated randomly over time depending on cognitive and somatic factors. They concluded that periodic oscillations in performance were most clearly seen in tasks that were preattentive, requiring little or no focused attention. Additionally, they suggested that, in tasks requiring focused attention, these oscillations were still within the function, but were masked by fluctuations in attentional resources. This model seems well suited to the results of this 
study, except that it does not explain long interval fluctuations over 100 seconds. Thus, despite Gilden and Gray-Wilson's clear findings, we are left without a model that captures the nature of the observed results. Consequently, although we can claim that fluctuation and even periodic fluctuation exists in the results of the CPT, the nature and cause of those fluctuation, and the clinical and theoretical implications of those fluctuations remain as unanswered questions. 


\title{
Appendix A
}

/*

\begin{abstract}
\#include <stdio.h> \#include <stdlib.h> \#include <string.h> \#include <ctype.h> \#include <time.h> \#include "dutils.h"
\end{abstract}

$\begin{array}{lrr}\text { \#define MINUTES } & 3 \\ \text { \#define SECONDS } & 2 \\ \text { \#define MILLISECONDS } & \text { I } \\ \text { \#define POSITION } & 0 \\ \text { \#define TB } & 9 & \end{array}$

\#define OUT_TME( $(t)$ (ind $x==$ POSITION ? $(t): 1$

(ind $x==$ MILLISECONDS ? $((t)-1)^{*}$ isi : 1

(ind $x==$ SECONDS ? $(($ float $)(((t)-1) *$ isi $) / 1000): 1$

$((($ float $)(((\mathrm{t})-1) *$ isi $) / 1000) / 60))))$

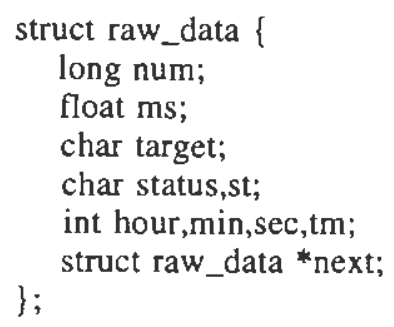

FILE * $\log f=0,{ }^{*}$ wprfl=0, ${ }^{*}$ pprfl=0, ${ }^{*}$ ltfl $=0$;

float weight[16];

long crit $=500$,isi $=0$, midp $=0$;

int $\sin =0$, ind $=0$, $w$ width $=0$, wadvance $=1$, mark $=$ FALSE, divide $=$ TRUE, ind $x=$ POSITION; int fftstep $=0$, fftwin $=0$; int data_start $=0$,data_end $=0$; int react_time $=$ FALSE;

struct raw_data *read_raw(FILE *fl);

struct raw_data *extract_targets(struct raw_data *raw); char *parse_header(FILE *f1);

char primary $[500]=" 10 "$, critical $[500]=" 10 "$, window $[500]=" 10 "$;

char lentime $[500]=" 10 "$; 
char mid_point[500]="10";

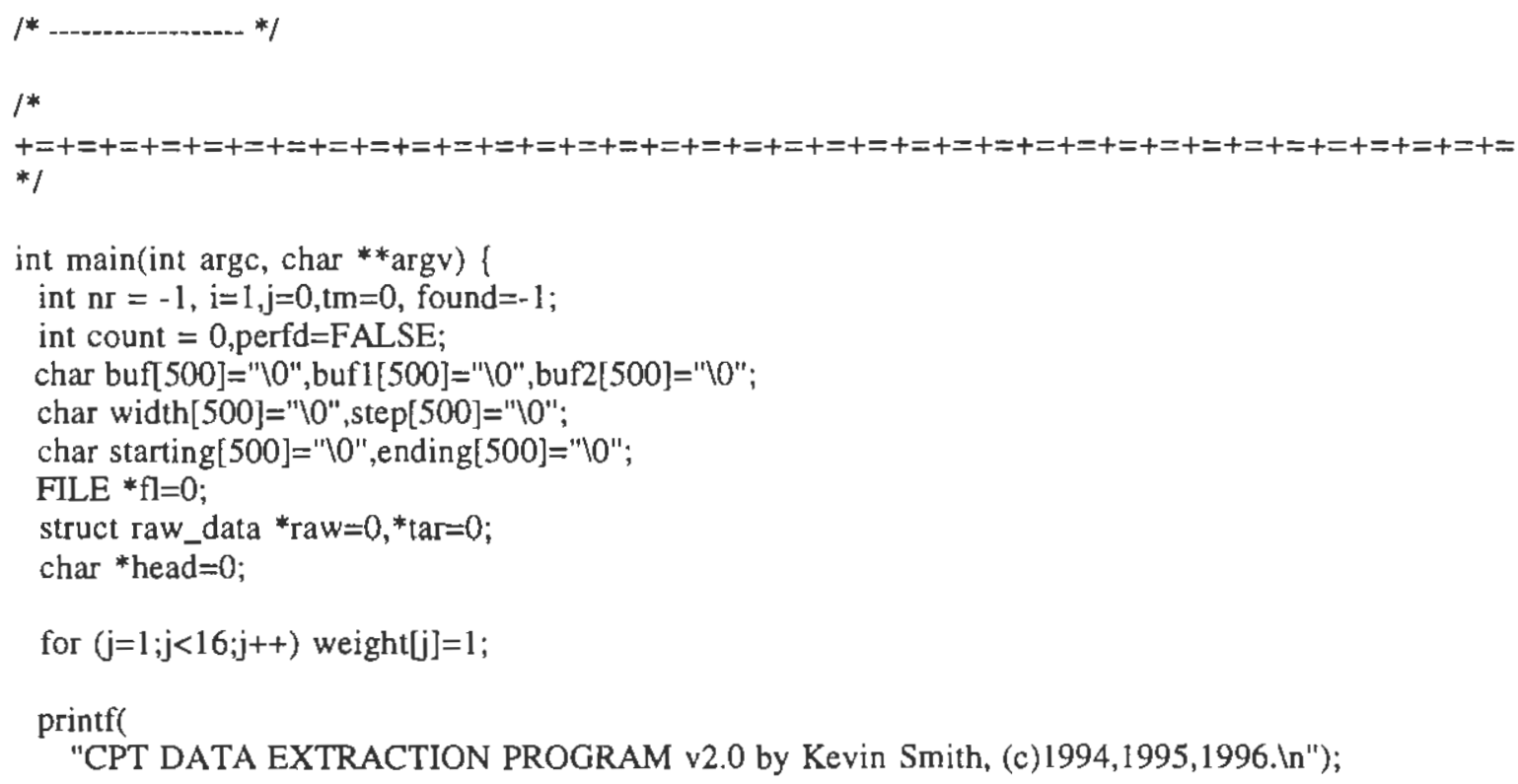


case ' $\mathrm{G}$ ':

case ' $\mathrm{g}$ ':

colon_chop(argv[i],buf,critical);

colon_chop(critical,starting,ending);

if(!*critical II (data_start=atoi(starting) $)<1 \|$

(data_end=atoi(ending) $<0)\{$

printf("Error: Starting Stimulus or Ending Stimulus missing.ln",buf);

printf("Usage -G:Starting \#:Ending \#(n");

printf(" (in \# of stimuli) [Starting >=1] [Ending $>=1$ or 0 for Last]) $\backslash n "$ );

exit $(0)$;

\} else

sprintf(critical,"\%d",data_end);

of the data");

printf("- Only stimuli \%d to \%s will be included.In",data_start,data_end?critical:"the End

break;

case ' $O$ ':

case ' 0 ':

colon_chop(argv[i],buf,primary);

if(!*primary) !

printf("Error: No output filename with (\%s) \n",buf);

printf("Usage -O:FILENAMEไn");

exit $(0)$;

\} else [

printf("- Psimary output filename will be (\%s) \n",primary);

printf(" The extention .PPO will be appended to Performance data. $n ")$;

l

printf(" The extention .MPO will be appended to MER data.ln");

break;

case 'I':

case ' $\mathrm{i}$ ':

printf("- Individual output files will be generated.ln");

printf(" The extention .prf will be appended to Performance data.ln");

printf(" The extention .mer will be appended to MER data. $(n ")$;

ind = TRUE;

break;

case ' $S$ ':

case 's':

printf("- Data set will be simulated. Target only input files. In");

sim = TRUE;

break;

case ' $\mathrm{R}$ ':

case ' $r$ ':

printf("- Individual Reaction Time files will be generated. $\ln ")$;

printf(" The extention , art will be appended to Performance data. $\mid n ")$;

printf(" The extention .wrt will be appended to MER data. $\ln ")$;

printf(" The extention .ffr will be appended to MER data.ln");

react_time $=1$;

break;

case ' $\mathrm{M}$ ':

case ' $m$ ':

printf("- Data analysis will begin at MARK.In");

mark = TRUE;

break;

case ' $\mathrm{D}$ ':

case 'd':

printf("- Window totals will NOT be divided by \# of targets in the window. $n "$ ");

divide = FALSE;

break; 


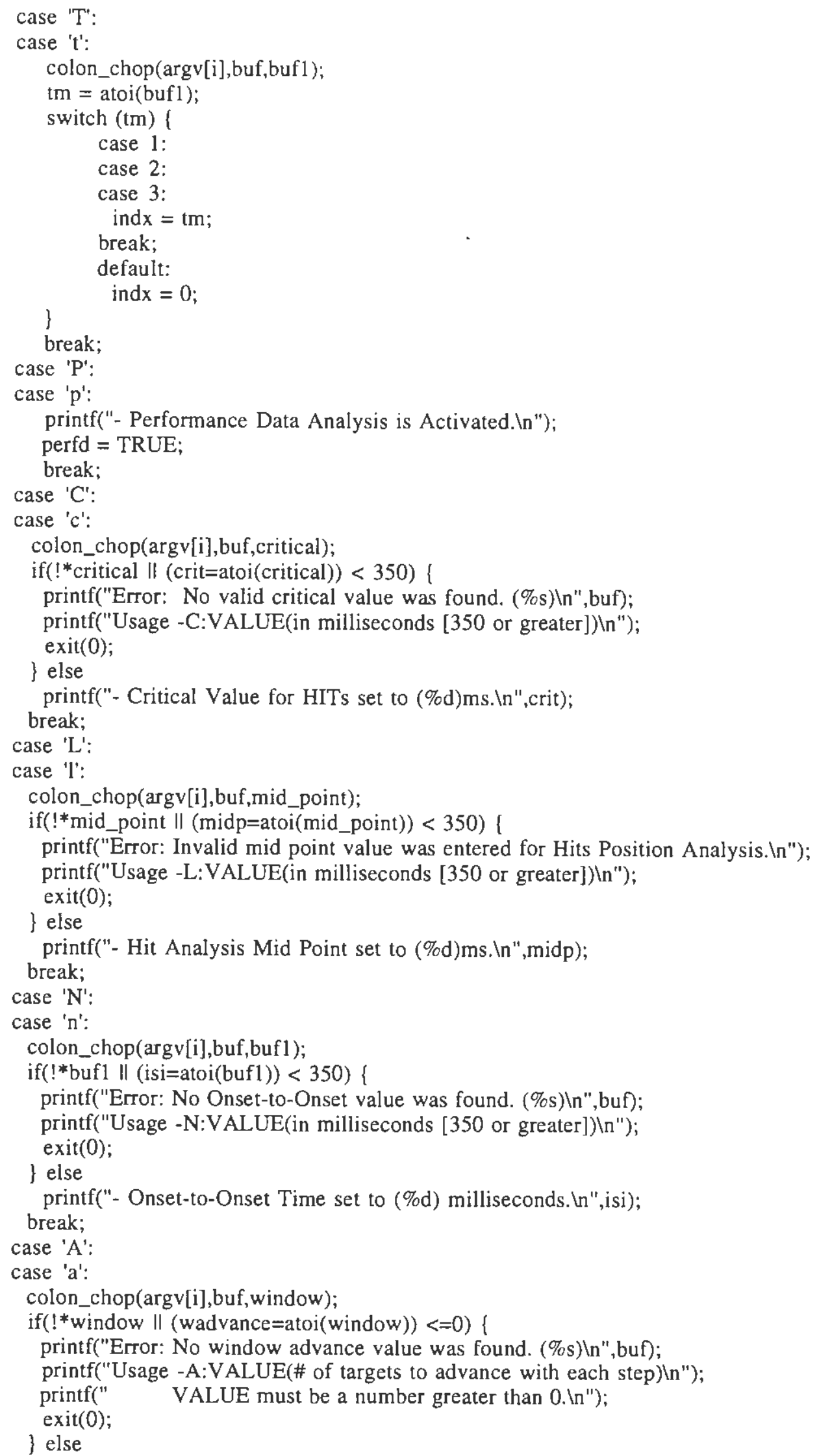




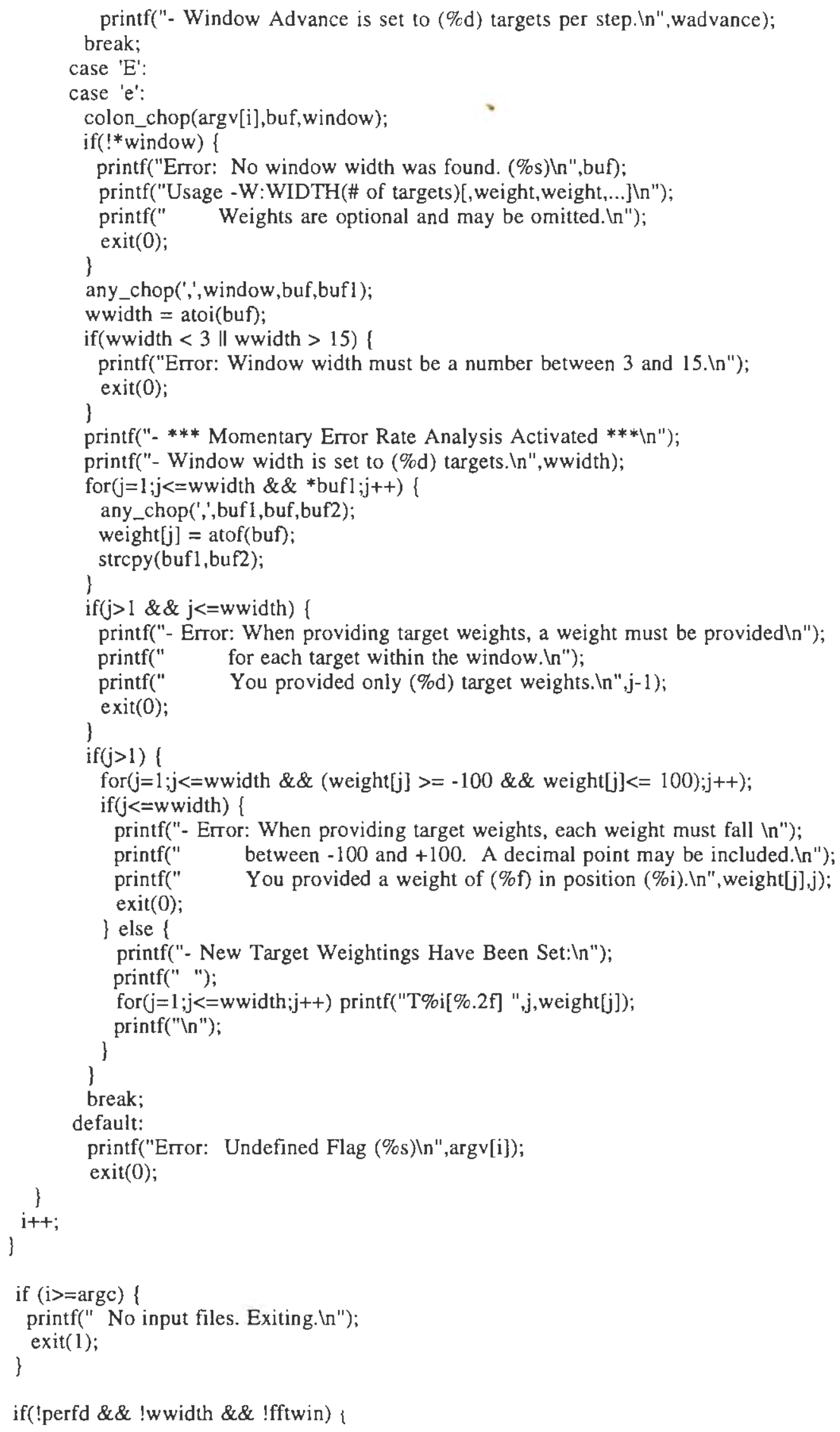


printf(" No data analysis has been selected. Exiting. ln");

exit(1);

\}

switch(ind $x)$ |

case 1:

printf("- Targets will be indexed by time in milliseconds. In"); break;

case 2:

printf("- Targets will be indexed by time in seconds. n" $^{\prime \prime)}$;

break:

case 3:

printf("- Targets will be indexed by time in minutes. $\ln ")$;

break;

default:

)

printf("- Targets will be indexed by time as stimulus \#. In");

if(*primary) \{

if(perfd) \{

sprintf(buf," \%s.PPO",primary);

if(!(pprfl=fopen(buf, "w+"))) (

printf(

"Error: Unable to create Performance Primary Output file. Exiting. In");

) exit(1);

fprintf(pprfl,"'nIDlt\#Targetsit\#HitsltAV.HTtHitRateltTot.Stimlt\#FAltFARatelt\#Misseslt\#LateHitsitAV.

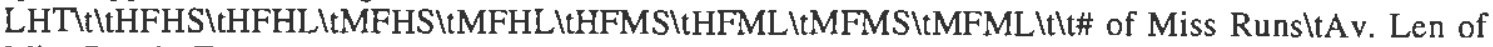
Miss Run in Trgs. ltAv. Len of Miss Run in Secondslttt\# of Hit RunsitAv. Len of Hit Run in Trgs.ttAv. Len of Hit Run in SecondsthttAv. Time Between Targets(Sec)htMinimum Time Between

Targets(Sec)ltMaximum Time Between Targets(Sec)ไn");

\}

if(wwidth) \{

sprintf(buf,"\%s.MPO",primary);

if $(!($ wprfl=fopen(buf,"w+"))) !

printf(

"Error: Unable to create Momentary Primary Output file. Exiting. ln");

exit(1);

if(*lentime) 1

sprintf(buf,"\%s.int",lentime);

if(!(pltfl=fopen(buf,"w+"))) !

printf("Error: Unable to create Interval Output file. Exiting.ln");

\} exit(1);

if(!ind)

printf("** Warning: Demographic information for individual subjects is NOT saved.In");

for $(i ; i<\operatorname{argc} ; i++)$ l

printf("-- Current Work File (\%s) --ln", argv[i]);

any_chop('/",argv[i],buf,buf1); 


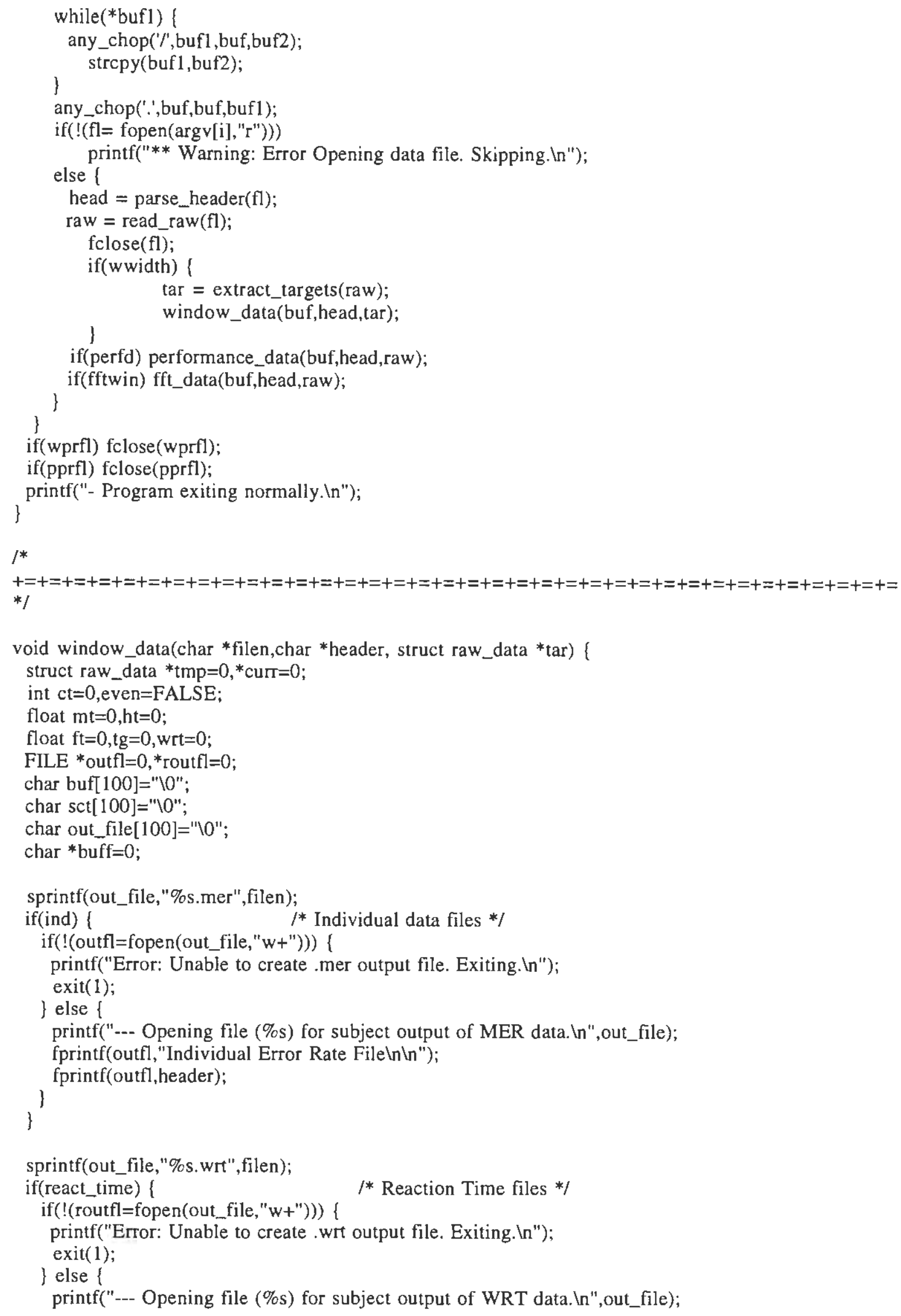




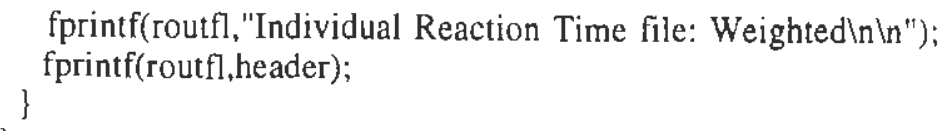

fprintf(routfl,"Individual Reaction Time file: Weightedlnไn");

printf("--.- Moving MER window, Width(\%d) Step(\%d)ไn",wwidth,wadvance);

even $=$ FALSE;

if(odd_number(wwidth))

$\operatorname{tg}=((($ float $)$ wwidth $/ 2)+.5)$;

printf("-.-- The Window width is an ODD number. (\%f) $\backslash n$ ",tg);

\} else \{

even $=$ TRUE:

$\operatorname{tg}=($ wwidth $/ 2)$

\}

printf("-..-- The Window width is an EVEN number. (\%d) $\backslash n "$,tg);

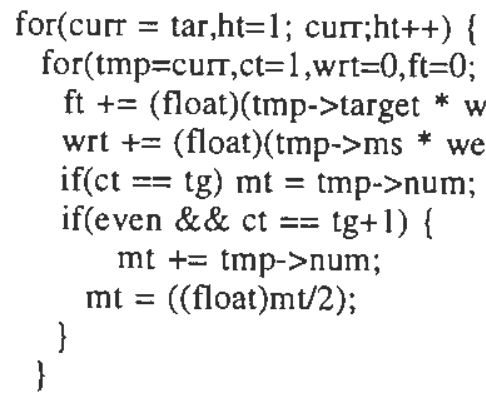


out_file);

1

\section{if(react_time)}

sprintf(out_ffle,"\%s.art",filen); /* Actual Reaction Time Data */

if $($ ! (artfl=fopen(out_file,"w+"))) \{

printf("Error: Unable to create .art output file. Exiting.ln"); exit(1);

I else \{

printf("--- Opening file (\%) for output of raw RT data.ln",out_file);

\}

\}

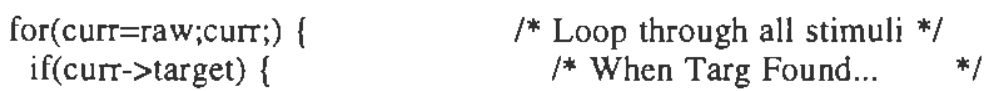

$\mathrm{ct}++; \mathrm{ft}=\mathrm{FALSE} ; \mathrm{msc}=0 ;$ resp=FALSE;

tc $=$ curr->num;

tct $=$ curr $>$ num $*$ isi;

while (curr \&\& (msc $<=$ crit)) $\{/ *$ Loop while curr and TIME $<$ crit $* /$

if(!msc $\|$ !curr->target) \{

if $($ curr $>\mathrm{ms}>0)\{\quad / *$ If there was a response this Stim */

msc $+=(($ float $)$ curr $->\mathrm{ms} * 1000) ; \quad / *$ Add Resp Time to $\mathrm{msc} * /$

if $\left(\right.$ (curr->target \& \& curr->status $\left.=={ }^{\prime} \mathrm{H}^{\prime}\right)$ If ! curr->target $)$ (

st++; $\quad 1 *$ Increment Stimulus Counter *f

curr $=$ curr $\rightarrow$ next; $\quad l^{*}$ Increment curr CT within Time Loop */

resp = TRUE;

break;

\} else \{

\}

f else ?

$\mathrm{msc}+=\mathrm{isi}$;

\}

\} else $\left\{\quad f^{*}\right.$ When msc $>0$ and curr stim is a Targ *f

printfe

/* Force Exit from Time COunt Loop */

1* If there was NO response to curr stim */

$l^{*}$ Increment msc by onset-to-onset time */

"-- Target \#\%d - Warning: Target interference. Shorten critical time.ln",ct); printf(

Data for inital target has been lost.(n");

$\mathrm{ft}=\mathrm{TRUE}$

ct--;

break;

\}

st++;

curr=curr->next; $\quad / *$ Increment Curr Stim CT within time count Loop */

\} /* End time count Loop (accumulated time < crit) */

if $(\mathrm{msc}<=300)\{$

fa+t;

avfa $+=($ tct-tolt $)+\mathrm{msc}$;

ms++;

/* -.- Begin Runs Analysis Following a Miss -.- */

switch(lpst) \&

/* Look at Lapse Data after a MISS */

case 0
/* Flag as Faulty, Do NOT inc curr */

/* Decrement Targ CT */

/* Force Exit from Time COunt Loop */
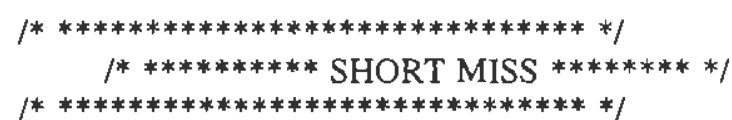

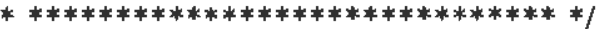




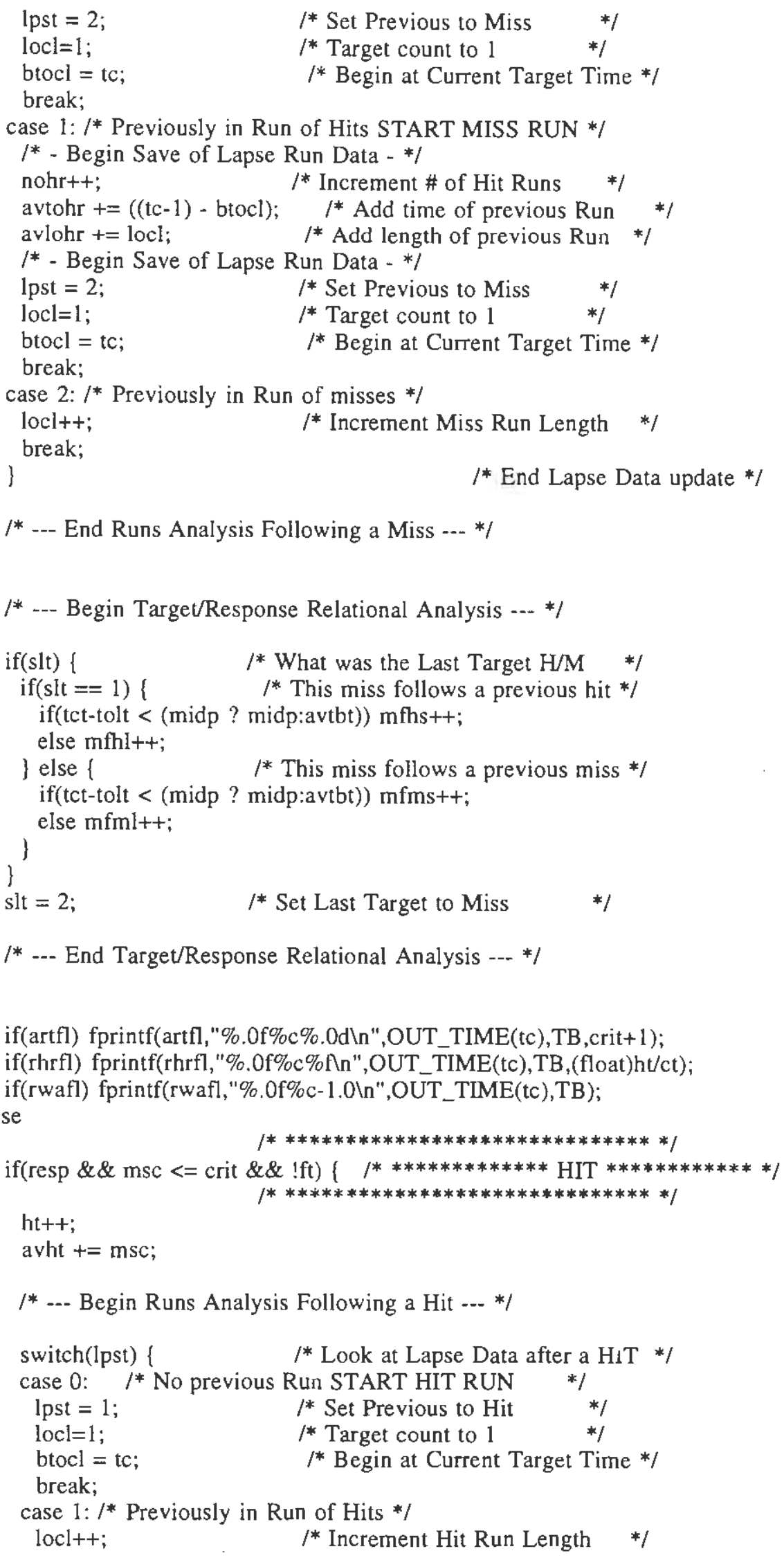


break;

case 2: /* Previously in Run of Misses START HIT RUN */

/* - Begin Save of Lapse Run Data - */

nolr++; $\quad l^{*}$ Increment \# of Lapse Runs */

avtolr $+=(($ tc- 1$)-$ btocl $) ; \quad I^{*}$ Add time of previous Run */

avlolr $+=$ locl; $\quad I^{*}$ Add length of previous Run */

$1^{*}$ - End Save of Lapse Run Data - */

Ipst $=1 ; \quad$; Set Previous to Hit */

locl=1; $\quad 1 *$ Target count to $1 \quad * /$

btocl = tc; $\quad 1 *$ Begin at Current Target Time */

break:

)

/* ..- End Runs Analysis Following a Hit ... */

/* ..- Begin Target/Response Relational Analysis -.. */

if(slt)

if(slt ==1) $\left(\quad /^{*}\right.$ This hit follows a previous hit */

if(tct-tolt < (midp ? midp:avtbt)) hfhst+;

else hfhl+t;

\} else ( $\quad 1 *$ This hit follows a previous miss */

if(tct-tolt < (midp? midp:avtbt)) hfms++;

else hfml++;

1

1

slt $=1 ; \quad \quad \quad /^{*}$ Set Last Target to Hit $\quad$ */

/* -.- End Target/Response Relational Analysis --- */

if(artfl) fprintf(artfl,"\%.0f\%c\%.ofin",OUT_TIME(tc),TB,msc);

if(rhrfl) fprintf(rhrfl," \%.0f\%c\%fn",OUT_TIME(tc),TB,(float)ht/ct);

if(rwafl) fprintf(rwafl, "\%.0f\%c l.O\n",OUT_TIME(tc),TB);

\} else I

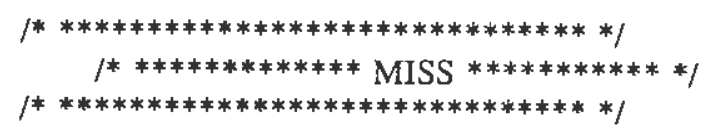

$\mathrm{ms}++$;

$/^{*}$--- Begin Runs Analysis Following a Miss --- */

switch(lpst) \{ /* Look at Lapse Data after a MISS */

case 0: $\quad I^{*}$ No previous Run START MISS RUN $\quad * /$

lpst $=2 ; \quad$ * Set Previous to Miss *j

loclel;

1* Target count to 1 */

btocl $=\mathrm{tc} ; \quad \quad / *$ Begin at Current Target Time */

break;

case 1: $/ *$ Previously in Run of Hits START MISS RUN *

/* - Begin Save of Lapse Run Data - */

nohrt+;

/* Increment \# of Hit Runs */

avtohr $+=(($ tc- 1$)-$ btocl $) ; \quad / *$ Add time of previous Run */

avlohr $+=$ locl; $\quad 1 *$ Add length of previous Run *j

$/ *$ - Begin Save of Lapse Run Data - */

lpst $=2$;

locl $=1$;

btocl $=\mathrm{tc}$;

/* Set Previous to Miss */

break;

I* Target count to 1 */

/* Begin at Current Target Time */ 
fprintf(pprfl,"\%ilt",ct);

fprintf(pprfl,"\%ilt",ht);

fprintf(pprfl,"\%.3ftt",avht);

fprintf(pprfl,"\%.3ftt",((float)ht/(ct?ct:1)));

fprintf(pprfl, "\%ilt",st);

fprintf(pprfl,"\%ilt",fa);

fprintf(pprf," \%.3ft", ((float)fa $/ 1$

$\left(\left((\right.\right.$ float $)\left(\right.$ st $\left.\left.-\left(\mathrm{ct}^{*}(\mathrm{crit} / \mathrm{isi})\right)\right)-1\right)>0$ ? ((st - (ct*(crit/isi $\left.\left.\left.\left.\left.\left.)\right)\right)-1\right): 1\right)\right)\right)$;

fprintf(pprf,,"\%ilt",ms);

fprintf(pprfl,"\%ilt",lh);

fprintf(pprfl," \%.3fit",avlh);

fprintf(pprfl,"lt\%ilt\%ilt\%ilt\%ilt\%ilt\%ilt\%ilt\%ilt",

hfhs, hfhl,mfhs,mfhl,hfms,hfml,mfms,mfml);

fprintf(pprfl,"tt\%itt\%.3ftt\%.3nt",nolr,

((float) avlolr/(nolr > 0?nolr:1)),

(((float) avtolr/(nolr > 0?nolr:1))*isi/1000));

fprintf(pprf, "lı\%ilt\%.3ft\%.3ft",nohr,

((float) avlohr/(nohr > 0?nohr:1)),

((float) avtohr/(nohr > 0?nohr:1))*isi/1000)); \}

fprintf(pprf, "lt\%.3fto.0ft\%.0fla",avtbt/1000,mintbt/1000,maxtbt/1000);

printf("-- PRF Summary: \%d Stimuli \%d Targets \%d Hits \%d Misses \%d FAln

$\% \mathrm{~d}$

Miss Runs \%d Hit Runsin",st,ct,ht,ms,fa,nolr,nohr);

\}

$i^{*}$

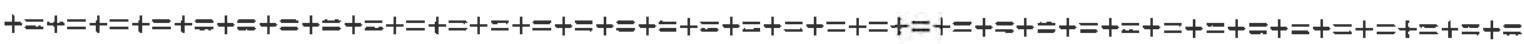

$* /$

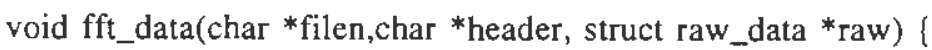

struct raw_data ${ }^{*} \mathrm{c} t=0,{ }^{*}$ curr $=0$;

FILE *outfl $=0, *$ outfl $1=0$;

float tct $=0$;

float $\mathrm{msc}=0$;

int $s t=0, h t=0, f a=0, m s=0, l h=0$;

int $\mathrm{ft}=0$, resp $=0$, slt $=0$;

char out_file[1000]="10";

printf("-- Beginning Movement of Averaging Window...ln");

if(ind) $\left\{\quad l^{*}\right.$ Individual data files */

sprintf(out_file,"\%s.ffa",filen);

if (! outfl=fopen(out_file,"w+")) ) (

printf("Eror: Unable to create .ffa Accuracy output file. Exiting. $\left.\ln ^{\prime \prime}\right)$;

exit(1);

f else ( \}

printf("--- Opening file (\%s) for output of Accuracy FFT data.in",out_file);

\section{if(react_time)}

sprintf(out_file,"\%s.ffr",filen);

if(!)(outfl1=fopen(out_file,"w+")) \{

printf("Error: Unable to create .ffr RT output file. Exiting. In");

exit(1);

$)$ else \{

printf("--- Opening file (\%s) for output of RT FFT data.ln",out_file); 
)

curt = raw;

while(curr) \{

if(curt->target) !

ct = curr;

$\mathrm{ft}=\mathrm{FALSE} ; \mathrm{msc}=0 ;$ resp $=0$;

while(cur \&\& (msc <= crit)) !

if(!curr->ms) $s t++$;

if(!msc II !curr->target)

if (cur $>>\mathrm{ms}>0)$ !

msc $+=$ ((float)curr->ms * 1000);

if ( (curr->target \&\& curr->status $\left.=={ }^{\prime} \mathrm{H}^{\prime}\right) \|$ !curr->target $)$ ( curT=curr->next;

résp = TRUE;

break;

\}

else

$\mathrm{msc}+=\mathrm{isi}$;

j

| else

printf("-- Warning: Target interference. Shorten critical time. In");

printf(" Data for inital target has been lost.ln");

$\mathrm{ft}=\mathrm{TRUE}$

break;

curr=curr->next;

if(msc $<=300)\left\{\quad /^{*} \ldots\right.$ This is a SHORT FA - * $^{*}$ /

ct->st $=0$;

$\mathrm{ct}->\mathrm{tm}=\mathrm{crit}$

\} else

if(resp \&\& msc <= crit \&\& !ft)

$\mathrm{ct}->\mathrm{st}=1$

$\mathrm{ct}->\mathrm{tm}=\mathrm{msc}$ :

\} else $\left\{\quad /^{*}\right.$-.- This will be a MISS or no-response --- */

$\mathrm{ct}->\mathrm{st}=0$ :

$\mathrm{ct}->\mathrm{tm}=\mathrm{crit}$

\}

J else

curr $->$ st $=0$;

curr- $>\operatorname{tm}=0$

printf("--- Compiling MAF data for Accuracyln");

for (ms $=0$, tct $=$ fftwin +1 , curr=raw; curr \& \& tct $>$ fftwin;)

for $(\mathrm{fa}=0, \mathrm{ht}=0, \mathrm{ct}=\mathrm{curr}, \mathrm{tct}=1 ; \mathrm{curr} \& \& \mathrm{ct} \& \& \mathrm{tct}<=\mathrm{fftwin} ; \mathrm{ct}=\mathrm{ct}->$ next, $\mathrm{tct}++)$

$h t+=c t->s t$;

if(ct->target) fa+t;

if(tct $>$ fftwin) 1

ms++;

printf("Point \%d: \%d Targets \%d Hits Score: \%fln", 
fprintf(outfl,"\%fln",((float)ht/fa));

$$
\text { Ins, fa, ht, ((float)ht/fa)); */ }
$$

\} ) for $(\mathrm{lh}=1$; curr \&\& $\mathrm{lh}<=$ fftstep;cur=curr->next,, $\mathrm{h}++)$;

if(outfl) fclose(outfl);

if(outfl1) \{

printf("--- Compiling FFT data for Reaction Timeln");

for(ms=0,tct=fftwin +1 ,cur=raw;curr $\& \&$ tct $>$ fftwin;) (

for $(\mathrm{fa}=0, \mathrm{ht}=0, \mathrm{ct}=\mathrm{curr}, \mathrm{tct}=1 ; \operatorname{curr} \& \& \mathrm{ct} \& \& \mathrm{tcl}<=\mathrm{fftwin} ; \mathrm{ct}=\mathrm{ct}->\mathrm{next}, \mathrm{tct}++)$

$h t+=c t->t m$;

\}

if(ct->target) fa++;

if $($ tct $>$ fftwin $)$ \{

ms++;

/* printf("Point \%d: \%d Targets \%d Hits Score: \%fln",

1

fprintf(outfl1,"\%)n",((float)ht/(fa?fa:1)));

ms,fa,ht,((float)bu/(fa?fa: 1$))) ; * /$

\}

for(lh $=1$; curr $\& \&$ lh $<=$ lftstep;curr=curr- $>$ next, lh ++ );

)

if(outfl1) fclose(outfl1);

\}

return;

$l^{*}$

$+=+=+=+=+=+=+=+=+=+=+=+ニ+=+$ */

struct raw_data *extract_targets(struct raw_data *raw) (

struct raw_data $*$ top $=0,{ }^{*}$ bott $=0,{ }^{*}$ tmp $=0,{ }^{*}$ curr $=0$;

long num $=0, \mathrm{msc}$;

int ht $=0, c t=0$;

int $\mathrm{ft}=0$, resp;

char buf[ 1000]="ไ0";

for(curr=raw;curr;) \{

if(curr->target) ?

CREATE(tmp,struct raw_data, 1);

if(!tmp) \{

printf("Error: Unable to allowcate RAM for target data structure.ln");

) exit(1);

ct++;

tmp->num $=$ curt $\rightarrow>$ num;

tmp->hour $=$ curr $->$ hour;

tmp->min = curr $>>\min$;

tmp->sec $=$ curr $->\mathrm{sec}$;

tmp->target $=0$;

$\mathrm{ft}=$ FALSE;msc $=0 ; \mathrm{resp}=0$;

while(curr $\& \&$ (msc $<=$ crit)) \{

if(!mse II !curr->target $)$ \{

if (curT->ms $>0$ )

$\mathrm{msc}+=(($ float $)$ curr->ms $* 1000)$; 


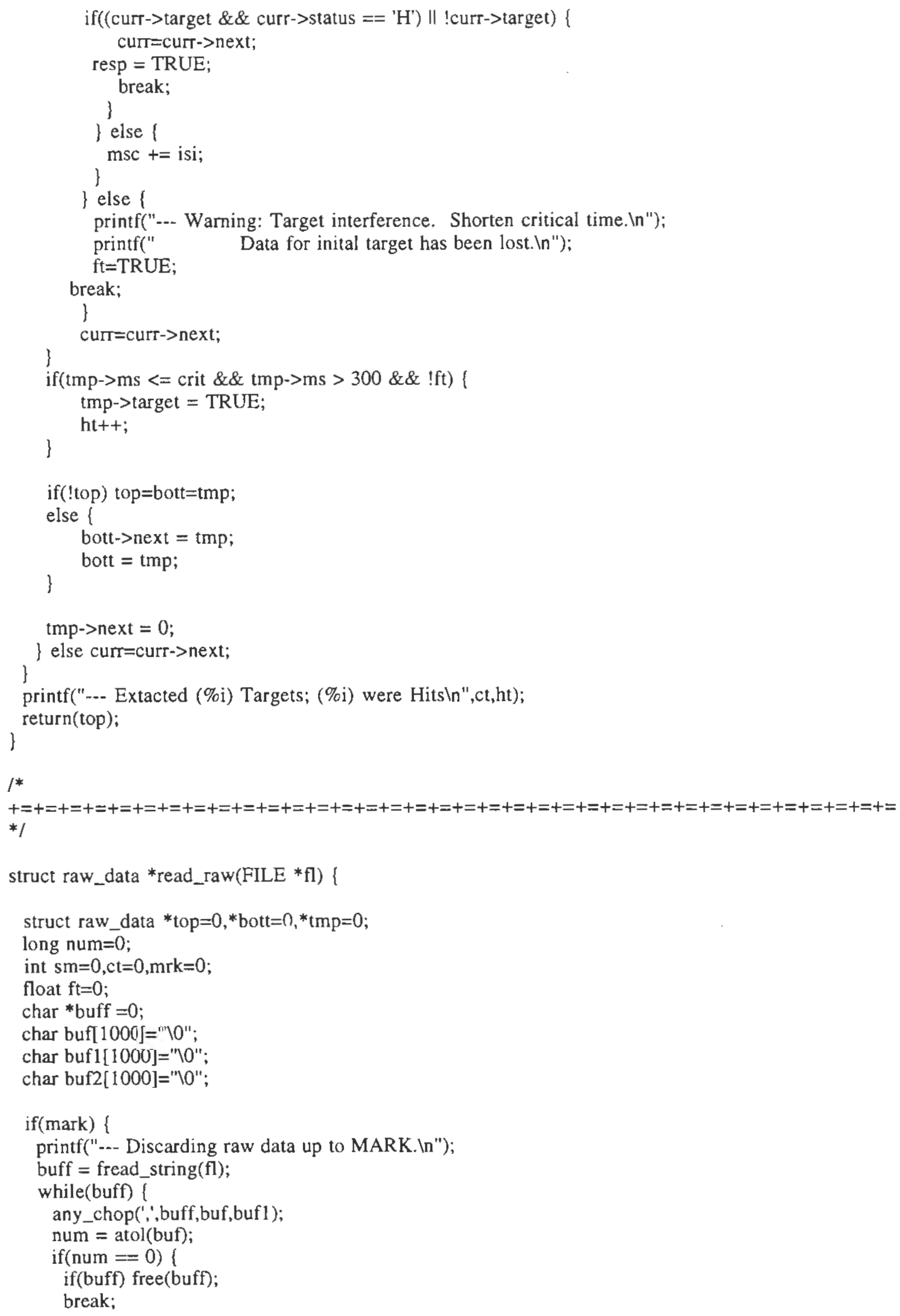




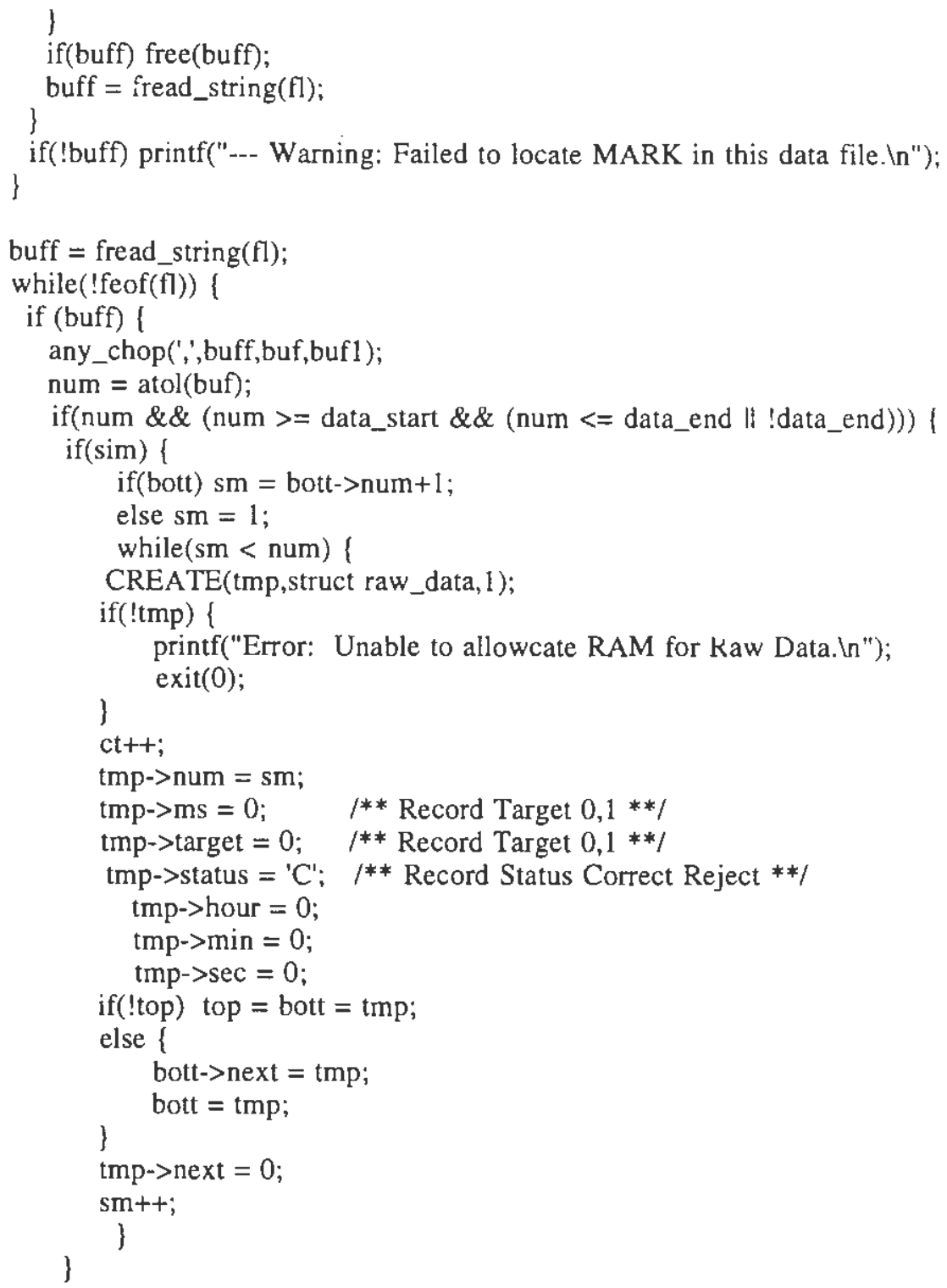




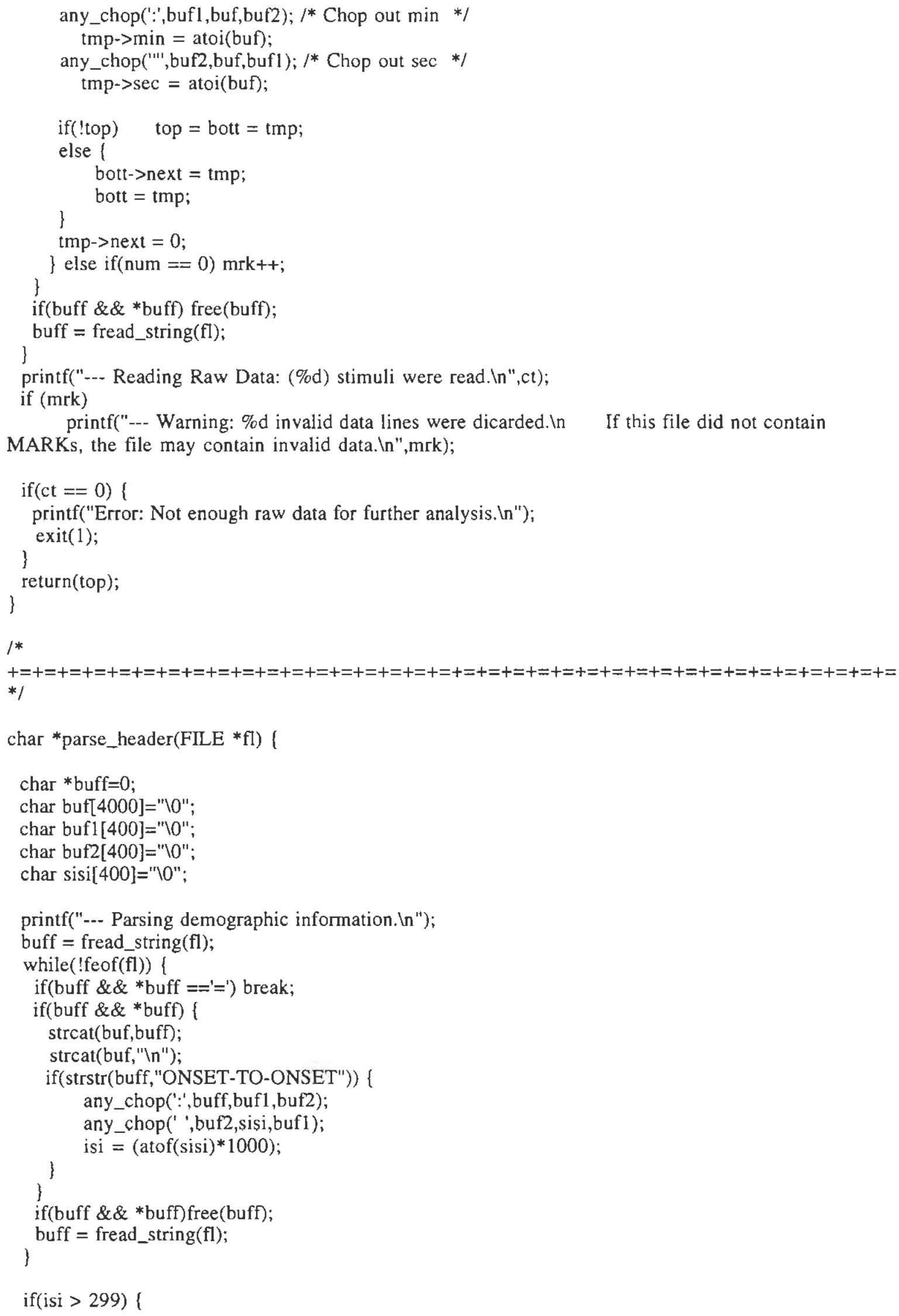


printf("--.- Onset-To-Onset time is (\%d) milliseconds. In",isi);

I else \{

printf("Erтor: Onset-To-Onset time not found or less than 300ms. $\ln ")$; exit( (1);

)

return(strdup(buf));

\}

/*

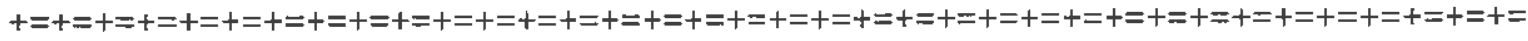
$* 1$

void clrscr(void) \{

int $\mathrm{i}=0$;

for $(\mathrm{i} ; \mathrm{i}<25 ; \mathrm{i}++)$ printf(") $\left.\mathrm{n}^{\prime \prime}\right)$;

\}

/*

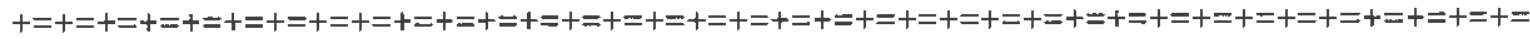
*1 


\section{Appendix B}

\section{Sample of a Simulation Target File}

File: T7.sim

1 and 5 minute frequencies

ONSET-TO-ONSET:.5

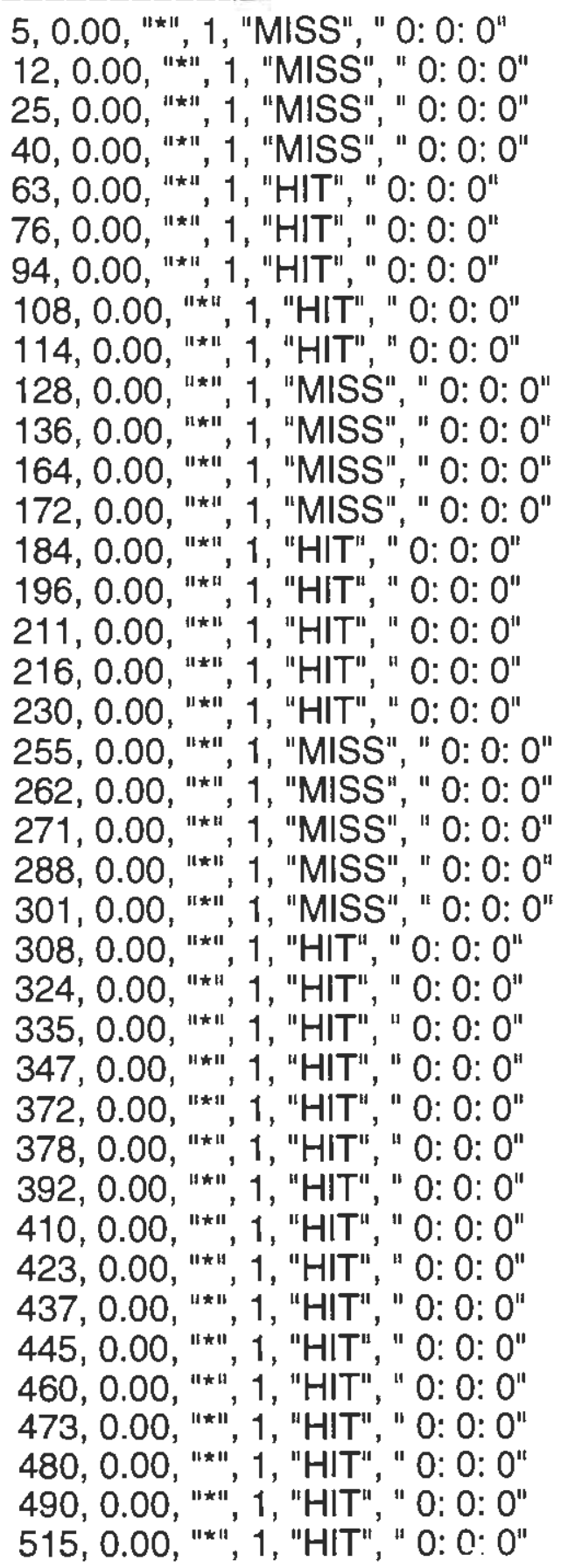


$527,0.00, " * ", 1$, "HIT", " 0: 0:0" $543,0.00$, "**", 1, "HIT", " 0: 0: 0" $553,0.00$, "*" 1, "HIT", " 0: 0: 0" 560, 0.00, "*", 1, "HIT", " 0: 0: 0" 580, 0.00, "*", 1, "HIT", " 0: 0: 0" $593,0.00$, "*", 1, "HIT", " 0: 0: 0" $598,0.00$, "*u, 1, "HIT", " 0: 0: 0" 624, 0.00, "*", 1, "MISS", " 0: 0: 0" 636, 0.00, "*", 1, "MISS", " 0: 0: 0" 649, 0.00, "**", 1, "MISS", " 0: 0: 0" 656, 0.00, "*", 1, "MISS", " 0: 0: 0" $660,0.00, "$ "*", 1, "HIT", " 0: 0: 0" 664, 0.00, "*", 1, "HIT", " 0: 0: 0" 671, 0.00, "*", 1, "HIT", " 0: 0: 0" 684, 0.00, "**", 1, "HIT", " 0: 0: 0" 699, 0.00, "*", 1, "HIT", " 0: 0: 0" 722, 0.00, "**", 1, "MISS", " 0: 0: 0" 735, 0.00, "*", 1, "MISS", " 0: 0: 0" 753, 0.00, "*"1, 1, "MISS", " 0: 0: 0" 767, 0.00, "*", 1, "HIT", " 0: 0: 0" $773,0.00, "$ " 1 , "HIT", " $0: 0: 0$ " 787, 0.00, "*", 1, "HIT", " 0: 0: 0" 795, 0.00, "**" 1, "HIT", " 0: 0: 0" $823,0.00, " * * ", 1$, "HIT", " 0: 0: 0" 831, 0.00, "*", 1, "MISS", " 0: 0: 0" 843, 0.00, "*", 1, "MISS", " 0: 0: 0" $855,0.00$, "*" 1 , "MISS", " 0: 0: 0" $870,0.00$, "*" 1 , "MISS", " 0: 0: 0" 875, 0.00, "*", 1, "HIT", " 0: 0: 0" 889, 0.00, "*", 1, "HIT", " 0: 0: 0" 914, 0.00, "*", 1, "HIT", " 0: 0: 0" 921, 0.00, "*ะ", 1, "HIT", " 0: 0: 0" 930, 0.00, "*", 1, "HIT", " 0: 0: 0" 947, 0.00, "*1", 1, "HIT", " 0: 0: 0" 960, 0.00, "*" 1, "HIT", " 0: 0: 0 " 967, 0.00, "*", 1, "HIT", " 0: 0: $0^{\text {" }}$ 983, 0.00, "*", 1, "HIT", " 0: 0: 0" 994, 0.00, "*", 1, "HIT", " 0: 0: 0" 1006, 0.00, "*", 1, "HIT", " 0: 0: 0" 1031, 0.00, "*" , 1, "HIT", " 0: 0: 0" 1037, 0.00, "*", 1, "HIT", " 0: 0: 0" 1051, 0.00, "**", 1, "HIT", " 0: 0: 0" 1069, 0.00, "*", 1, "HIT", " 0: 0: 0" 1082, 0.00, "*" , 1, "HIT", " 0: 0: 0" 1096, 0.00, "*", 1, "HIT", " 0: 0: 0" 1104, 0.00, "*", 1, "HIT", " 0: 0: 0" 1119, 0.00, "*", 1, "HIT", " 0: 0: 0" 1132, 0.00, "*", 1, "HIT", " 0: 0: 0" 1139, 0.00, "**", 1, "HIT", " 0: 0: 0" 
$1149,0.00$, "*", 1, "HIT", " 0:0:0" 1174, 0.00, "*", 1, "HIT", " 0: 0: 0" $1186,0.00$, "*", 1, "HIT", " 0: 0: 0" 1202, 0.00, "*", 1, "MISS", " 0: 0: 0" 1212, 0.00, "**1, "MISS", " 0: 0: 0" $1219,0.00$, "*1", 1, "MISS", " 0: 0: 0" 1239, 0.00, "*", 1, "MISS", " 0: 0: 0" 1252, 0.00, "*" 1 , "MISS", " 0: 0: $0^{\prime \prime}$ 1257, 0.00, "*", 1, "HIT", " 0: 0: 0" 1283, 0.00, "*", 1, "HIT", " 0: 0: 0" 1295, 0.00, "**", 1, "HIT", " 0: 0: 0" $1308,0.00$, "*", 1, "HIT", " 0: 0: 0" 1315, 0.00, "*", 1, "HIT", " 0: 0: 0" 1319, 0.00, "*", 1, "HIT", " 0: 0: 0" 1323, 0.00, "*", 1, "MISS", " 0: 0: 0" 1330, 0.00, "*", 1, "MISS", " 0: 0: 0" 1343, 0.00, "*", 1, "MISS", " 0: 0: 0" 1358, 0.00, "*", 1, "MISS", " 0: 0: 0" 1381, 0.00, "*", 1, "MISS", " 0: 0: 0" 1394, 0.00, "*", 1, "HIT", " 0: 0: 0" 1412, 0.00, "*", 1, "HIT", " 0: 0: 0" 1426, 0.00, "*", 1, "HIT", " 0: 0: 0" 1432, 0.00, "*", 1, "HIT", " 0: 0: 0" 1446, 0.00, "*" , 1, "MISS", " 0: 0: 0" 1454, 0.00, "*" , 1, "MISS", " 0: 0: 0" 1482, 0.00, "*", 1, "MISS", " 0: 0: 0" 1490, 0.00, "*»"1, "MISS", " 0: 0: 0" 1502, 0.00, "*", 1, "HIT", " 0: 0: 0" 1514, 0.00, "*", 1, "HIT", " 0: 0: 0" 1529, 0.00, "*", 1, "HIT", " 0: 0: 0" 1534, 0.00, "**", 1, "HIT", " 0: 0: 0" 1548, 0.00, "*", 1, "HIT", " 0: 0: 0" 1573, 0.00, "*", 1, "HIT", " 0: 0: 0" 1580, 0.00, "*", 1, "HIT", " 0: 0: 0" 1589, 0.00, "*", 1, "HIT", " 0: 0: 0" $1606,0.00$, "*", 1, "HIT", " 0: 0: 0" 1619, 0.00, "*", 1, "HIT", " 0: 0: 0" 1626, 0.00, "*", 1, "HIT", " 0: 0: 0" 1642, 0.00, "*", 1, "HIT", " 0: 0: 0" 1653, 0.00, "*", 1, "HIT", " 0: 0: 0" 1665, 0.00, "*", 1, "HIT", " 0: 0: 0" 1690, 0.00, "**1", 1, "HIT", " 0: 0: 0" 1696, 0.00, "*", 1, "HIT", " 0: 0: 0" 1710, 0.00, "*", 1, "HIT", " 0: 0: 0 " 1728, 0.00, "*", 1, "HIT", " 0: 0: 0" 1741, 0.00, "*a, 1, "HIT", " 0: 0: 0" 1755, 0.00, "*", 1, "HIT", " 0: 0: 0" 1763, 0.00, "*", 1, "HIT", " 0: 0: 0" $1778,0.00$, "**", 1, "HIT", " 0: 0: 0" 
1791, 0.00, "*", 1, "HIT", " 0: 0: 0" 1798, 0.00, "*" 1 , "HIT", " 0: 0: 0" 1808, 0.00, "**", 1, "MISS", " 0: 0: 0" 1833, 0.00, "*", 1, "MISS", " 0: 0: 0" 1845, 0.00, "*", 1, "MISS", " 0: 0: 0" 1861, 0.00, "*", 1, "MISS", " 0: 0: 0" 1871, 0.00, "*", 1, "HIT", " 0: 0: 0" 1878, 0.00, "**", 1, "HIT", " 0: 0: 0" 1898, 0.00, "*" , 1, "HIT", " 0: 0: 0" 1911, 0.00, "*", 1, "HIT", " 0: 0: 0" 1916, 0.00, "*", 1, "HIT", " 0: 0: 0" 1942, 0.00, "*", 1, "MISS", " 0: 0: 0" 1954, 0.00, "**", 1, "MISS", " 0: 0: 0" 1967, 0.00, "*", 1, "MISS", " 0: 0: 0" 1974, 0.00, "*", 1, "MISS", " 0: 0: 0" 1978, 0.00, "*", 1, "HIT", " 0: 0: 0" 1982, 0.00, "*", 1, "HIT", " 0:0: 0" 1989, 0.00, "*", 1, "HIT", " 0: 0: 0" 2002, 0.00, "*", 1, "HIT", " 0: 0: 0" 2017, 0.00, "**1, 1, "HIT", " 0: 0: 0" 2040, 0.00, "**", 1, "MISS", " 0: 0: 0" 2053, 0.00, "*", 1, "MISS", " 0: 0: 0" 2071, 0.00, "*", 1, "MISS", " 0: 0: 0" 2085, 0.00, "*", 1, "MISS", " 0: 0: 0" 2091, 0.00, "**", 1, "MISS", " 0: 0: 0" 2105, 0.00, "*", 1, "HIT", " 0: 0: 0" 2113, 0.00, "*", 1, "HIT", " $0: 0: 0$ " 2141, 0.00, "*" , 1, "HIT", " 0: 0: 0" 2149, 0.00, "*", 1, "HIT", " 0: 0: 0" 2161, 0.00, "*" , 1, "HIT", " 0: 0: 0" 2173, 0.00, "*", 1, "HIT", " 0: 0: 0" 2188, 0.00, "*", 1, "HIT", " 0: 0: 0" 2193, 0.00, "*", 1, "HIT", " 0: 0: 0" 2207, 0.00, "*", 1, "HIT", " 0: 0: 0" 2232, 0.00, "**", 1, "HIT", " 0: 0: 0" 2239, 0.00, "**", 1, "HIT", " 0: 0: $0^{\prime \prime}$ 2248, 0.00, "** , 1, "HIT", " 0: 0: 0" 2265, 0.00, "*", 1, "HIT", " 0: 0: 0" 2278, 0.00, "*", 1, "HIT", " 0: 0: 0" 2285, 0.00, "*", 1, "HIT", " 0: 0: 0" 2301, 0.00, "*", 1, "HIT", " 0: 0: $0^{\text {" }}$ 2312, 0.00, "*", 1, "HIT", " 0: 0: 0" 2324, 0.00, "*", 1, "HIT", " 0: 0: 0" 2349, 0.00, "*", 1, "HIT", " 0: 0: 0" 2355, 0.00, "*", 1, "HIT", " 0: 0: 0" 2369, 0.00, "*", 1, "HIT", " 0: 0: 0" 2387, 0.00, "*", 1, "HIT", " 0: 0: 0" 2400, 0.00, "*", 1, "MISS", " 0:0:0" 


\section{Appendix C}

\section{Experiment 1, Part 1 ANOVA Tables}

$\ldots$.... ONEWAY ....

Variable \# of Hit Runs (Bin 1)

By Variable Group

Analysis of Variance

\begin{tabular}{llllll}
\hline Source & D.F. & $\begin{array}{l}\text { Sum of } \\
\text { Squares }\end{array}$ & $\begin{array}{l}\text { Mean } \\
\text { Squares }\end{array}$ & $\begin{array}{l}F \\
\text { Ratio }\end{array}$ & $\begin{array}{l}F \\
\text { Prob. }\end{array}$ \\
\hline & & & & & \\
Between Groups & 1 & 7.2946 & 7.2946 & .7129 & .3987 \\
Within Groups & 1010 & 10334.2657 & 10.2319 & & \\
Total & 1011 & 10341.5603 & & & \\
\hline
\end{tabular}

$\ldots$. . . ONEWAY .....

Variable Length of Hit Run (Bin 1)

By Variable Group

Analysis of Variance

\begin{tabular}{llllll} 
& & & & & \\
Source & D.F. & Sum of & Mean & $\mathrm{F}$ & $\mathrm{F}$ \\
Squares & Squares & Ratio & Prob. \\
\hline Between Groups & 1 & & & & \\
Within Groups & 1010 & 42.1215 & .0417 & 13.3655 & .0003 \\
Total & 1011 & 42.6789 & & & \\
\hline
\end{tabular}


Variable \# of Hit Runs (Bin 2)

By Variable Group

Analysis of Variance

\begin{tabular}{llllll}
\hline & & Sum of & Mean & F & F \\
Source & D.F. & Squares & Squares & Ratio & Prob. \\
\hline & & & & & \\
Between Groups & 1 & .0170 & .0170 & .0028 & .9579 \\
Within Groups & 1009 & 6161.5023 & 6.1065 & & \\
Total & 1010 & 6161.5193 & & & \\
\hline
\end{tabular}

$\ldots$... ONEWAY ....

Variable Length of Hit Run (Bin 2)

By Variable Group

Analysis of Variance

\begin{tabular}{|c|c|c|c|c|c|}
\hline Source & D.F. & $\begin{array}{l}\text { Sum of } \\
\text { Squares }\end{array}$ & $\begin{array}{l}\text { Mean } \\
\text { Squares }\end{array}$ & $\begin{array}{l}\text { F } \\
\text { Ratio }\end{array}$ & $\begin{array}{l}\text { F } \\
\text { Prob. }\end{array}$ \\
\hline $\begin{array}{l}\text { Between Groups } \\
\text { Within Groups }\end{array}$ & $\begin{array}{l}1 \\
1009\end{array}$ & $\begin{array}{l}.0306 \\
121.5521\end{array}$ & $\begin{array}{l}.0306 \\
.1205\end{array}$ & .2540 & .6144 \\
\hline Total & 1010 & 121.5827 & & & \\
\hline
\end{tabular}


Variable \# of Hit Runs (Bin 3)

By Variable Group

Analysis of Variance

\begin{tabular}{llllll}
\hline Source & D.F. & $\begin{array}{l}\text { Sum of } \\
\text { Squares }\end{array}$ & $\begin{array}{l}\text { Mean } \\
\text { Squares }\end{array}$ & $\begin{array}{l}F \\
\text { Ratio }\end{array}$ & $\begin{array}{l}F \\
\text { Prob. }\end{array}$ \\
\hline & & & & & \\
Between Groups & 1 & 1.8214 & 1.8214 & .5400 & .4626 \\
Within Groups & 1007 & 3396.9199 & 3.3733 & & \\
Total & 1008 & 3398.7413 & & & \\
\hline
\end{tabular}

ONEWAY ....

Variable Length of Hit Run (Bin 3)

By Variable Group

Analysis of Variance

\begin{tabular}{llllll}
\hline Source & D.F. & $\begin{array}{l}\text { Sum of } \\
\text { Squares }\end{array}$ & $\begin{array}{l}\text { Mean } \\
\text { Squares }\end{array}$ & $\begin{array}{l}F \\
\text { Ratio }\end{array}$ & Prob. \\
\hline Between Groups & 1 & & & & \\
Within Groups & 1007 & .4400 & .4400 & 1.6054 & .2054 \\
Total & 1008 & 275.9882 & .2741 & & \\
\hline
\end{tabular}


Variable \# of Hit Runs (Bin 4)

By Variable Group

Analysis of Variance

\begin{tabular}{llllll} 
& & & & \\
Source & D.F. & Squares & $\begin{array}{l}\text { Mean } \\
\text { Squares }\end{array}$ & Ratio & Prob. \\
\hline Between Groups & 1 & & & & \\
Within Groups & 1006 & 28.2601 & 28.2601 & 13.1452 & .0003 \\
Total & 1007 & 2162.7310 & 2.1498 & & \\
\hline
\end{tabular}

ONEWAY ....

Variable Length of Hit Run (Bin 4)

By Variable Group

Analysis of Variance

\begin{tabular}{llllll}
\hline Source & D.F. & $\begin{array}{l}\text { Sum of } \\
\text { Squares }\end{array}$ & $\begin{array}{l}\text { Mean } \\
\text { Squares }\end{array}$ & $\begin{array}{l}F \\
\text { Ratio }\end{array}$ & $\begin{array}{l}F \\
\text { Prob. }\end{array}$ \\
\hline & & & & & \\
Between Groups & 1 & 61.0589 & 61.0589 & 81.8754 & .0000 \\
Within Groups & 1006 & 750.2284 & .7458 & & \\
Total & 1007 & 811.2873 & & & \\
\hline
\end{tabular}




\section{Appendix D}

\section{Experiment 1, Part $1 \mathrm{Chi}$ Squares}

Exp 1 Part 1 Bin 1

\begin{tabular}{l|cc|cc|c}
\multicolumn{1}{c|}{} & \multicolumn{3}{c|}{$\begin{array}{c}\text { Human } \\
\text { Subj. }\end{array}$} & \multicolumn{3}{c}{$\begin{array}{c}\text { Modeled } \\
\text { Subj. }\end{array}$} \\
\cline { 2 - 6 } Freq. Obs. & $\mathrm{f}_{0}=$ & 24 & $\mathrm{f}_{\mathrm{o}}=$ & 21 & \multirow{2}{*}{45} \\
& $\mathrm{f}_{\mathrm{e}}=$ & 22.5 & $\mathrm{f}_{\mathrm{e}}=$ & 22.5 & \\
\hline No Freq. Obs. & $\mathrm{f}_{\mathrm{o}}=$ & 16 & $\mathrm{f}_{\mathrm{o}}=$ & 19 & 35 \\
& $\mathrm{f}_{\mathrm{e}}=$ & 17.5 & $\mathrm{f}_{\mathrm{e}}=$ & 17.5 & \\
\cline { 2 - 6 } & & 40 & & 40 & 80
\end{tabular}

$$
\begin{aligned}
X^{2}{ }_{\text {obt }} & =0.457 \quad X^{2}{ }_{\text {cril }}=3.84 \\
d f & =1
\end{aligned}
$$

\begin{tabular}{|c|c|c|c|c|c|}
\hline \multirow[b]{2}{*}{ Freq. Obs. } & \multicolumn{2}{|c|}{$\begin{array}{l}\text { Human } \\
\text { Subj. }\end{array}$} & \multicolumn{2}{|c|}{$\begin{array}{l}\text { Modeled } \\
\text { Subj. }\end{array}$} & \multirow[b]{2}{*}{53} \\
\hline & $\begin{array}{l}f_{0}= \\
f_{e}=\end{array}$ & $\begin{array}{c}30 \\
26.5\end{array}$ & $\begin{array}{l}f_{0}= \\
f_{e}=\end{array}$ & $\begin{array}{c}23 \\
26.5 \\
\end{array}$ & \\
\hline \multirow[t]{3}{*}{ No Freq. Obs. } & $\begin{array}{l}f_{0}= \\
f_{e}=\end{array}$ & $\begin{array}{c}10 \\
13.5\end{array}$ & $\begin{array}{l}f_{0}= \\
f_{e}=\end{array}$ & $\begin{array}{c}17 \\
13.5\end{array}$ & 27 \\
\hline & & 40 & & 40 & 80 \\
\hline & \multicolumn{2}{|c|}{$\begin{aligned} X^{2}{ }_{o b t} & =2.739 \\
d f & =1\end{aligned}$} & \multicolumn{2}{|c|}{$X^{2}{ }_{\text {crim }}=3.84$} & \\
\hline
\end{tabular}

Exp 1 Part 1 Bin 2 
Exp 1 Part 1 Bin 3

\begin{tabular}{|c|c|c|c|c|c|}
\hline \multirow[b]{2}{*}{ Freq. Obs. } & \multicolumn{2}{|c|}{$\begin{array}{l}\text { Human } \\
\text { Subj. }\end{array}$} & \multicolumn{2}{|c|}{$\begin{array}{c}\text { Modeled } \\
\text { Subj. }\end{array}$} & \multirow[b]{2}{*}{45} \\
\hline & $\begin{array}{l}f_{0}= \\
f_{e}=\end{array}$ & $\begin{array}{c}25 \\
22.5 \\
\end{array}$ & $\begin{array}{l}f_{0}= \\
f_{e}=\end{array}$ & $\begin{array}{c}20 \\
22.5\end{array}$ & \\
\hline \multirow[t]{3}{*}{ No Freq. Obs. } & $\begin{array}{l}f_{0}= \\
f_{e}=\end{array}$ & $\begin{array}{c}15 \\
17.5\end{array}$ & $\begin{array}{l}f_{0}= \\
f_{e}=\end{array}$ & $\begin{array}{c}20 \\
17.5\end{array}$ & 35 \\
\hline & & 40 & & 40 & 80 \\
\hline & \multicolumn{2}{|c|}{$\begin{aligned} X^{2}{ }_{o b t} & =1.270 \\
d f & =1\end{aligned}$} & \multicolumn{2}{|c|}{$X^{2}{ }_{\text {cril }}=3.84$} & \\
\hline
\end{tabular}

Exp 1 Part 1 Bin 4

\begin{tabular}{l|cc|cc|c}
\multicolumn{1}{c|}{} & \multicolumn{3}{c|}{$\begin{array}{c}\text { Human } \\
\text { Subj. }\end{array}$} & \multicolumn{3}{c}{$\begin{array}{c}\text { Modeled } \\
\text { Subj. }\end{array}$} \\
\cline { 2 - 5 } Freq. Obs. & $\mathrm{f}_{\mathrm{o}}=$ & 12 & $\mathrm{f}_{\mathrm{o}}=$ & 5 & 17 \\
& $\mathrm{f}_{\mathrm{e}}=$ & 8.5 & $\mathrm{f}_{\mathrm{e}}=$ & 8.5 & \\
\hline No Freq. Obs. & $\mathrm{f}_{\mathrm{o}}=$ & 28 & $\mathrm{f}_{\mathrm{o}}=$ & 35 & 63 \\
$\mathrm{f}_{\mathrm{e}}=$ & 31.5 & $\mathrm{f}_{\mathrm{e}}=$ & 31.5 & \\
\cline { 2 - 6 } & & 40 & & 40 & 80
\end{tabular}

$$
\begin{aligned}
X^{2}{ }_{o b t} & =3.660 \quad X^{2}{ }_{c n t}=3.84 \\
d f & =1
\end{aligned}
$$




\section{Appendix E}

\section{Experiment 1, Part 2 ANOVA Tables}

$\ldots$. . . ONEWAY ....

Variable Average Hit Time

By Variable Groups

Analysis of Variance

\begin{tabular}{llllll}
\hline Source & D.F. & $\begin{array}{l}\text { Sum of } \\
\text { Squares }\end{array}$ & $\begin{array}{l}\text { Mean } \\
\text { Squares }\end{array}$ & $\begin{array}{l}\text { F } \\
\text { Ratio }\end{array}$ & $\begin{array}{l}\text { F } \\
\text { Prob. }\end{array}$ \\
\hline & & & & & \\
Between Groups & 1 & 4894.3326 & 4894.3326 & .7094 & .4068 \\
$\begin{array}{l}\text { Within Groups } \\
\text { Total }\end{array}$ & 28 & 193167.1163 & 6898.8256 & & \\
\hline
\end{tabular}

ONEWAY ....

Variable Number of False Alarms

By Variable Groups

Analysis of Variance

\begin{tabular}{llllll}
\hline Source & D.F. & $\begin{array}{l}\text { Sum of } \\
\text { Squares }\end{array}$ & $\begin{array}{l}\text { Mean } \\
\text { Squares }\end{array}$ & $\begin{array}{l}\mathrm{F} \\
\text { Ratio }\end{array}$ & $\begin{array}{l}\text { F } \\
\text { Prob. }\end{array}$ \\
\hline Between Groups & 1 & 885.6333 & 885.6333 & 7.6552 & \\
Within Groups & 28 & 3239.3333 & 115.6905 & & \\
Total & 29 & 4124.9667 & & & \\
\hline
\end{tabular}


Variable Ratio Sort/Long Inter-Target-Interval Hits following Hits By Variable Groups

Analysis of Variance

\begin{tabular}{llllll}
\hline Source & D.F. & $\begin{array}{l}\text { Sum of } \\
\text { Squares }\end{array}$ & $\begin{array}{l}\text { Mean } \\
\text { Squares }\end{array}$ & $\begin{array}{l}\mathrm{F} \\
\text { Ratio }\end{array}$ & $\begin{array}{l}\mathrm{F} \\
\text { Prob. }\end{array}$ \\
\hline & & & & & \\
Between Groups & 1 & .0145 & .0145 & .5219 & .4760 \\
Within Groups & 28 & .7790 & .0278 & & \\
Total & 29 & .7935 & & & \\
\hline
\end{tabular}

-... . ONEWAY ....

Variable Ratio Sort/Long Inter-Target-Interval Misses following Hits By Variable Groups

Analysis of Variance

\begin{tabular}{llllll}
\hline Source & D.F. & $\begin{array}{l}\text { Sum of } \\
\text { Squares }\end{array}$ & $\begin{array}{l}\text { Mean } \\
\text { Squares }\end{array}$ & $\begin{array}{l}\text { F } \\
\text { Ratio }\end{array}$ & $\begin{array}{l}\text { F } \\
\text { Prob. }\end{array}$ \\
\hline & & & & & \\
Between Groups & 1 & 3.6450 & 3.6450 & 2.2102 & .1483 \\
Within Groups & 28 & 46.1763 & 1.6492 & & \\
Total & 29 & 49.8212 & & & \\
\hline
\end{tabular}




\section{Appendix F}
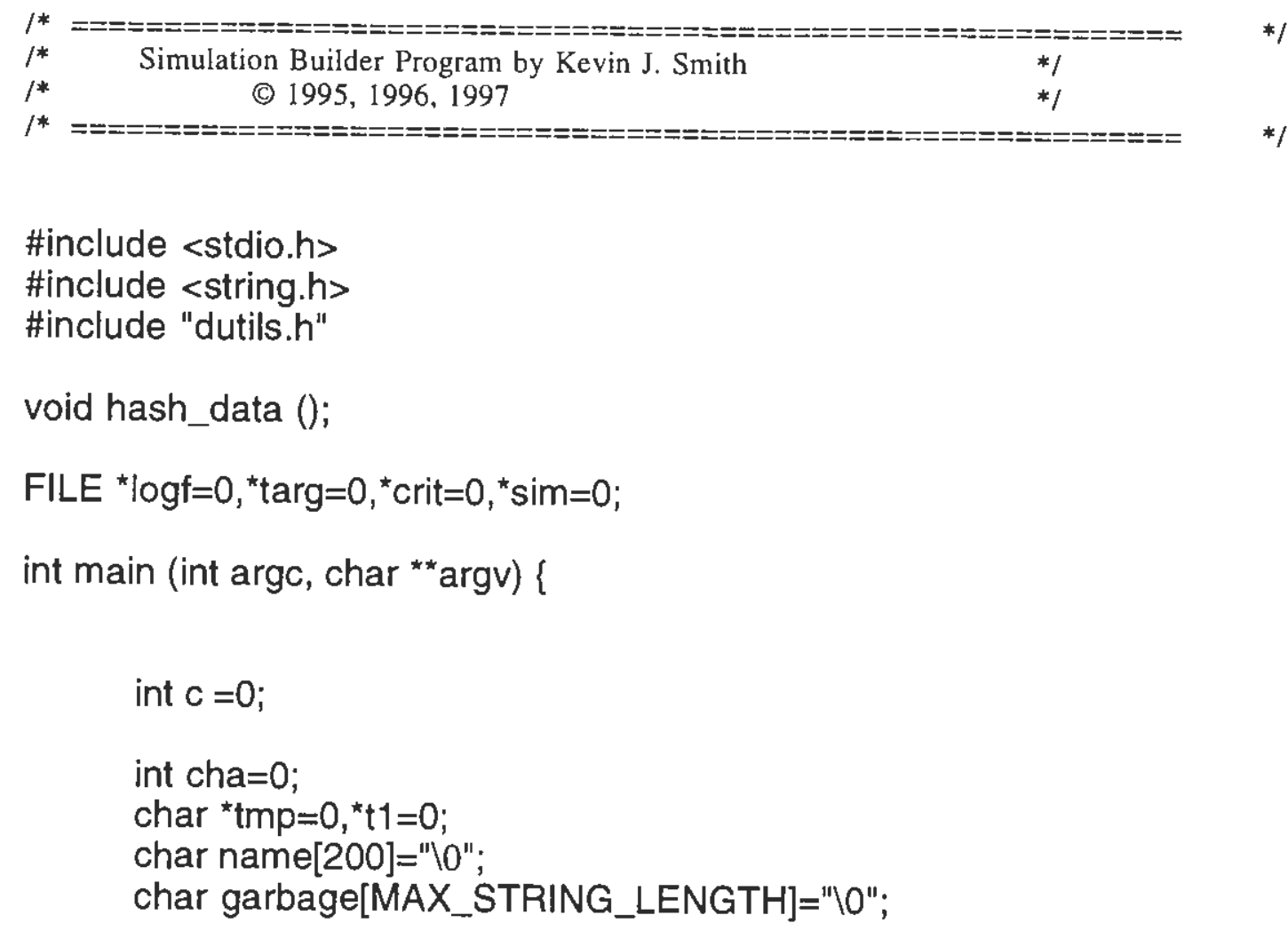

printf("Usage: $\operatorname{sim}$ (Target file) (Criteria File) (Simulation Filename) $\backslash n ")$; exit(0); \}

\#ifdef DEBUG1

\#endif

$$
\text { printf("ARGV[1]=\%sin",(argv[1])); }
$$

printf("sim : Target time file not found. (\%s)\n",(argv[1]));

exit(0);

\}

\#ifdef DEBUG1

\#endif

$$
\text { printf("ARGV[2]=\%sln",(argv[2])); }
$$

printf("sim : Criteria file not found. (\%s)/n",(argv[2]));

exit(0); 


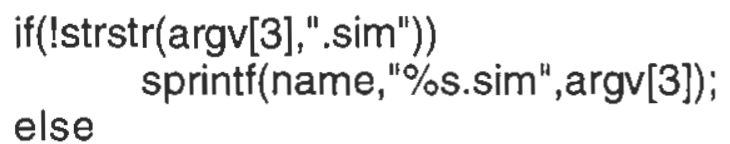

strcpy(name, argv[3]);

if (!(sim=fopen(name, "w+"))) \{

\#ifdef DEBUG1

\#endif

$$
\text { printf("NAME=\%sln",name); }
$$

printf("sim : Unable to create .sim output file. (\%s) \n",name);

exit(0);

\}

hash_data();

fclose(targ);

fclose(crit);

fclose(sim);

printf("Program exited normally. In");

\}

void hash_data () \{

int num $=0, \operatorname{comp}=0$,tnum $=0$;

char temp[200]=" 10 ";

char ${ }^{*} \mathrm{t}=0$;

\#ifdef DEBUG1

\# endif

$$
\text { printt("-- HASH_DATA --In"); }
$$

$\mathrm{t}=$ fread_string(crit);

comp = atoi $(t)$;

if(t) free $(t)$;

$t=0$;

printf("First crit=\%dln",comp);

while(comp II !feof(targ)) \{

printf("Looking for \#\%d... In",comp);

if(feof(targ)) break;

while(num != comp \&\& !feof(targ)) \{

$\mathrm{t}=$ fread_string(targ);

if(t) tnum = atoi(t);

if $\left(t \& \&{ }^{*} t\right)$ free $(t) ;$

$\mathrm{t}=0$;

if(tnum) num++;

if (num != comp \&\& tnum)

fprintf(sim," \%d, 0.00, l"k।", 1, I"MISSI", I" 0: 0: 01"In",tnum);

printf(" \%d (\%d) -- MISSIn",num,tnum); 


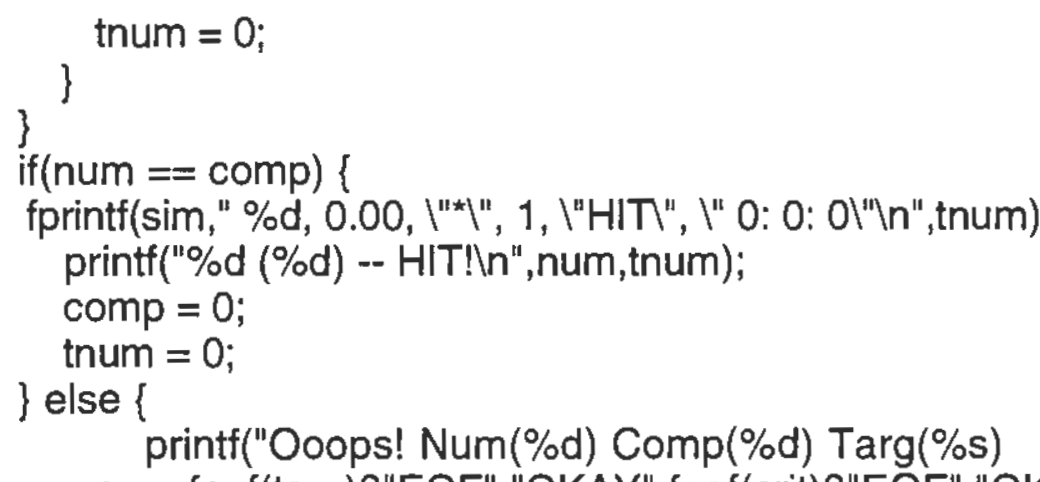

Crit(\%s)'n",num,comp,feof(targ)?"EOF":"OKAY",feof(crit)?"EOF":"OKAY"); break;

\}

$\mathrm{t}=$ fread_string(crit);

if $(\mathrm{t}) \operatorname{comp}=$ atoi $(\mathrm{t})$;

if $\left(t \& \&{ }^{*} t\right)$ free $(t)$;

$\mathrm{t}=0$;

printf("Next crit=\%d\n",comp); 


\section{Appendix G}

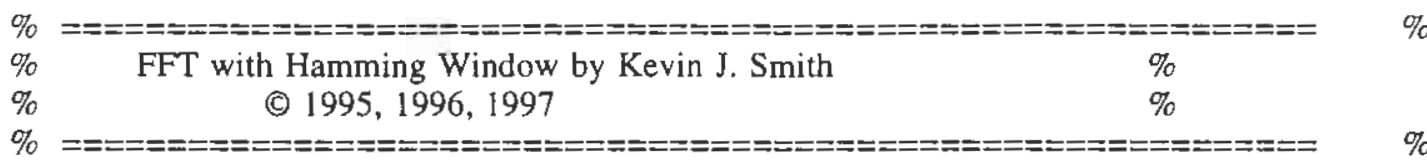

function [power] =hamfft(a, sis, nm, crit)

$A=a^{\prime}$;

$a=a^{\prime} ;$

$x=(1$ :sis:sis*length $(A))$;

$p=\operatorname{polyfit}(x, A, 1)$;

$f=\operatorname{polyval}(p, x)$;

figure(1);

$\mathrm{a}=\mathrm{a}-\mathrm{f} ;$

ham=hamming(length(a));

$\mathrm{a}=$ ham'. ${ }^{*}$;

$Y=f f t(a, 1024)$;

$Y(1)=\square ;$

$\mathrm{n}=$ length $(\mathrm{Y})$;

$\mathrm{z}=$ linspace $(0,0, \mathrm{n})$;

power $=\operatorname{abs}(Y(1: n / 2)) . \wedge 2$;

nyquist $=1 / 2$;

freq $=(1: n / 2) /(n / 2)^{\star}$ nyquist;

$\%$ figure(3);

clf;

plot(freq,power,'-w');

xlabel(['Cycles per ',num2str(sis),' Second Step']);

ylabel('Power');

title(['FFT with Hamming Window -- Subject ',nm]);

hold:

if(crit $==0$ )

if $(\max (\operatorname{power}(1: n / 2))<20)$

crit $=\max (\operatorname{power}(1: n / 2)) / 2$;

else

if $(\max (\operatorname{power}(1: n / 2))>1000)$

crit $=\max (\operatorname{power}(1: n / 2)) / 2$;

crit $=$ crit $-($ crit/ $/ 3)$;

else

$$
\text { crit }=20 \text {; }
$$




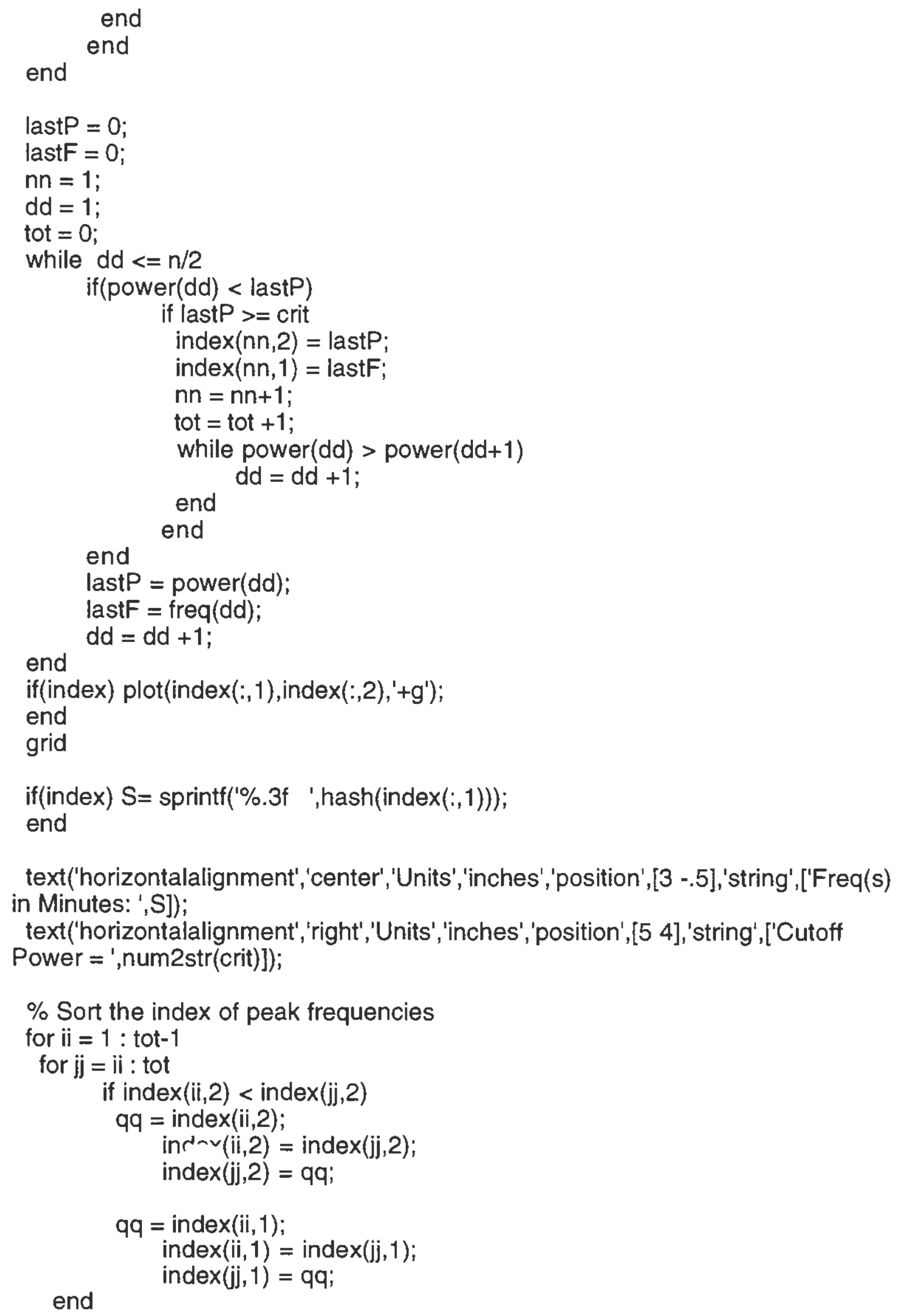




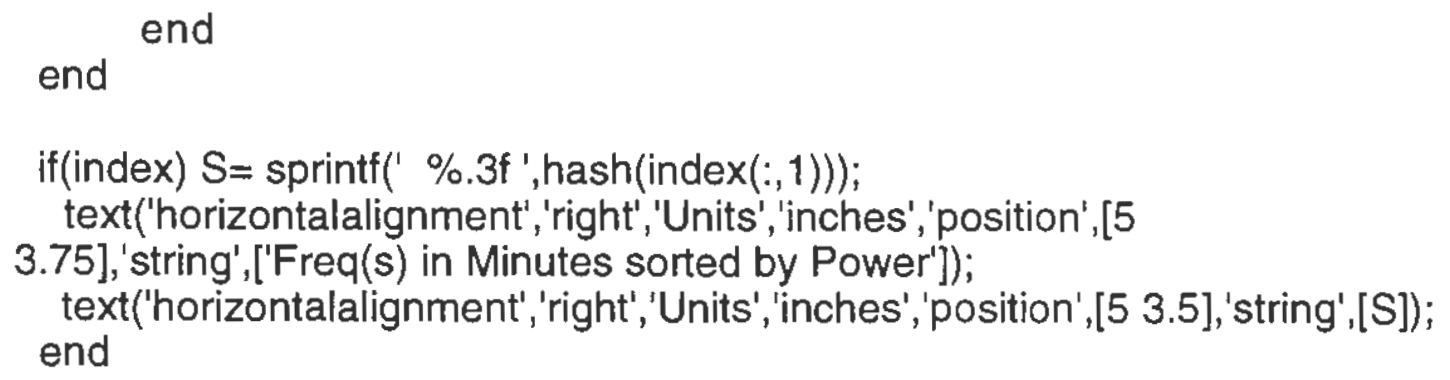




\section{Appendix $\mathrm{H}$}

\section{MAW and FFT Power Spectrum Pair Examples}
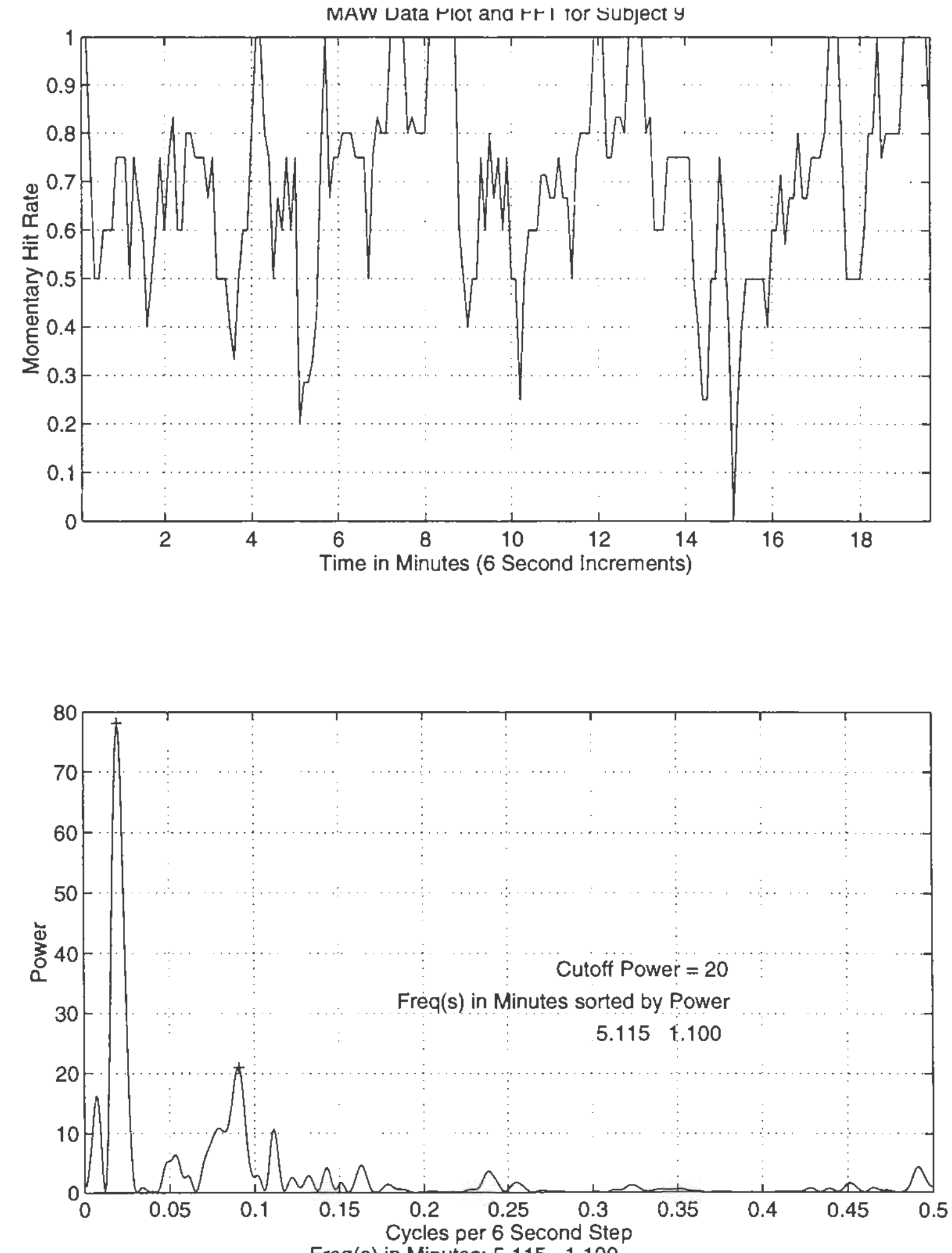

Freq(s) in Minutes: 5.1151 .100 
MAW vata r'lot and rトI ror suoject 31
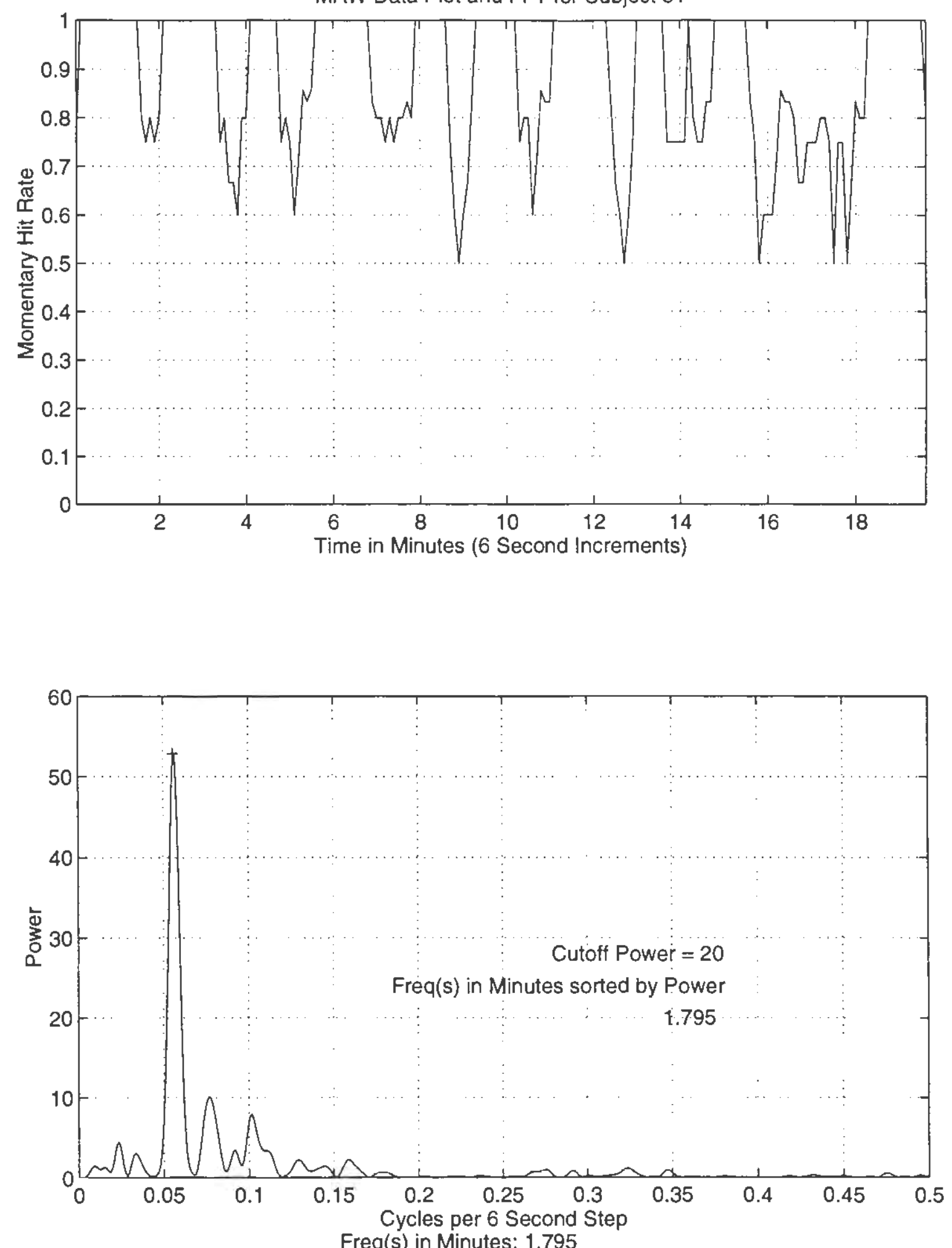

Freq(s) in Minutes: 1.795 

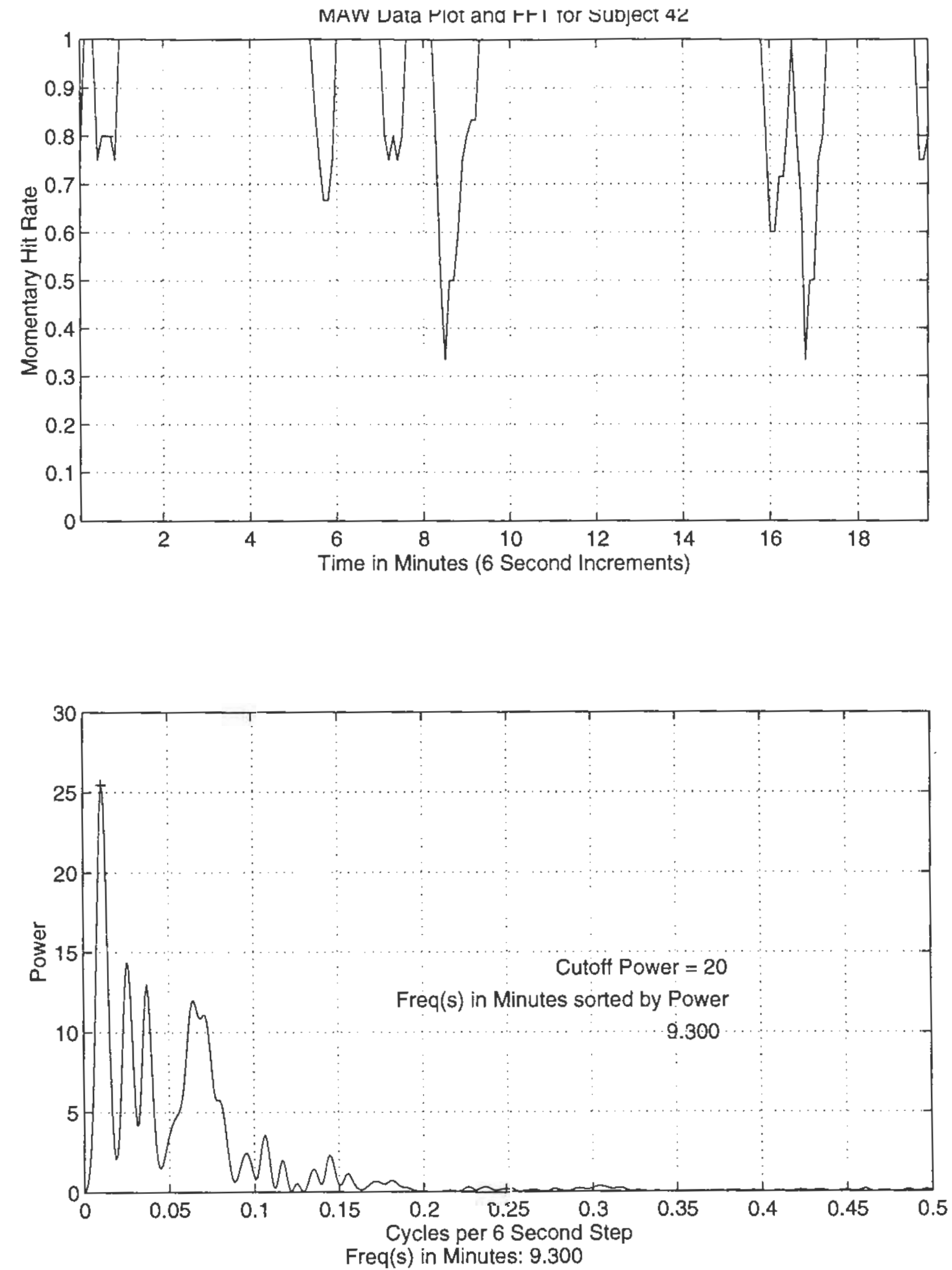
MAW Uata rlot and rr I for subject bb
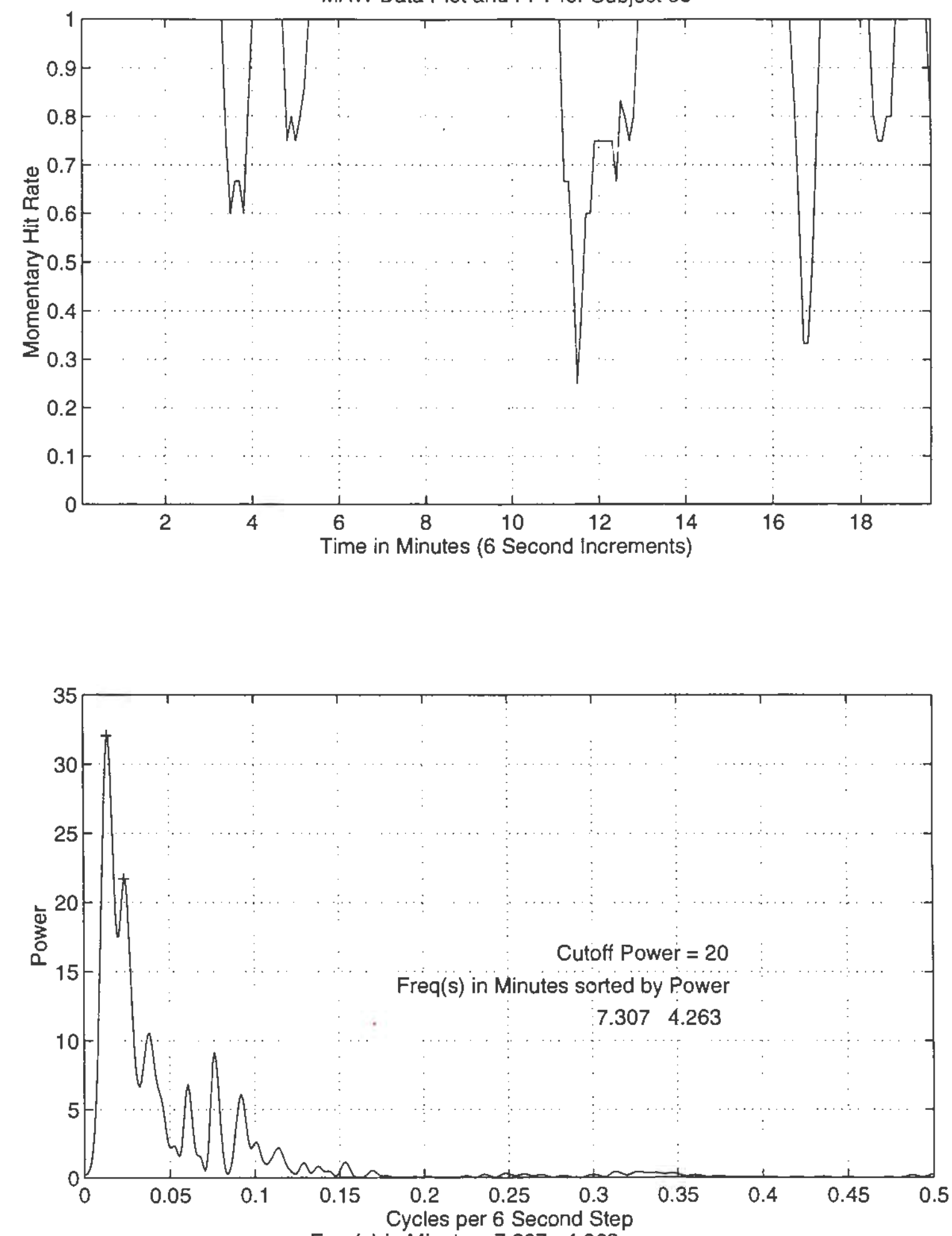

Freq(s) in Minutes: $7.307 \quad 4.263$ 


\section{Appendix I}

\section{Experiment 2 Part 3 Chi Squares}

Exp 2 Part 3 Bin 1

\begin{tabular}{|c|c|c|c|c|c|}
\hline \multirow[b]{2}{*}{ Freq. Obs. } & \multicolumn{2}{|r|}{$\begin{array}{c}\text { Human } \\
\text { Subj. }\end{array}$} & \multicolumn{2}{|c|}{$\begin{array}{c}\text { Modeled } \\
\text { Subj. }\end{array}$} & \multirow[b]{2}{*}{45} \\
\hline & $\begin{array}{l}f_{0}= \\
f_{e}=\end{array}$ & $\begin{array}{c}24 \\
22.5\end{array}$ & $\begin{array}{l}f_{0}= \\
f_{e}=\end{array}$ & $\begin{array}{c}21 \\
22.5\end{array}$ & \\
\hline \multirow[t]{3}{*}{ No Freq. Obs. } & $\begin{array}{l}f_{0}= \\
f_{e}=\end{array}$ & $\begin{array}{c}16 \\
17.5\end{array}$ & $\begin{array}{l}f_{0}= \\
f_{e}=\end{array}$ & $\begin{array}{c}19 \\
17.5\end{array}$ & 35 \\
\hline & & 40 & & 40 & 80 \\
\hline & \multicolumn{2}{|c|}{$\begin{aligned} X^{2}{ }_{o b t} & =0.457 \\
d f & =1\end{aligned}$} & \multicolumn{2}{|c|}{$X^{2}{ }_{\text {crit }}=3.84$} & \\
\hline
\end{tabular}

Exp 2 Part 3 Bin 2

\begin{tabular}{l|ccc|cc|c}
\multicolumn{1}{c|}{} & \multicolumn{3}{c|}{$\begin{array}{c}\text { Human } \\
\text { Subj. }\end{array}$} & \multicolumn{2}{c}{$\begin{array}{c}\text { Modeled } \\
\text { Subj. }\end{array}$} \\
\cline { 2 - 6 } Freq. Obs. & $f_{0}=$ & 30 & $f_{0}=$ & 23 & 53 \\
& $f_{e}=$ & 26.5 & $f_{e}=$ & 26.5 & \\
\hline No Freq. Obs. & $f_{0}=$ & 10 & $f_{0}=$ & 17 & 27 \\
$f_{e}=$ & 13.5 & $f_{e}=$ & 13.5 & \\
\cline { 2 - 6 } & & 40 & & 40 & 80
\end{tabular}

$$
\begin{aligned}
X^{2}{ }_{a b t} & =2.739 \quad X^{2}{ }_{c r i l}=3.84 \\
d f & =1
\end{aligned}
$$


Exp 2 Part 3 Bin 3

\begin{tabular}{|c|c|c|c|c|c|}
\hline \multirow[b]{2}{*}{ Freq. Obs. } & \multicolumn{2}{|r|}{$\begin{array}{c}\text { Human } \\
\text { Subj. }\end{array}$} & \multicolumn{2}{|c|}{$\begin{array}{c}\text { Modeled } \\
\text { Subj. }\end{array}$} & \multirow[b]{2}{*}{45} \\
\hline & $\begin{array}{l}f_{0}= \\
f_{e}=\end{array}$ & $\begin{array}{c}25 \\
22.5\end{array}$ & $\begin{array}{l}f_{0}= \\
f_{e}=\end{array}$ & $\begin{array}{c}20 \\
22.5 \\
\end{array}$ & \\
\hline \multirow[t]{3}{*}{ No Freq. Obs. } & $\begin{array}{l}f_{0}= \\
f_{e}=\end{array}$ & $\begin{array}{c}15 \\
17.5\end{array}$ & $\begin{array}{l}f_{0}= \\
f_{e}=\end{array}$ & $\begin{array}{c}20 \\
17.5\end{array}$ & 35 \\
\hline & & 40 & & 40 & 80 \\
\hline & \multicolumn{2}{|c|}{$\begin{aligned} X^{2}{ }_{o b l} & =1.270 \\
d f & =1\end{aligned}$} & \multicolumn{2}{|c|}{$X^{2}{ }_{\text {cril }}=3.84$} & \\
\hline
\end{tabular}

Exp 2 Part 3 Bin 4

\begin{tabular}{|c|c|c|c|c|c|}
\hline \multirow[b]{2}{*}{ Freq. Obs. } & & $\begin{array}{l}\text { Human } \\
\text { Subj. }\end{array}$ & & $\begin{array}{l}\text { Modelec } \\
\text { Subj. }\end{array}$ & \multirow[b]{2}{*}{17} \\
\hline & $\begin{array}{l}f_{0}= \\
f_{e}=\end{array}$ & $\begin{array}{l}12 \\
8.5 \\
\end{array}$ & $\begin{array}{l}f_{0}= \\
f_{e}=\end{array}$ & $\begin{array}{c}5 \\
8.5 \\
\end{array}$ & \\
\hline \multirow[t]{3}{*}{ No Freq. Obs. } & $\begin{array}{l}f_{0}= \\
f_{e}=\end{array}$ & $\begin{array}{c}28 \\
31.5\end{array}$ & $\begin{array}{l}f_{0}= \\
f_{\theta}=\end{array}$ & $\begin{array}{c}35 \\
31.5\end{array}$ & 63 \\
\hline & & 40 & & 40 & 80 \\
\hline & \multicolumn{2}{|c|}{$\begin{aligned} X^{2}{ }_{o b t} & =3.660 \\
d f & =1\end{aligned}$} & \multicolumn{2}{|c|}{$X^{2}{ }_{c r i t}=3.84$} & \\
\hline
\end{tabular}




\section{Appendix J}

\section{Experiment 2 Part 4 Chi Squares}

Exp. 2 Part $4 \quad$ Bin 1

\begin{tabular}{|c|c|c|c|c|c|}
\hline & G1 & $\mathrm{G} 2$ & G3 & G4 & \\
\hline Observed & $\begin{array}{lc}f o= & 9 \\
f e= & 7.2\end{array}$ & $\begin{array}{l}f o=6 \\
f e=6.6\end{array}$ & $\begin{array}{lc}f o= & 6 \\
f e= & 5.4\end{array}$ & $\begin{array}{lc}f o= & 3 \\
f e= & 4.8\end{array}$ & 24 \\
\hline \multirow[t]{2}{*}{ Not Observed } & $\begin{array}{lcc}f o= & 3 \\
f e & =4.8\end{array}$ & $\begin{array}{l}f o=5 \\
f e=4.4\end{array}$ & $\begin{array}{l}f_{0}=3 \\
f e=3.6\end{array}$ & $\begin{array}{l}f o= \\
f e=\end{array}$ & 16 \\
\hline & 12 & 11 & 9 & 8 & 40 \\
\hline
\end{tabular}

$X^{2}$ obt $=1.261 \quad X^{2} \mathrm{crit}=7.81$

$d f=3$

Exp. 2 Part $4 \quad$ Bin 2

\begin{tabular}{|c|c|c|c|c|c|c|}
\hline \multirow[b]{2}{*}{ Observed } & Gi & $\mathrm{G} 2$ & G3 & & G4 & \multirow[b]{2}{*}{30} \\
\hline & $\begin{array}{l}f o=10 \\
f e=9\end{array}$ & $\begin{array}{l}f o=8 \\
f e=8.25\end{array}$ & $\begin{array}{ll}f o= & 8 \\
f e= & 6.75\end{array}$ & $\begin{array}{l}f o= \\
f e=\end{array}$ & $\begin{array}{l}4 \\
6\end{array}$ & \\
\hline \multirow[t]{2}{*}{ Not Observed } & $\begin{array}{l}\text { fo }= \\
f e=\end{array}$ & $\begin{array}{l}f o=3 \\
f e=2.75\end{array}$ & $\begin{array}{l}f o=1 \\
f e=2.25\end{array}$ & $\begin{array}{l}\text { fo }= \\
f e=\end{array}$ & $\begin{array}{l}4 \\
2\end{array}$ & 10 \\
\hline & 12 & 11 & 9 & & 8 & 40 \\
\hline
\end{tabular}

$\mathrm{X}^{2} \mathrm{obt}=0.475 \quad \mathrm{X}^{2} \mathrm{crit}=7.81$

$\mathrm{df}=3$ 
Exp. 2 Part 4 Bin 3

\begin{tabular}{|c|c|c|c|c|c|c|}
\hline & G1 & $\mathrm{G} 2$ & G3 & & G4 & \\
\hline Observed & $\begin{array}{l}f o=8 \\
f e=7.5\end{array}$ & $\begin{array}{l}f o=7 \\
f e=6.88\end{array}$ & $\begin{array}{lc}f o= & 5 \\
f e= & 5.63\end{array}$ & $\begin{array}{l}f o= \\
f e=\end{array}$ & $\begin{array}{l}5 \\
5\end{array}$ & 25 \\
\hline \multirow[t]{2}{*}{ Not Observed } & $\begin{array}{l}f o=4 \\
f e=4.5\end{array}$ & $\begin{array}{l}f o=4 \\
f e=4.13\end{array}$ & $\begin{array}{l}f o=4 \\
f e=3.375\end{array}$ & $\begin{array}{l}f o= \\
f e=\end{array}$ & $\begin{array}{l}3 \\
3\end{array}$ & 15 \\
\hline & 12 & 11 & 9 & & 8 & 40 \\
\hline
\end{tabular}

$$
\begin{aligned}
x^{2} \text { obt } & =0.095 \quad x^{2} \text { crit }=7.81 \\
d f & =3
\end{aligned}
$$

Exp. 2 Part $4 \quad$ Bin 4

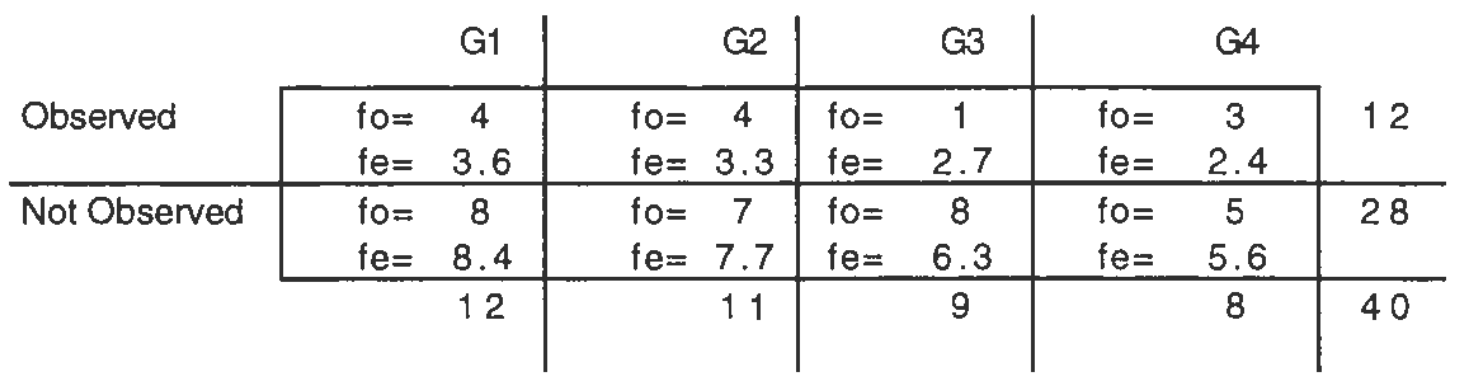

$\mathrm{X}^{2} \mathrm{obt}=0.276 \quad \mathrm{X}^{2} \mathrm{crit}=7.81$

$d f=3$ 


\section{Appendix K}

\section{Experiment 3 Part 1 Chi Squares}

Exp 3 Part 1 Bin 1

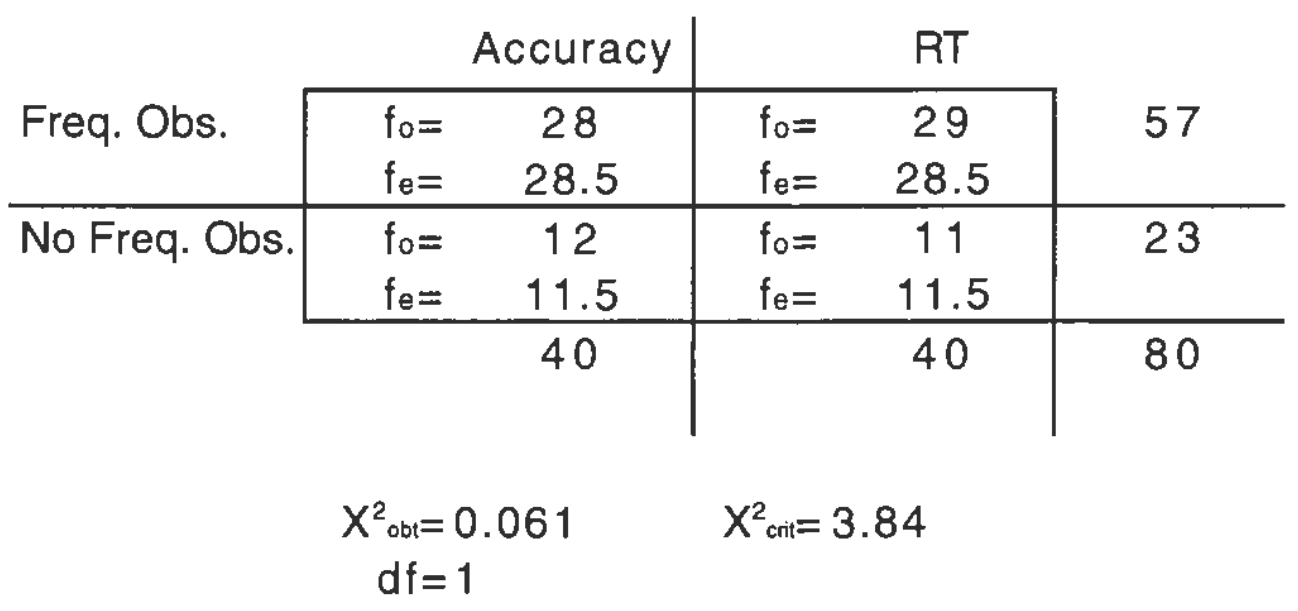

Exp 3 Part 1 Bin 2

\begin{tabular}{l|cc|cc|c}
\multicolumn{1}{c}{} & \multicolumn{3}{c|}{ Accuracy } & \multicolumn{2}{c}{ RT } \\
\cline { 2 - 6 } Freq. Obs. & $\mathrm{f}_{\mathrm{o}}=$ & 34 & $\mathrm{f}_{\mathrm{o}}=$ & 29 & 63 \\
& $\mathrm{f}_{\mathrm{e}}=$ & 31.5 & $\mathrm{f}_{\mathrm{e}}=$ & 31.5 & \\
\hline No Freq. Obs. & $\mathrm{f}_{\mathrm{o}}=$ & 6 & $\mathrm{f}_{\mathrm{o}}=$ & 11 & 17 \\
& $\mathrm{f}_{\mathrm{e}}=$ & 8.5 & $\mathrm{f}_{\mathrm{e}}=$ & 8.5 & \\
\cline { 2 - 6 } & & 40 & & 40 & 80
\end{tabular}

$$
\begin{aligned}
X^{2}{ }_{\text {ob }} & =1.867 \quad X^{2}{ }_{\text {cril }}=3.84 \\
d f & =1
\end{aligned}
$$


Exp 3 Part 1 Bin 3

\begin{tabular}{l|cc|cc|c}
\multicolumn{1}{c}{ Accuracy } & \multicolumn{2}{c}{ RT } \\
\cline { 2 - 5 } Freq. Obs. & $f_{0}=$ & 29 & $f_{0}=$ & 29 & 58 \\
& $f_{e}=$ & 29 & $f_{e}=$ & 29 & \\
\hline No Freq. Obs. & $f_{0}=$ & 11 & $f_{0}=$ & 11 & 22 \\
$f_{e}=$ & 11 & $f_{e}=$ & 11 & \\
\cline { 2 - 5 } & & & & 40 & 80
\end{tabular}

$$
\begin{aligned}
X^{2}{ }^{2 b} & =0.000 \quad X^{2}{ }_{c n t}=3.84 \\
d f & =1
\end{aligned}
$$

Exp 3 Part 1 Bin 4

\begin{tabular}{l|cc|cc|c}
\multicolumn{1}{c|}{} & \multicolumn{2}{c|}{ Accuracy } & \multicolumn{2}{c}{ RT } \\
\cline { 2 - 6 } Freq. Obs. & $f_{0}=$ & 12 & $f_{0}=$ & 23 & 35 \\
& $f_{e}=$ & 17.5 & $f_{e}=$ & 17.5 & \\
\hline No Freq. Obs. & $f_{0}=$ & 28 & $f_{0}=$ & 17 & 45 \\
$f_{e}=$ & 22.5 & $f_{e}=$ & 22.5 & \\
\cline { 2 - 5 } & & 40 & & 40 & 80
\end{tabular}

$$
\begin{aligned}
X^{2}{ }_{o b l} & =6.146 \quad X^{2} \text { crl }=3.84 \\
d f & =1
\end{aligned}
$$




\section{Appendix L}

Accuracy vs. RT FFT Power Spectra 


\section{Human Subject j009, Group 1}

Hit Rate: 0.692

Average Reaction Time: 660.391

Accuracy FFT

Reaction Time FFT
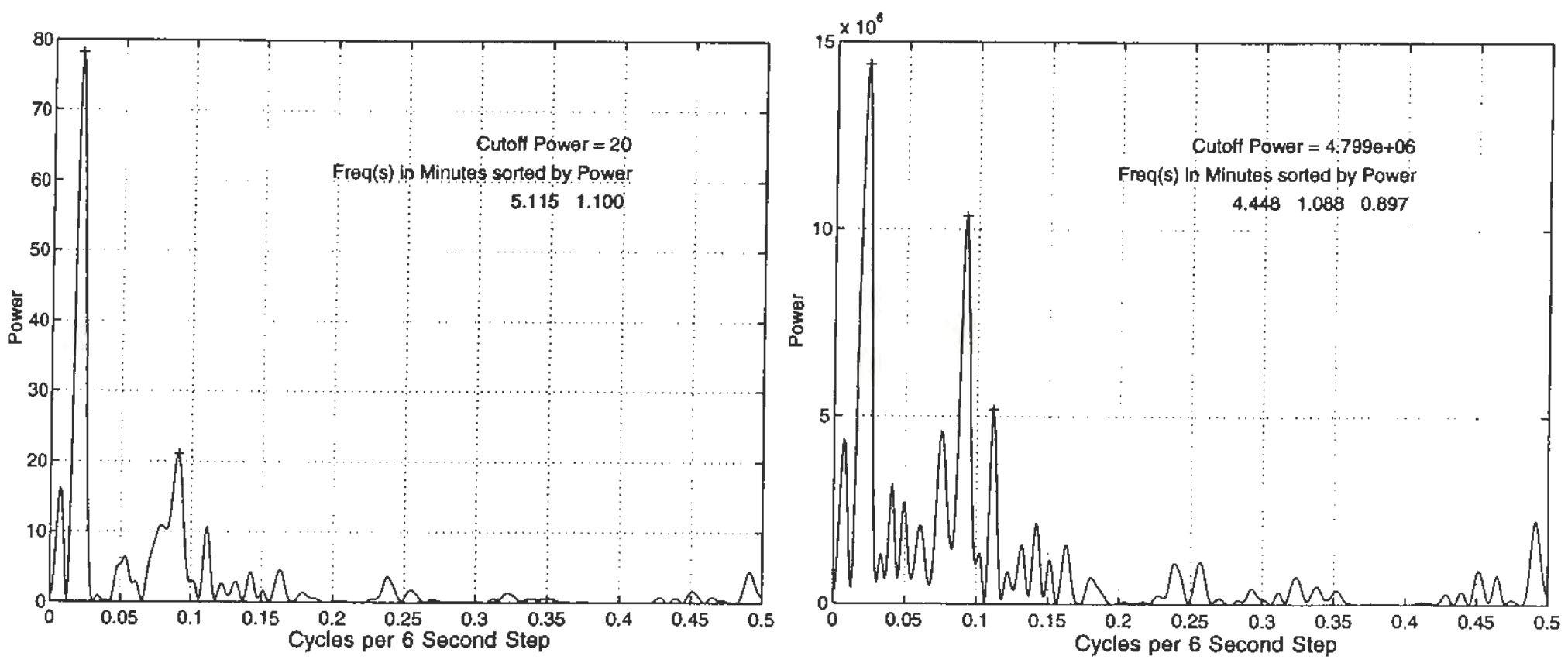


\section{Human Subject j010, Group 1}

Hit Rate: 0.611

Accuracy FFT

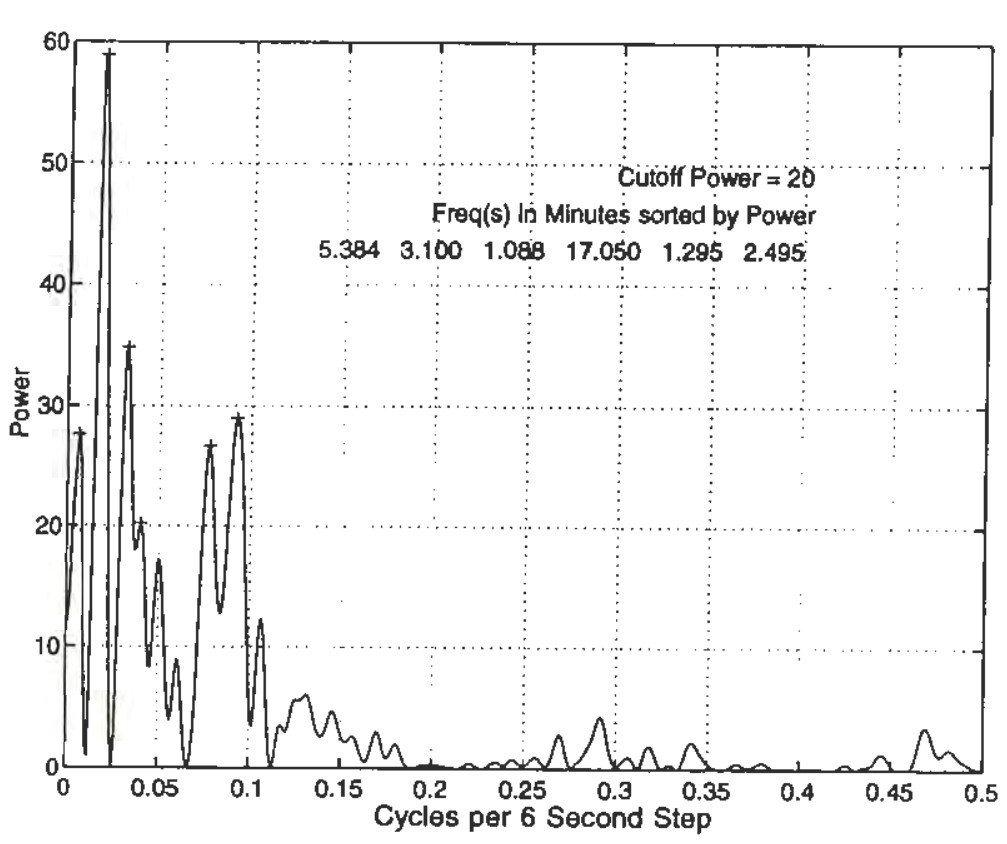

Average Reaction Time: 685.044

Reaction Time FFT

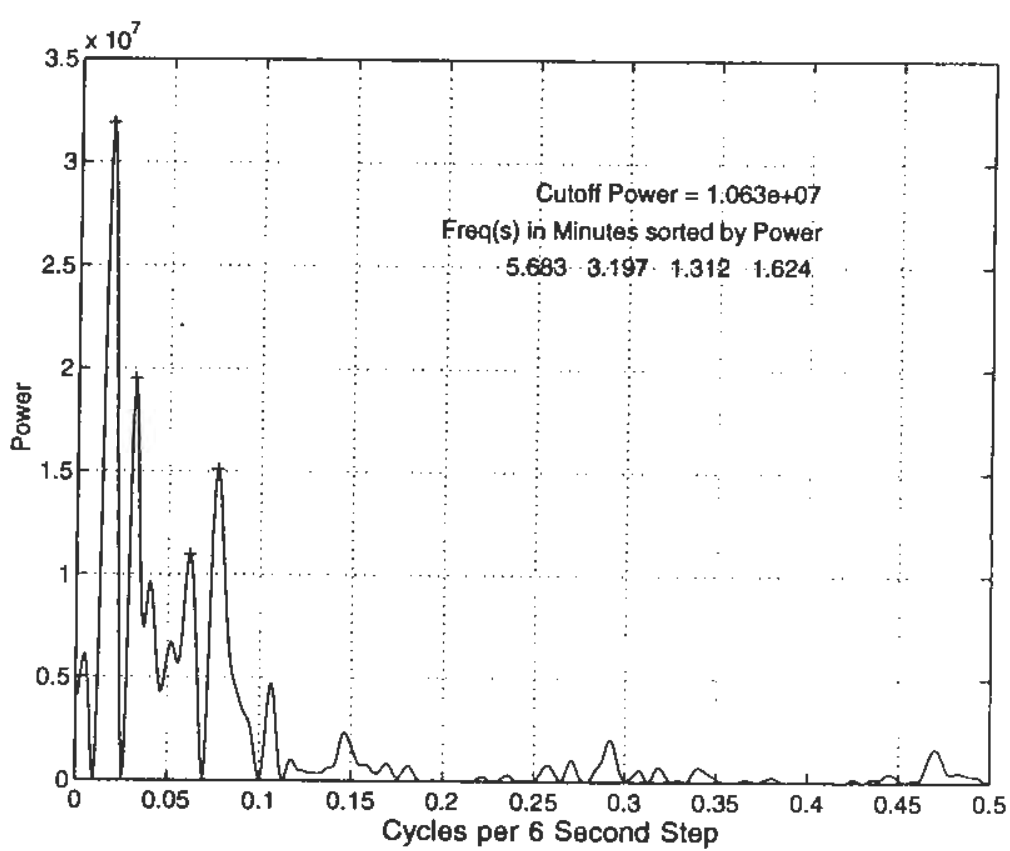




\section{Human Subject j011, Group 1}

Hit Rate: 0.584

\section{Accuracy FFT}

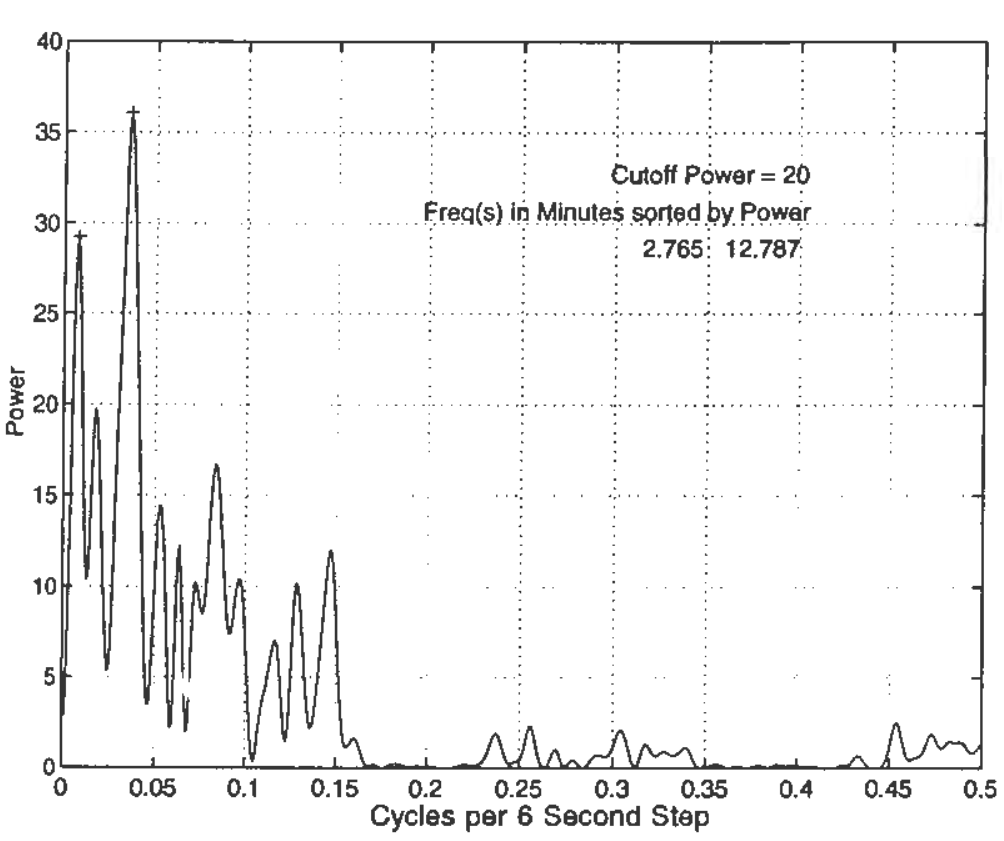

Average Reaction Time: 754.074

Reaction Time FFT

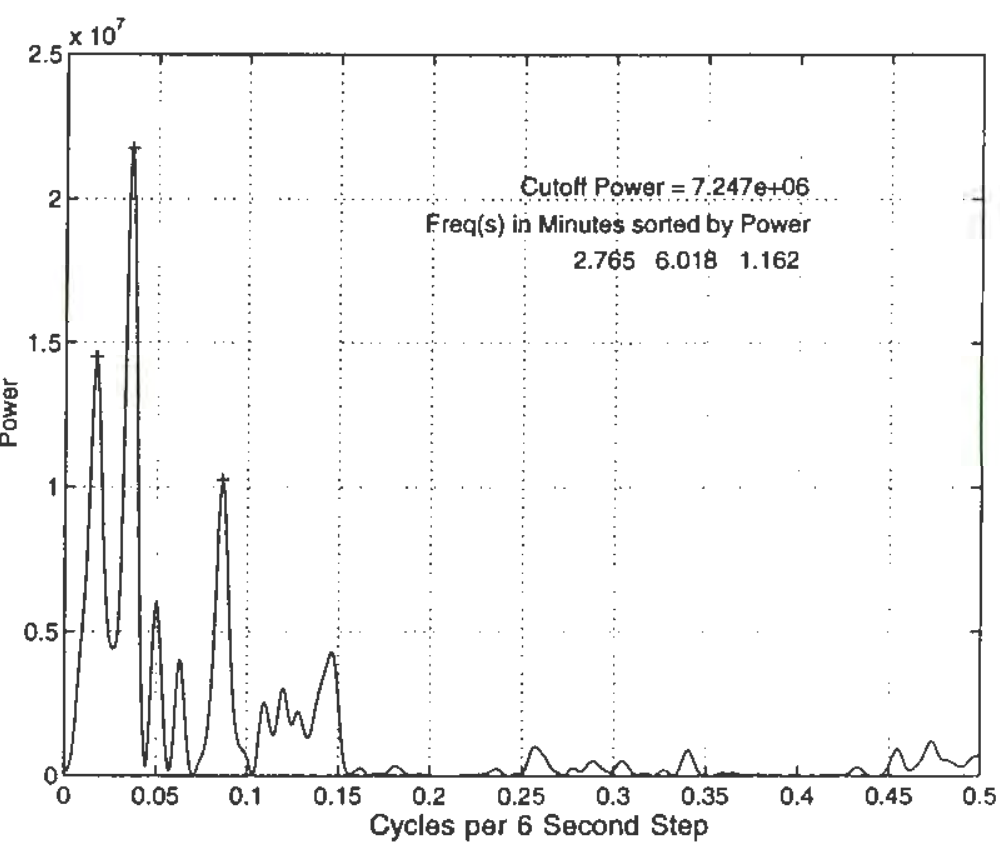




\section{Human Subject j012, Group 1}

Hit Rate: 0.605

\section{Accuracy FFT}

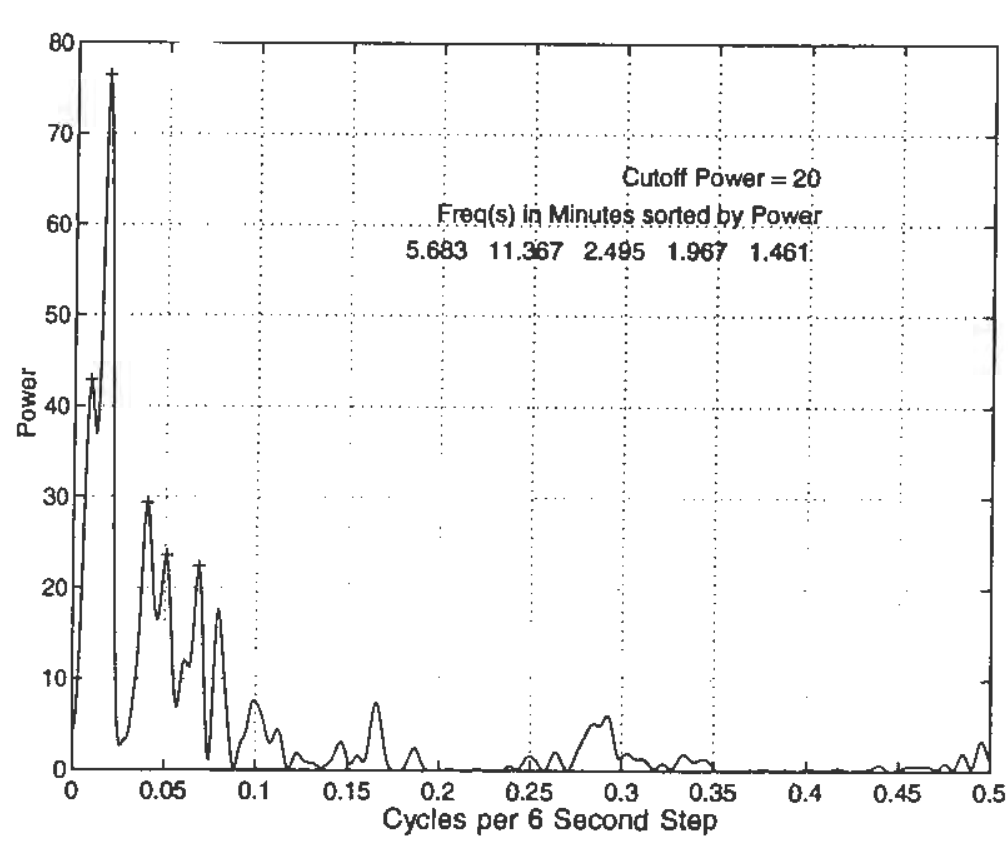

Average Reaction Time: 680.536

\section{Reaction Time FFT}

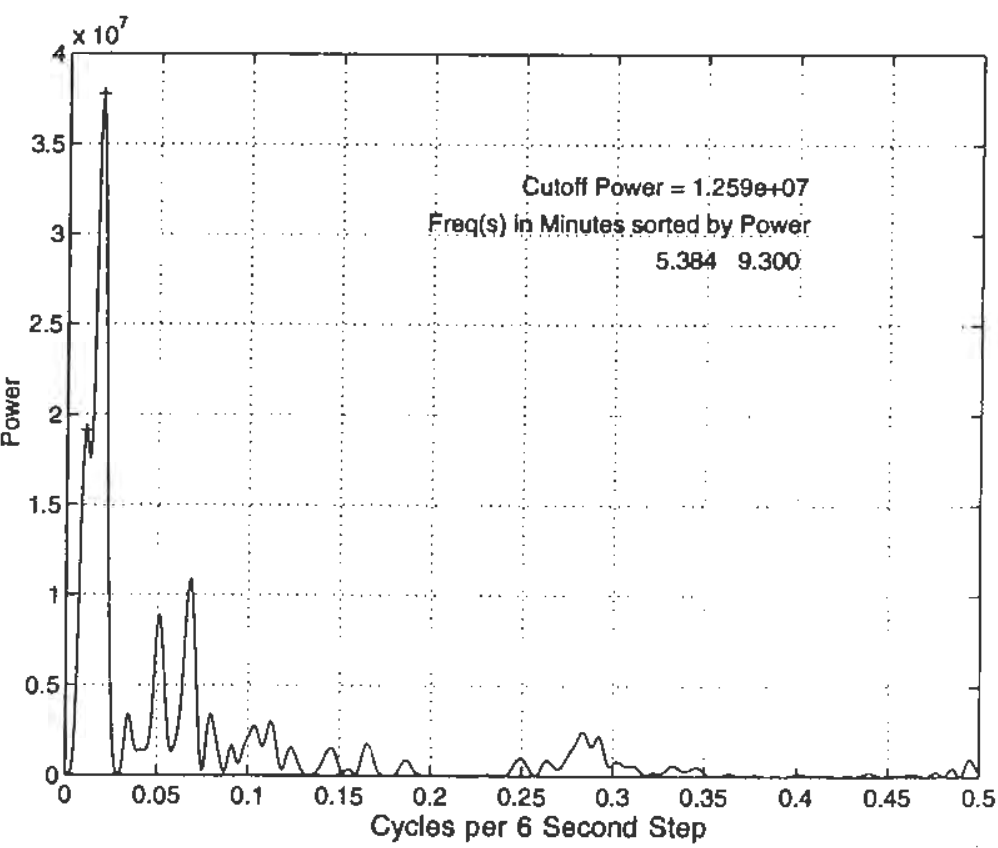




\section{Human Subject j013, Group 1}

Hit Rate: 0.616

Accuracy FFT

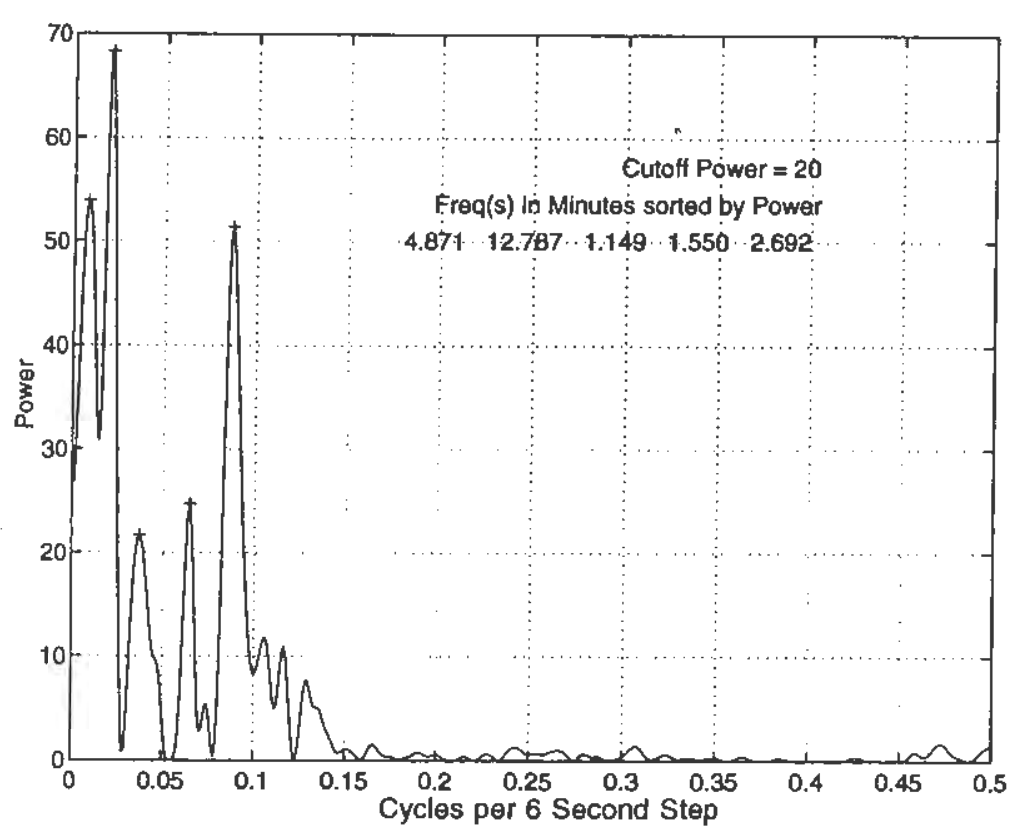

Average Reaction Time: $\mathbf{5 8 5 . 1 7 5}$

Reaction Time FFT

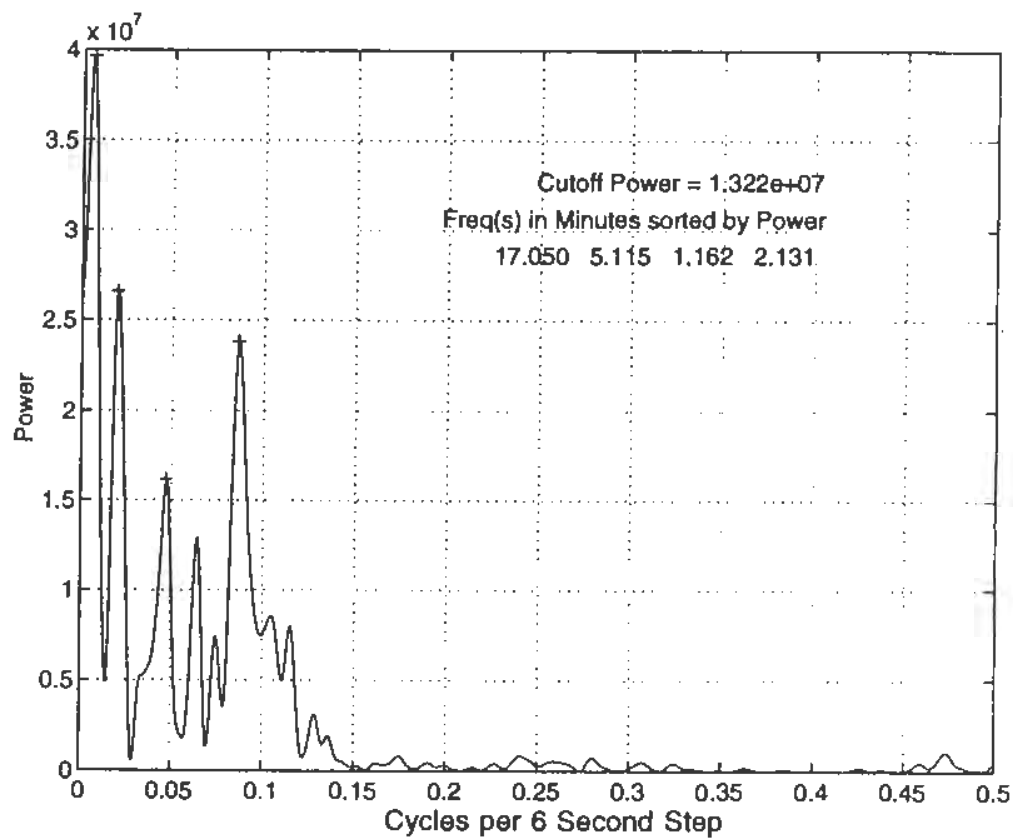




\section{Human Subject j016, Group 1}

Hit Rate: 0.605

Accuracy FFT

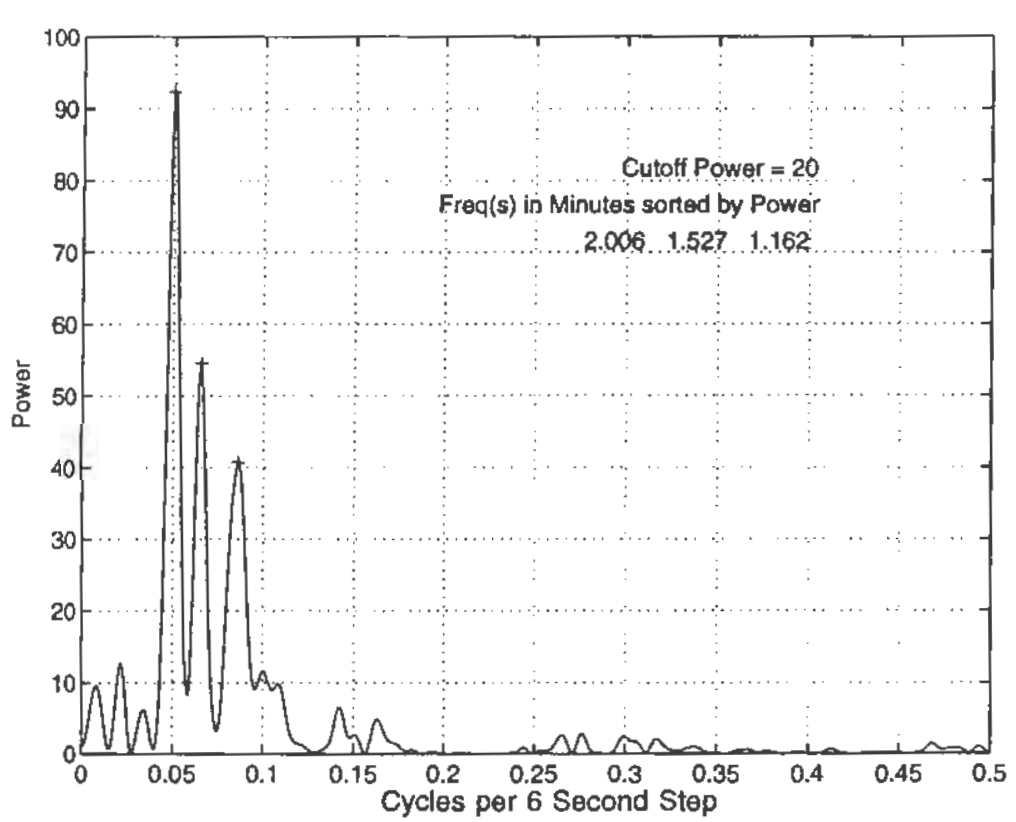

Average Reaction Time: 584.464

Reaction Time FFT

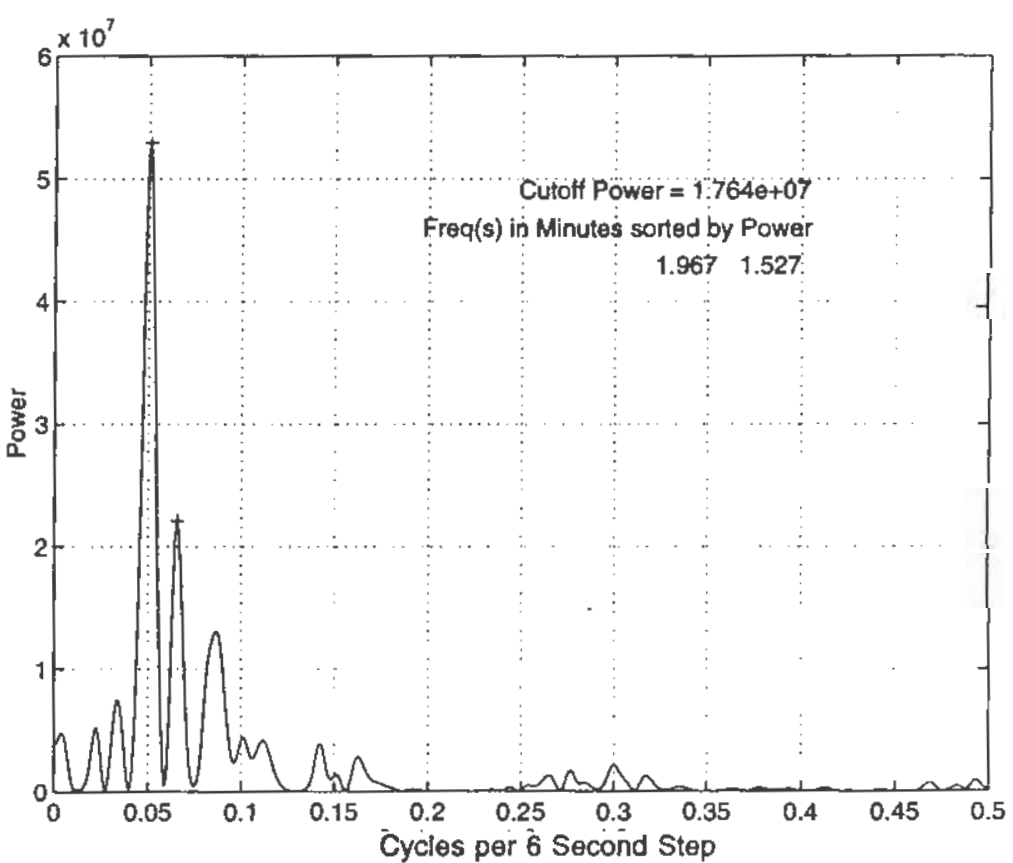




\section{Human Subject j017, Group 1}

Hit Rate: 0.632

Accuracy FFT

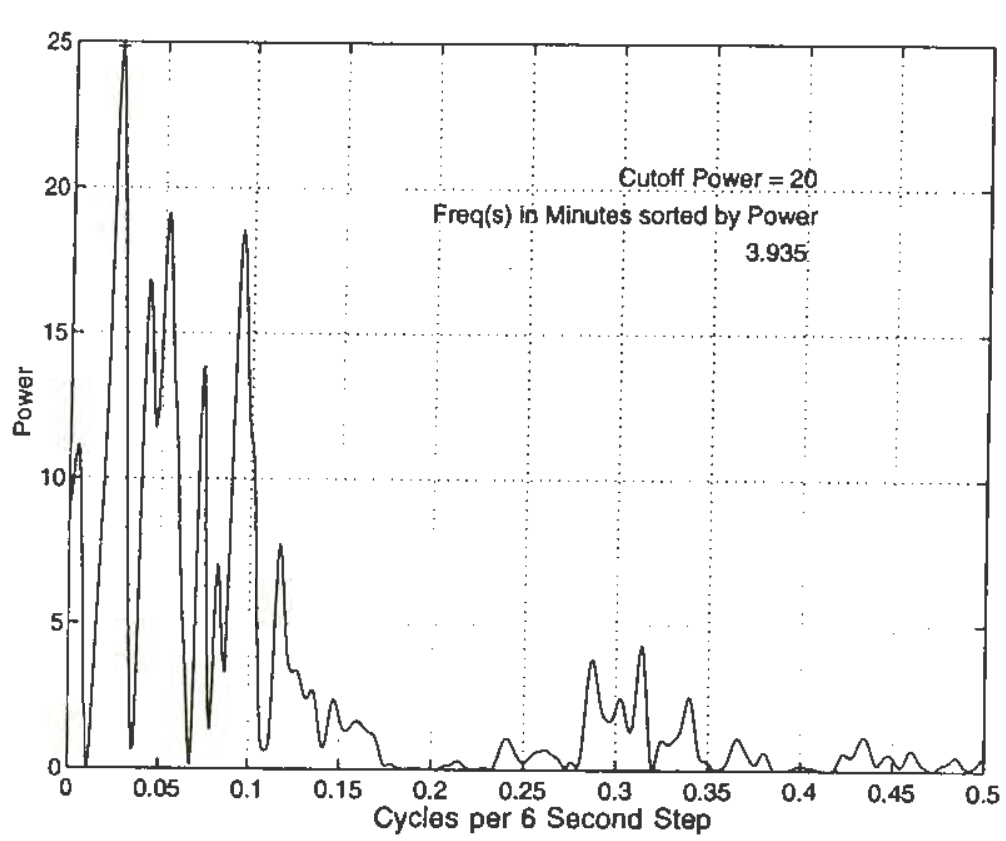

Average Reaction Time: 625.727

Reaction Time FFT

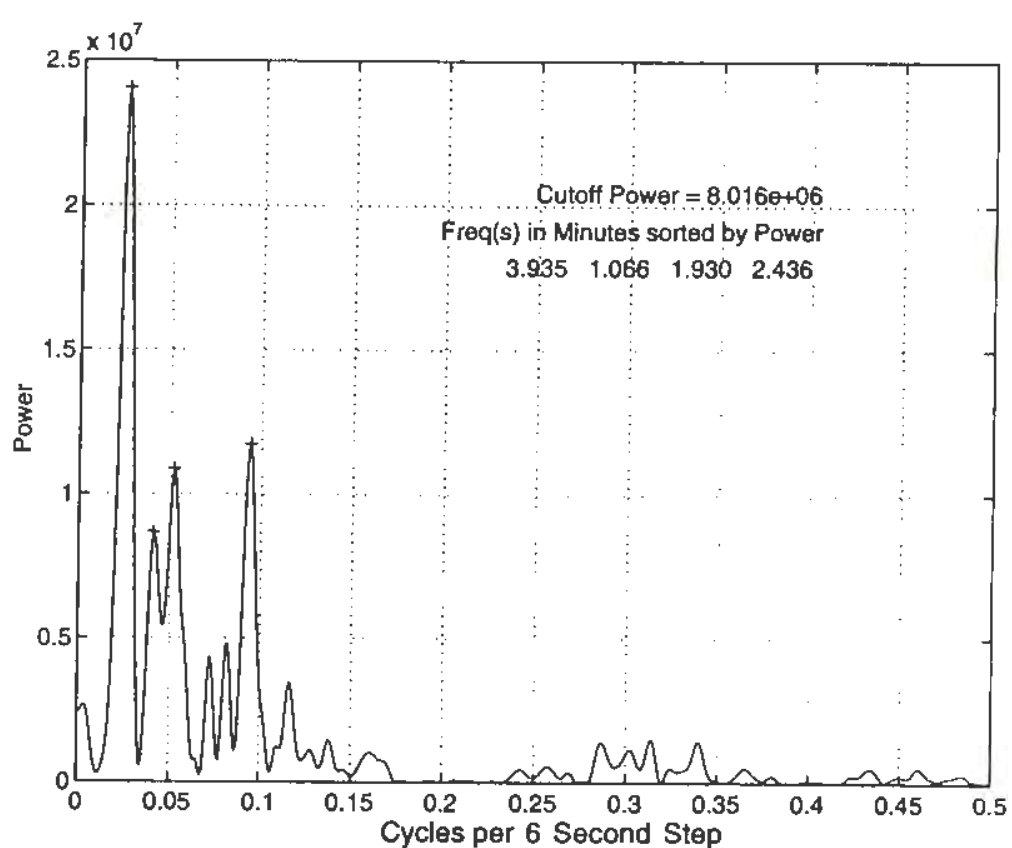




\section{Human Subject j018, Group 2}

Hit Rate: 0.784

Accuracy FFT

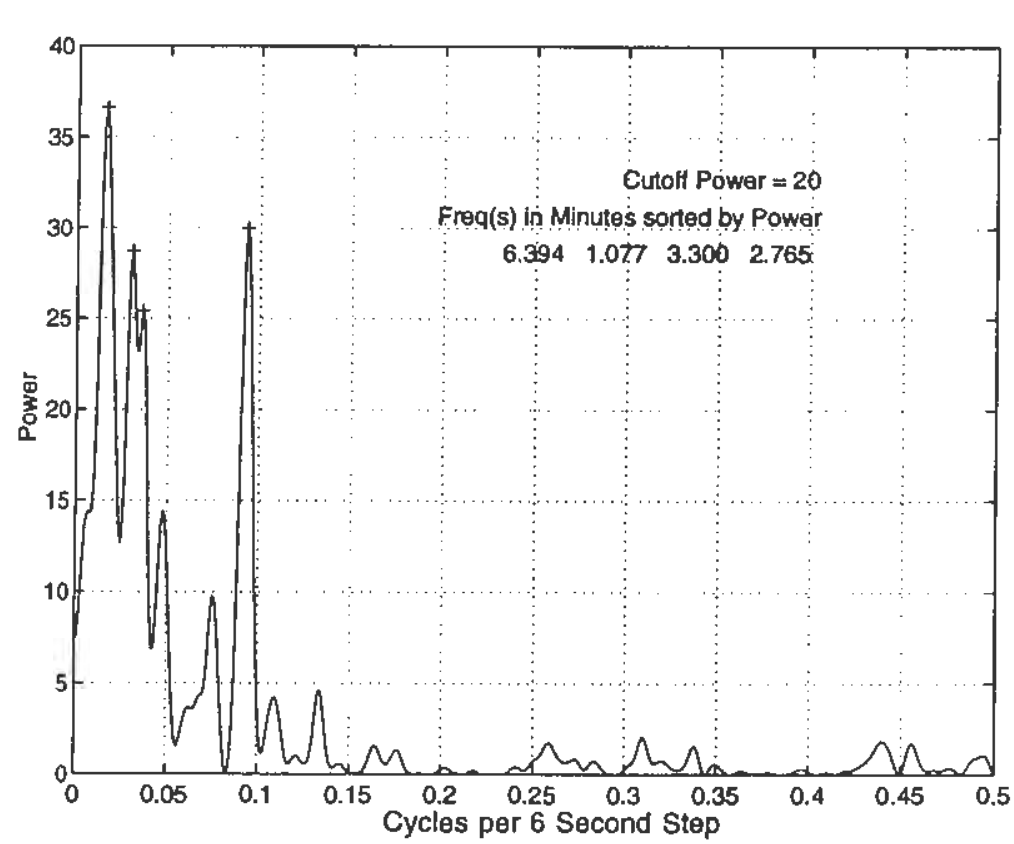

Average Reaction Time: 794.869

\section{Reaction Time FFT}

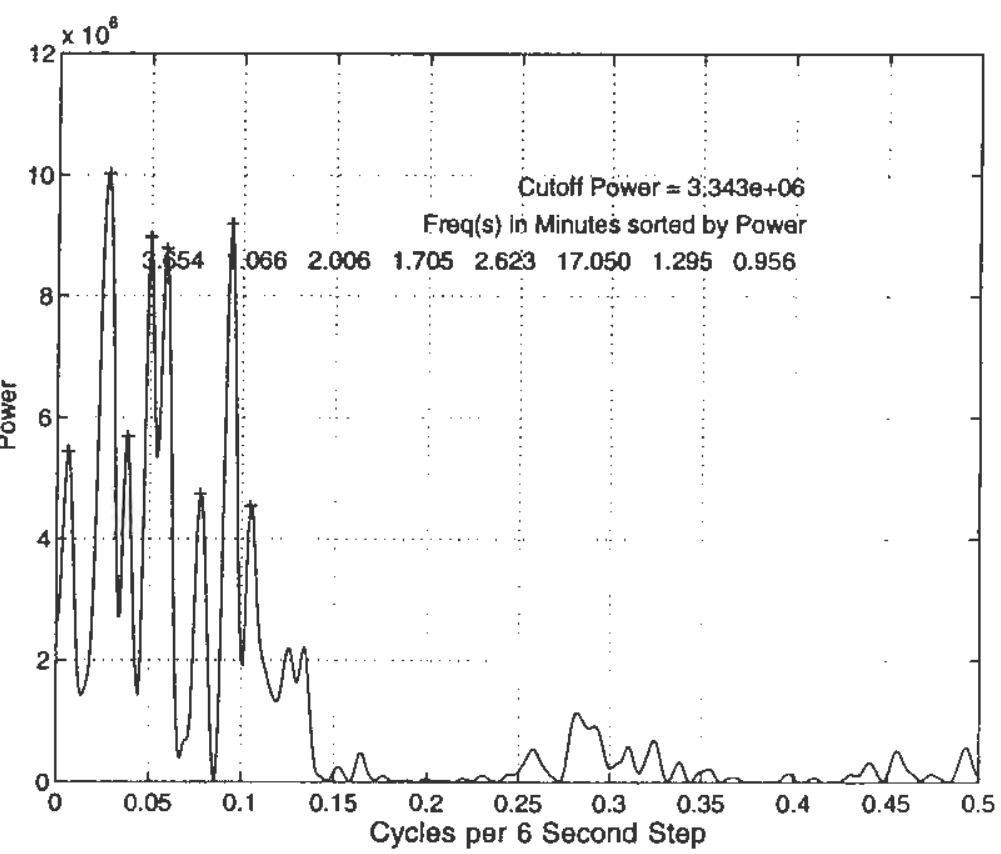




\section{Human Subjecct j020, Group 1}

Hit Rate: 0.665

Accuracy FFT

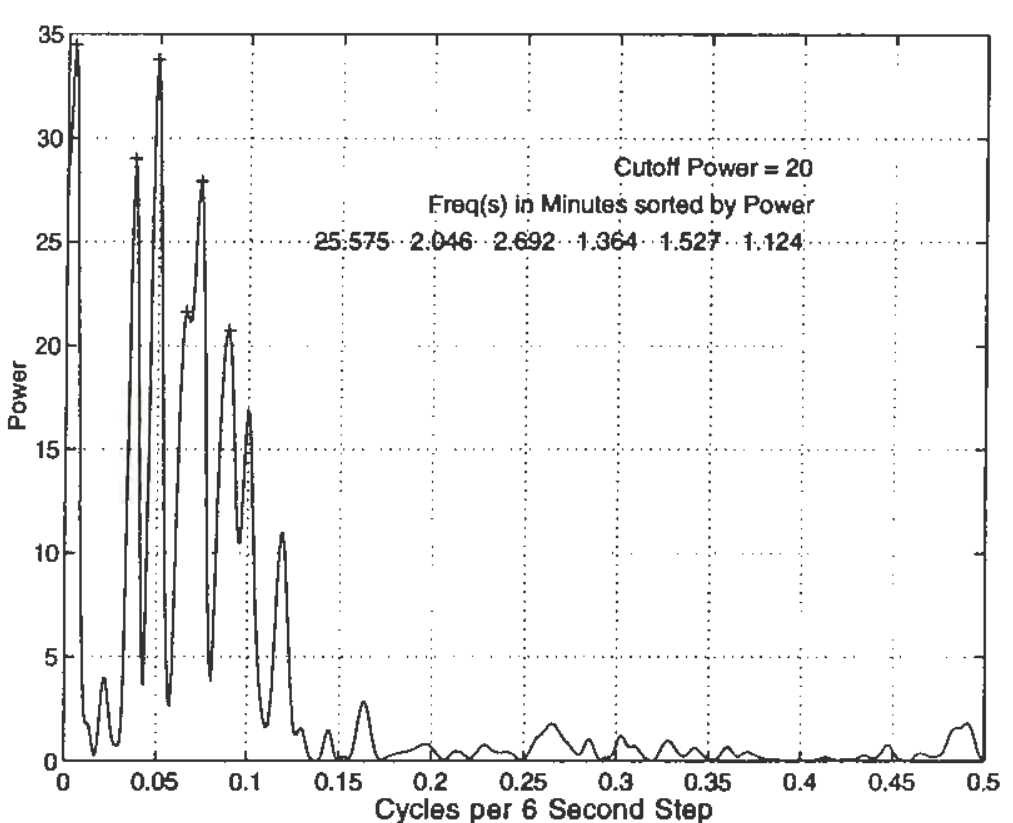

Average Reaction Time: 724.472

Reaction Time FFT

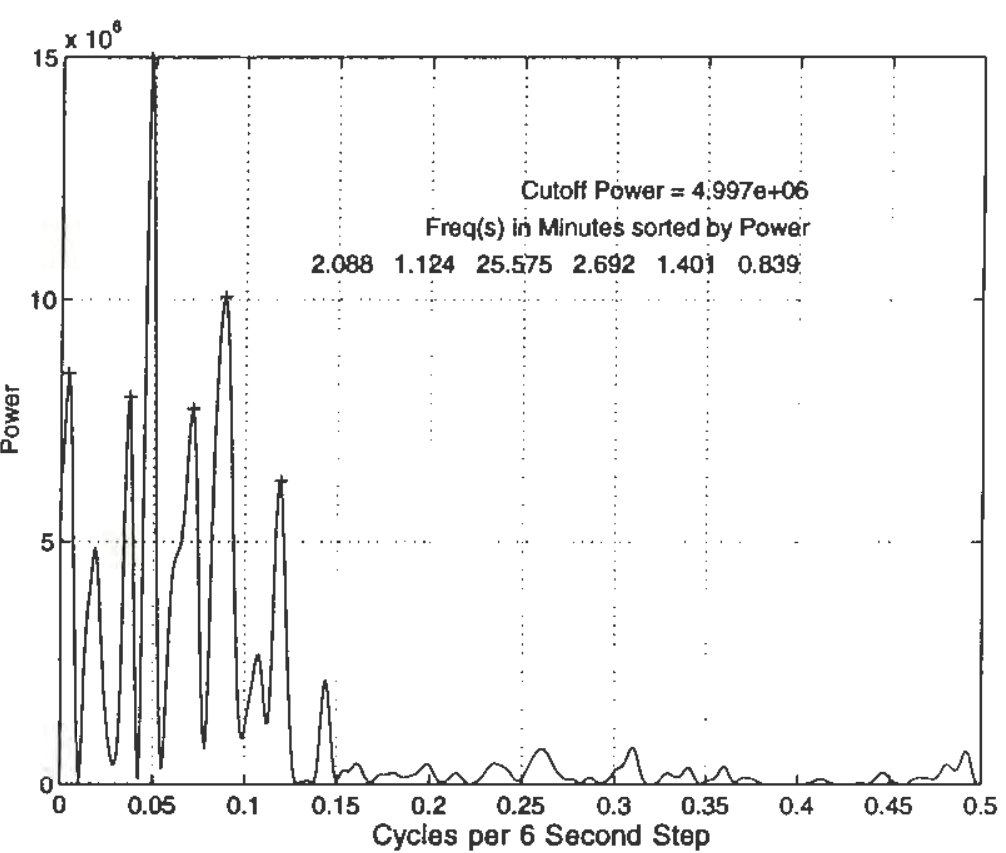




\section{Human Subject j021, Group 4}

Hit Rate: 0.881

Accuracy FFT

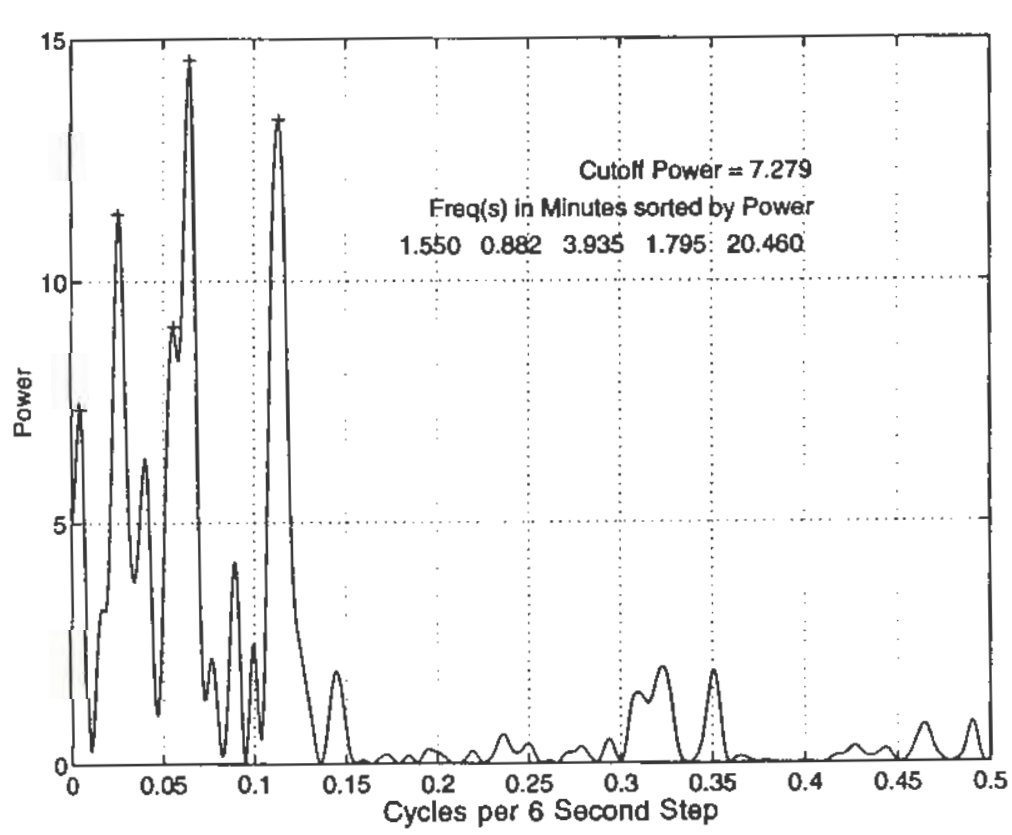

Average Reaction Time: 706.828

Reaction Time FFT

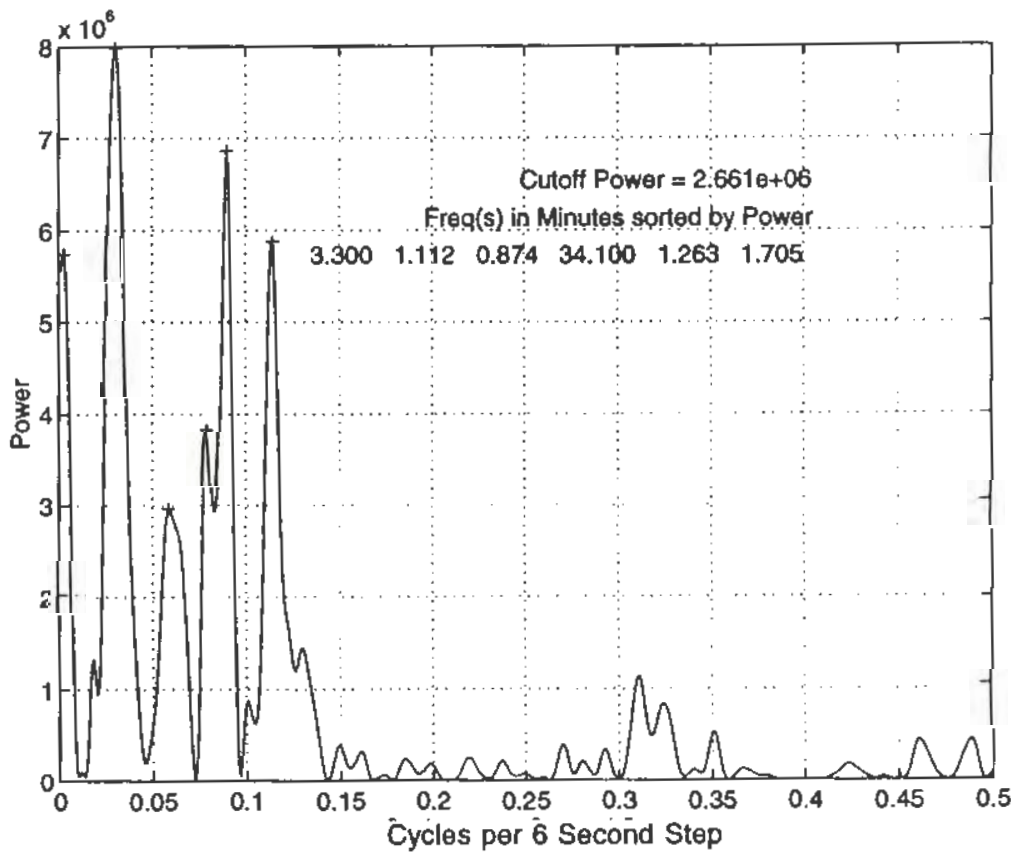




\section{Human Subject j022, Group 2}

Hit Rate: 0.795

Accuracy FFT

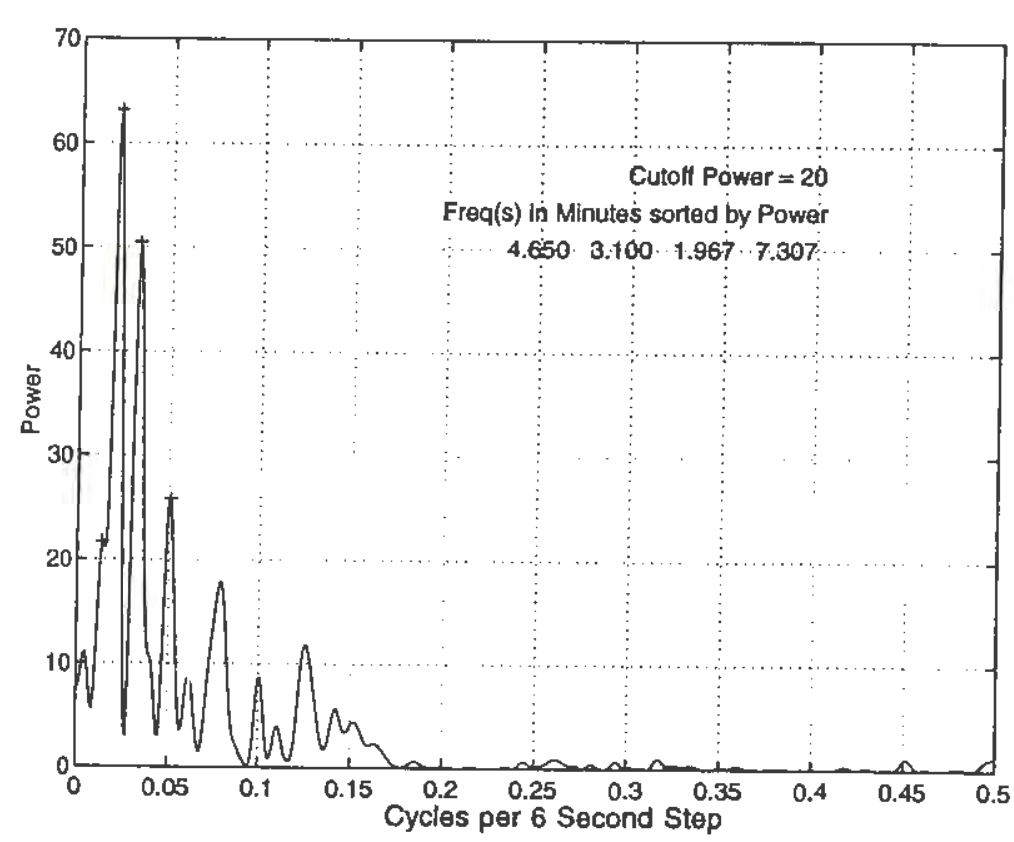

Average Reaction Time: 720.973

Reaction Time FFT

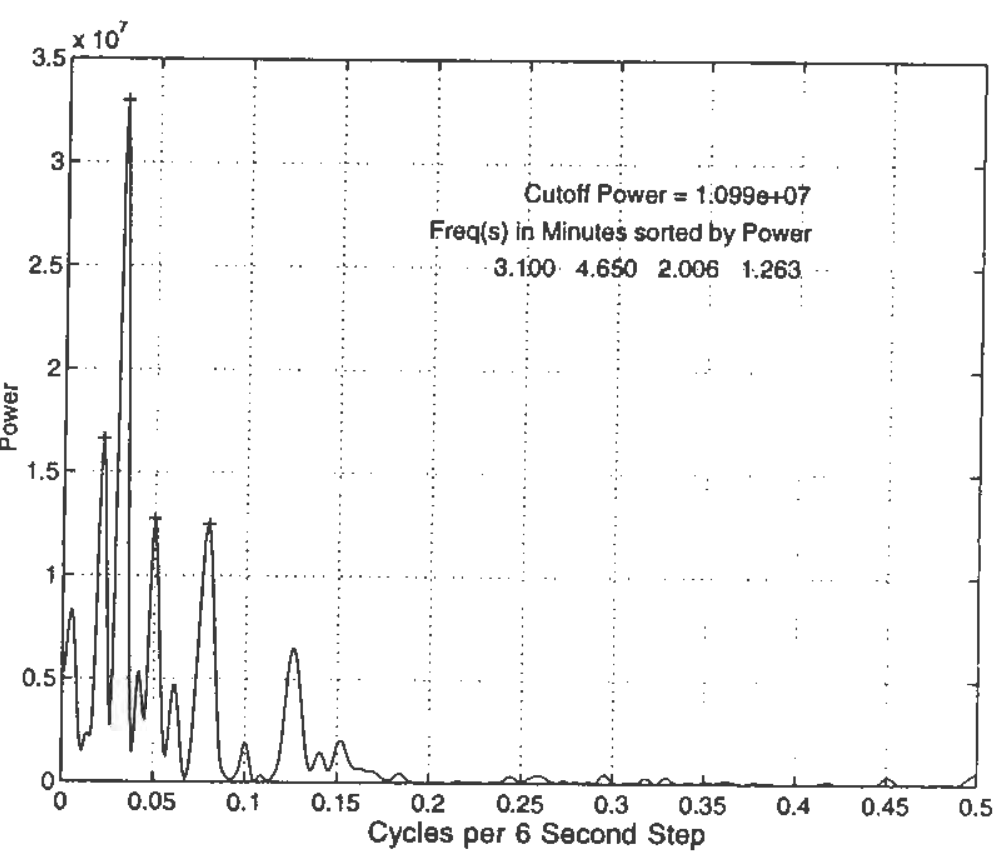




\section{Human Subject j023, Group 4}

Hit Rate: 0.881

Accuracy FFT

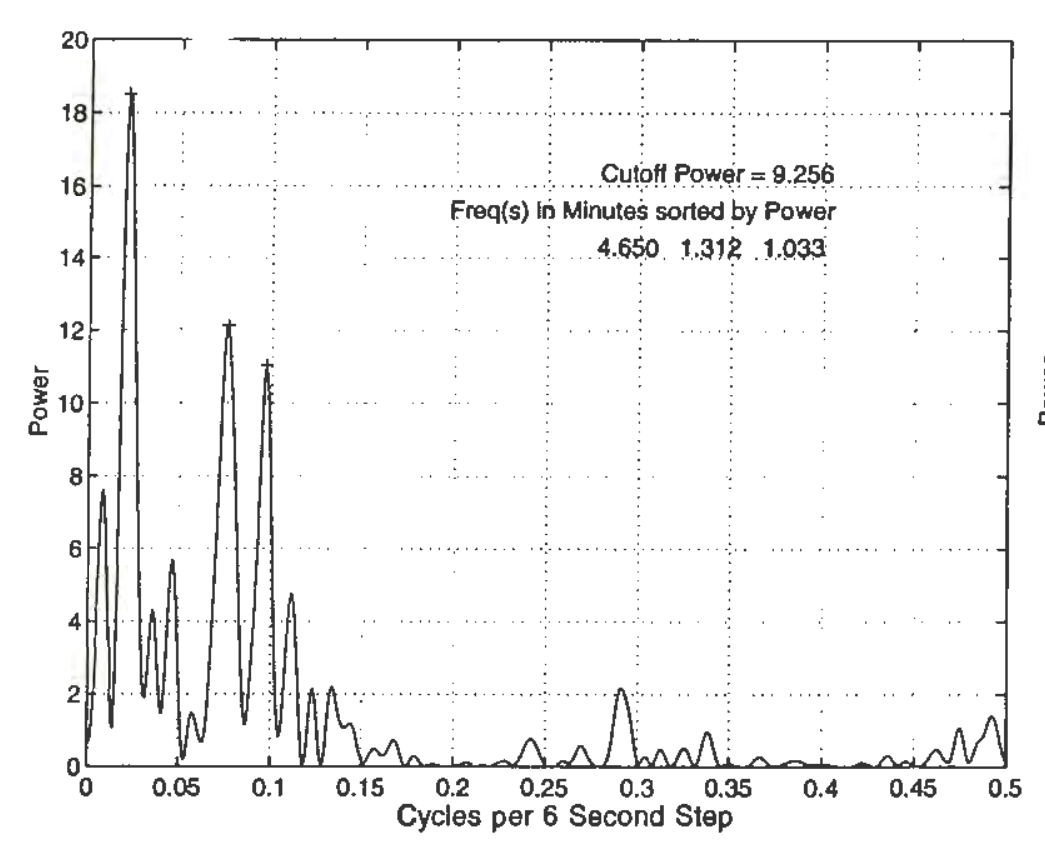

Average Reaction Time: 706.307

Reaction Time FFT

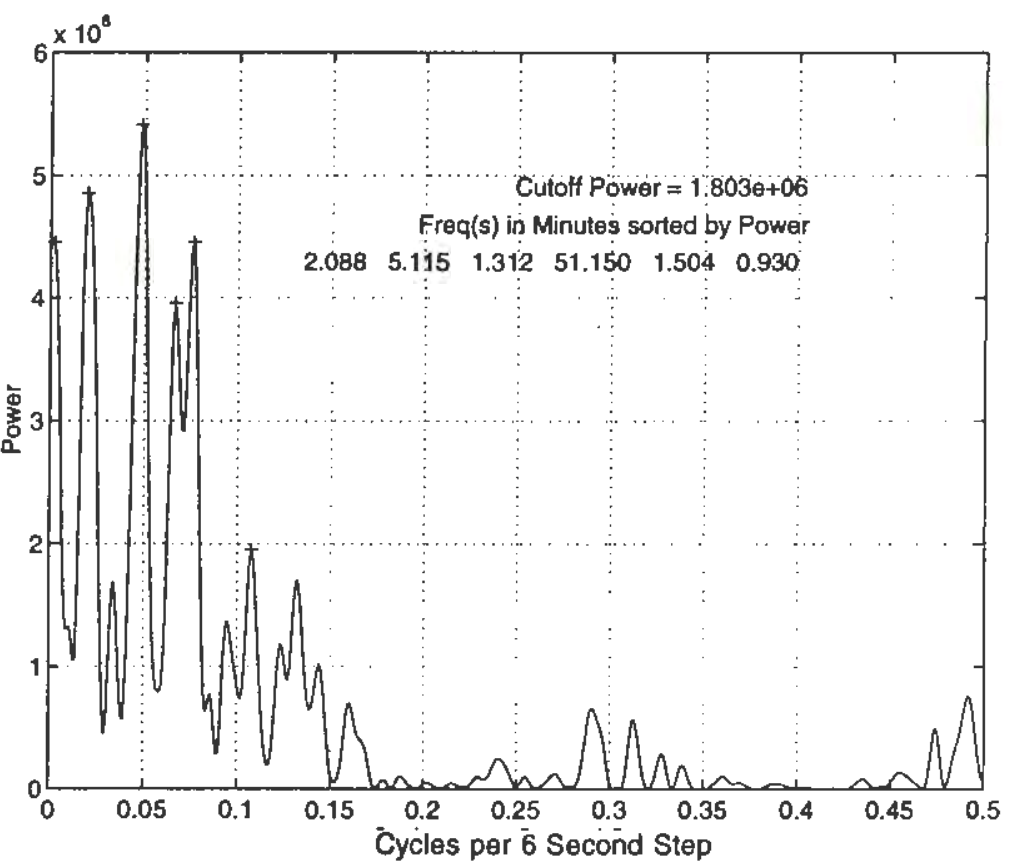




\section{Human Subject j024, Group 2}

Hit Rate: 0.762

Accuracy FFT

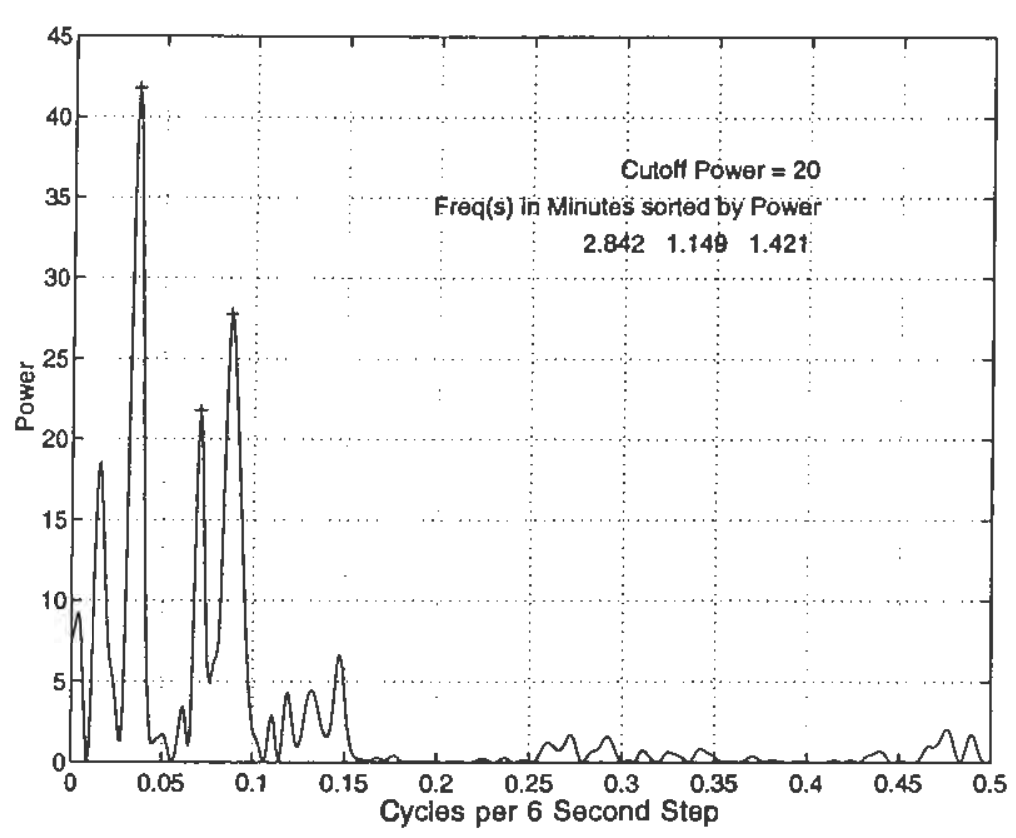

Average Reaction Time: 719.113

Reaction Time FFT

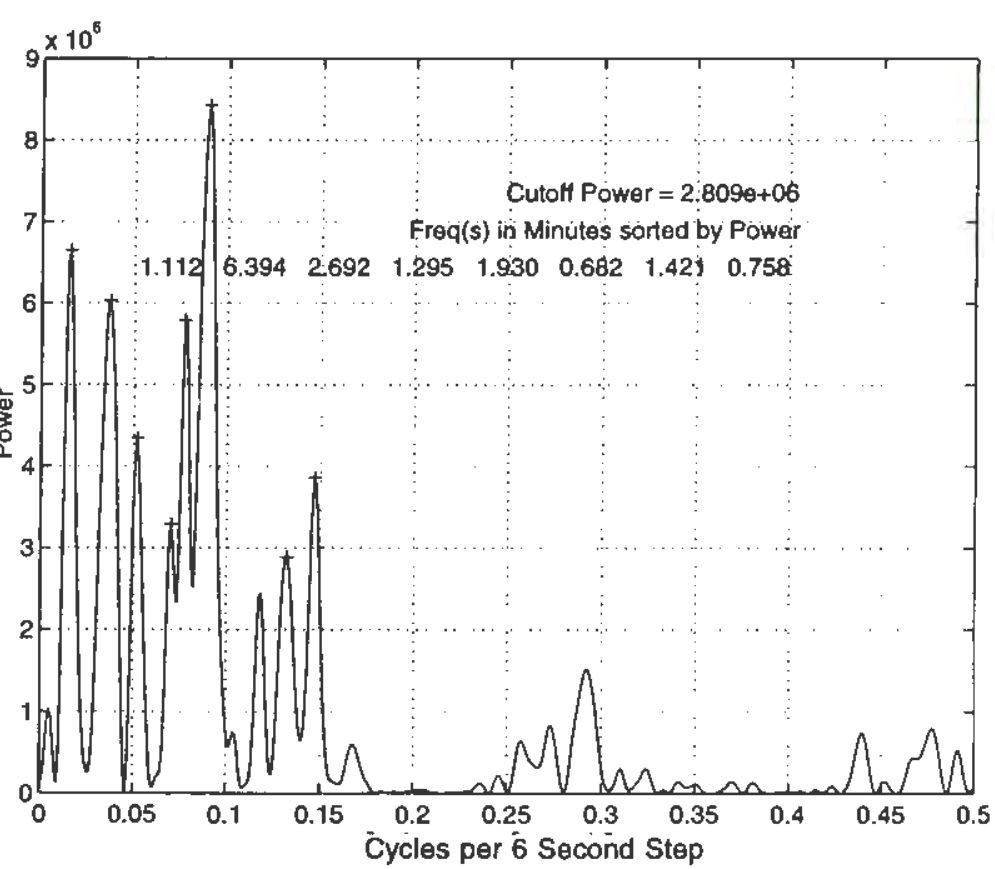




\section{Human Subject j025, Group 1}

Hit Rate: 0.611

\section{Accuracy FFT}

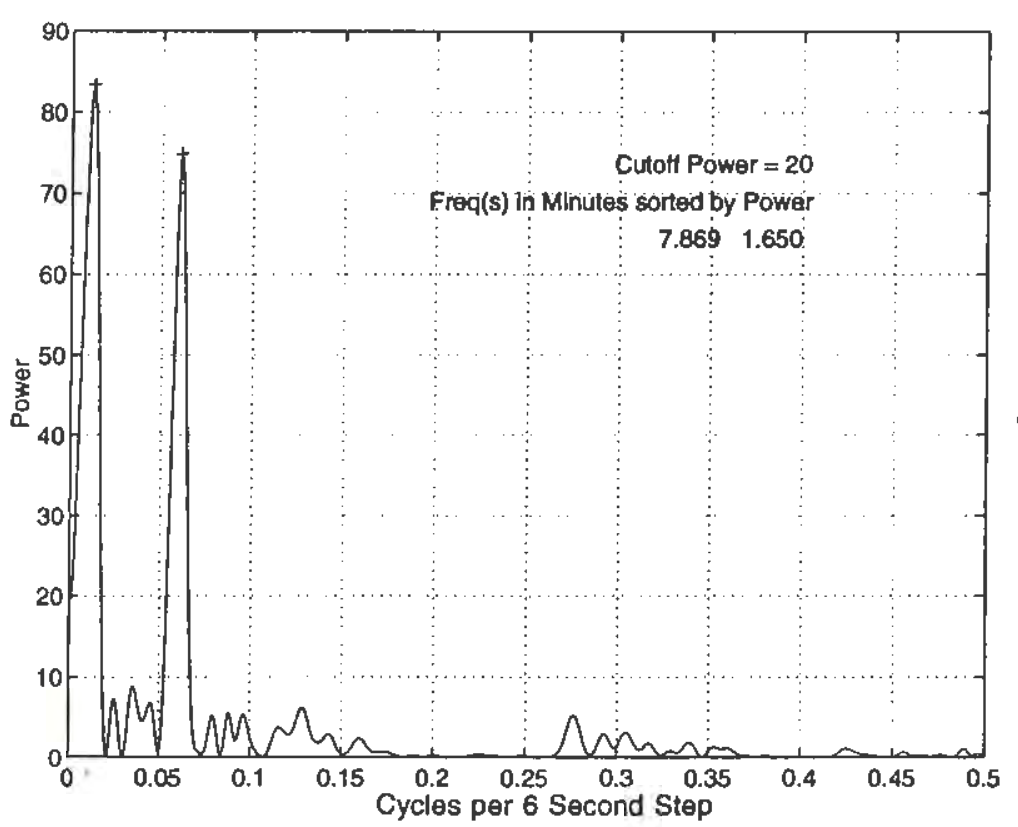

Average Reaction Time: 909.204

Reaction Time FFT

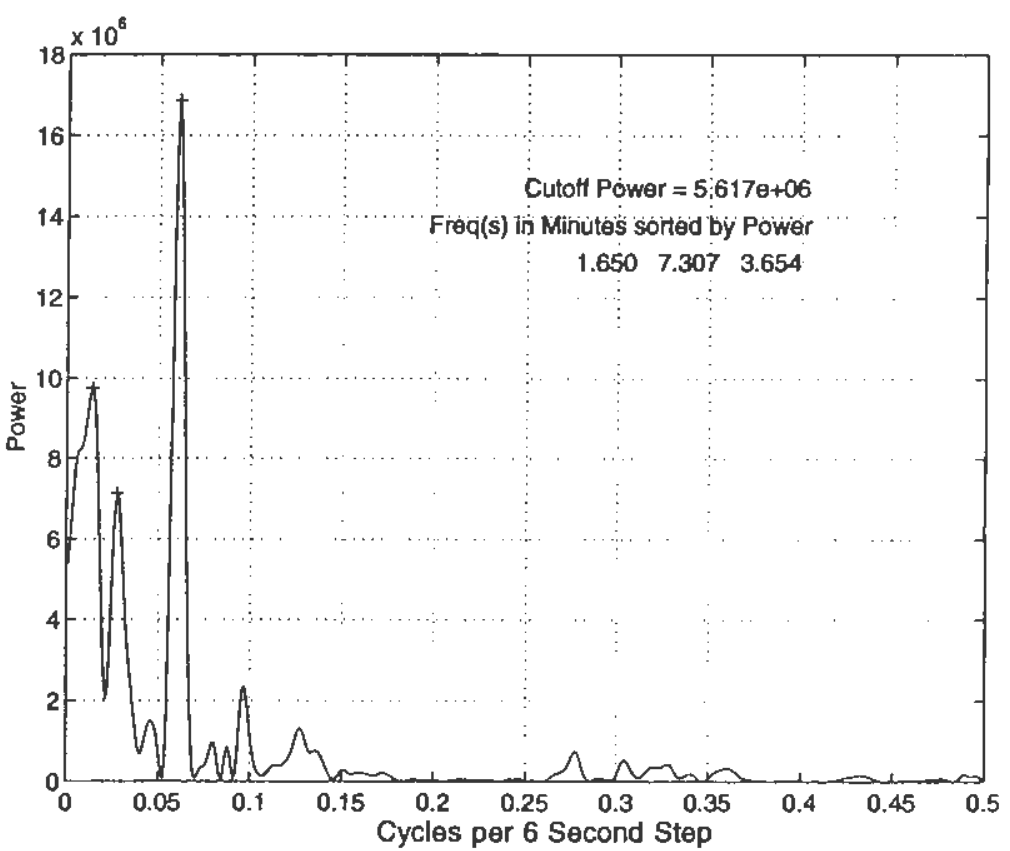




\section{Human Subject j026, Group 3}

Hit Rate: 0.838

Accuracy FFT

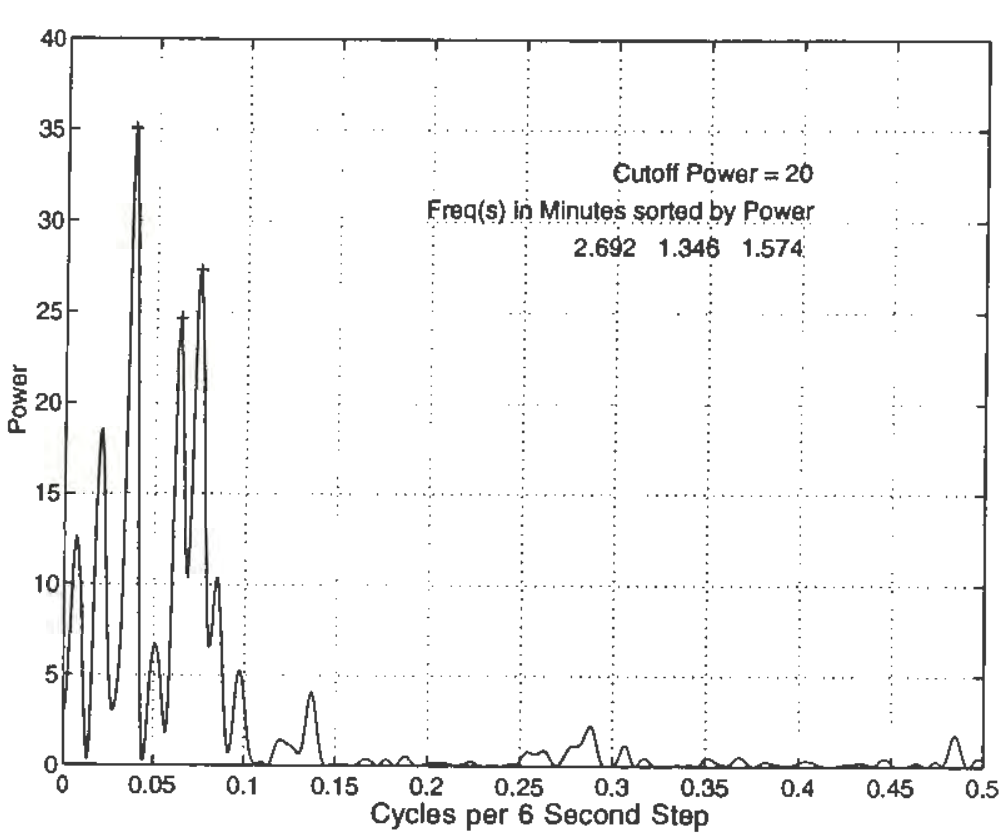

Average Reaction Time: 758.239

Reaction Time FFT

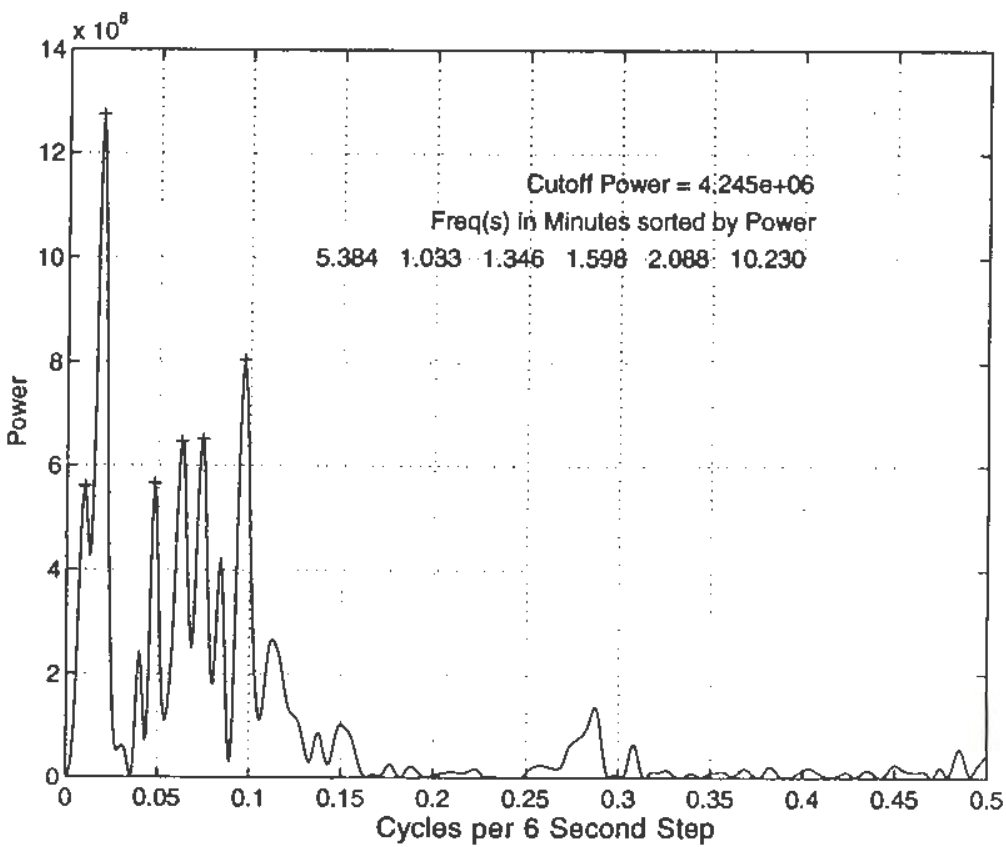




\section{Human Subject j028, Group 2}

Hit Rate: 0.789

Accuracy FFI

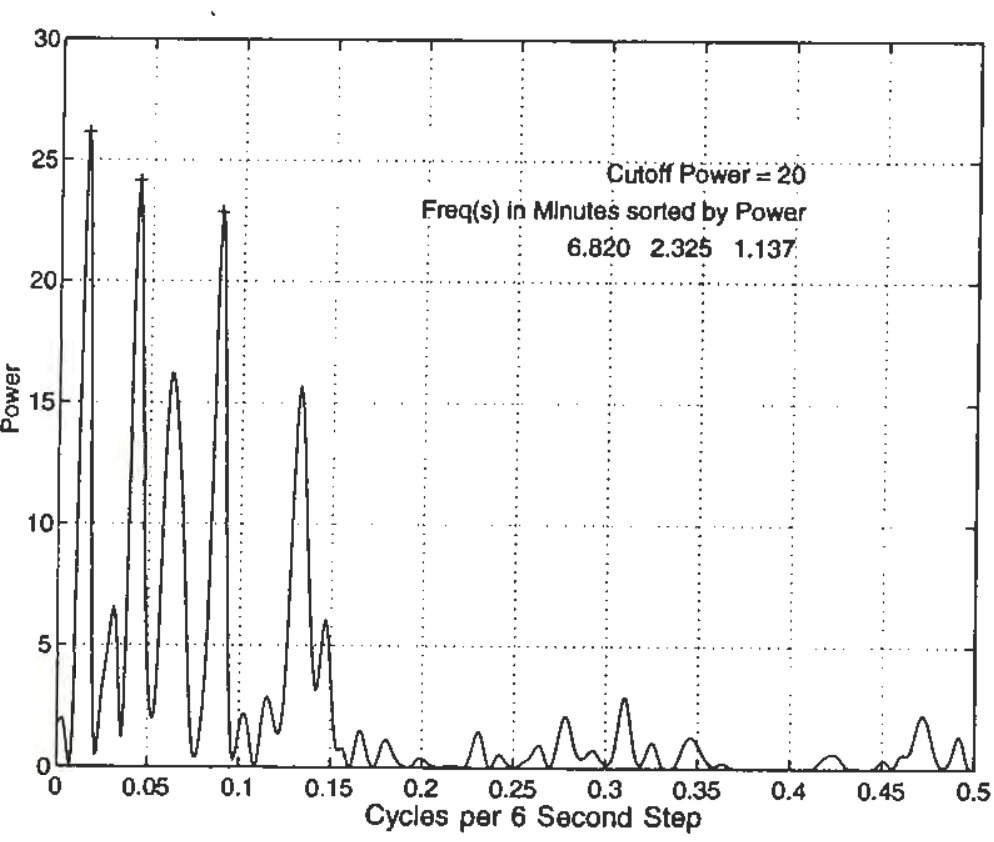

Average Reaction Time: 693.425

Reaction Time FFT

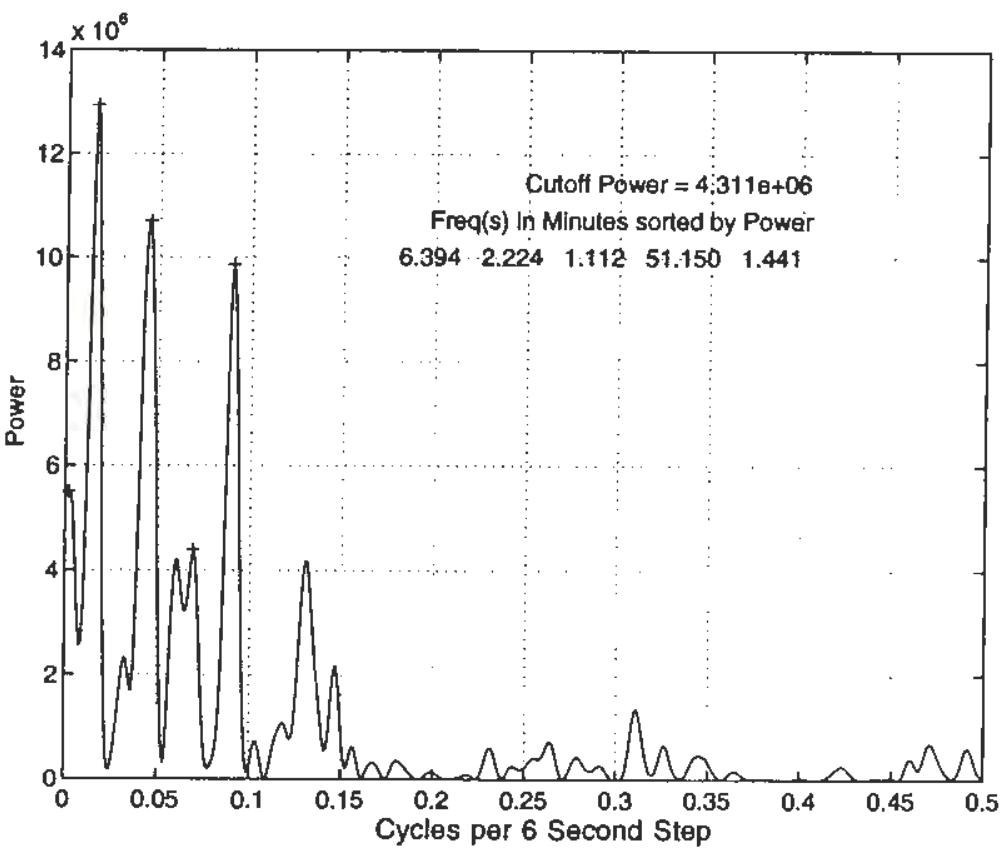




\section{Human Subject j030, Group 2}

Hit Rate: 0.778

\section{Accuracy FFT}

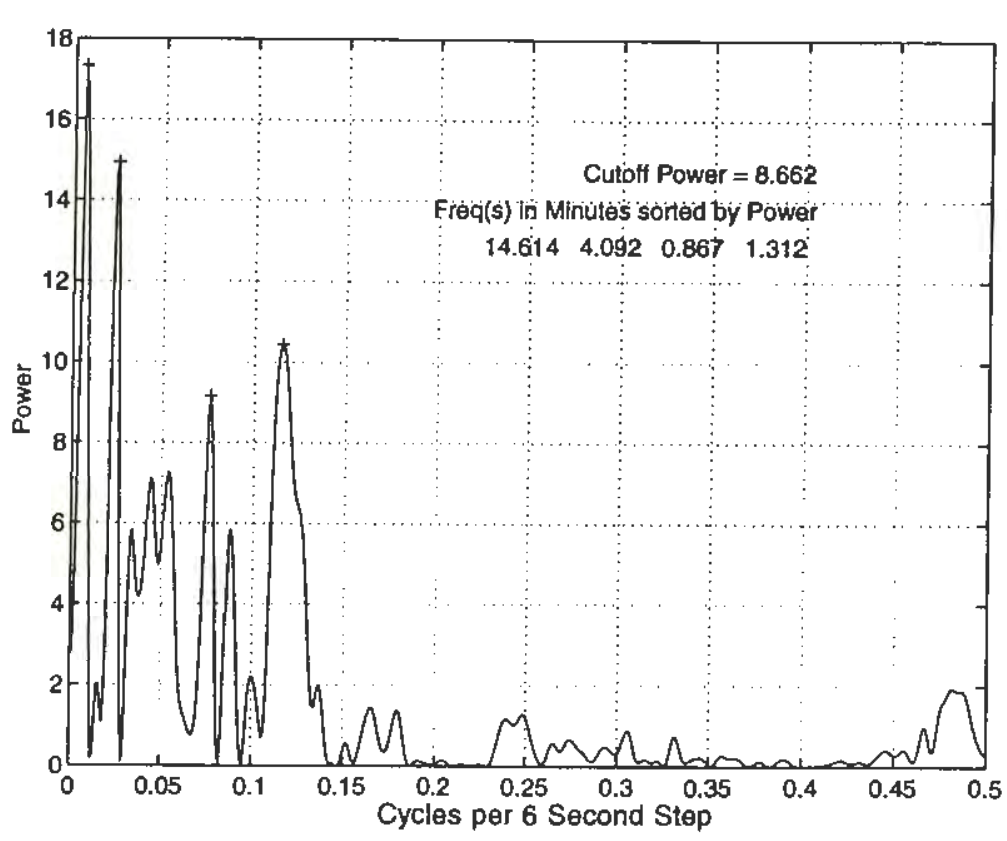

Average Reaction Time: 683.938

\section{Reaction Time FFT}

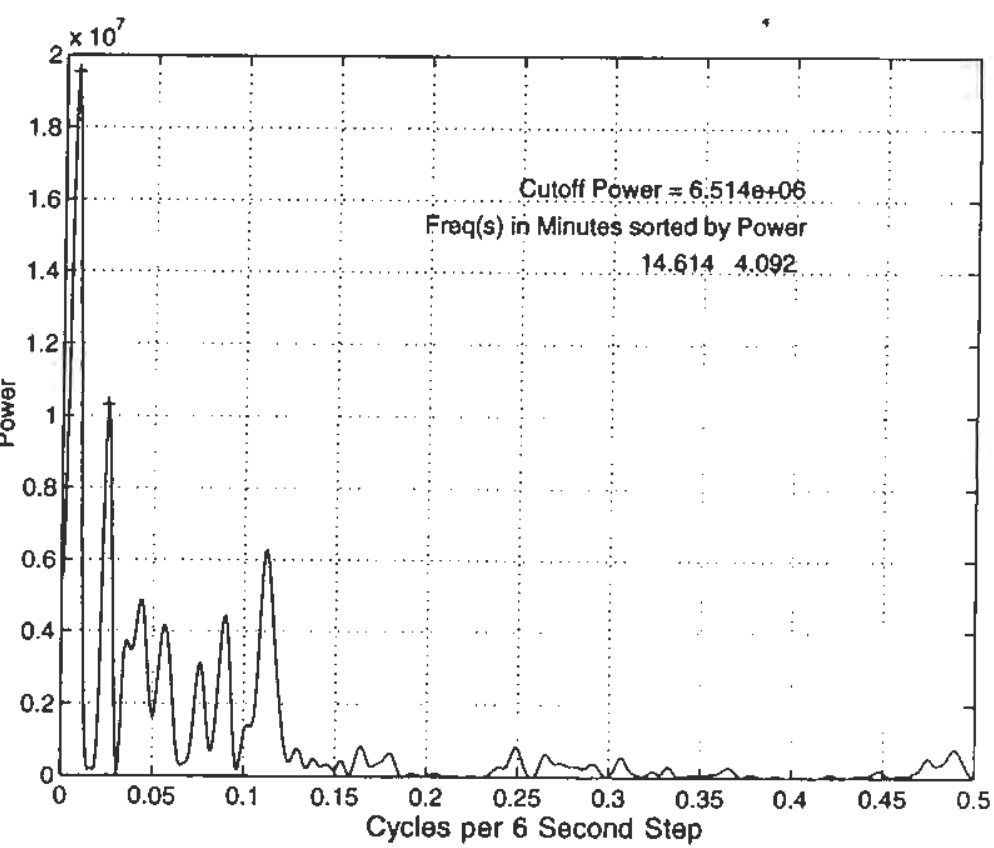




\section{Human Subject j031, Group 3}

Hit Rate: 0.87

\section{Accuracy FFT}

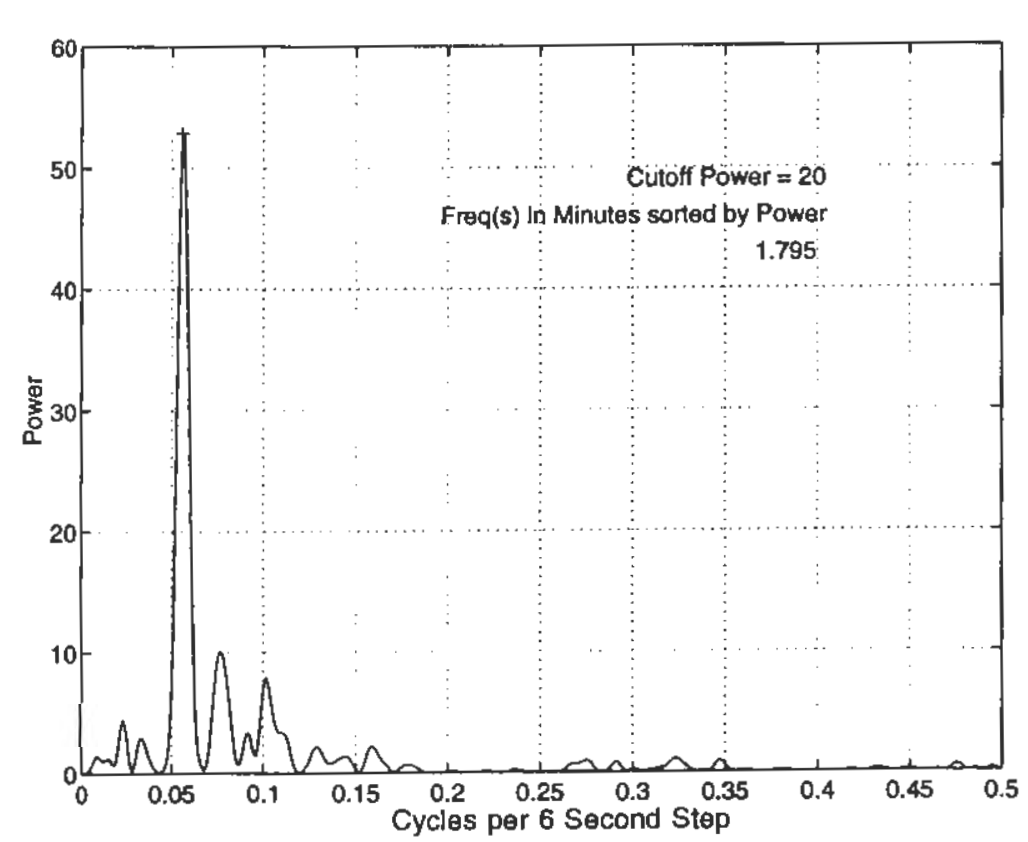

Average Reaction Time: 844.143

\section{Reaction Time FFT}

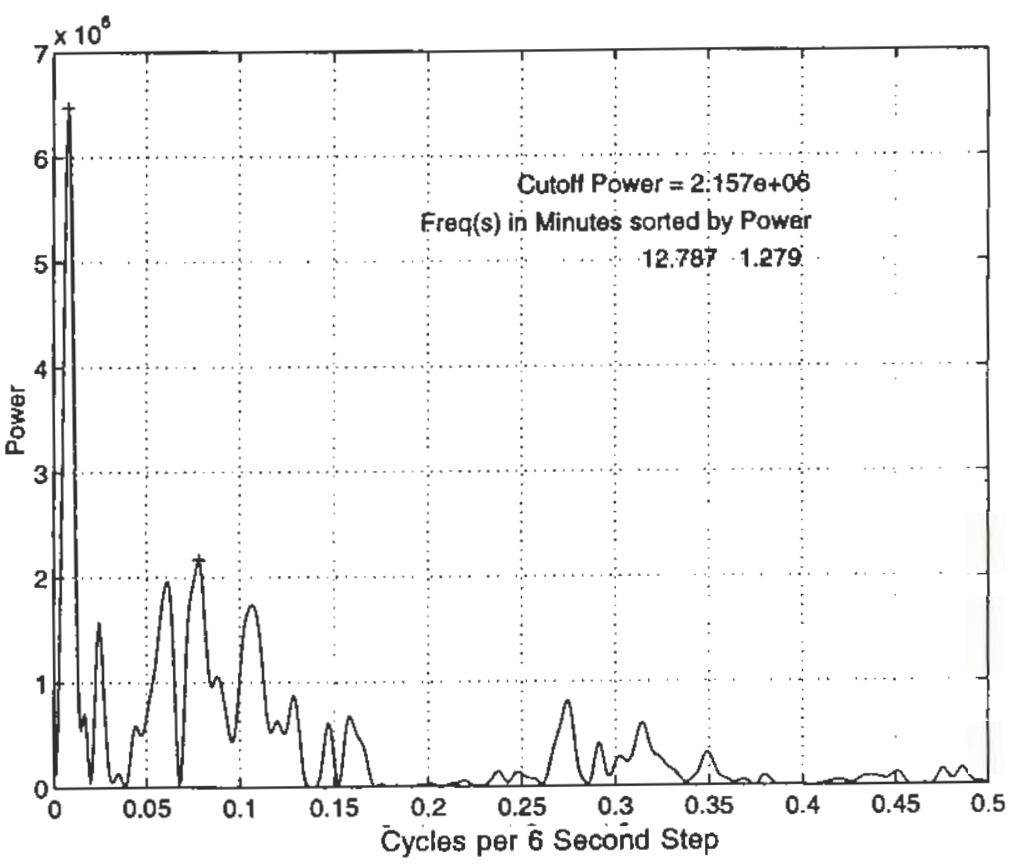




\section{Human Subject j032, Group 2}

Hit Rate: 0.741

Accuracy FFT

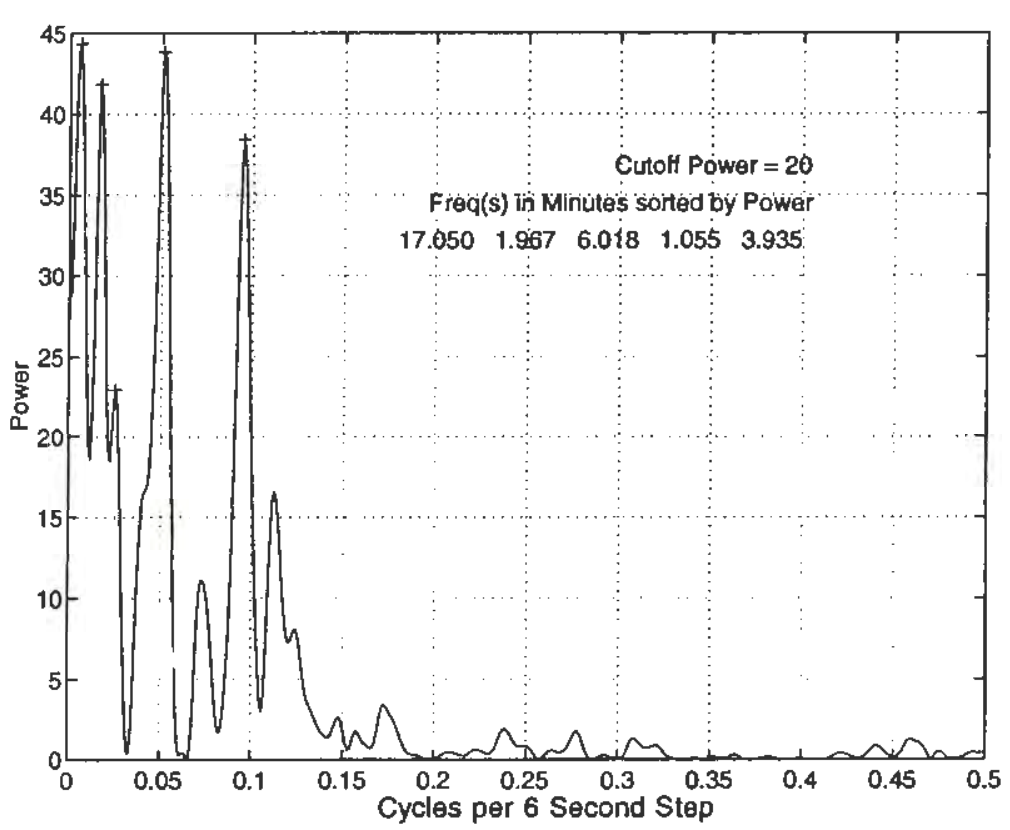

Average Reaction Time: 643.328

Reaction Time FFT

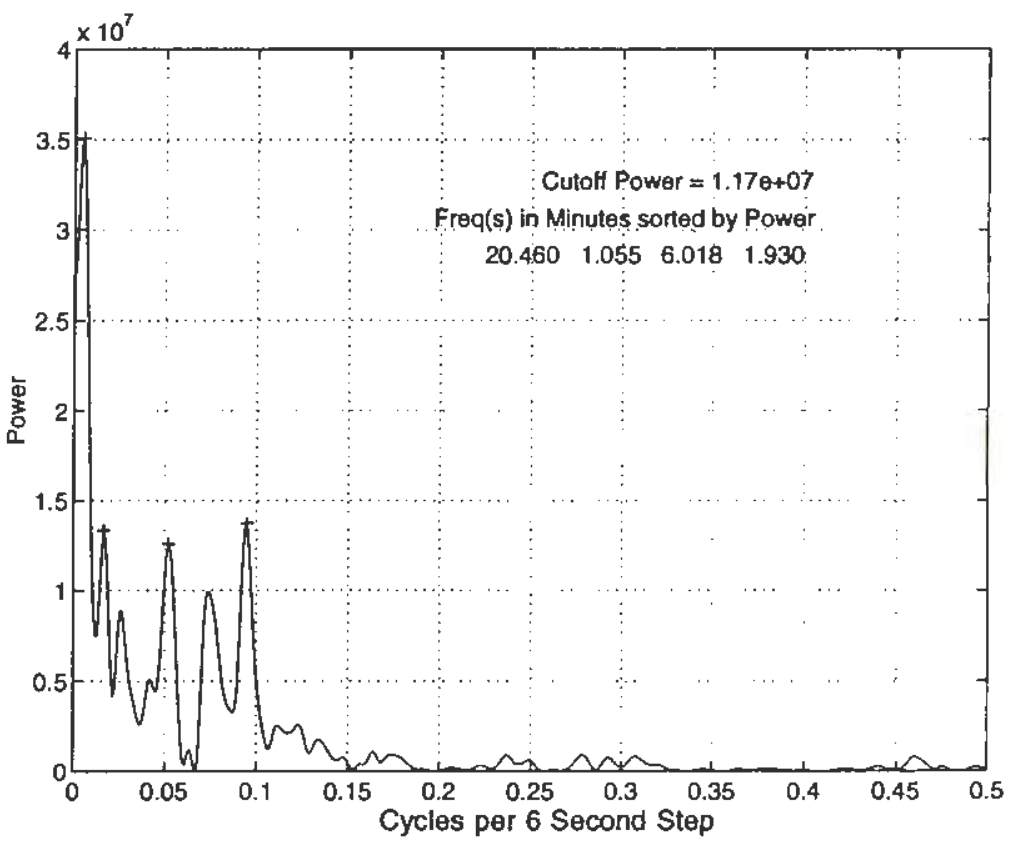




\section{Human Subject j034. Group 1}

Hit Rate: 0.676

Accuracy FFT

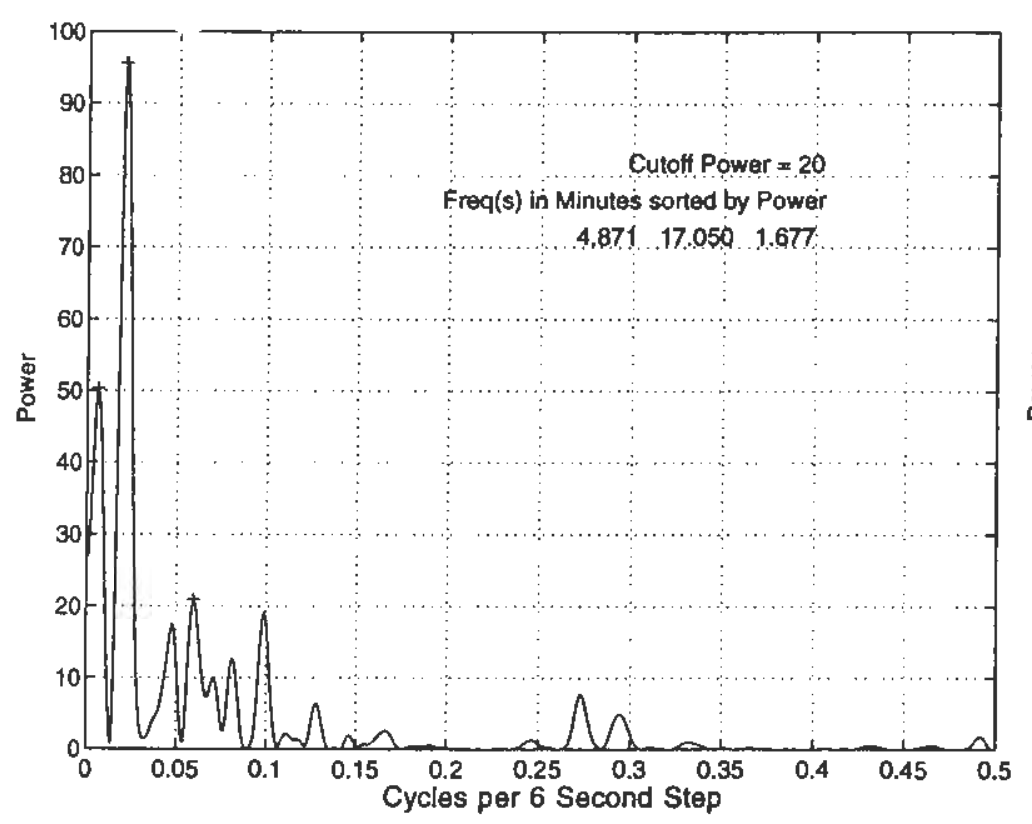

Average Reaction Time: 684.008

Reaction Time FFT

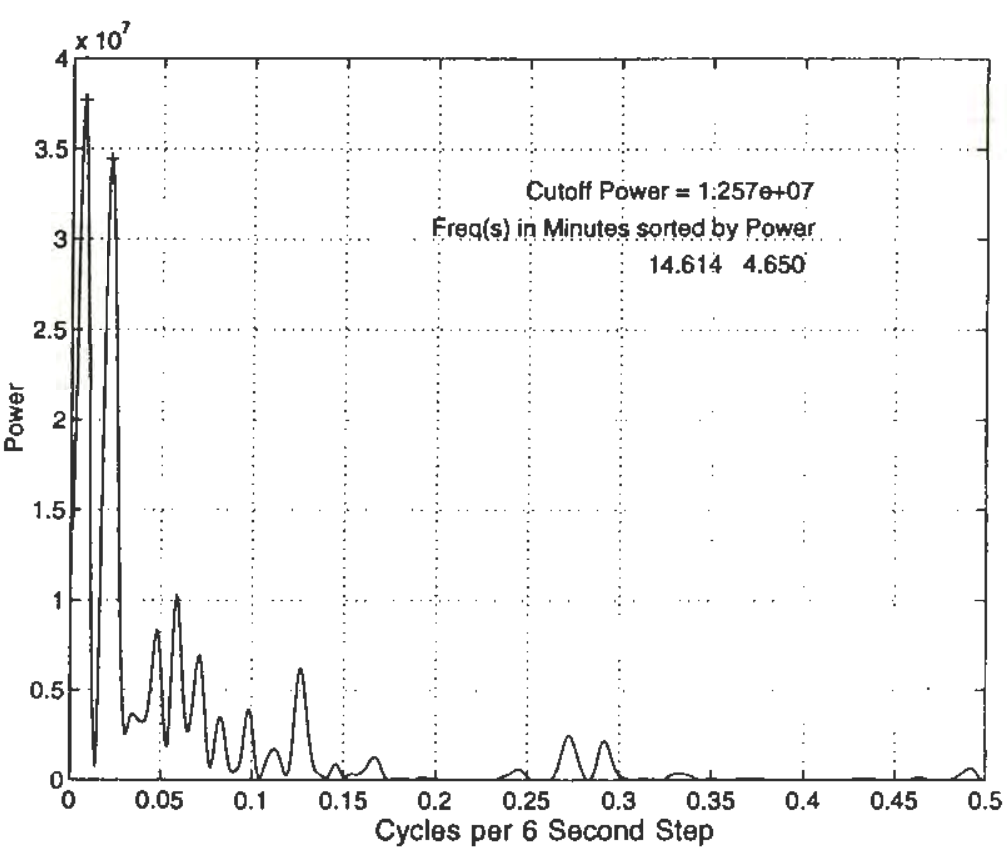




\section{Human Subject j035. Group 1}

Hit Rate: 0.67

Accuracy FFT

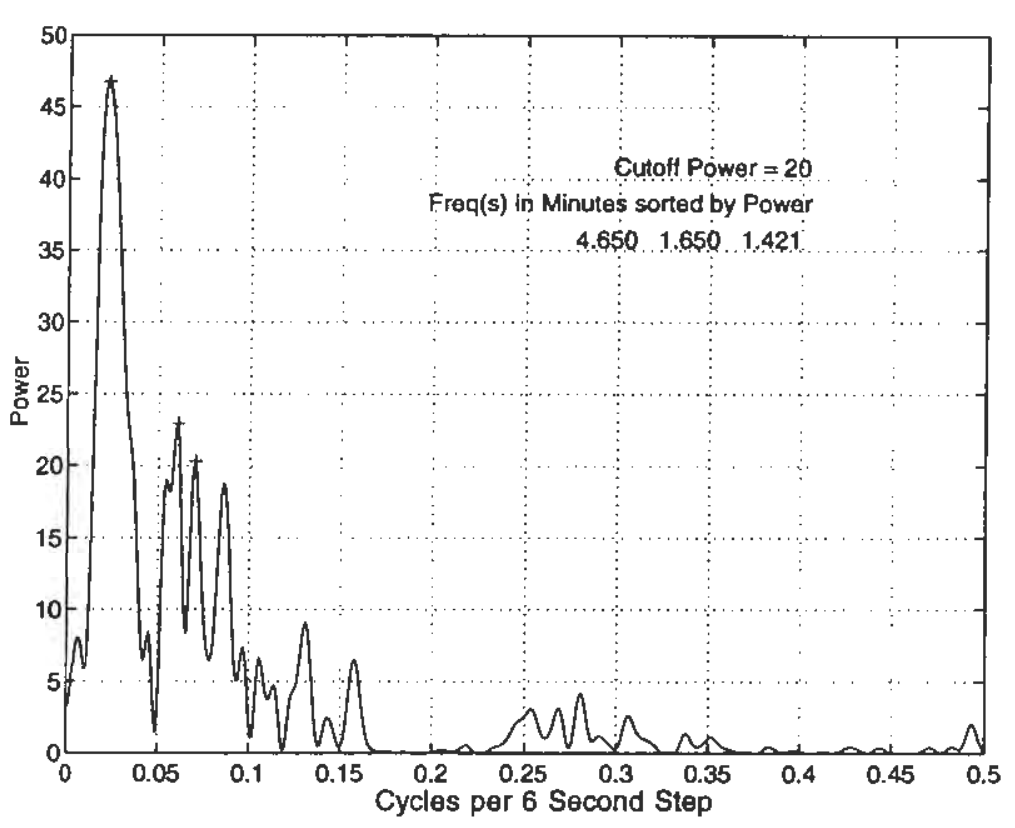

Average Reaction Time: 690.056

Reaction Time FFT

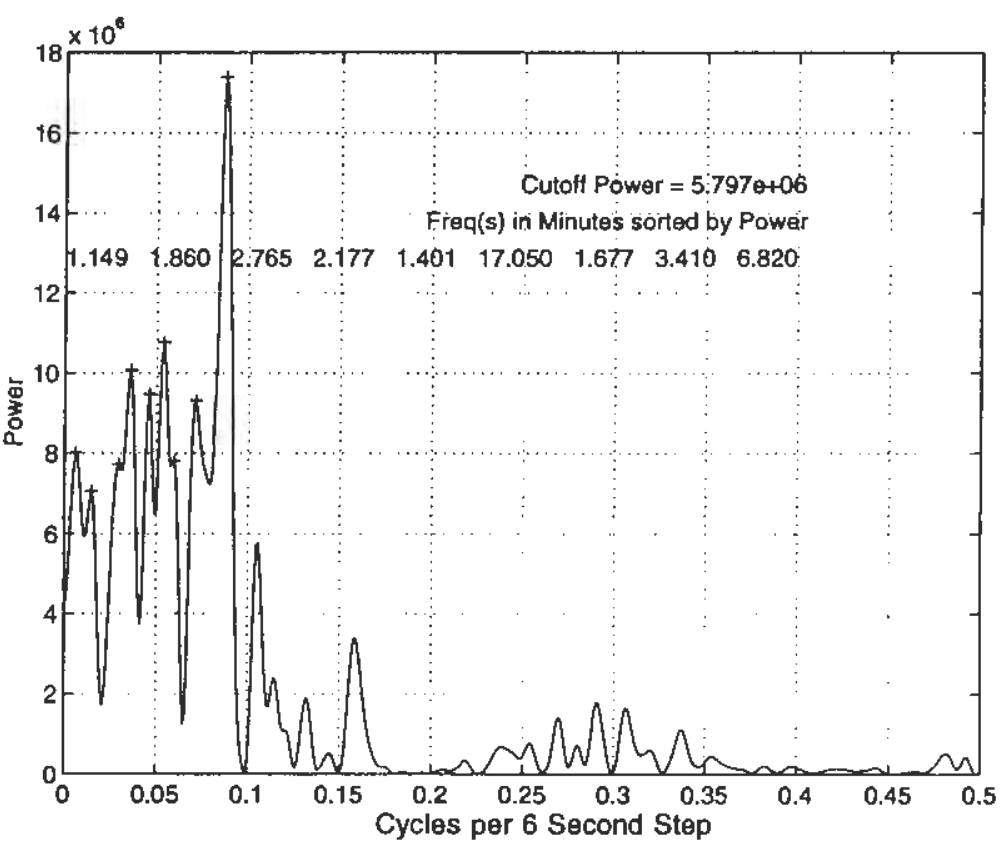




\section{Human Subject j036, Group 4}

Hit Rate: 0.892

Accuracy FFT

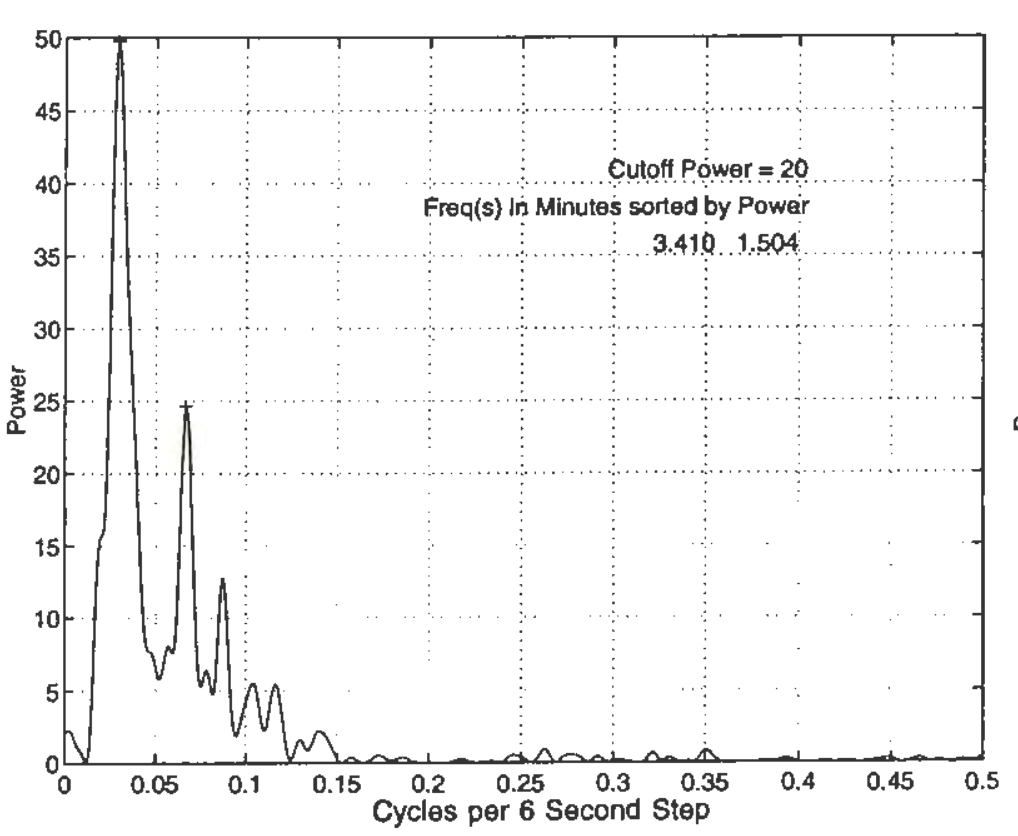

Average Reaction Time: 731.752

Reaction Time FFT

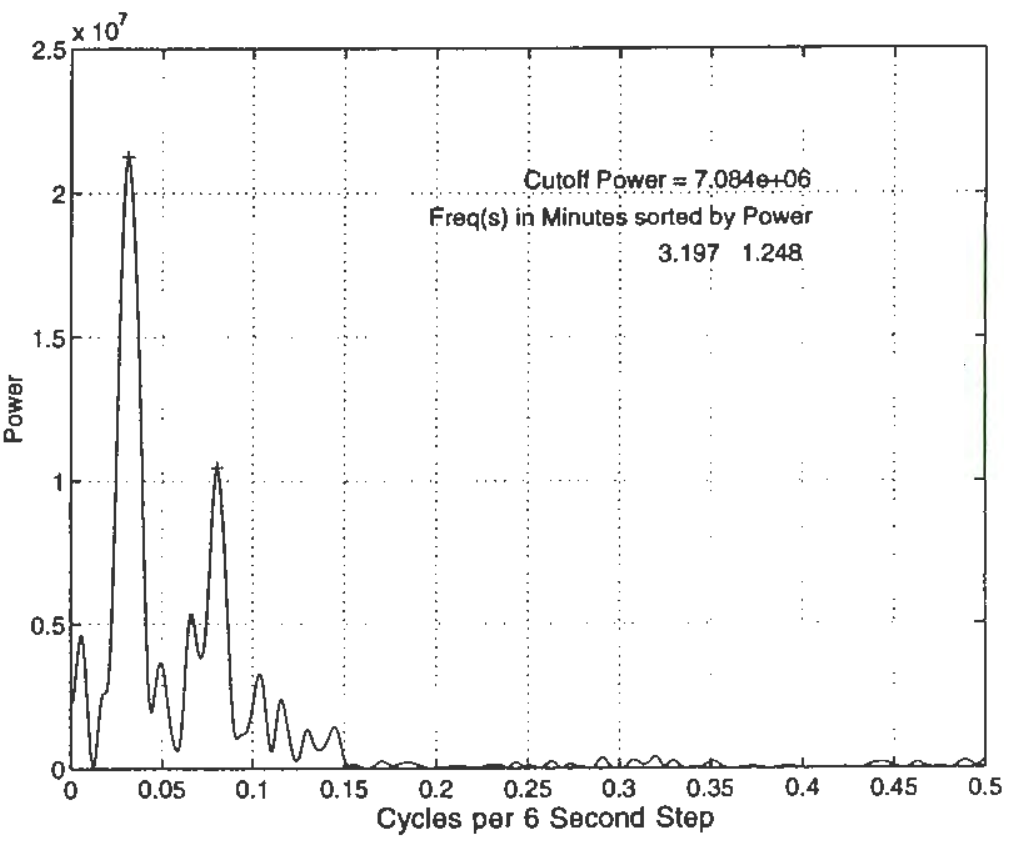




\section{Human Subject j037, Group 3}

Hit Rate: 0.854

\section{Accuracy FFT}

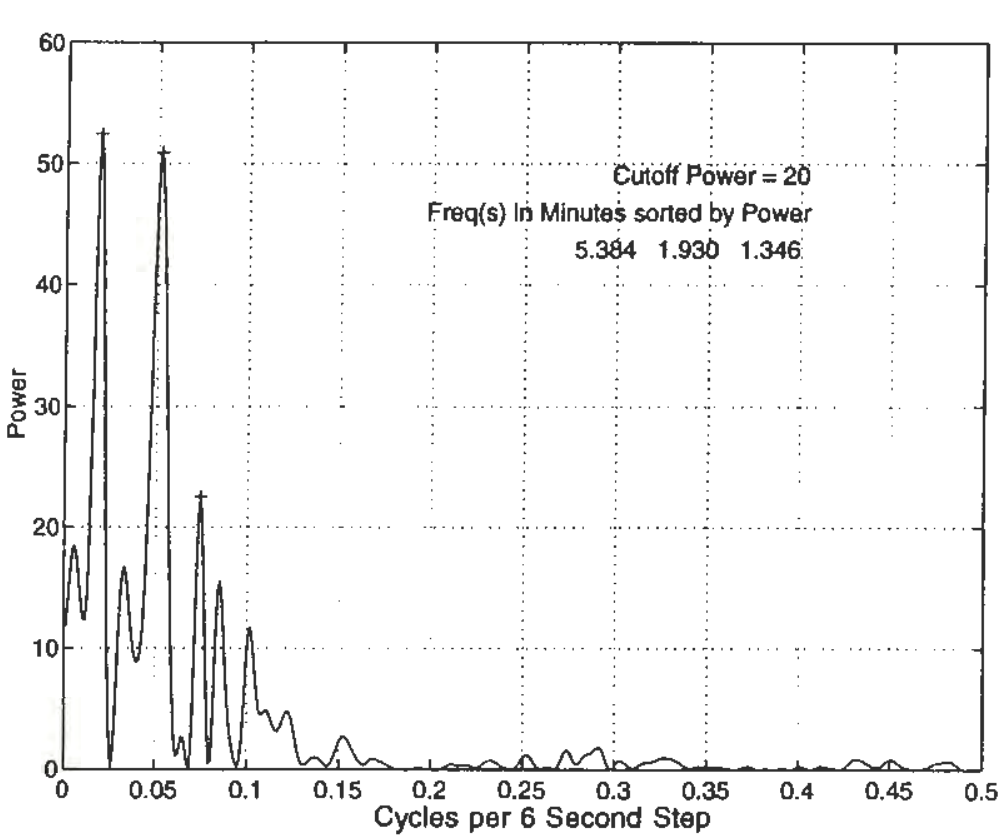

Average Reaction Time: 752.994

Reaction Time FFT

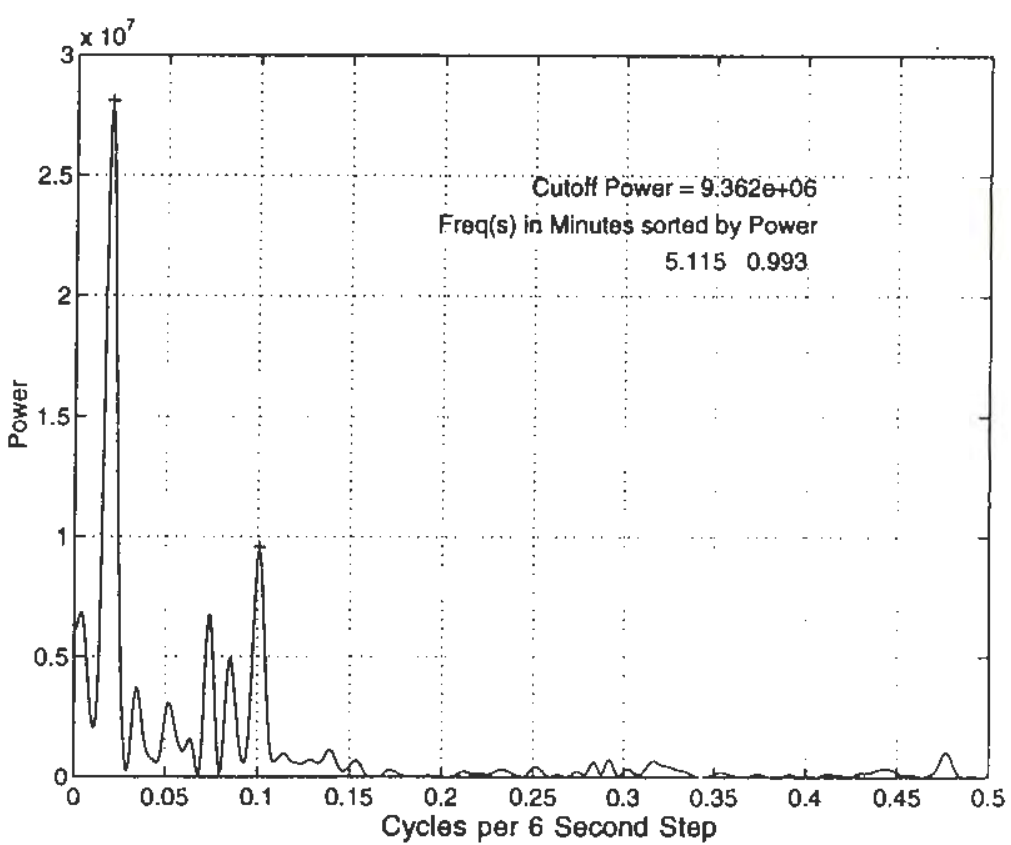




\section{Human Subject j038, Group 2}

Hit Rate: 0.773

Accuracy FFT

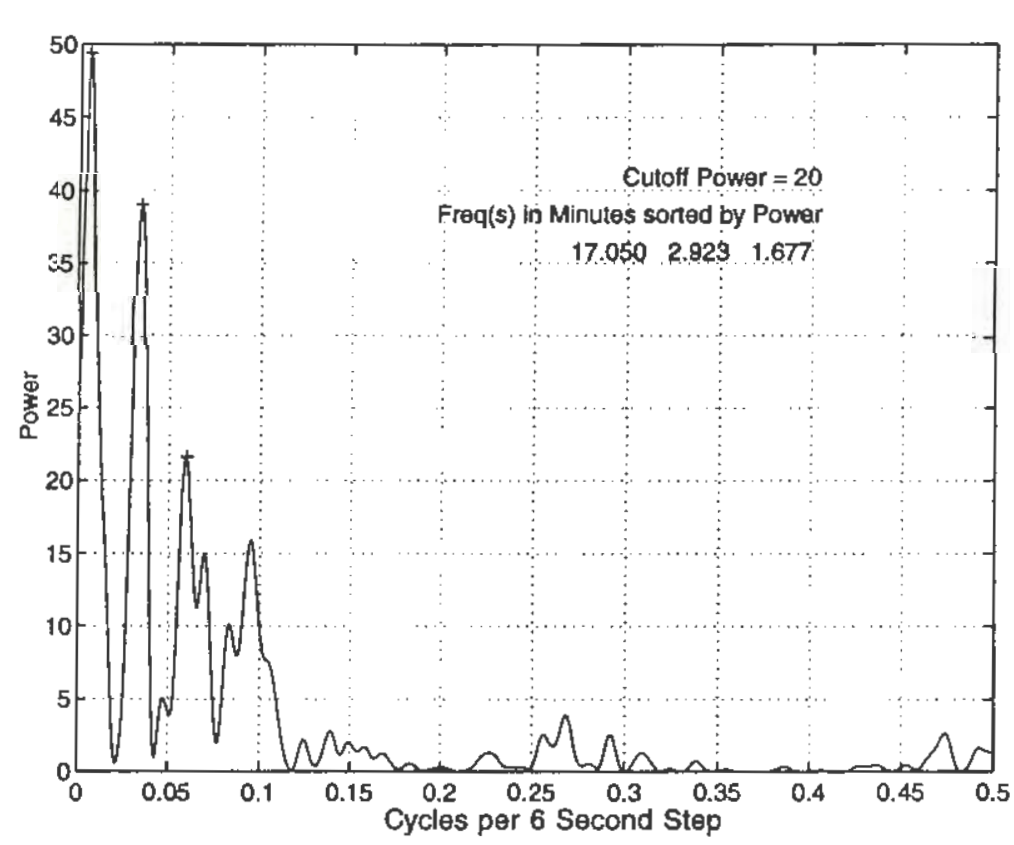

Average Reaction Time: 688.832

Reaction Time FFT

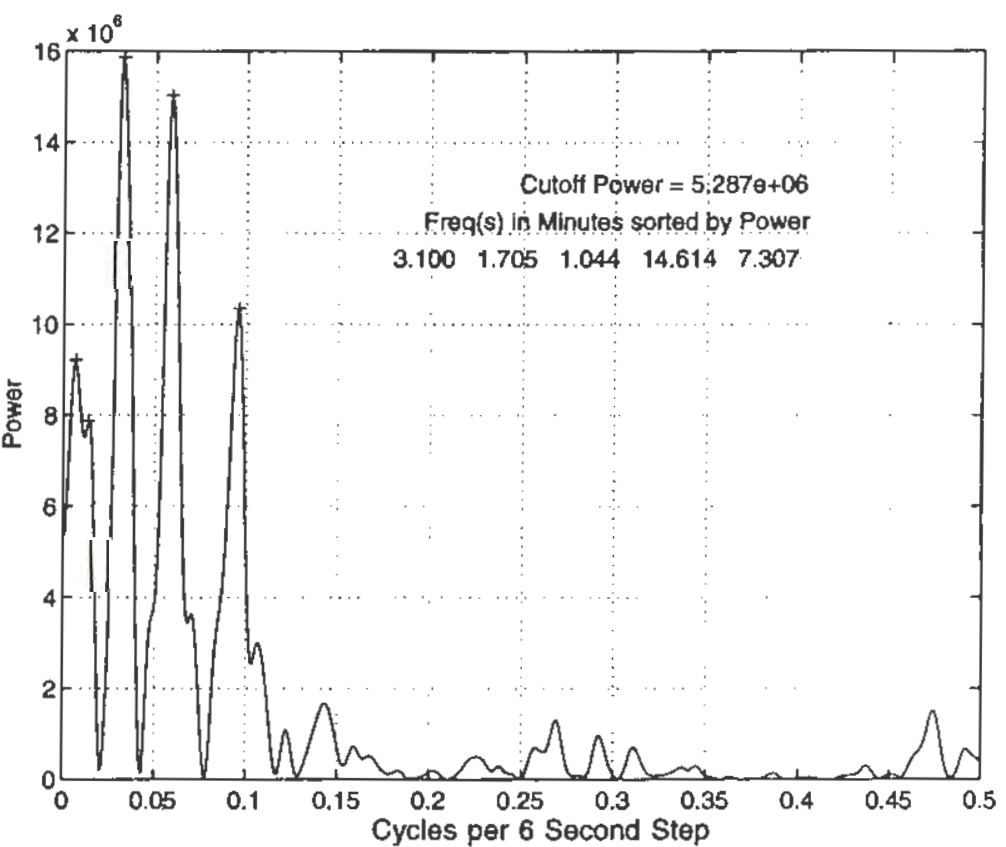




\section{Human Subject j039, Group 3}

Hit Rate: 0.832

\section{Accuracy FFT}

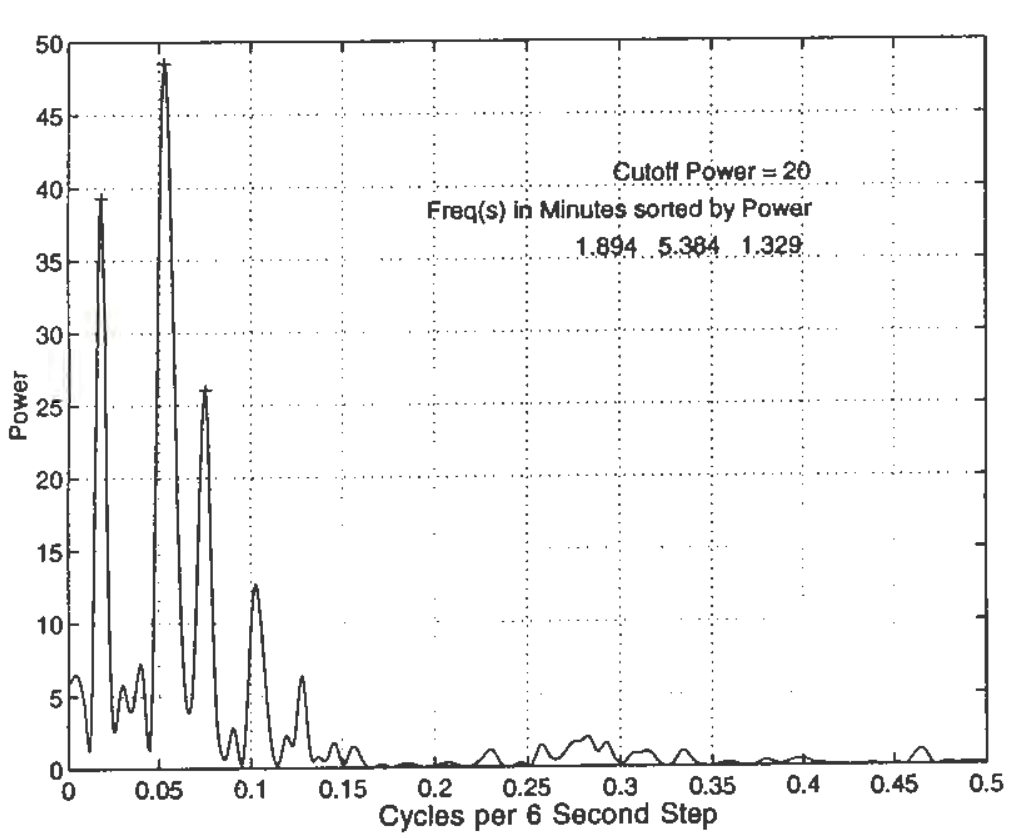

Average Reaction Time: 839.11

Reaction Time FFT

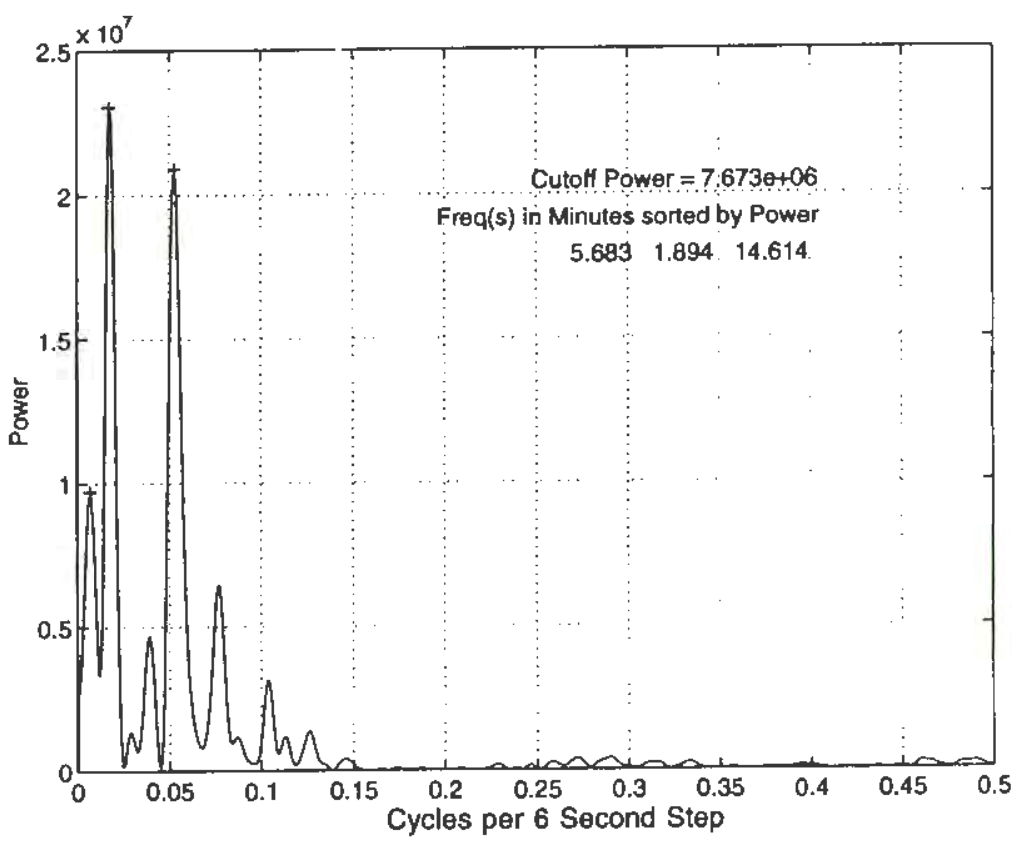




\section{Human Subject j040, Group 4}

Hit Rate: 0.897

Accuracy FFT

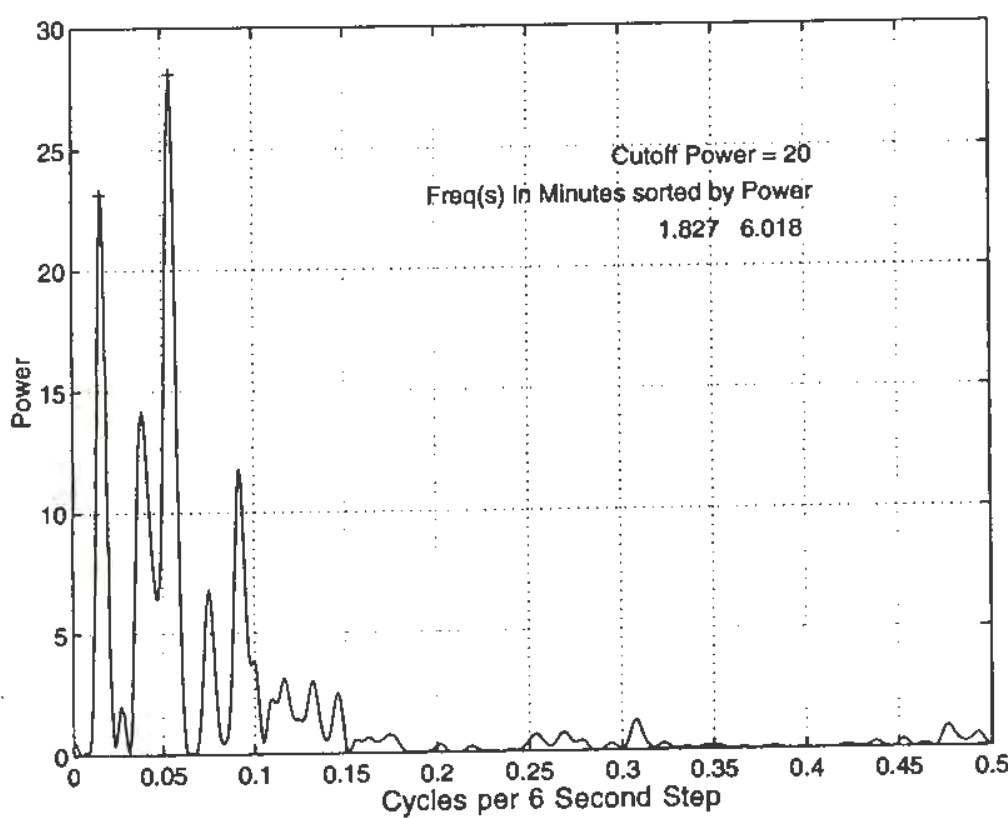

Average Reaction Time: 672.036

Reaction Time FFT

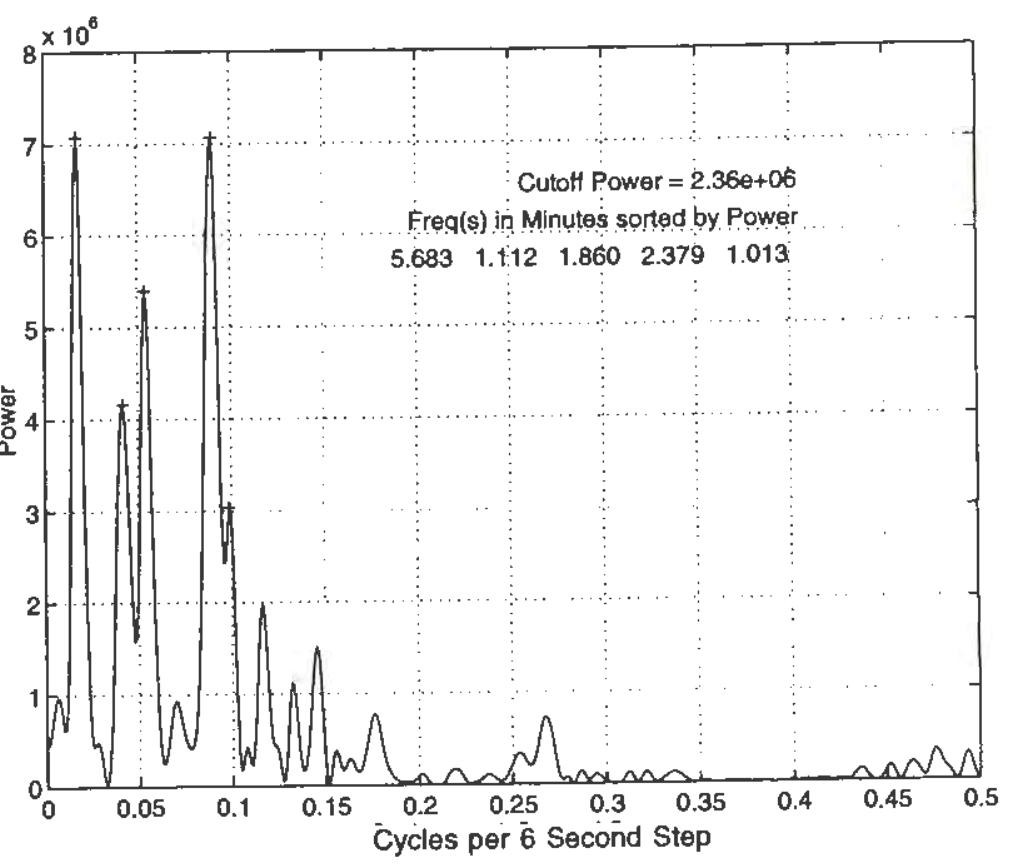




\section{Human Subject j042, Group 4}

Hit Rate: 0.935

Accuracy FFT

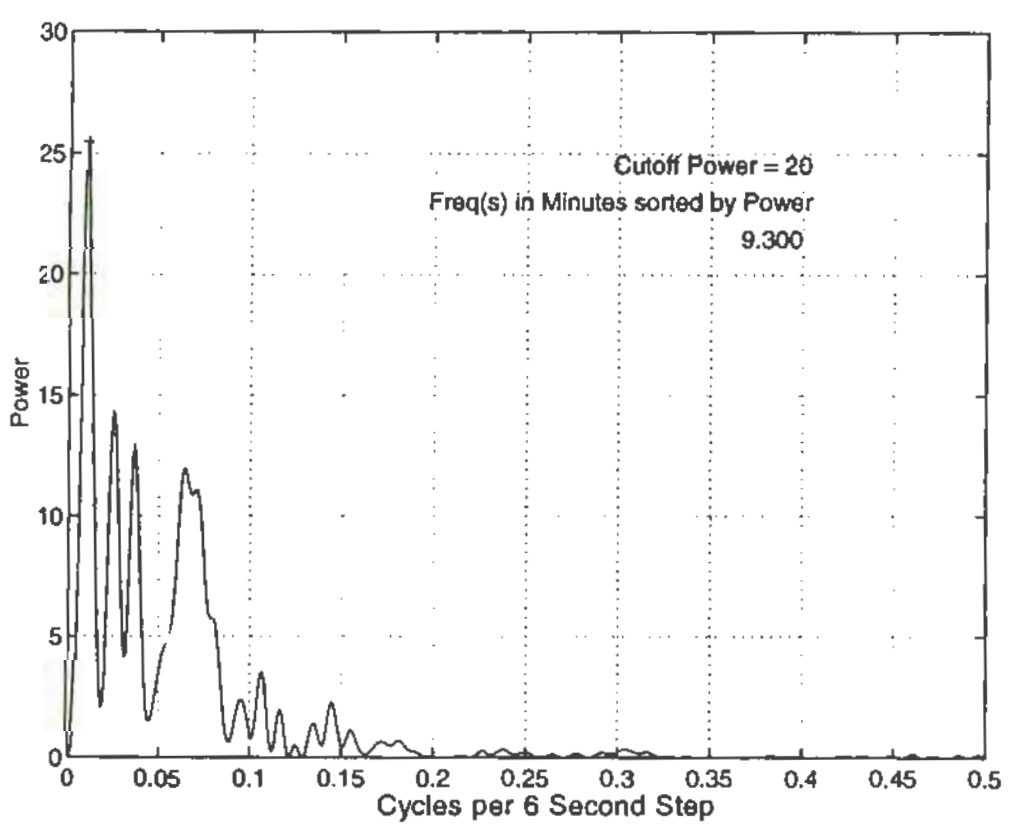

Average Reaction Time: 615.069

Reaction Time FFT

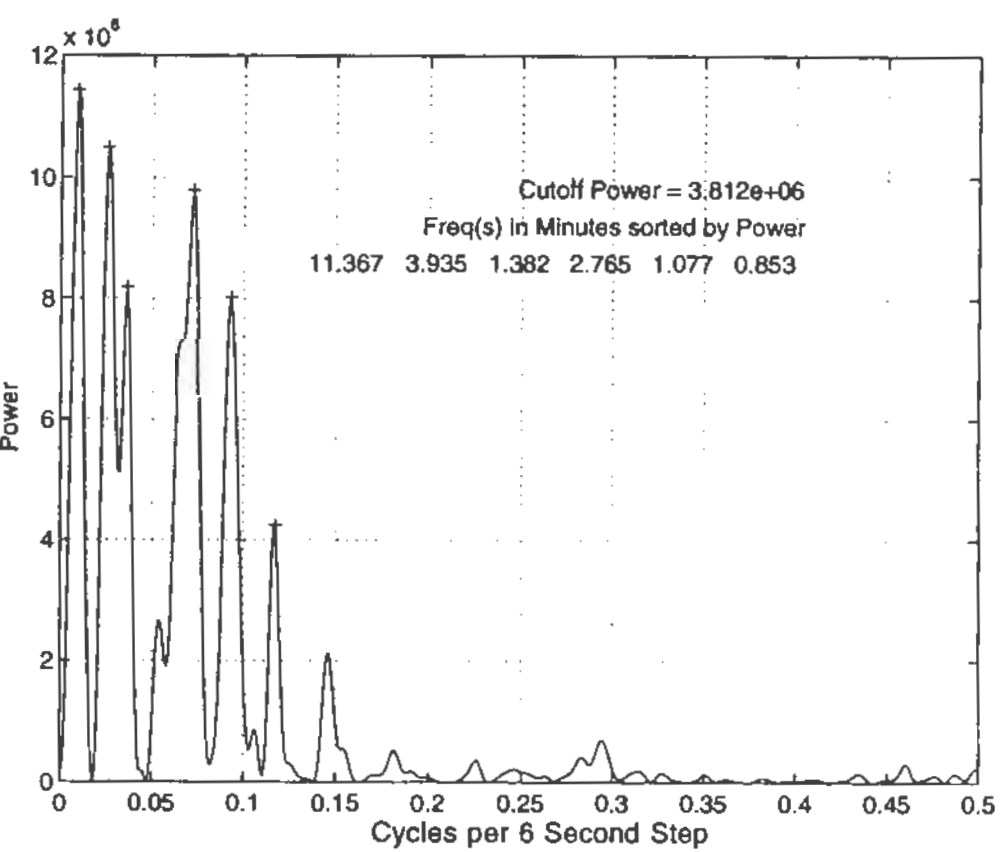




\section{Human Subject j043, Group 2}

Hit Rate: 0.757

Accuracy FFT

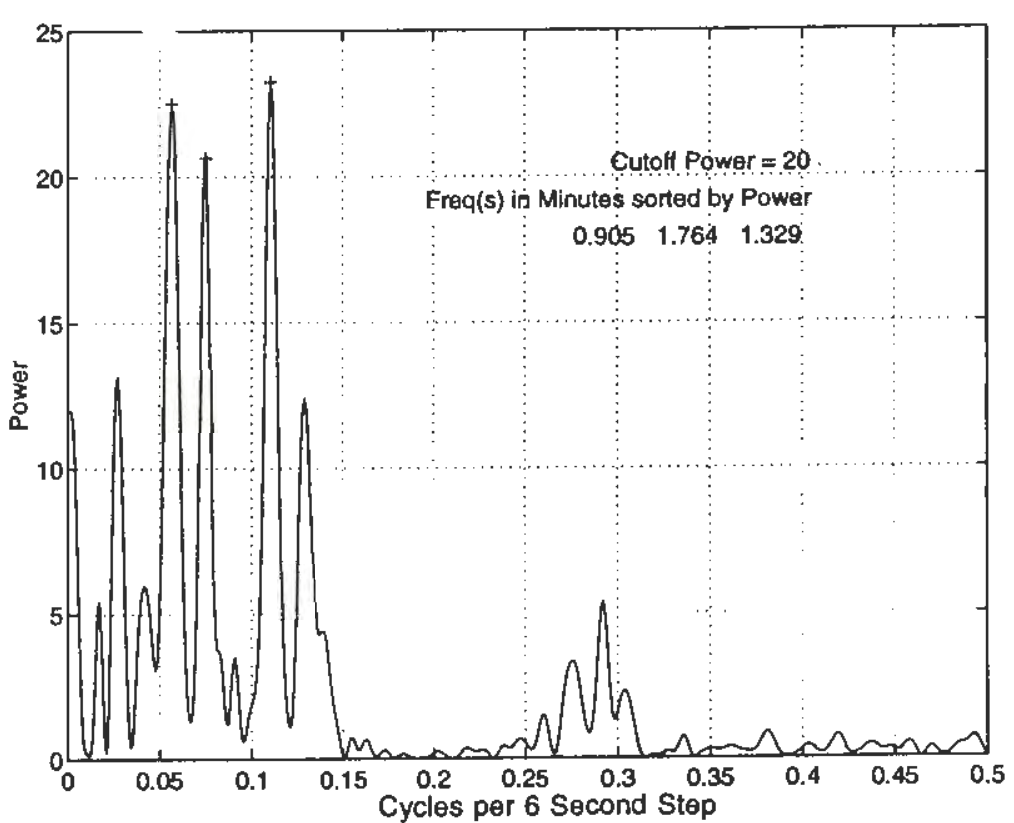

Average Reaction Time: 564.464

Reaction Time FFT

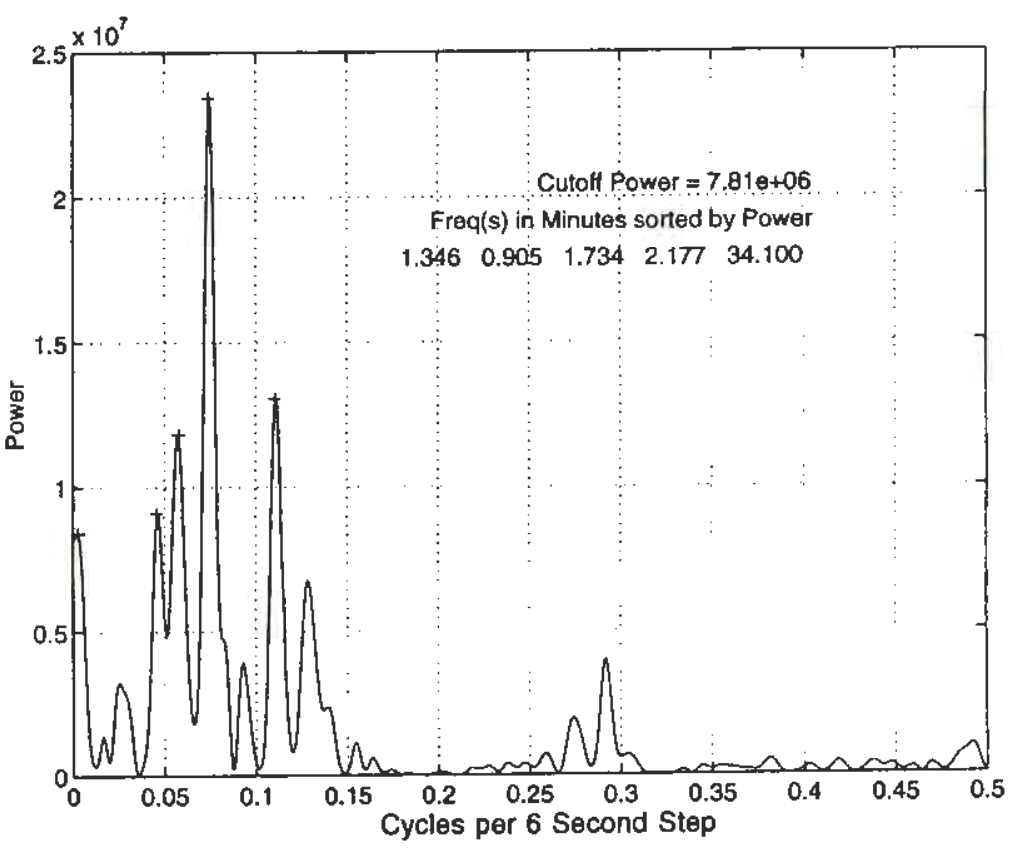




\section{Human Subject j045, Group 3}

Hit Rate: 0.811

Accuracy FFT

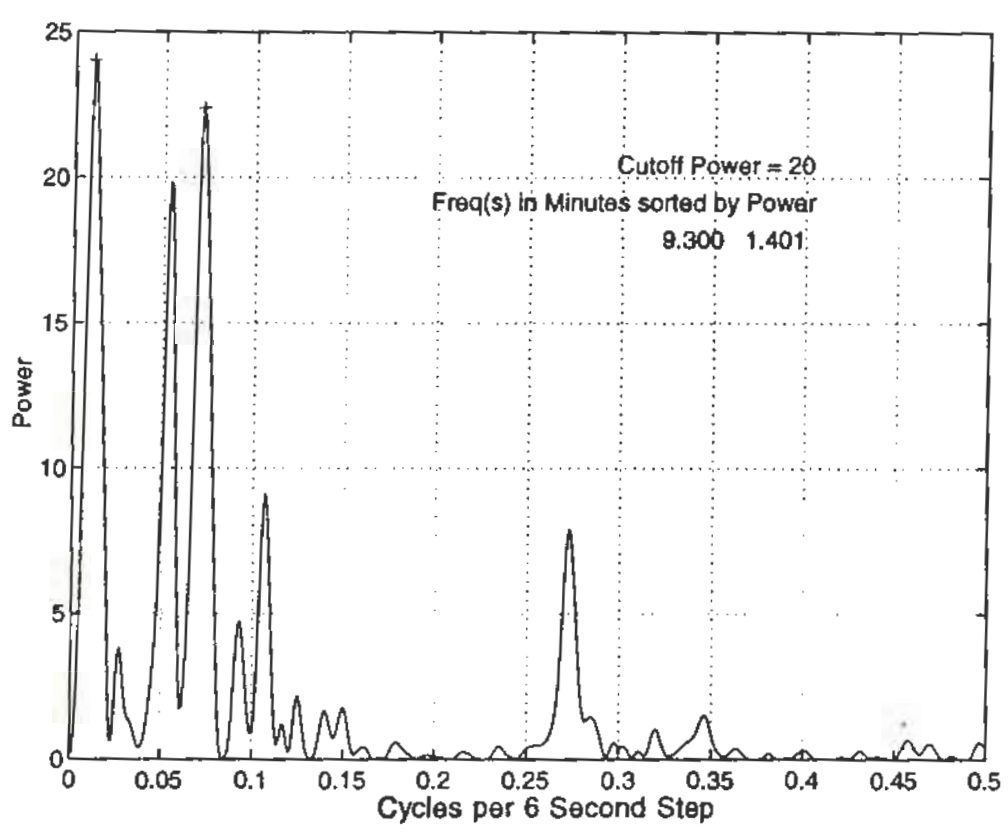

Average Reaction Time: 645.887

Reaction Time FFT

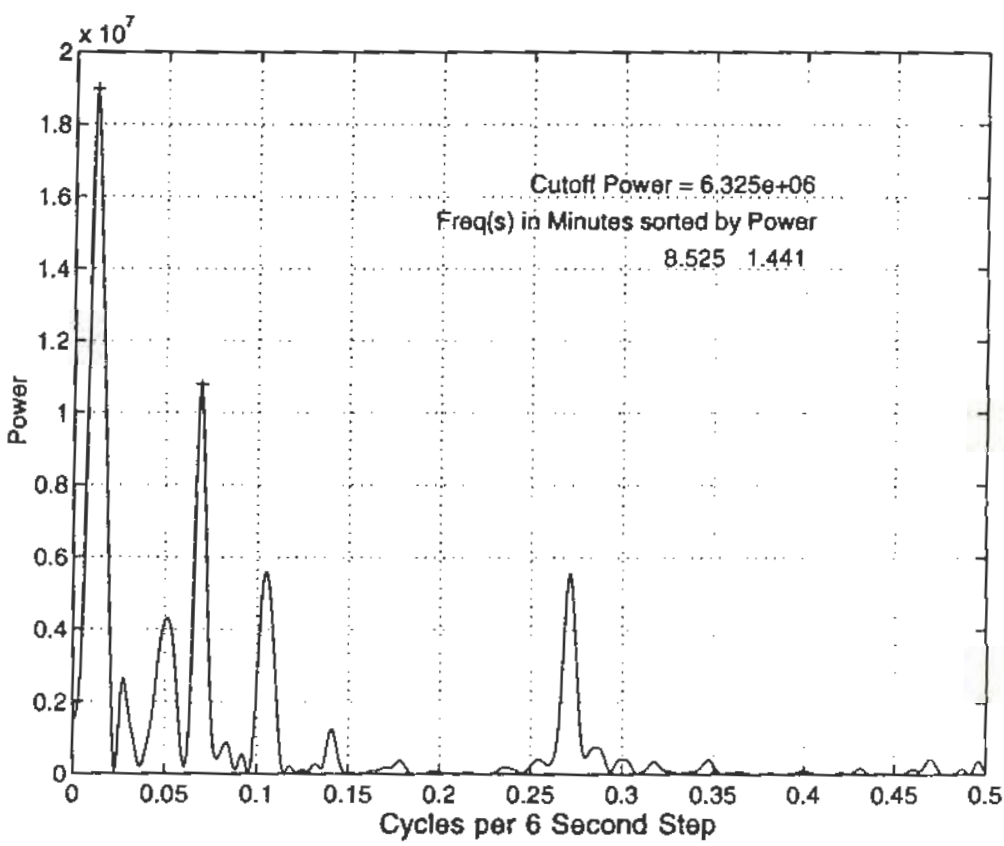




\section{Human Subject j046, Group 4}

Hit Rate: 0.876

Accuracy FFT

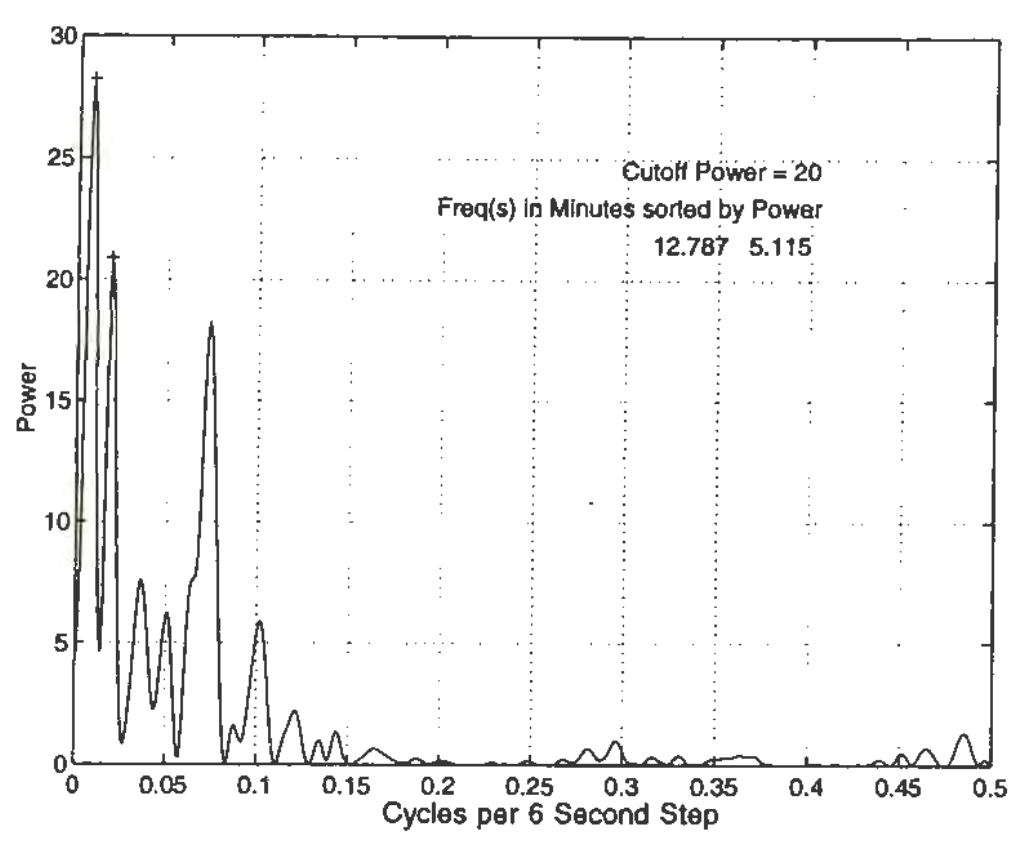

Average Reaction Time: 685.346

Reaction Time FFT

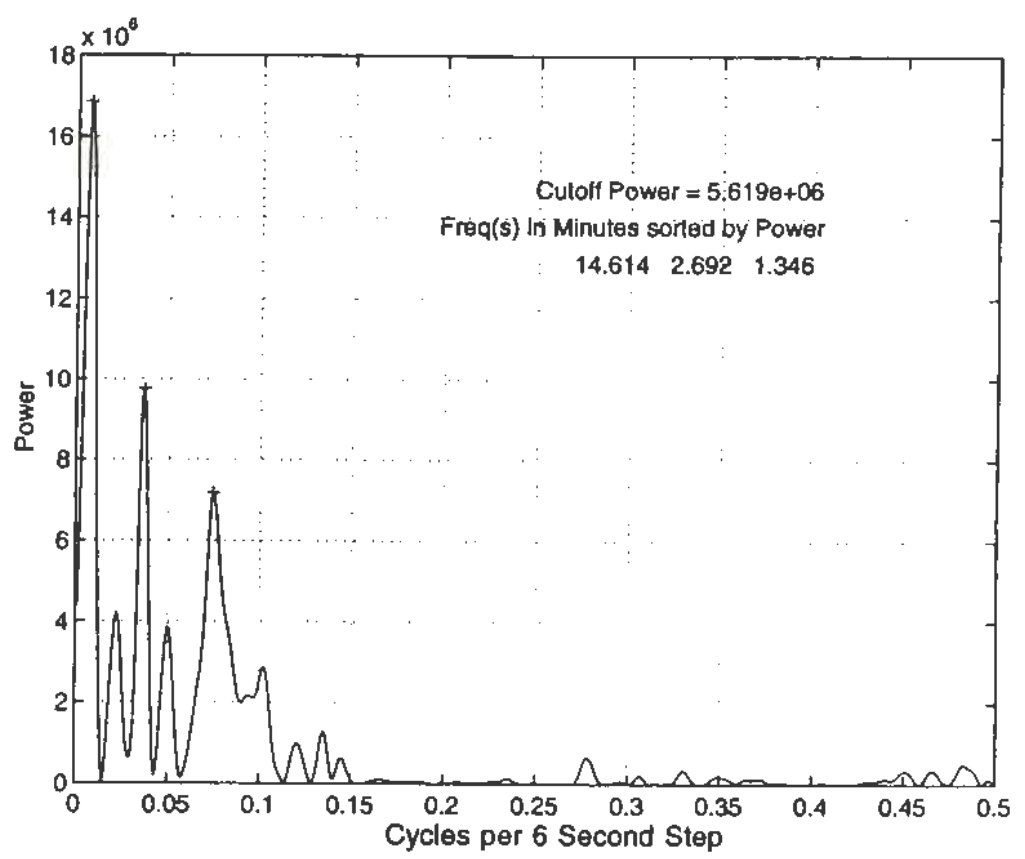




\section{Human Subject j047, Group 1}

Hit Rate: 0.643

Accuracy FFT

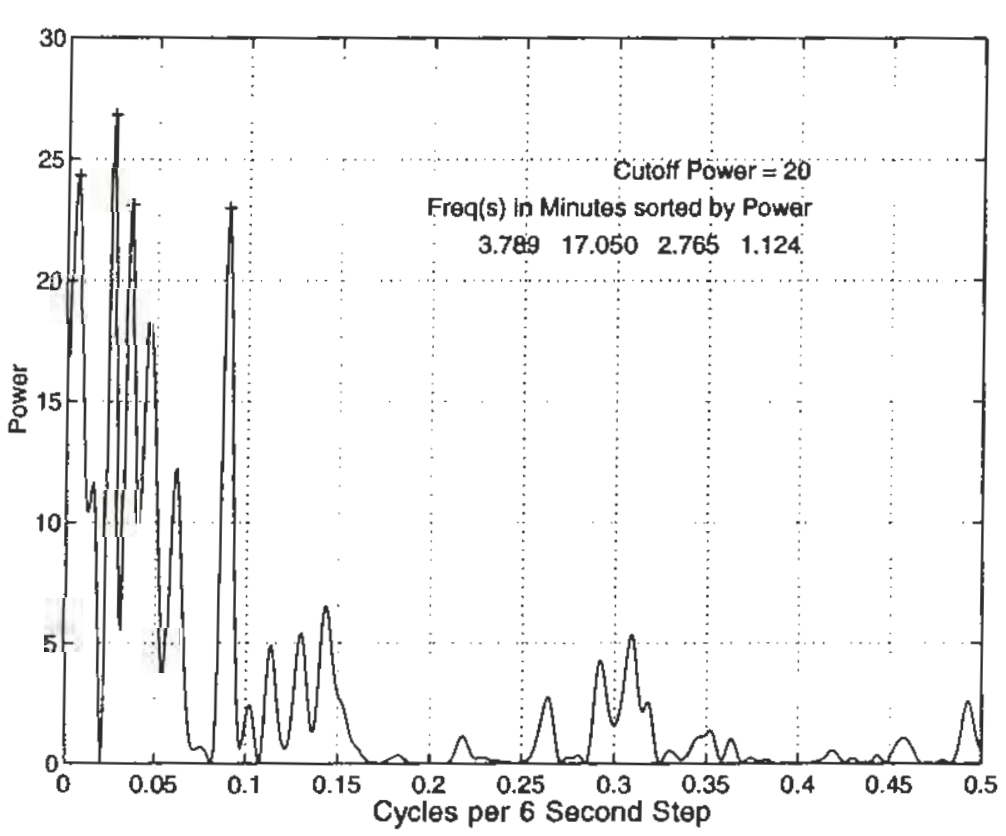

Average Reaction Time: 852.269

Reaction Time FFT

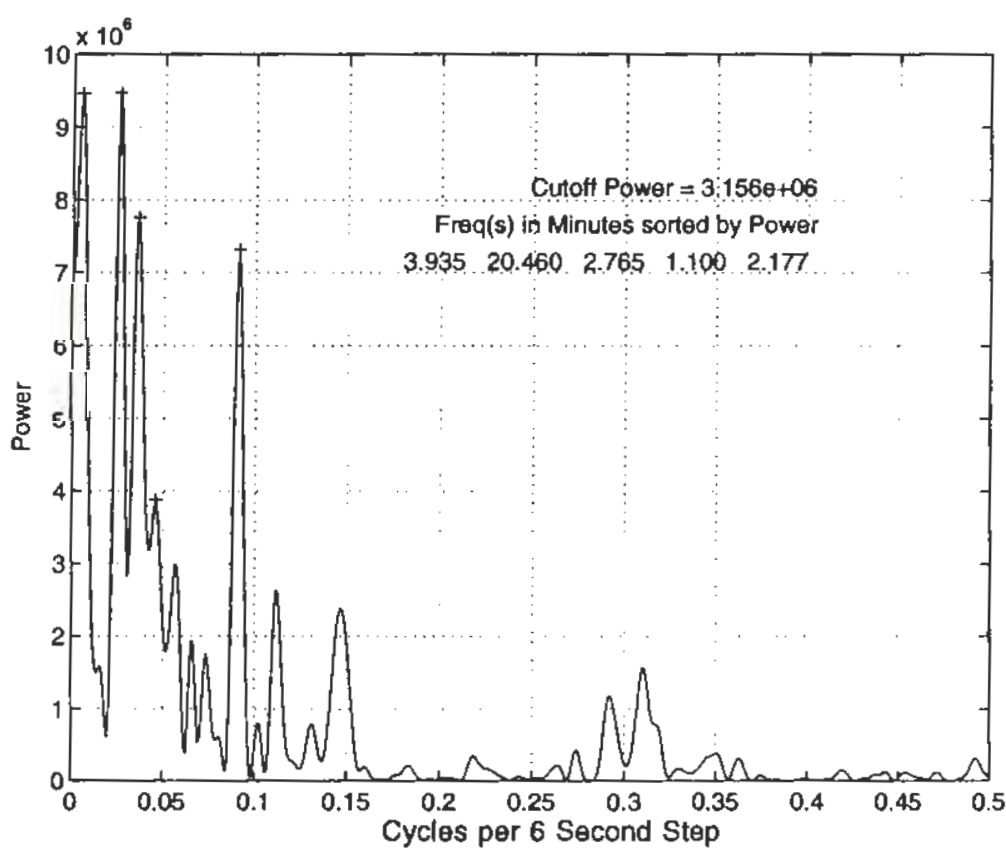




\section{Human Subject j048, Group 2}

Hit Rate: 0.773

Accuracy FFT

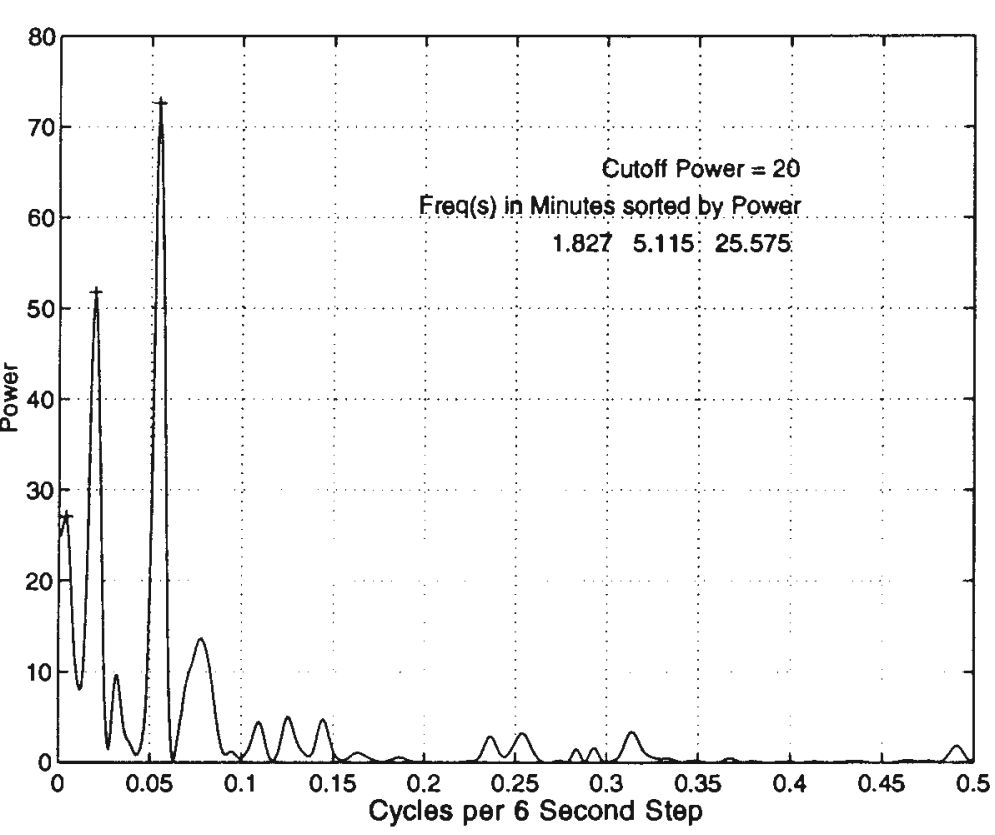

Average Reaction Time: 722.713

Reaction Time FFT

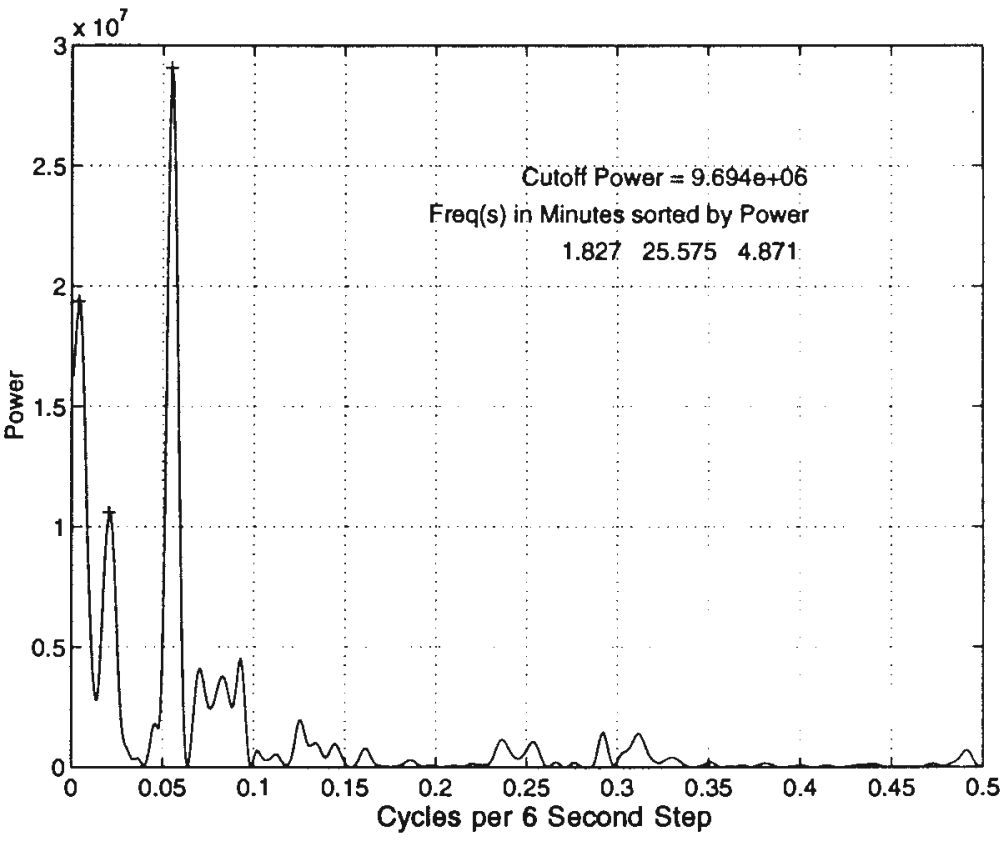




\section{Human Subject j049, Group 3}

Hit Rate: 0.87

Accuracy FFT

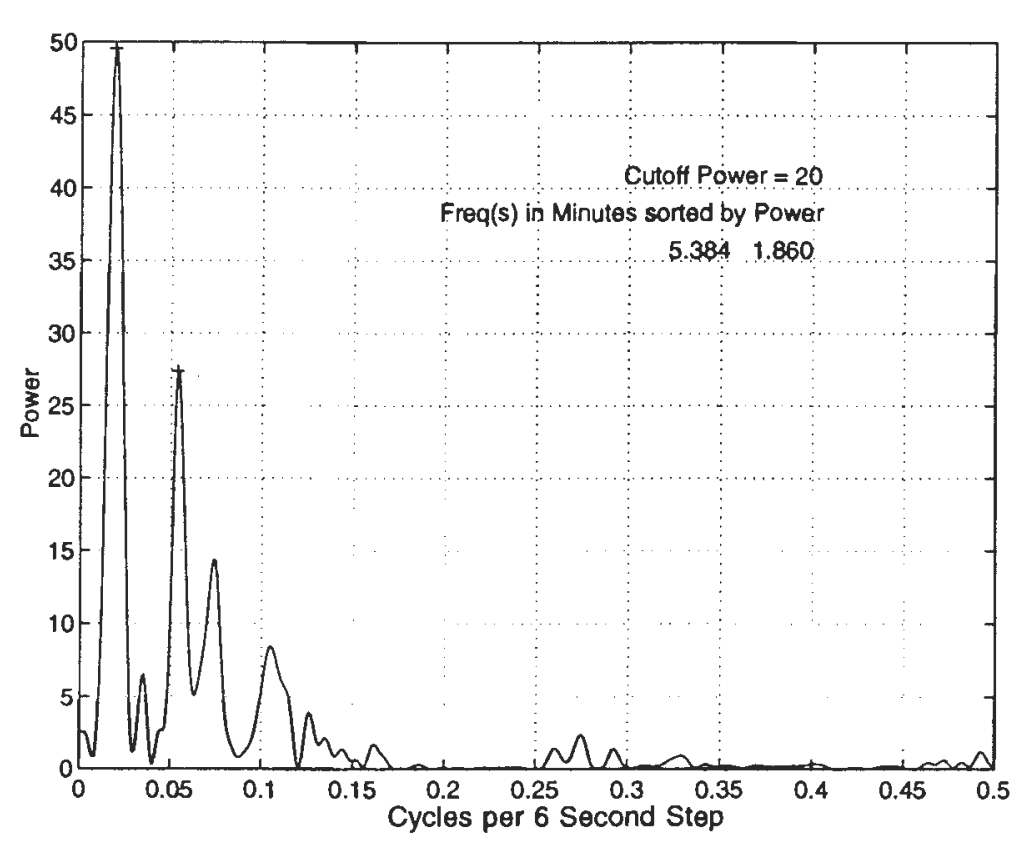

Average Reaction Time: 610.472

\section{Reaction Time FFT}

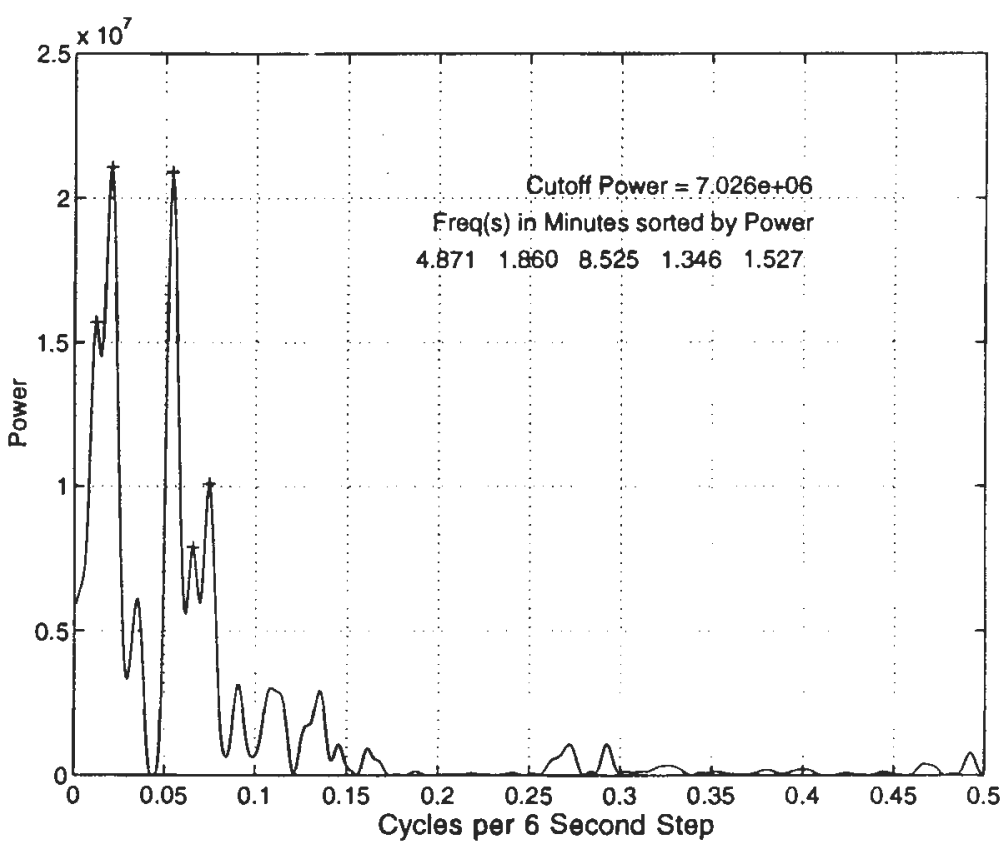




\section{Human Subject j050, Group 2}

Hit Rate: 0.741

Accuracy FFT

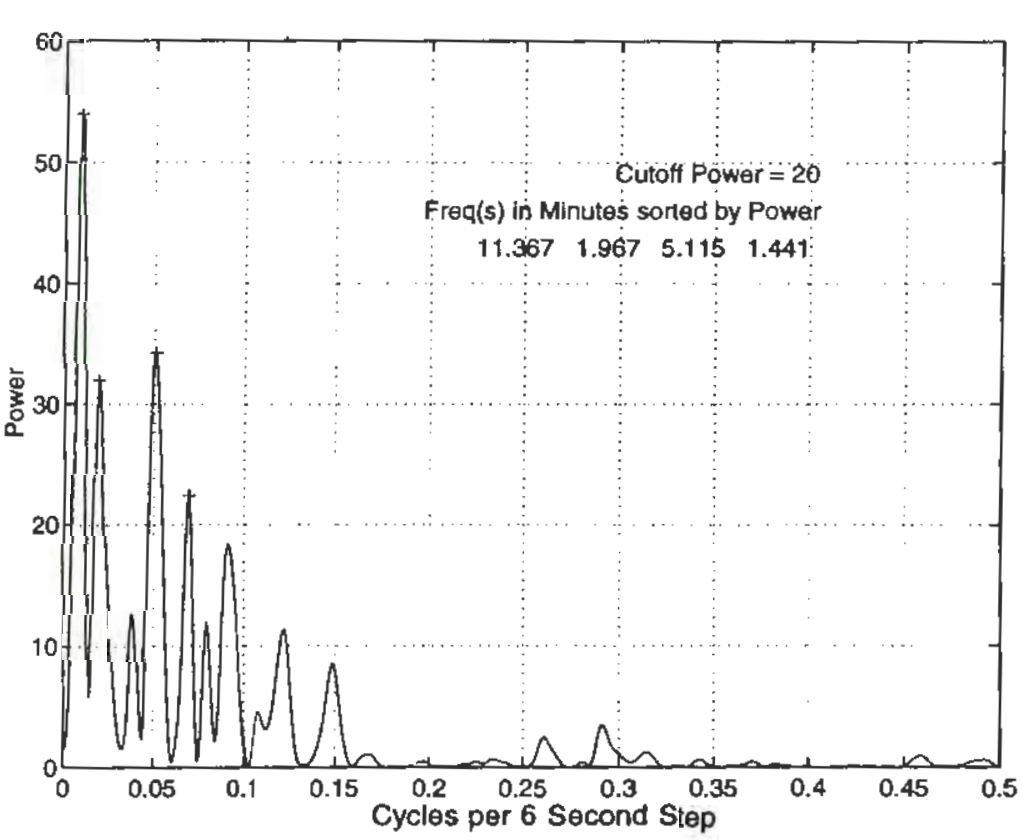

Average Reaction Time: 776.657

Reaction Time FFT

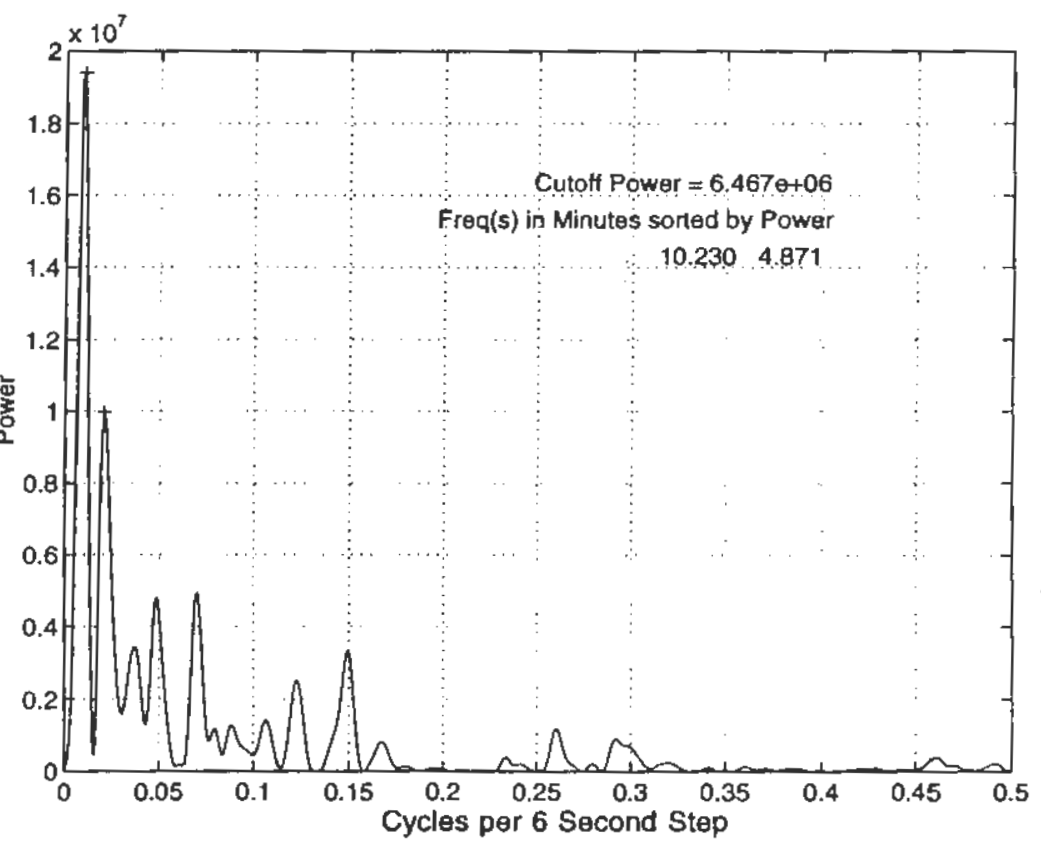




\section{Human Subject j051, Group 3}

Hit Rate: 0.822

Accuracy FFT

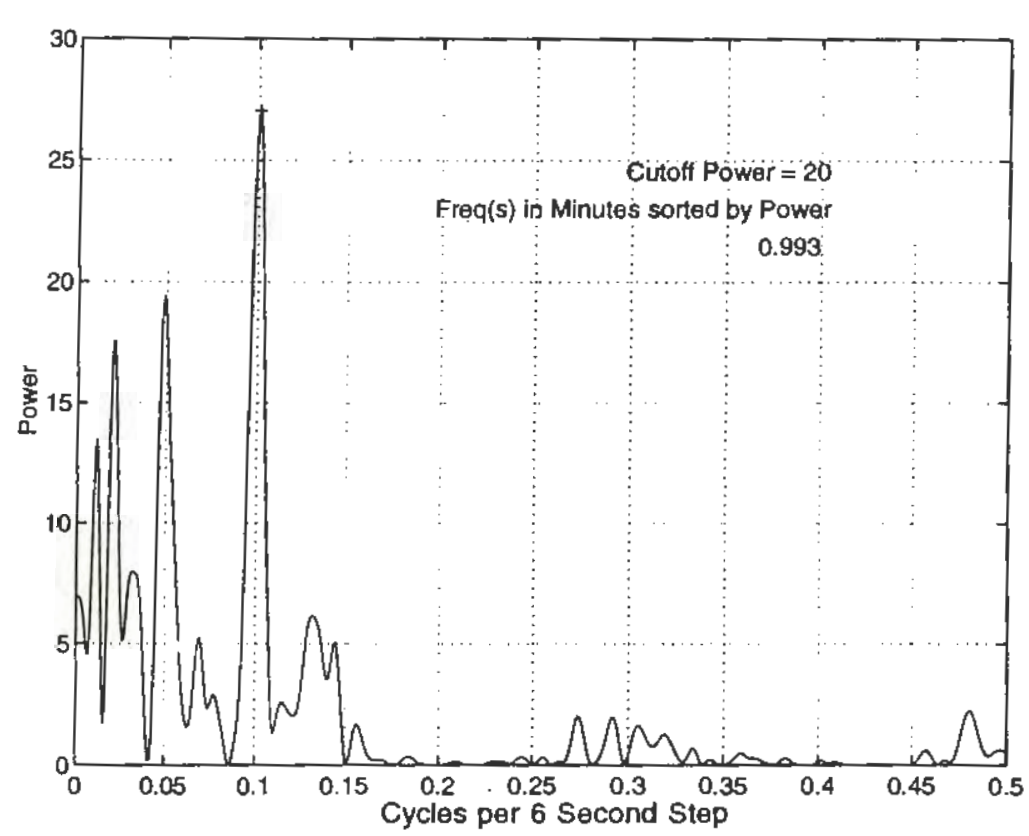

Average Reaction Time: 703.559

Reaction Time FFT

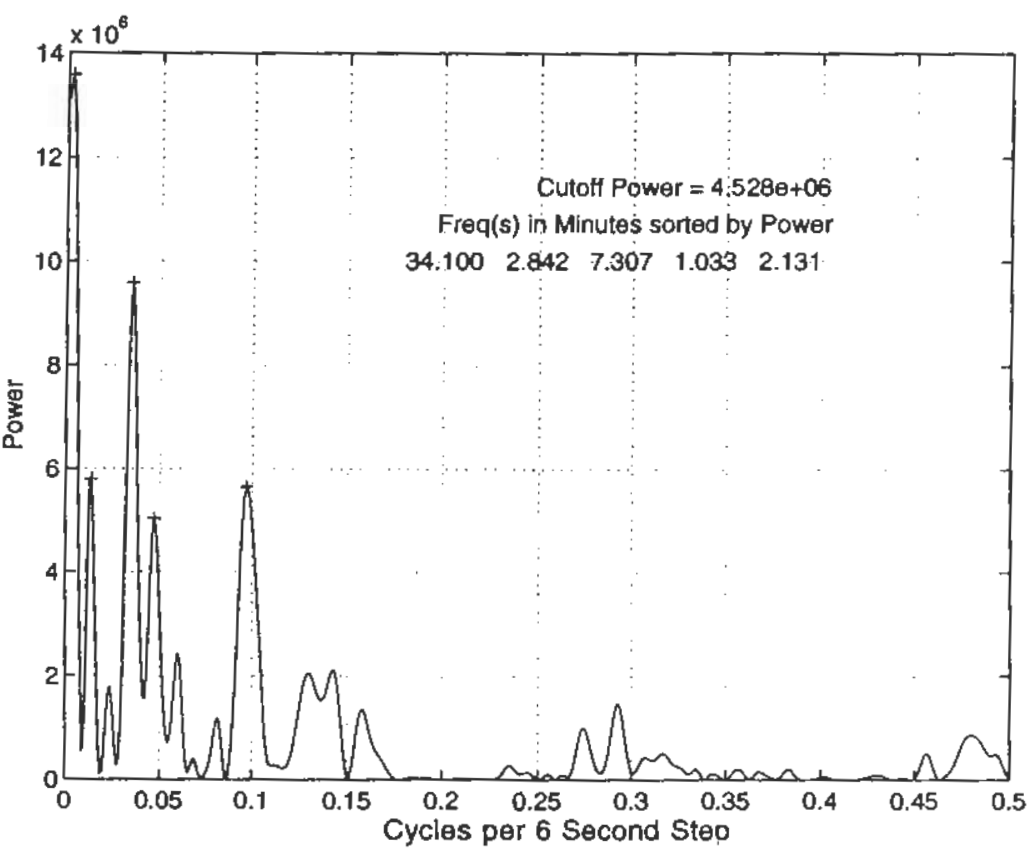




\section{Human Subject j052, Group 3}

Hit Rate: 0.854

Accuracy FFT

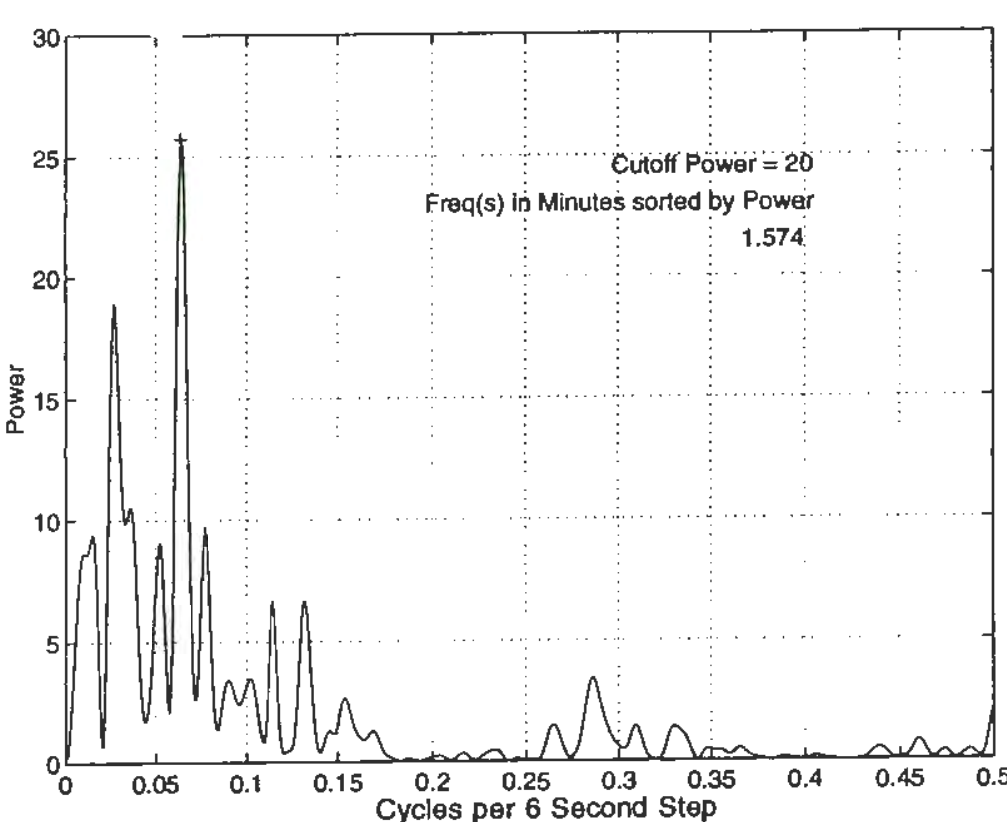

Average Reaction Time: 708.316

Reaction Time FFT

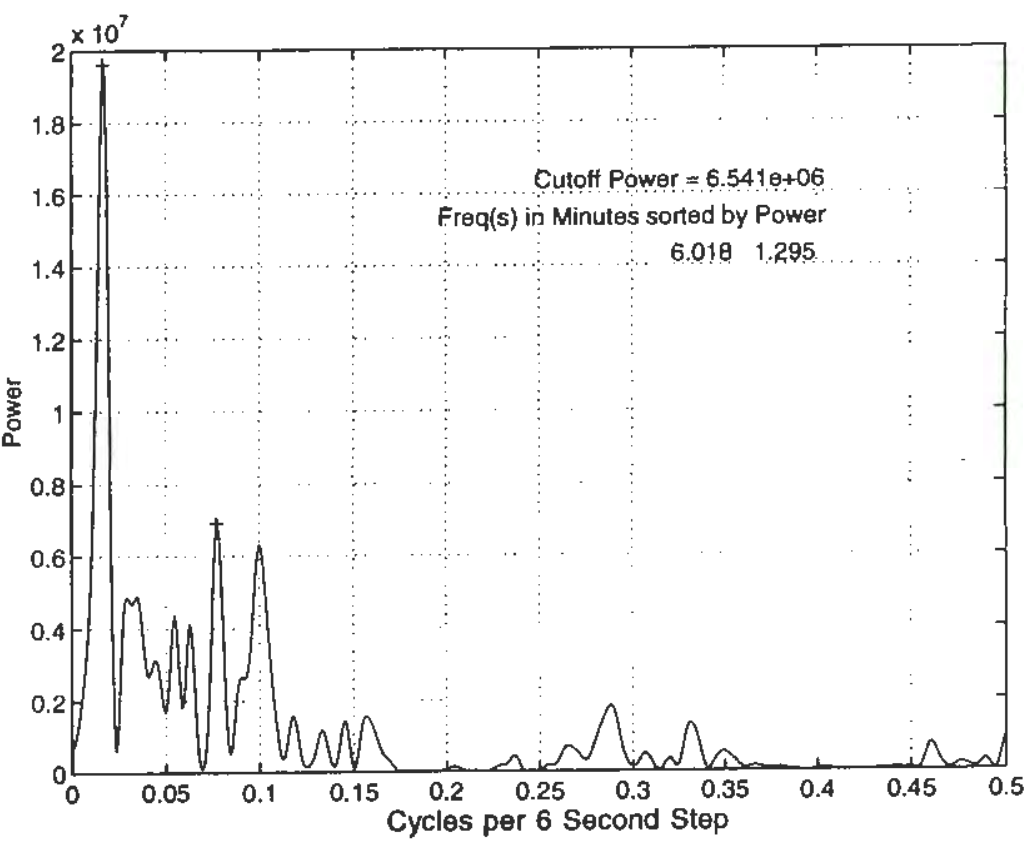




\section{Human Subject j054, Group 3}

Hit Rate: 0.843

Accuracy FFT

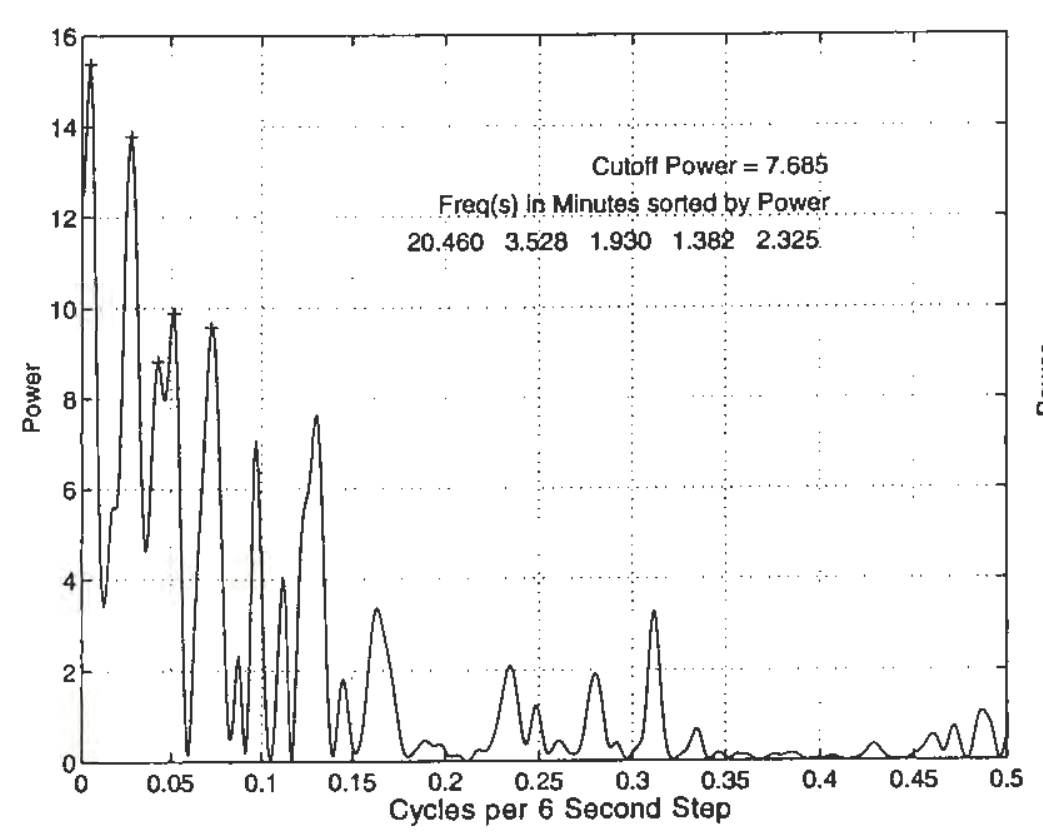

Average Reaction Time: 709.449

Reaction Time FFT

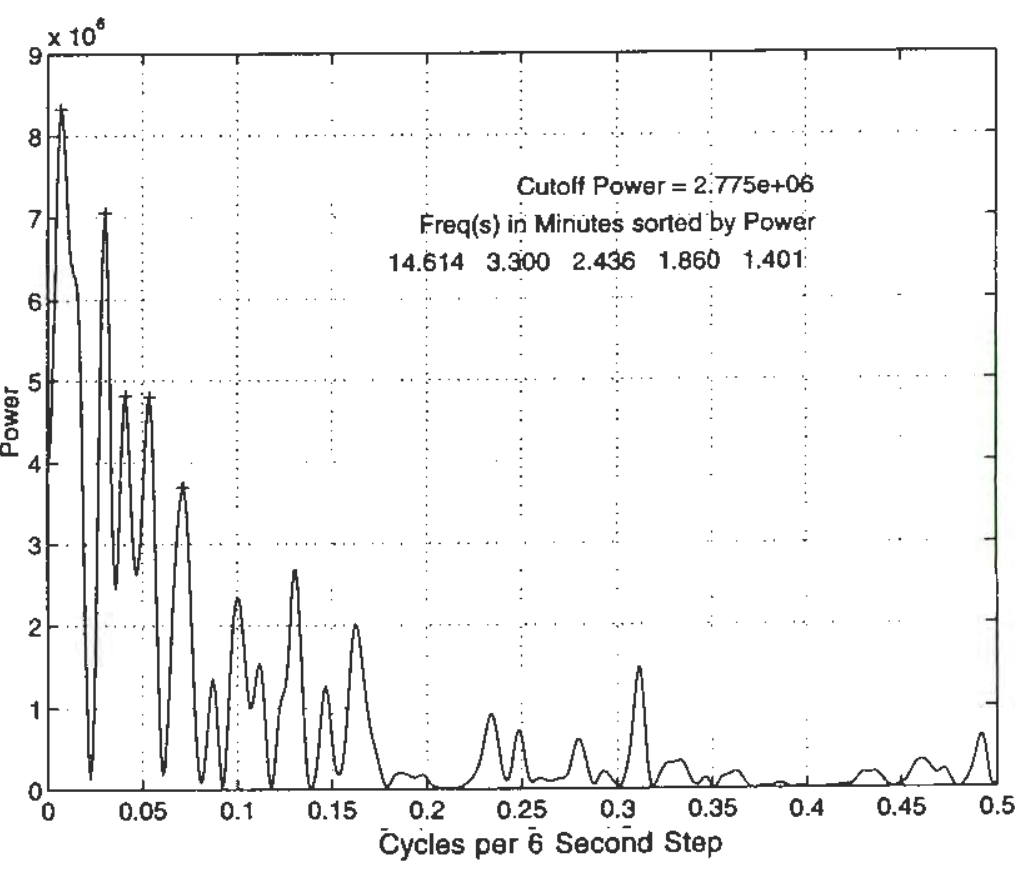




\section{Human Subject j055, Group 4}

Hit Rate: 0.881

Accuracy FFT

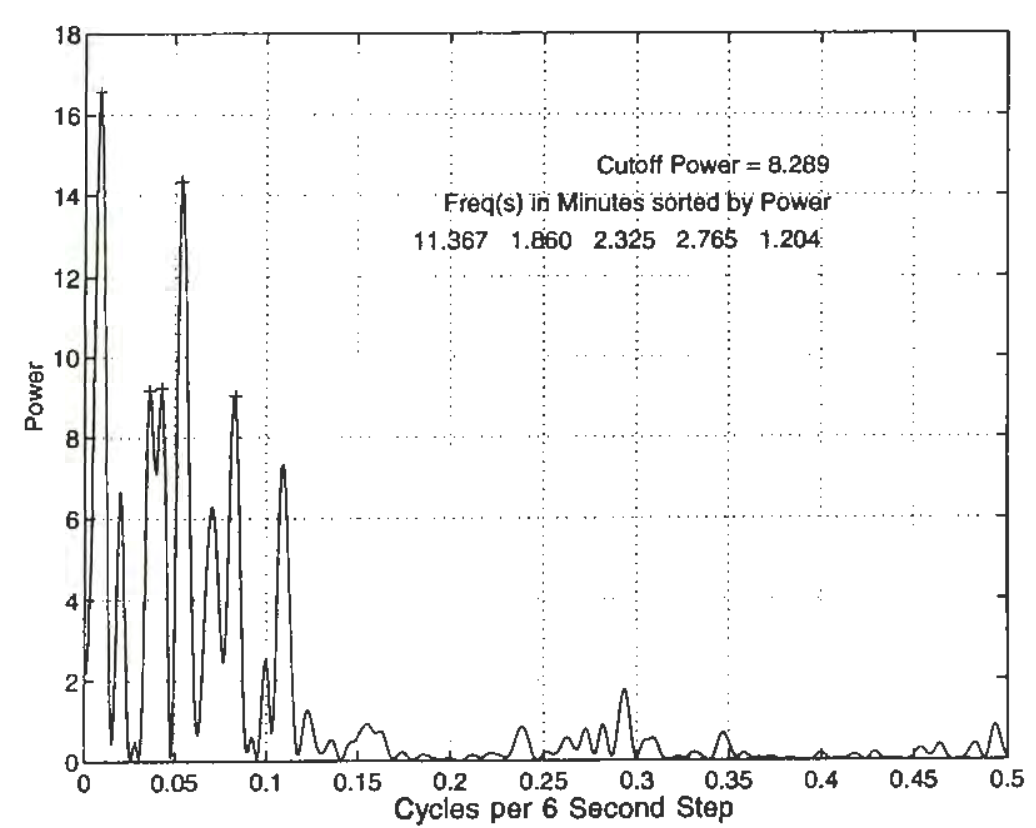

Average Reaction Time: 704.871

Reaction Time FFT

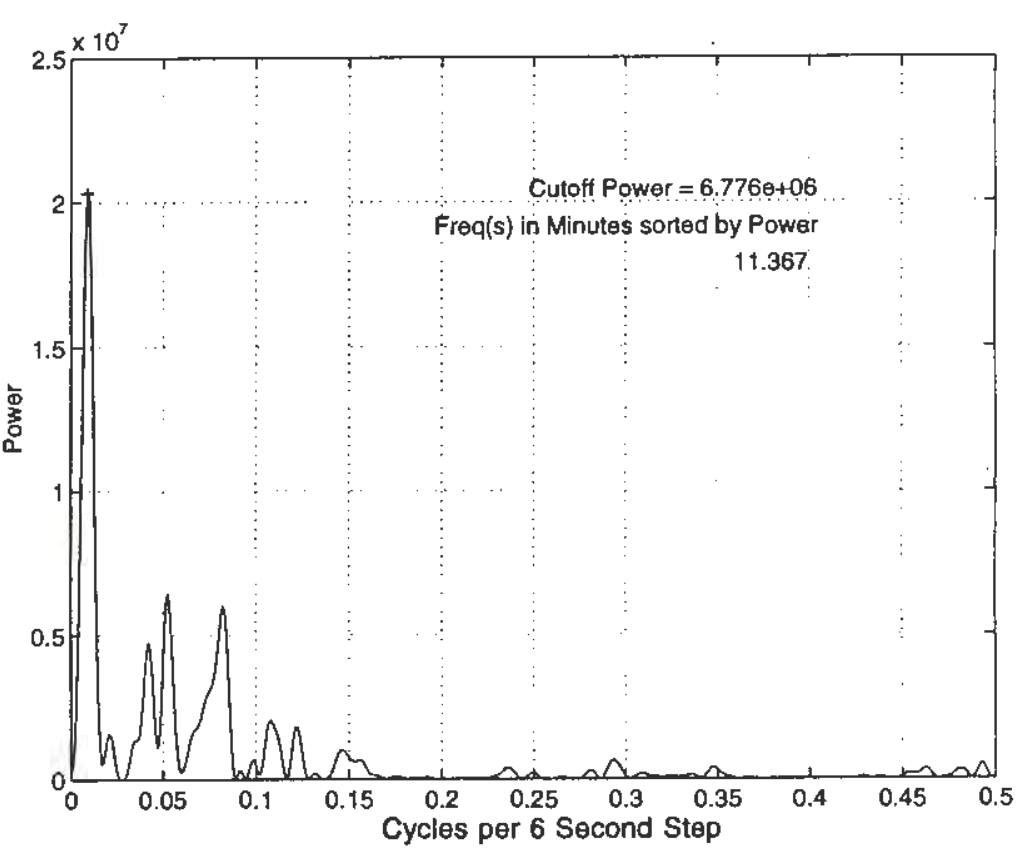




\section{Human Subject j056, Group 4}

Hit Rate: 0.935

Accuracy FFT

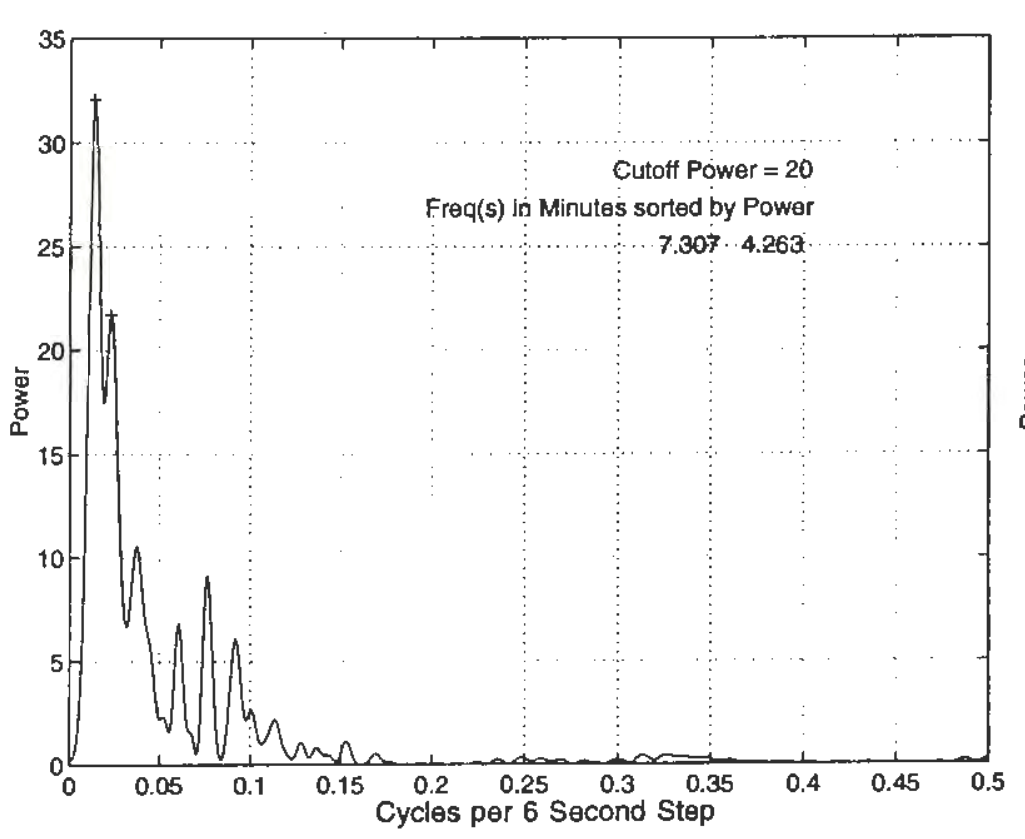

Average Reaction Time: 758.121

Reaction Time FFI

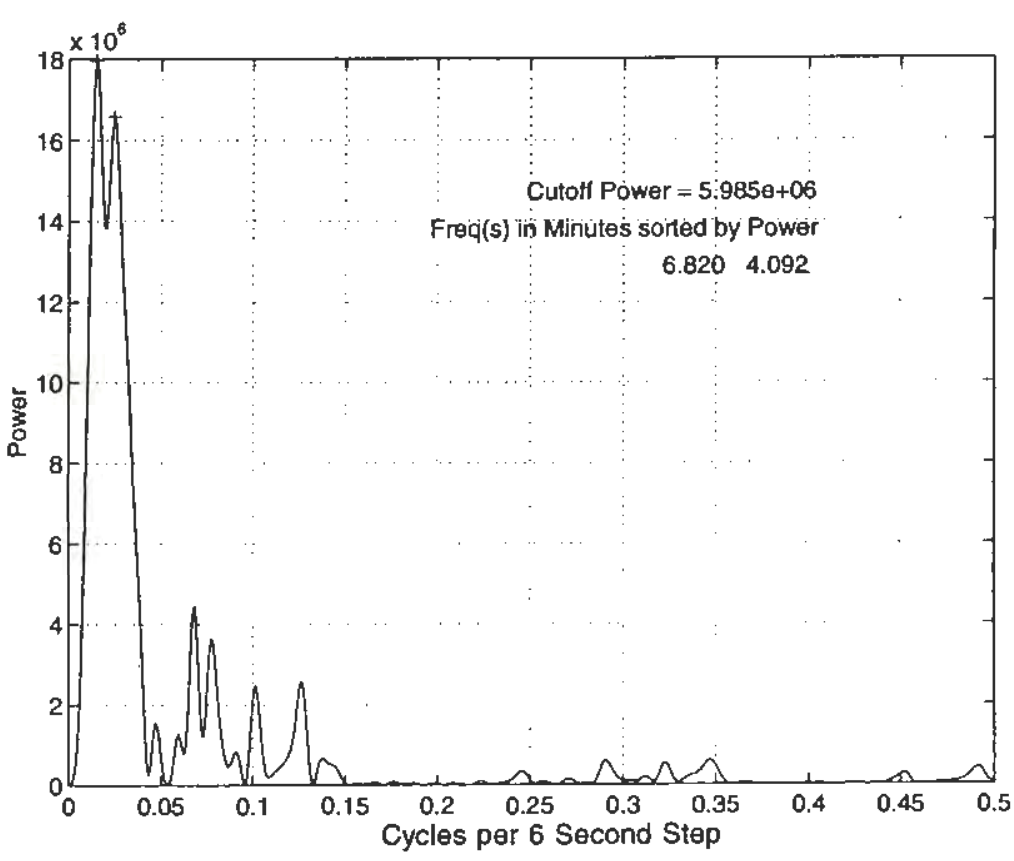




\section{Human Subject j057, Group 2}

Hit Rate: 0.773

Accuracy FFT

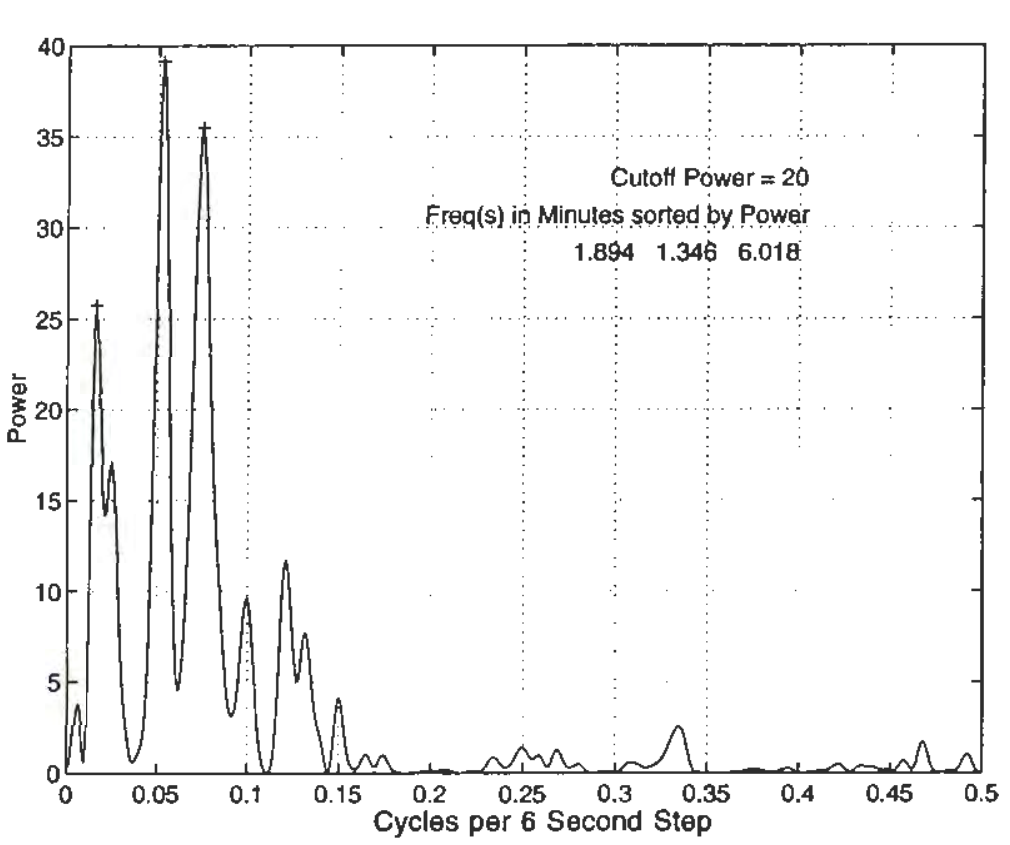

Average Reaction Time: 840.734

Reaction Time FFT

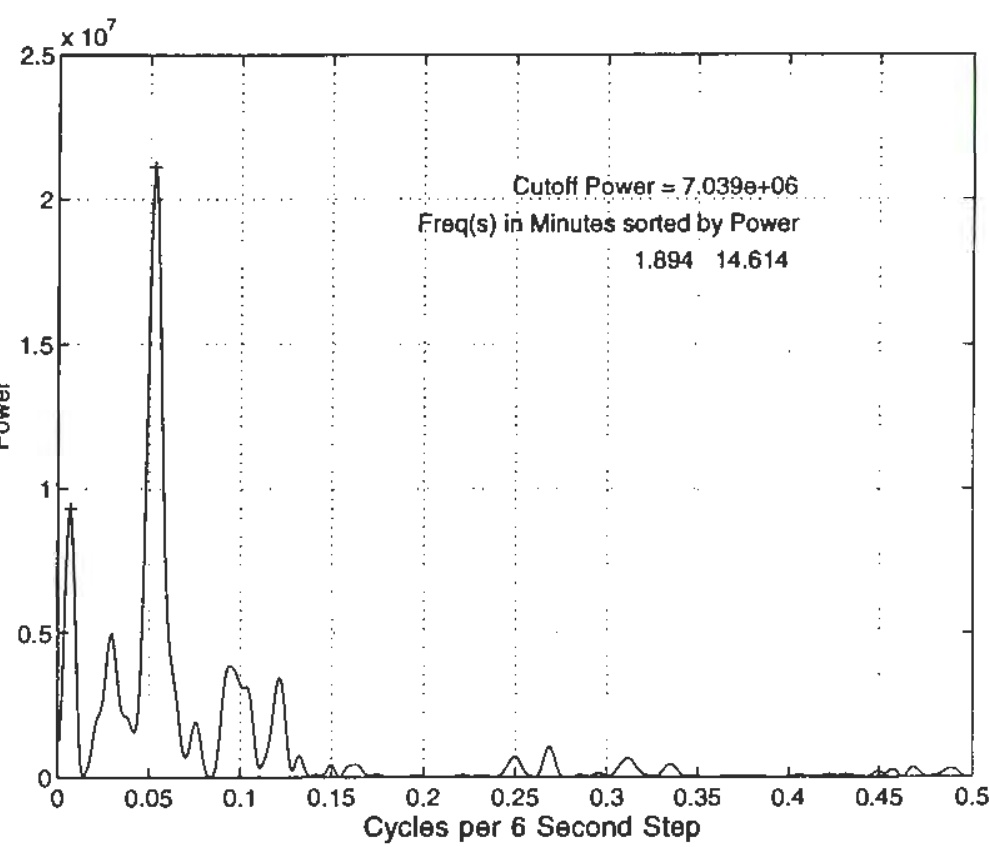




\section{Appendix M}

\section{Experiment 3 Part 3 Chi Squares}

Exp. 3 Part 3 Bin 1

\begin{tabular}{|c|c|c|c|c|c|c|c|}
\hline \multirow[b]{2}{*}{ Observed } & & G1 & $\mathrm{G} 2$ & G3 & \multicolumn{3}{|c|}{ G4 } \\
\hline & $\begin{array}{l}\text { fo }= \\
\text { fe= }\end{array}$ & $\begin{array}{c}8 \\
8.1 \\
\end{array}$ & $\begin{array}{l}\text { fo }=\quad 7 \\
\text { fe }=7.425\end{array}$ & $\begin{array}{lc}f o= & 6 \\
f e= & 6.075 \\
\end{array}$ & $\begin{array}{l}f o= \\
f e=\end{array}$ & $\begin{array}{c}6 \\
5.4 \\
\end{array}$ & 27 \\
\hline \multirow[t]{2}{*}{ Not Observed } & $\begin{array}{l}f o= \\
f e=\end{array}$ & $\begin{array}{c}4 \\
3.9 \\
\end{array}$ & $\begin{array}{ll}f o= & 4 \\
f e= & 3.575\end{array}$ & $\begin{array}{l}f o=3 \\
f e=2.925\end{array}$ & $\begin{array}{l}f o= \\
f e=\end{array}$ & $\begin{array}{c}2 \\
2.6\end{array}$ & 13 \\
\hline & & 12 & 11 & 9 & & 8 & 40 \\
\hline
\end{tabular}

$$
\begin{aligned}
\mathrm{X}^{2} \mathrm{obt} & =0.079 \quad \mathrm{X}^{2} \text { crit }=7.81 \\
\mathrm{df} & =3
\end{aligned}
$$

Exp. 3 Part $3 \quad$ Bin 2

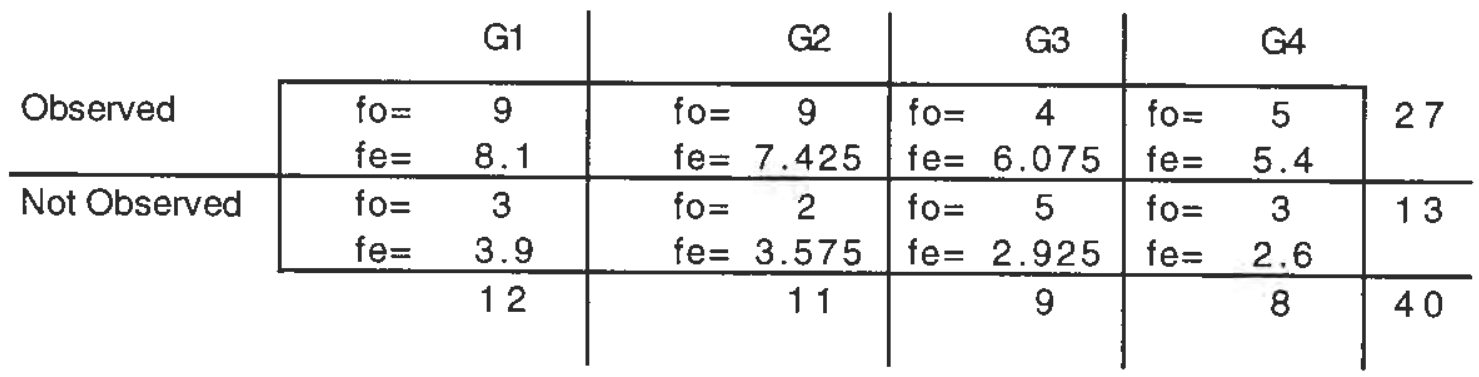

$$
\begin{aligned}
X^{2} \text { obt } & =1.336 \quad x^{2} \text { crit }=7.81 \\
d t & =3
\end{aligned}
$$


Exp. 3 Part $3 \quad$ Bin 3

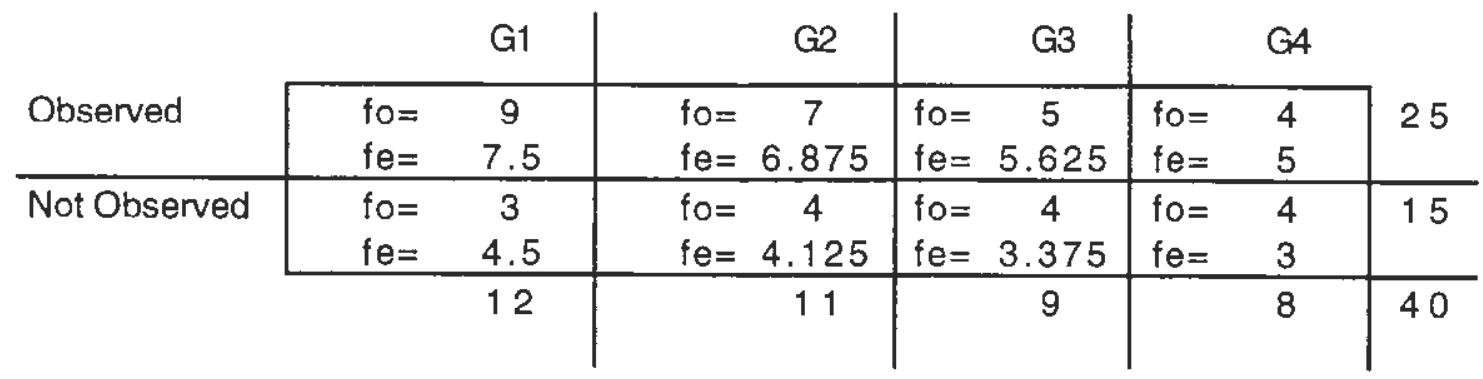

$$
\begin{aligned}
\mathrm{X}^{2} \mathrm{obt} & =0.806 \quad \mathrm{X}^{2} \mathrm{crit}=7.81 \\
\mathrm{df} & =3
\end{aligned}
$$

\begin{tabular}{|c|c|c|c|c|c|c|c|}
\hline \multirow[b]{2}{*}{ Observed } & \multicolumn{2}{|r|}{ G1 } & $\mathrm{G} 2$ & G3 & \multicolumn{3}{|c|}{ G4 } \\
\hline & $\begin{array}{l}f o= \\
f e=\end{array}$ & $\begin{array}{c}4 \\
6.9\end{array}$ & $\begin{array}{lc}f o= & 8 \\
f e= & 6.325\end{array}$ & $\begin{array}{lc}f o= & 7 \\
f e= & 5.175 \\
\end{array}$ & $\begin{array}{l}f o= \\
f e=\end{array}$ & $\begin{array}{c}4 \\
4.6\end{array}$ & 23 \\
\hline \multirow[t]{2}{*}{ Not Observed } & $\begin{array}{l}f o= \\
f e=\end{array}$ & $\begin{array}{c}8 \\
5.1\end{array}$ & $\begin{array}{l}f o=3 \\
f e=4.675\end{array}$ & $\begin{array}{l}f o=2 \\
f e=3.825\end{array}$ & $\begin{array}{l}f o= \\
f e=\end{array}$ & $\begin{array}{c}4 \\
3.4\end{array}$ & 17 \\
\hline & & 12 & 11 & 9 & & 8 & 40 \\
\hline
\end{tabular}

Exp. 3 Part $3 \quad$ Bin 4

$$
\begin{aligned}
X^{2} \text { obt } & =3.912 \quad X^{2} \text { crit }=7.81 \\
d f & =3
\end{aligned}
$$


Table 1

Subject Group Summary for for Experiment 1, Part 1

\begin{tabular}{lll}
\hline Hit Rate Range & $\underline{n}$ & Group Mean HR
\end{tabular}

\begin{tabular}{lccc}
\hline Group 1 & $58 \%-69 \%$ & 12 & $63 \%$ \\
Group 2 & $74 \%-80 \%$ & 11 & $77 \%$ \\
Group 3 & $81 \%-87 \%$ & 9 & $84 \%$ \\
Group 4 & $88 \%-94 \%$ & 8 & $90 \%$ \\
\hline
\end{tabular}


Table 2

Subject Group Summary for Experiment 1, Part 2

\begin{tabular}{lll}
\hline Hit Rate Range & $\underline{\mathrm{n}}$ & Group Mean HA
\end{tabular}

\begin{tabular}{llll}
\hline Group 1 & $58 \%-76 \%$ & 15 & $66 \%$ \\
Group 2 & $83 \%-94 \%$ & 15 & $88 \%$ \\
\hline
\end{tabular}


Table 3

Summary of Performance for all Subjects

\begin{tabular}{|c|c|c|c|c|c|c|}
\hline Subject & troup & HitRate & $\begin{array}{c}\text { \# of } \\
\text { False } \\
\text { Alarms }\end{array}$ & $\begin{array}{c}\text { \# of } \\
\text { Misses }\end{array}$ & $\begin{array}{l}\text { Hits after Hits } \\
\text { Ratio } \\
\text { Short to Long } \\
\text { Interval }\end{array}$ & $\begin{array}{c}\text { Misses after Hits } \\
\text { Ratio } \\
\text { Short to Long } \\
\text { Interval }\end{array}$ \\
\hline j009 & 1 & $69 \%$ & 12 & 57 & 1.171 & 2.545 \\
\hline j010 & 1 & $61 \%$ & 21 & 72 & 1.536 & 1.625 \\
\hline j011 & 1 & $58 \%$ & 11 & 77 & 1.296 & 2.833 \\
\hline j012 & 1 & $61 \%$ & 5 & 73 & 1.760 & 1.389 \\
\hline j013 & 1 & $62 \%$ & 7 & 71 & 0.971 & 1.250 \\
\hline j016 & 1 & $61 \%$ & 13 & 73 & 1.640 & 0.917 \\
\hline j017 & 1 & $63 \%$ & 8 & 68 & 1.536 & 1.556 \\
\hline j018 & 1 & $78 \%$ & 31 & 40 & 1.286 & 2.300 \\
\hline j020 & 1 & $67 \%$ & 26 & 62 & 1.341 & 1.250 \\
\hline j021 & 1 & $88 \%$ & 22 & 22 & 1.390 & 2.143 \\
\hline j022 & 1 & $80 \%$ & 20 & 38 & 1.521 & 1.000 \\
\hline j023 & 1 & $88 \%$ & 28 & 22 & 1.483 & 1.375 \\
\hline j024 & 2 & $76 \%$ & 25 & 44 & 1.455 & 2.300 \\
\hline j025 & 2 & $61 \%$ & 32 & 72 & 1.226 & 1.588 \\
\hline j026 & 2 & $84 \%$ & 38 & 30 & 1.263 & 3.333 \\
\hline j028 & 2 & $79 \%$ & 33 & 39 & 1.380 & 2.375 \\
\hline j030 & 2 & $78 \%$ & 25 & 41 & 1.675 & 1.467 \\
\hline j031 & 2 & $87 \%$ & 35 & 24 & 1.500 & 2.000 \\
\hline j032 & 2 & $74 \%$ & 42 & 48 & 1.208 & 2.100 \\
\hline j034 & 2 & $68 \%$ & 34 & 60 & 1.394 & 2.833 \\
\hline
\end{tabular}




$\begin{array}{lllllll}\text { j035 } & 2 & 67 \% & 34 & 61 & 1.342 & 1.500 \\ \text { j036 } & 2 & 89 \% & 41 & 20 & 1.426 & 3.250 \\ \text { j037 } & 2 & 85 \% & 42 & 27 & 1.210 & 6.000 \\ \text { j038 } & 3 & 77 \% & 46 & 42 & 1.523 & 2.556 \\ \text { j039 } & 3 & 83 \% & 25 & 31 & 1.345 & 2.571 \\ \text { j040 } & 3 & 90 \% & 22 & 19 & 1.586 & 2.200 \\ \text { j042 } & 3 & 94 \% & 34 & 12 & 1.629 & 0.800 \\ \text { j043 } & 3 & 76 \% & 14 & 45 & 1.429 & 3.111 \\ \text { j045 } & 3 & 81 \% & 13 & 35 & 1.429 & 1.583 \\ \text { j046 } & 3 & 88 \% & 24 & 23 & 1.258 & 6.333 \\ \text { j047 } & 3 & 64 \% & 36 & 66 & 1.357 & 1.789 \\ \text { j048 } & 3 & 77 \% & 40 & 42 & 1.313 & 2.556 \\ \text { j049 } & 4 & 87 \% & 32 & 24 & 1.545 & 1.333 \\ \text { j050 } & 4 & 74 \% & 34 & 48 & 1.512 & 1.429 \\ \text { j051 } & 4 & 82 \% & 46 & 33 & 1.521 & 1.583 \\ \text { j052 } & 4 & 85 \% & 48 & 27 & 1.481 & 1.667 \\ \text { j054 } & 4 & 84 \% & 40 & 29 & 1.316 & 1.667 \\ \text { j055 } & 4 & 88 \% & 22 & 22 & 1.424 & 3.000 \\ \text { j056 } & 4 & 94 \% & 39 & 12 & 1.523 & 0.500 \\ \text { j057 } & 4 & 77 \% & 46 & 42 & 1.250 & 2.889\end{array}$


Table 4

Summary of Monti-Carlo Group Information

\begin{tabular}{|c|c|c|c|c|}
\hline Group & Group Size $n=$ & Monti-Carlo & \# of Misses & Subject Group \\
\hline & Monti-Carlo (Human) & Mean Hit Rate & & Med. Hit Rate \\
\hline 1 & $1000(12)$ & $62 \%$ & 70 & $62 \%$ \\
\hline 2 & $1000(11)$ & $77 \%$ & 42 & $77 \%$ \\
\hline 3 & $1000(9)$ & $84 \%$ & 29 & $84 \%$ \\
\hline 4 & $1000(8)$ & $89 \%$ & 21 & $89 \%$ \\
\hline
\end{tabular}


Table 5

Average Number and Length (in Number of Targets) of Hit Runs by Group for Human \& Simulated Subjects

\section{Human Subjects}

Av. \# of Hit Runs Av. Length of Hit

(SD)

Runs (SD)

\section{Simulated Subjects}

Av. \# of Hit Av. Length of Hit

Runs (SD) Runs (SD)

\begin{tabular}{lllll}
\hline Group 1 & $42.667(6.583)$ & $2.839(.657)$ & $43.451(3.141)$ & $2.622(.193)$ \\
Group 2 & $32.455(3.532)$ & $4.384(.686)$ & $32.415(2.458)$ & $4.331(.342)$ \\
Group 3 & $24.889(3.919)$ & $6.423(1.086)$ & $24.437(1.810)$ & $6.201(.517)$ \\
Group 4 & $16.750(5.230)$ & $11.226(4.976)$ & $18.637(1.405)$ & $8.452(.760)$ \\
\hline
\end{tabular}


Table 6

Average number of Hits and Misses following Hits at Short and Long Intervals and Ratios, Short to Long Interval

Hits following Hits $\quad$ Misses following Hits

Short Long Short Long

(Ratio) (Ratio)

\begin{tabular}{lcccc}
\hline Group1 & 43.000 & 31.667 & 26.583 & 16.083 \\
& $(1.36)$ & & $(1.65)$ & \\
Group 2 & 64.182 & 45.636 & 21.909 & 10.545 \\
& $(1.41)$ & & $(2.08)$ & \\
Group 3 & 76.444 & 54.778 & 16.667 & 8.222 \\
& $(1.39)$ & & $(2.04)$ & \\
Group 4 & 88.625 & 60.50 & 11.375 & 5.375 \\
& $(1.46)$ & & $(2.11)$ & \\
\hline
\end{tabular}

Note: Expected ratio short to long was 1.49 


\section{Table 7}

Means and SD of dependent measures for 15 top and 15 bottom performers

\section{Top 15 Bottom 15}

Mean SD Mean SD

\begin{tabular}{lcccc}
\hline Average. Hit Time in ms & 720.204 & 66.055 & 694.658 & 97.131 \\
Number of False Alarms * & 32.800 & 8.529 & 21.933 & 12.595 \\
Ratio Short/Long Hits->Hits & 1.425 & 0.126 & 1.381 & 0.199 \\
Ratio Short/Long Misses->Hits & 2.545 & 1.686 & 1.848 & 0.675 \\
\hline${ }^{*} \mathrm{p}<.01$ & & & & \\
\hline
\end{tabular}


Table 8

Summary of Characteristics for MAW Test Case Functions created in Experiment 2. Part 1

\begin{tabular}{ccc} 
Test Case & $\begin{array}{c}\text { Programmed } \\
\text { Periodicity(ies) }\end{array}$ & $\begin{array}{c}\text { Programmed Noise } \\
\text { Level }\end{array}$ \\
\hline 1 & 2 Minutes & None \\
3 & 2 Minutes & Low \\
4 & 2 Minutes & Moderate \\
5 & 2 Minutes & High \\
6 & $2 \& 4$ Minutes & None \\
7 & $1 \& 4$ Minutes & None \\
& $1 \& 5$ Minutes & None
\end{tabular}


Table 9

Primary Frequencies in Minutes per Cycle from the Accuracy FFT. Subjects with Best Power Only

4 Primary Peaks Sorted by Power Subject Peak 1 Peak 2 Peak 3 Peak 4

$\begin{array}{ccccc}\text { Group 1 } & 9 & 5.1 & 1.1 & \\ 10 & 5.4 & 3.1 & & \\ 12 & 5.7 & 2 & 1.5 & \\ 13 & 4.9 & 12.8 & 1.1 & 1.6 \\ 16 & 2 & 1.5 & 1.2 & \\ 35 & 4.7 & 1.7 & 1.4 \\ 34 & 4.9 & 17 & 1.7 \\ 25 & 7.9 & 1.7 & \end{array}$

Group 2

22

4.7

3.1

2

50

11.4

2

5.1

48

1.8

5.1

25.6

Group 3

$31 \quad 1.8$

37

5.4

1.9

1.3

39

1.9

5.4

1.3

49

5.4

1.9

Group 4

36

3.4

1.5 
Table 10

Mean and SD in Minutes per Cycle and Percentage of Subjects with a

Frequency in Each Bin for Human Subjects based on Original Bins

Human Subjects $(\underline{n}=40)$

\begin{tabular}{lrc}
\hline Bin 1 & Mean & 1.213 \\
(0-1.5 Minutes per Cycle) & SD & .184 \\
& $\%$ & 70 \\
Bin 2 & Mean & 2.227 \\
(1.51-3.5 Minutes per Cycle) & SD & .546 \\
\cline { 2 - 3 } & $\%$ & 85 \\
Bin 3 & Mean & 5.149 \\
(3.51-8.5 Minutes per Cycle) & SD & 1.079 \\
\cline { 2 - 3 } Bin 4 & $\%$ & 73 \\
(8.51- Minutes per Cycle) & Mean & 13.898 \\
\cline { 2 - 3 } & SD & 3.130 \\
\hline
\end{tabular}


Table 11

Summary of Narrowed Bin ranges, and actual ranges for Human and Simulated Subjects in Minutes

\begin{tabular}{ll}
\hline Narrowed Ranges & Actual Ranges \\
in Min. per Cycle &
\end{tabular}

\begin{tabular}{lccc} 
& & Human & Simulated \\
\hline Bin 1 & $.94-1.49$ & $.993-1.461$ & $.984-1.461$ \\
Bin 2 & $1.41-3.05$ & $1.550-2.923$ & $1.527-3.009$ \\
Bin 3 & $3.53-6.77$ & $3.654-6.394$ & $3.528-6.018$ \\
Bin 4 & $9.2-18.59$ & $9.3-17.5$ & $9.3-14.614$ \\
\hline
\end{tabular}


Table 12

Percentage of Human and Modeled Subjects as members of narrowed Bins Human Subjects $(\underline{n}=40) \quad$ Simulated Subjects $(\underline{n}=40)$

\begin{tabular}{lll}
\hline Bin 1 & 60 & 53 \\
Bin 2 & 75 & 58 \\
Bin 3 & 63 & 50 \\
Bin 4 & 30 & 13 \\
\hline
\end{tabular}


Table 13

Percentage Membership for human subject groupings by FFT periodicity range bin.

Bin \& Frequency Range

1

23

4

$$
\text { 0.94-1.49 } \quad 1.41-3.05 \quad 3.53-6.77 \quad 9.20-18.59
$$

\begin{tabular}{lllll}
\hline Group 1 $(\mathrm{n}=12)$ & $75 \%$ & $83 \%$ & $67 \%$ & $33 \%$ \\
Group 2 $(\mathrm{n}=11)$ & $55 \%$ & $73 \%$ & $64 \%$ & $36 \%$ \\
Group 3 $(\mathrm{n}=9)$ & $67 \%$ & $89 \%$ & $56 \%$ & $11 \%$ \\
Group 4 $(\mathrm{N}=8)$ & $38 \%$ & $50 \%$ & $63 \%$ & $38 \%$ \\
\hline
\end{tabular}


Table 14

Primary Frequencies in Minutes per Cycle from the Reaction Time FFT. Subjects with Best Power Only

4 Primary Peaks Sorted by Power Subject Peak 1 Peak 2 Peak 3 Peak 4

\begin{tabular}{|c|c|c|c|c|c|}
\hline Group 1 & 9 & 4.4 & 1.1 & 0.9 & \\
\hline & 10 & 5.7 & 3.2 & 1.3 & \\
\hline & 12 & 2.8 & 6 & 1.2 & \\
\hline & 13 & 5.4 & & & \\
\hline & 16 & 2 & 1.5 & & \\
\hline & 34 & 14.6 & 4.7 & & \\
\hline & 25 & 1.7 & 7.3 & 3.6 & \\
\hline Group 2 & 22 & 3.1 & 4.7 & 2 & 1.3 \\
\hline & 57 & 1.9 & 14.6 & & \\
\hline & 50 & 10.2 & 4.9 & & \\
\hline & 48 & 1.8 & 25.6 & 4.9 & \\
\hline & 43 & 1.3 & 0.9 & 1.7 & 2.2 \\
\hline & 32 & 20.5 & 1.1 & 6 & 1.9 \\
\hline & 30 & 14.6 & 4.1 & & \\
\hline
\end{tabular}

Group 3

37

5.1 


$\begin{array}{lll}39 & 5.7 & 1.9 \\ 45 & 8.5 & 1.4 \\ 49 & 4.9 & 1.9 \\ 52 & 6 & 1.3\end{array}$

Group $4 \quad 55 \quad 11.4$

$\begin{array}{lll}36 & 3.2 & 1.2\end{array}$


Table 15

Summary of Narrowed Bin ranges in Minutes per Cycle, and actual ranges for Human Subject (Accuracy \& Reaction Time)

Narrowed Ranges $\quad$ Actual Ranges

$\begin{array}{llll}\text { Accuracy } & \text { RT } & \text { Accuracy } & \text { RT }\end{array}$

\begin{tabular}{lcccc}
\hline Bin 1 & $.94-1.49$ & $.99-1.39$ & $.993-1.461$ & $.993-1.382$ \\
Bin 2 & $1.41-3.05$ & $1.5-3.21$ & $1.550-2.923$ & $1.598-3.197$ \\
Bin 3 & $3.53-6.77$ & $3.61-7.05$ & $3.654-6.394$ & $3.935-6.620$ \\
Bin 4 & $9.2-18.59$ & $1.91-37.69$ & $9.3-17.5$ & $8.525-34.1$ \\
\hline
\end{tabular}


Table 16

Summary of Reaction Time and Accuracy FFT results in Minutes per Cycle and Percent of Subjects with a Peak in each Bin for Human Subjects based on Narrowed Bins

$$
\text { RT Results }(n=40) \quad \text { Accuracy Results }(n=40)
$$

\begin{tabular}{lrrrr}
\hline Bin 1 & Mean & 1.172 & Mean & 1.242 \\
& SD & .119 & SD & .146 \\
& $\%$ & 66 & $\%$ & 60 \\
Bin 2 & Mean & 2.284 & Mean & 2.053 \\
& SD & .522 & SD & .425 \\
& $\%$ & 66 & $\%$ & 75 \\
Bin 3 & Mean & 5.276 & Mean & 4.952 \\
& SD & .873 & SD & .767 \\
& $\%$ & 63 & $\%$ & 63 \\
Bin 4 & Mean & 17.186 & Mean & 13.898 \\
& SD & 7.776 & SD & 3.130 \\
& $\%$ & 58 & $\%$ & 30 \\
\hline
\end{tabular}


Table 17

Percentage of Human Subjects Categorized for Clarity of Primary Frequency Power for Accuracy and RT

Primary Frequency Power

$\begin{array}{ll}\text { Accuracy } & \text { RT }\end{array}$

Clear

$65.0 \%$

$65.0 \%$

Moderately Clear

$32.5 \%$

$27.5 \%$

Not Clear

$2.5 \%$

$7.5 \%$ 
Table 18

Overall Noise Level in Accuracy and RT Spectra for Human Subjects

Overall Noise Level

Accuracy RT

\begin{tabular}{lcc}
\hline High & $10.0 \%$ & $5.0 \%$ \\
Medium & $37.5 \%$ & $22.5 \%$ \\
Low & $52.5 \%$ & $72.5 \%$ \\
\hline
\end{tabular}


Table 19

Summary of the Comparability of Primary Frequency Peaks for Accuracy and Reaction Time Spectra

Comparability of Primary Frequencies

Accuracy vs. RT

Same

Similar

Different
$42.5 \%$

$40.0 \%$

$17.5 \%$ 
Table 20

Percentage Membership for Human Subject Groupings by RT FFT Periodicity Range Bin.

Bin \& Frequency Range (in Minutes per Cycle)

1

2

3

4

$$
\text { 0.99-1.39 } \quad 1.50-3.21 \quad 3.61-7.05 \quad 1.91-37.69
$$

\begin{tabular}{lllll}
\hline Group 1 $(\mathrm{n}=12)$ & $67 \%$ & $75 \%$ & $75 \%$ & $33 \%$ \\
Group 2 $(\mathrm{n}=11)$ & $64 \%$ & $82 \%$ & $64 \%$ & $73 \%$ \\
Group 3 $(\mathrm{n}=9)$ & $67 \%$ & $44 \%$ & $56 \%$ & $78 \%$ \\
Group 4 $(\mathrm{N}=8)$ & $75 \%$ & $63 \%$ & $50 \%$ & $50 \%$ \\
\hline
\end{tabular}




\section{Histogram: Number of Targets Appearing in Windows}

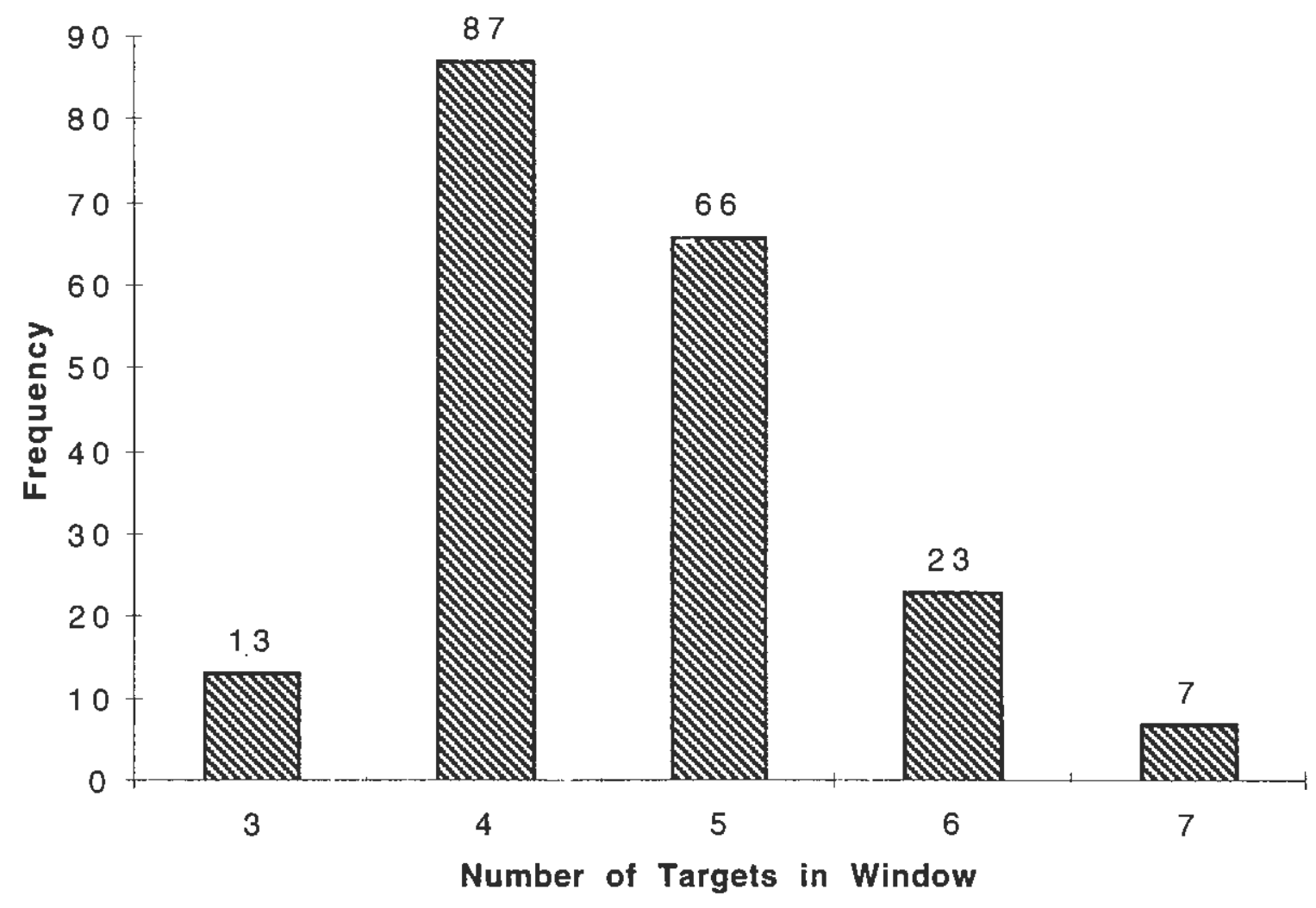

Figure 1. The distribution of window sizes (number of targets falling within the 30 second window) for the MAW function on the CPT template. 


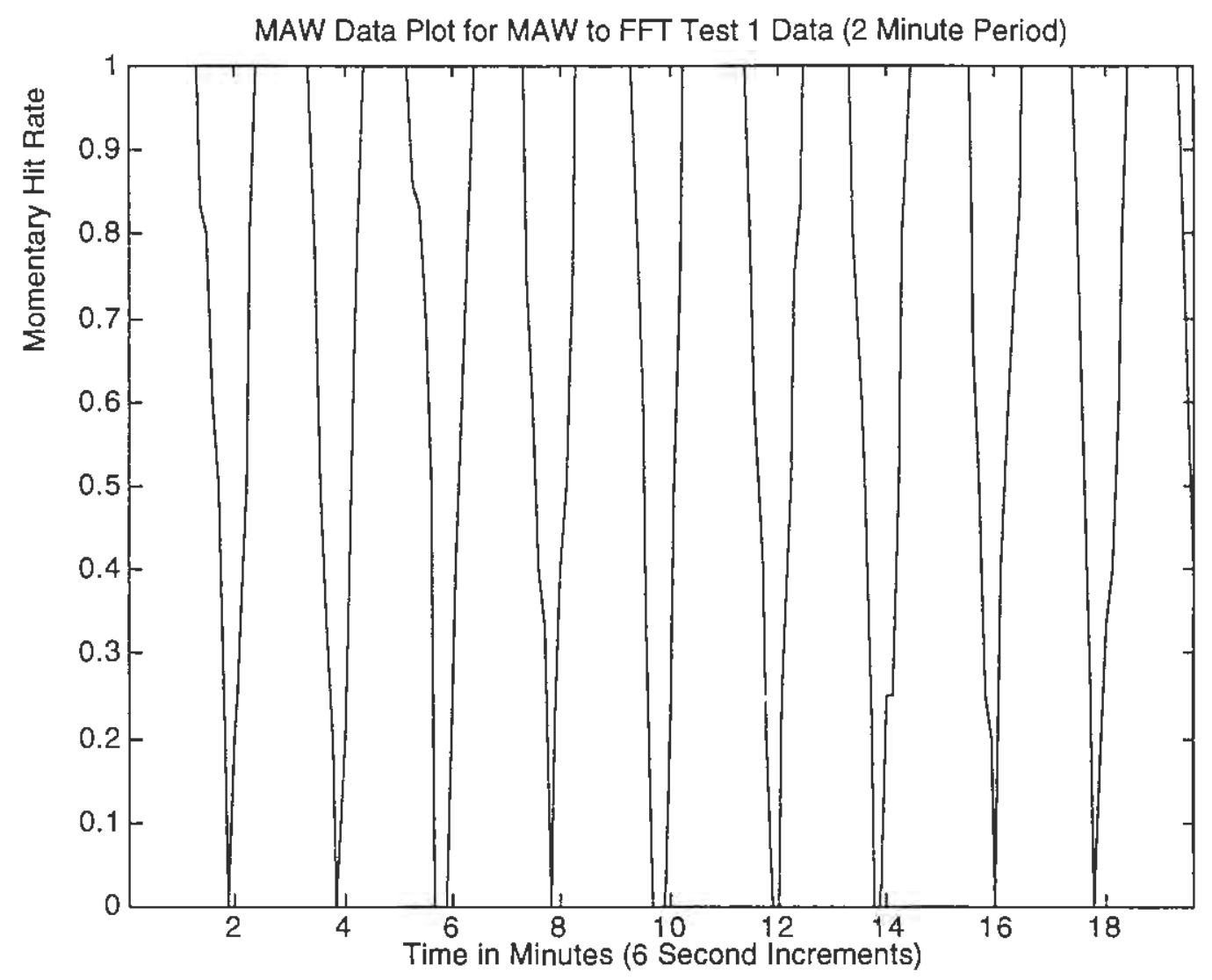

Figure 2. MAW Data Plot for Case 1. Hits (correct target detections) were arranged to occur with 2 minute periodicity (No noise) 


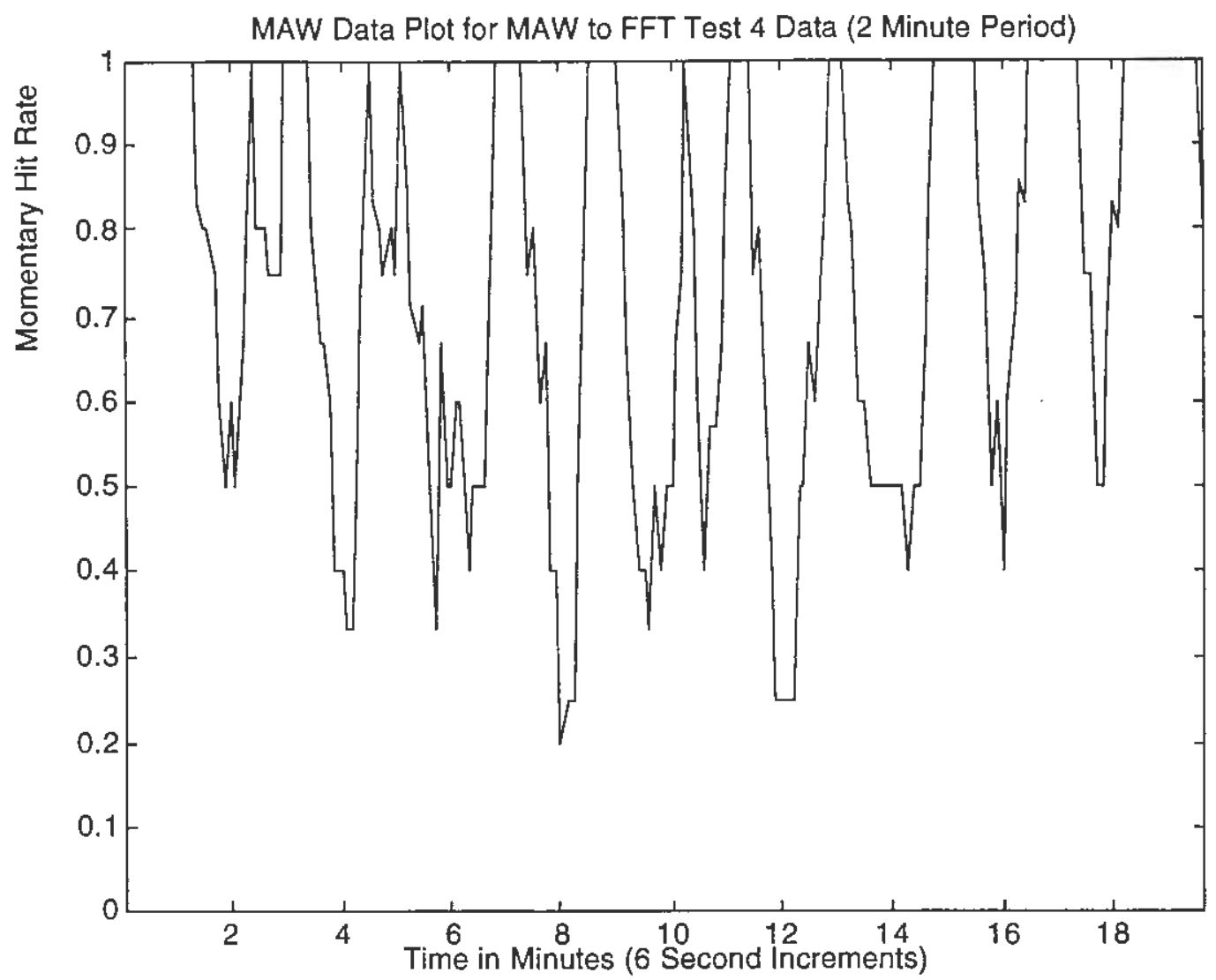

Figure 3. MAW Data Plot for Case 4. Hits were arrange to occur with a 2 Minute Periodicity (High Noise.) 


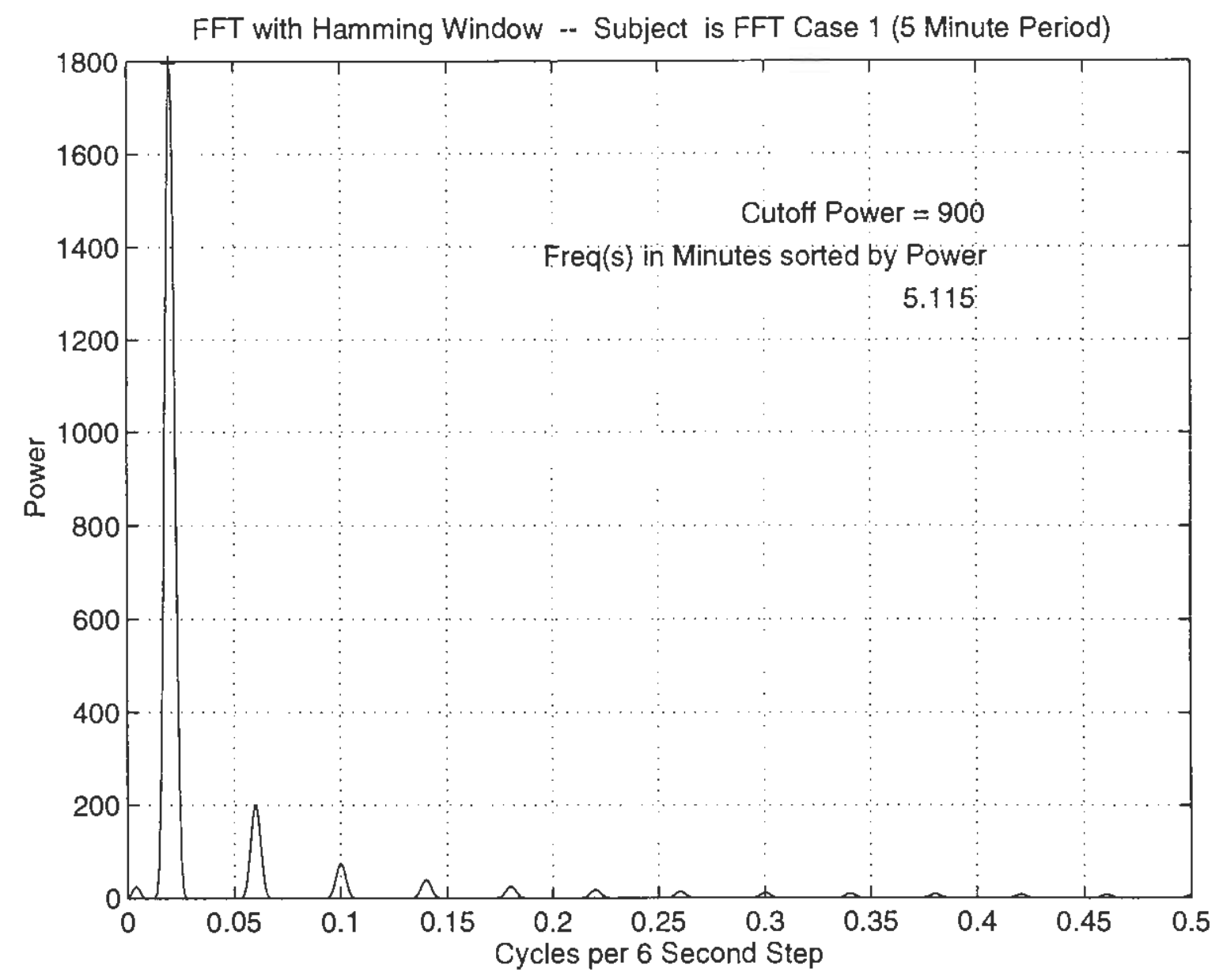

Figure 4. FFT power spectrum for Case 1. Hit runs were arrange to occur with a 5 Minute Periodicity. Calulations of frequency periods in minutes are shown. 


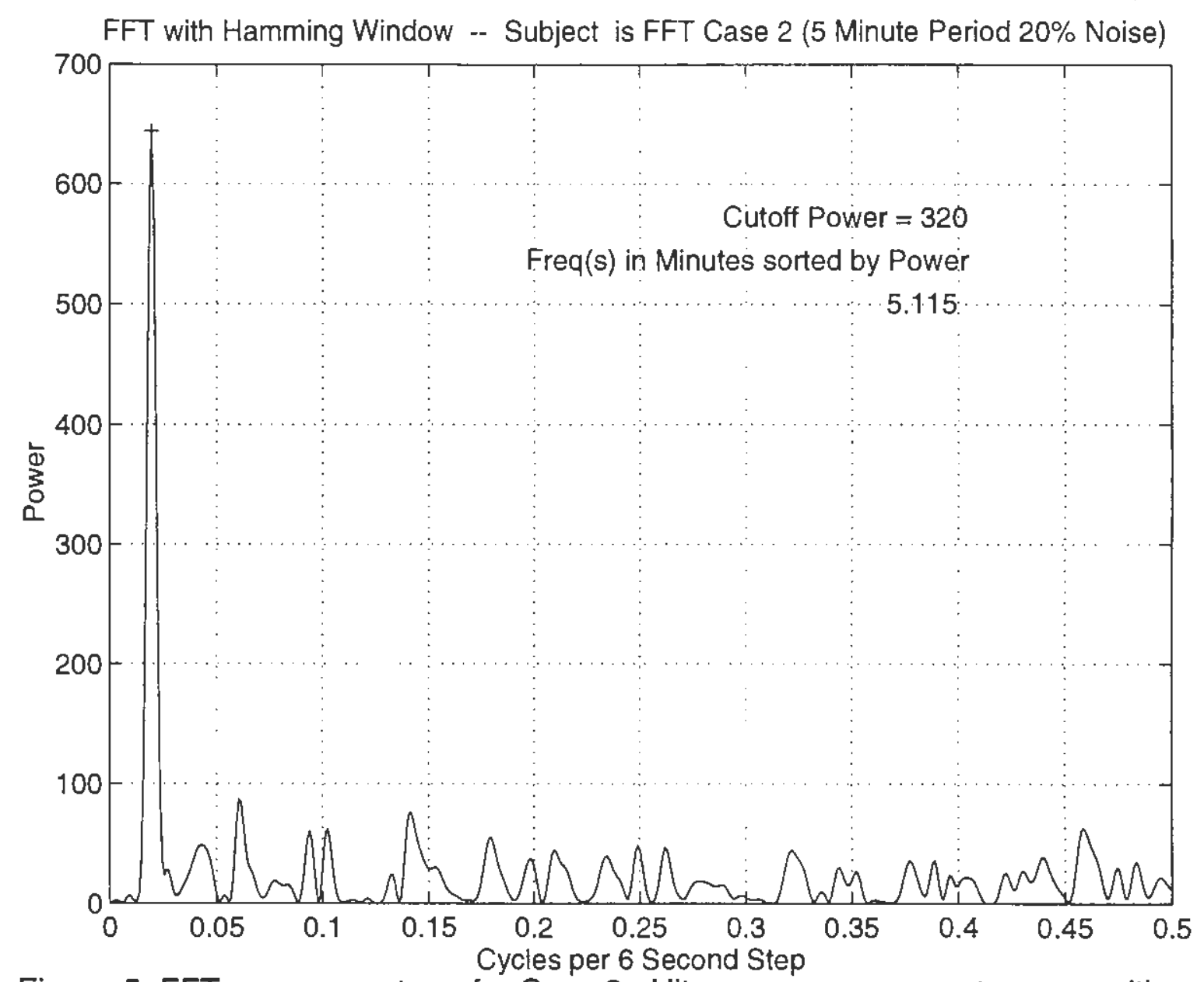

Figure 5. FFT power spectrum for Case 2. Hit runs were arrange to occur with a 5 Minute Periodicity with $20 \%$ noise. Calulations of frequency periods in minutes are shown. 


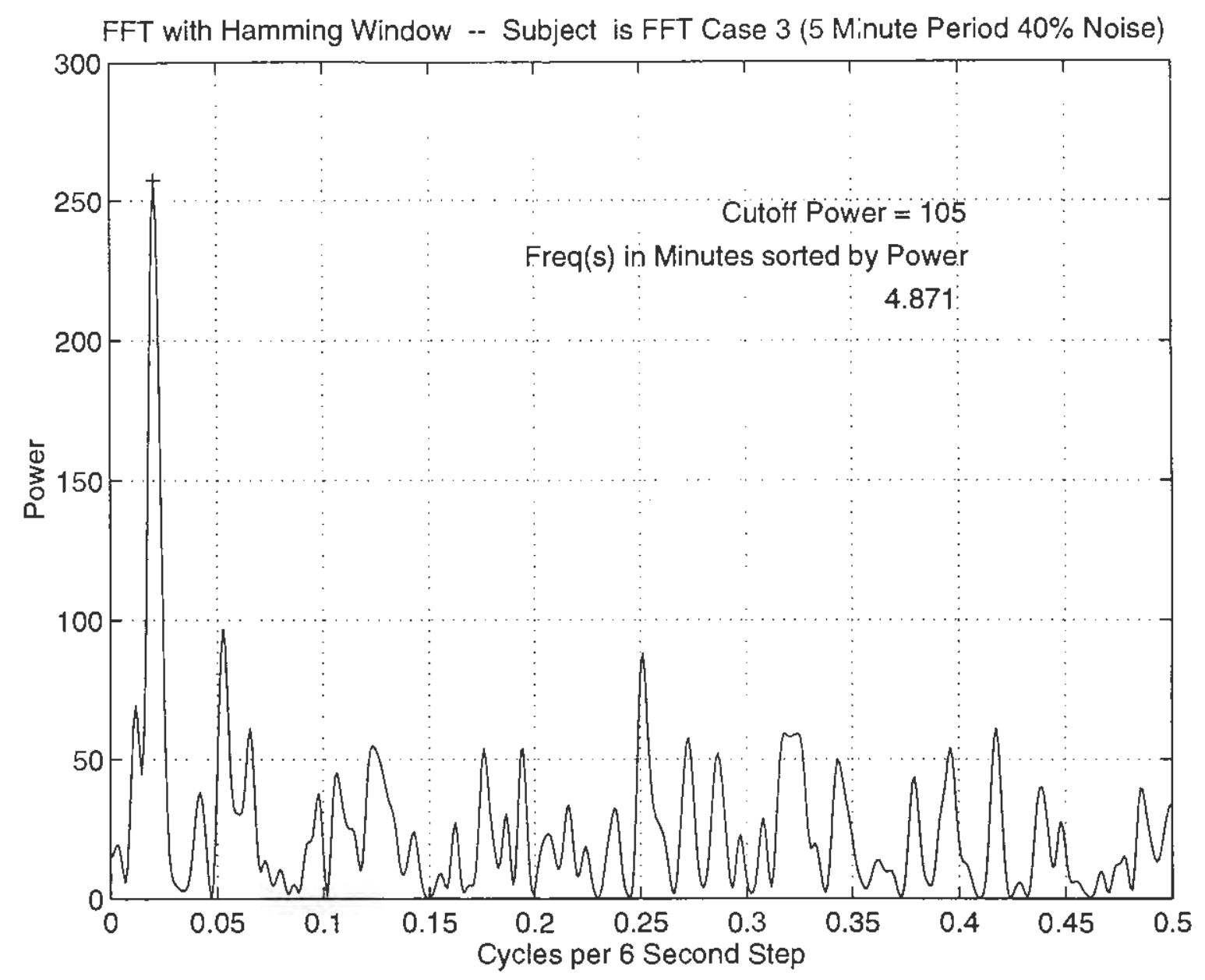

Figure 6. FFT power spectrum for Case 3. Hit runs were arrange to occur with a 5 Minute Periodicity with $40 \%$ noise. Calulations of frequency periods in minutes are shown. 


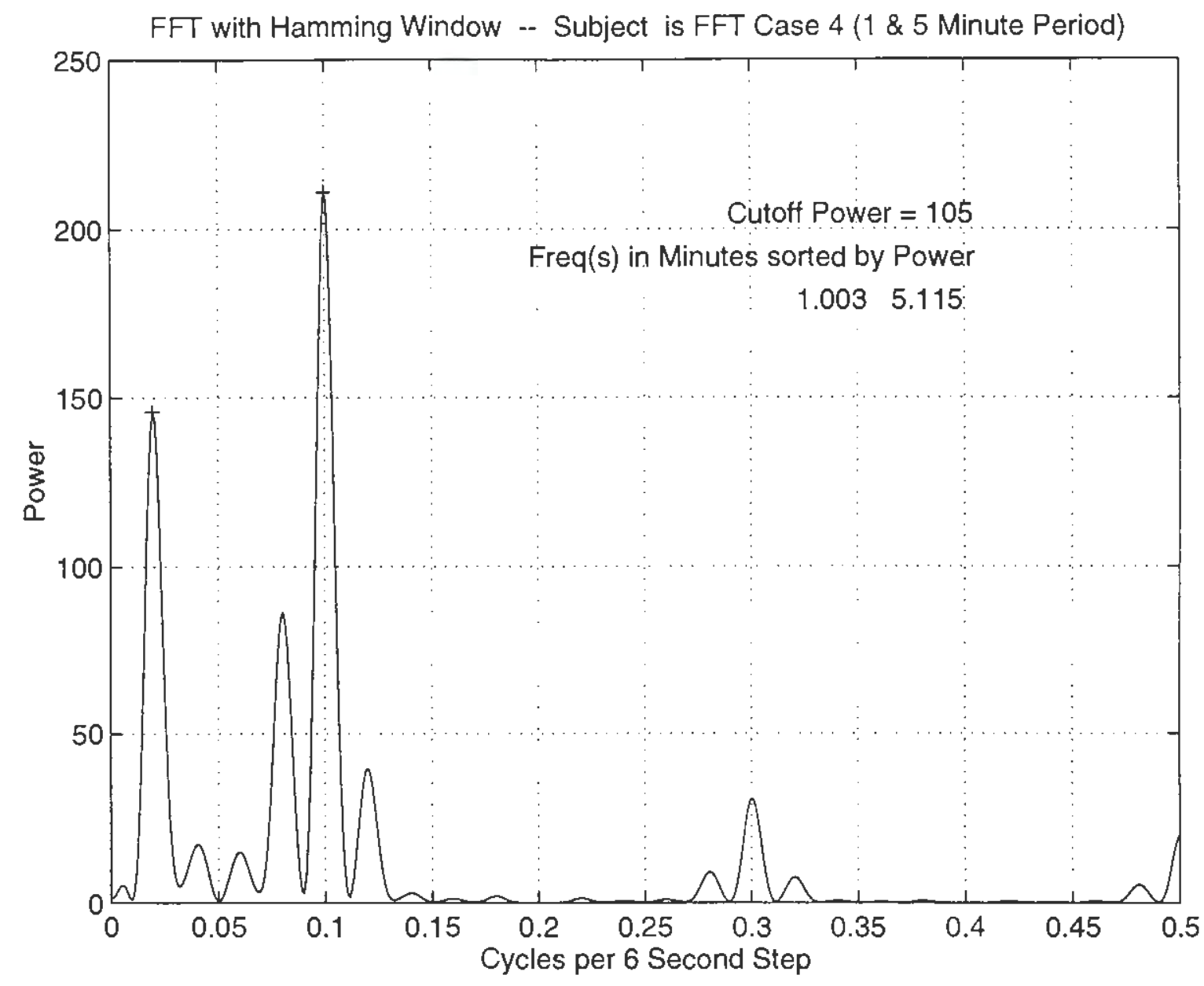

Figure 7. FFT power spectrum for Case 4. Hit runs were arrange to occur with 1 and 5 Minute Periodicities. Calulations of frequency periods in minutes are shown. 


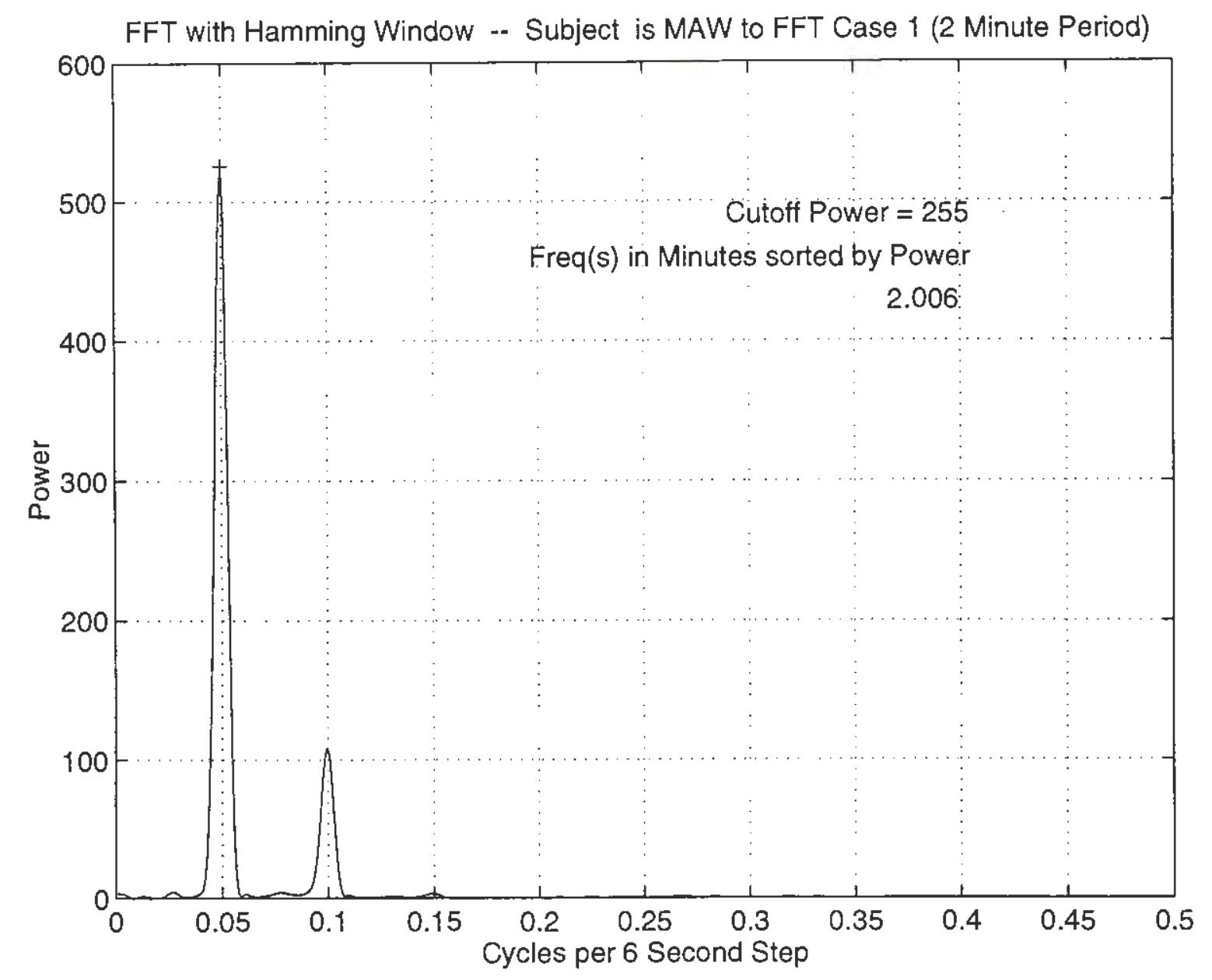

Figure 8. MAW data FFT power spectrum for Case 1. Hit runs were arrange to occur with 2 Minute Periodicity. Calulations of frequency periods in minutes are shown. 
FFT with Hamming Window -- Subject is MAW to FFT Case 2 (2 Minute Period Low Noise)

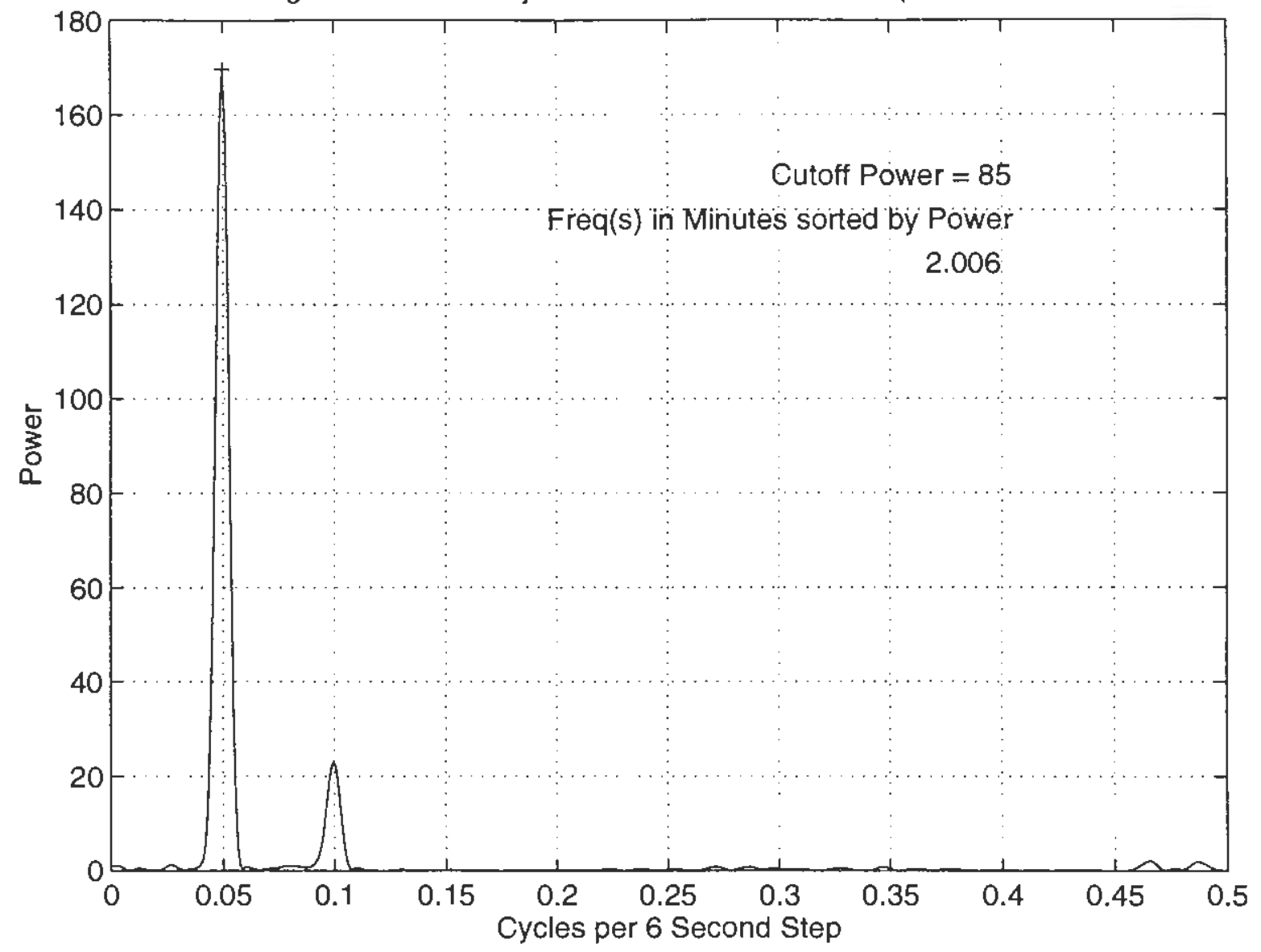

Figure 9. MAW data FFT power spectrum for Case 2. Hit runs were arrange to occur with 2 Minute Periodicity and a low level of noise was added. Calulations of frequency periods in minutes are shown. 
FFT with Hamming Window -- Subject is MAW to FFT Case 3 (2 Minute Period Moderate Noise)

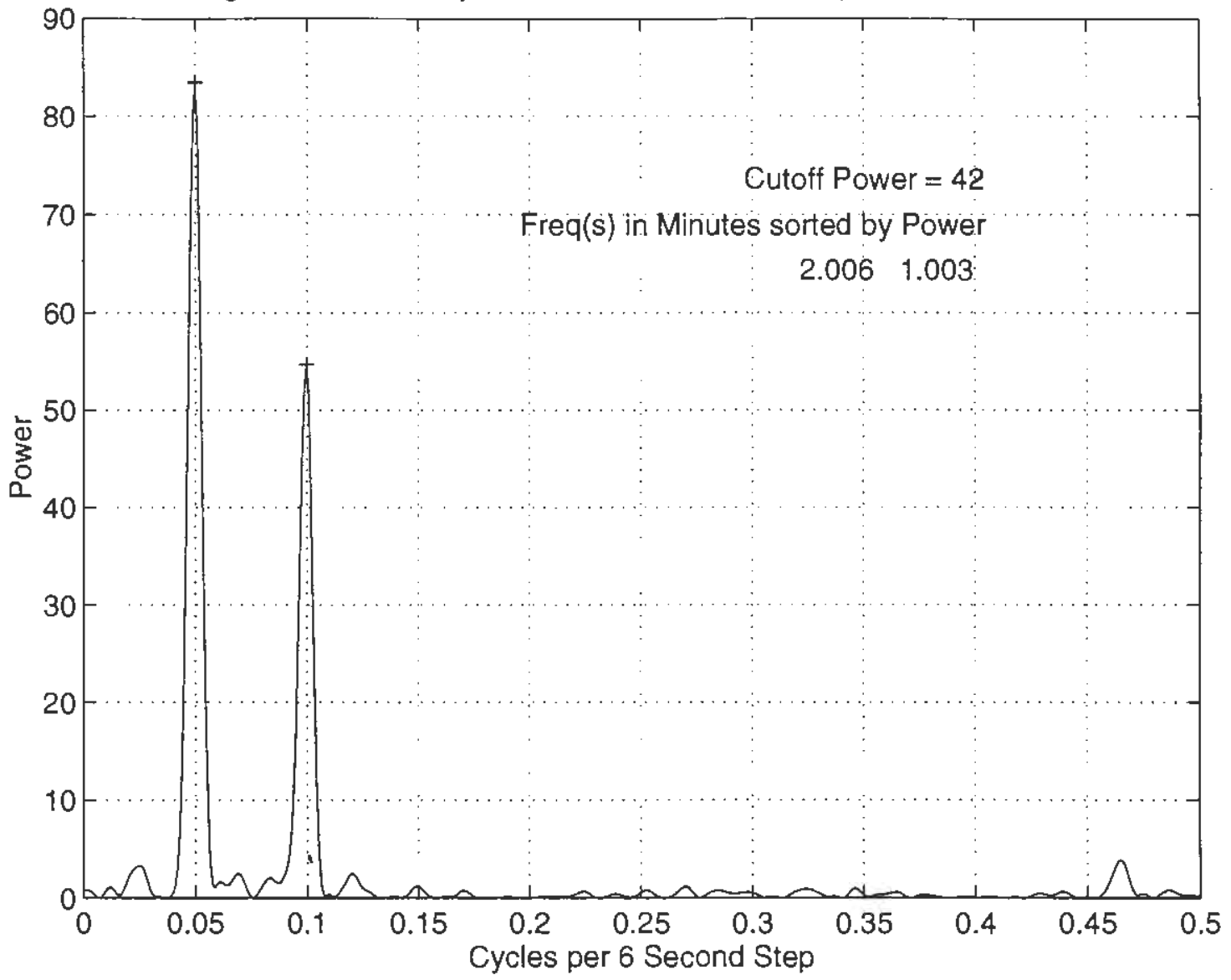

Figure 10. MAW data FFT power spectrum for Case 3. Hit runs were arrange to occur with 2 Minute Periodicity and a moderate level of noise was added. Calulations of frequency periods in minutes are shown. 
FFT with Hamming Window -- Subject is MAW to FFT Case 4 (2 Minute Period High Noise)

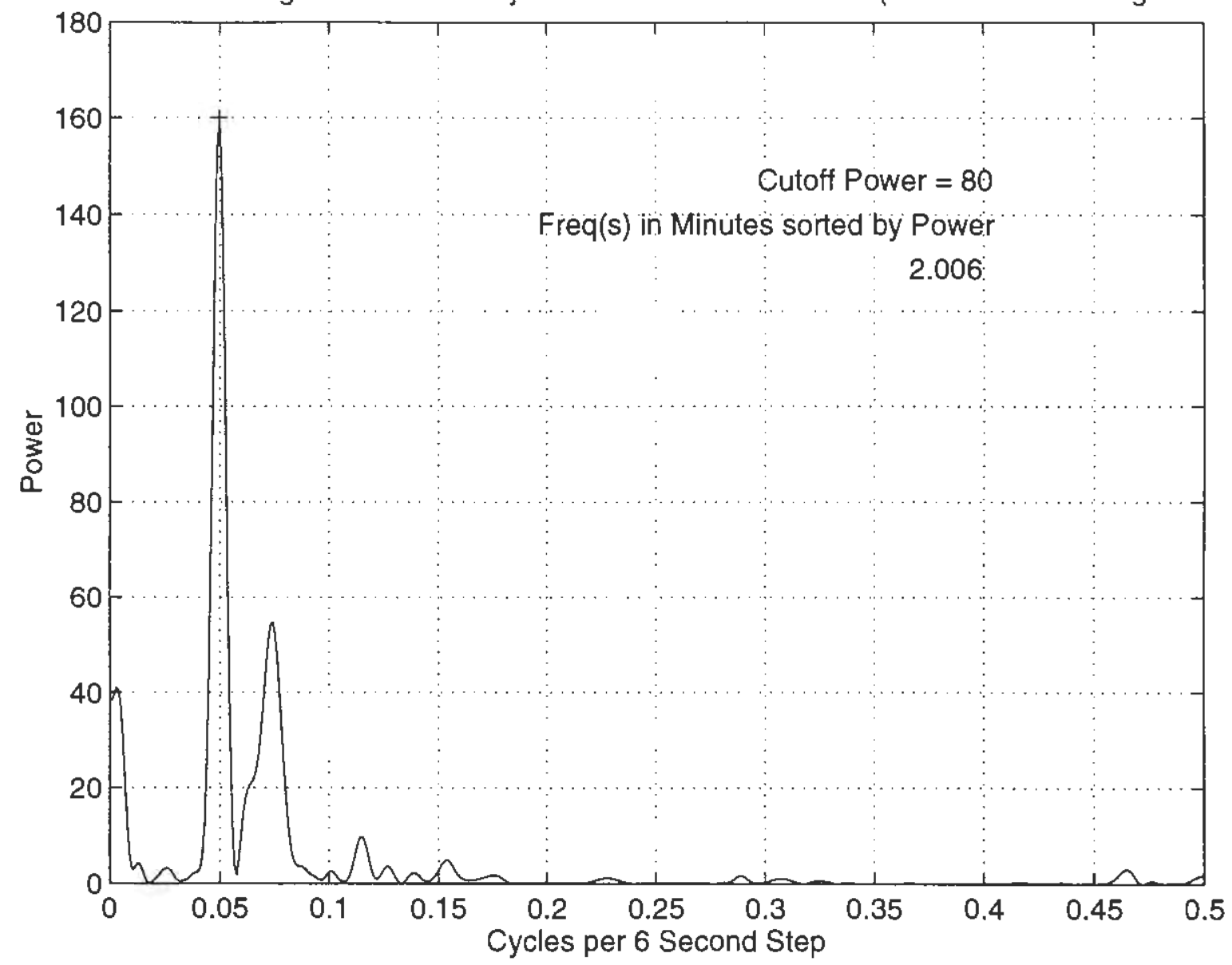

Figure 11. MAW data FFT power spectrum for Case 4. Hit runs were arrange to occur with 2 Minute Periodicity and a high level of noise was added.

Calulations of frequency periods in minutes are shown. 


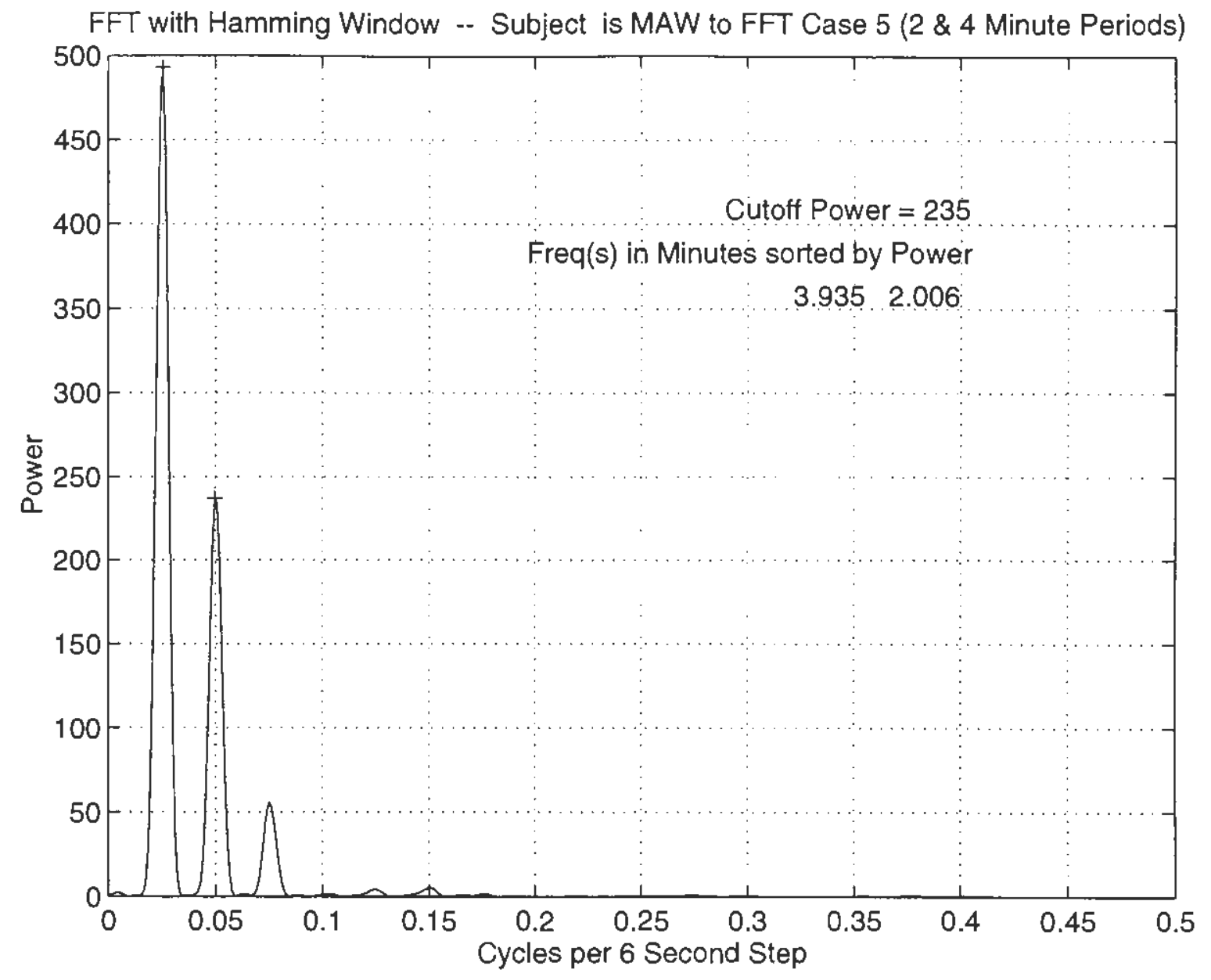

Figure 12. MAW data FFT power spectrum for Case 5 . Hit runs were arrange to occur with 2 and 4 Minute Periodicities. Calulations of frequency periods in minutes are shown. 


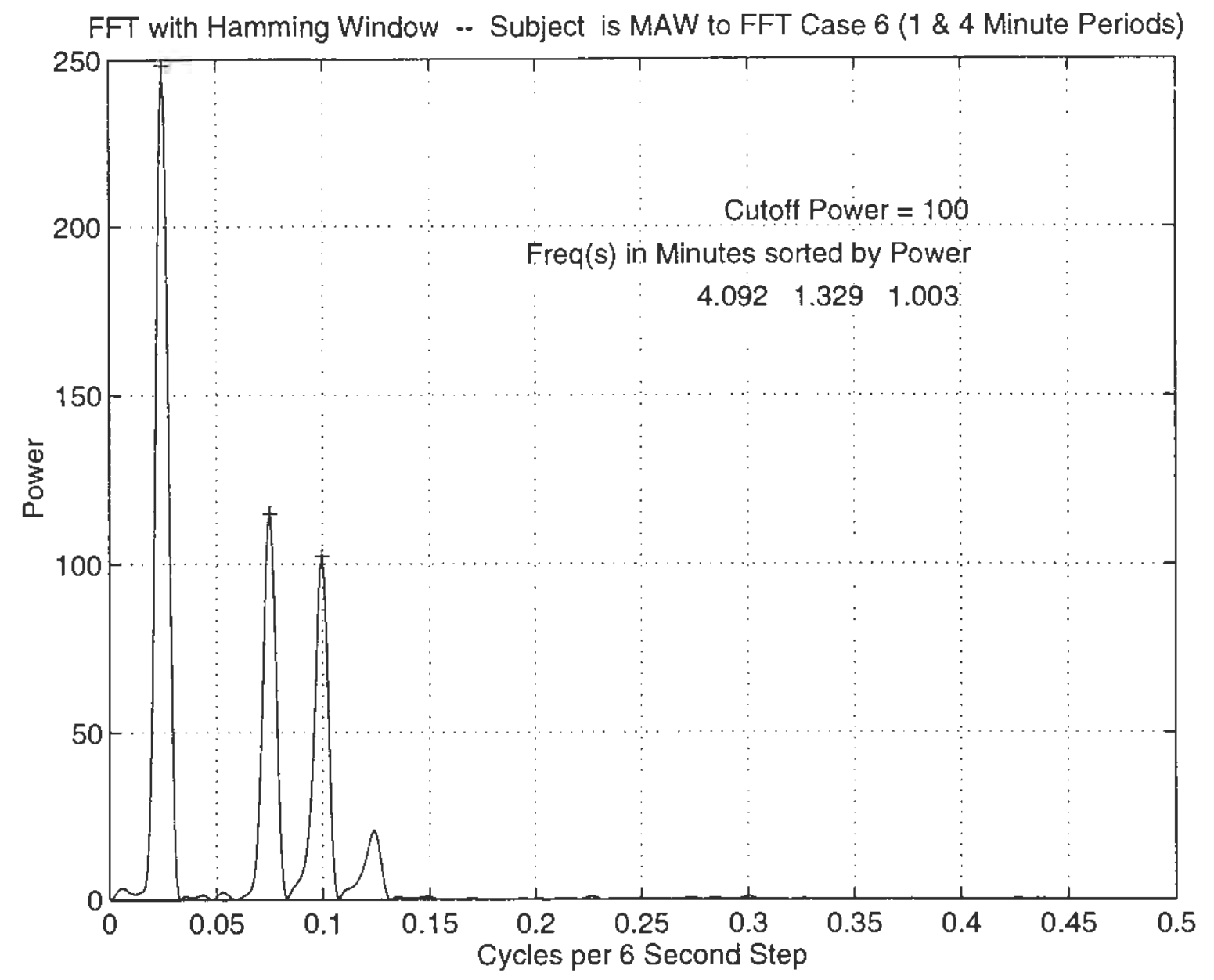

Figure 13. MAW data FFT power spectrum for Case 6 . Hit runs were arrange to occur with 1 and 4 Minute Periodicities. Calulations of frequency periods in minutes are shown. 
FFT with Hamming Window .. Subject is MAW to FFT Case 7 ( 1 \& 5 Minute Periods)

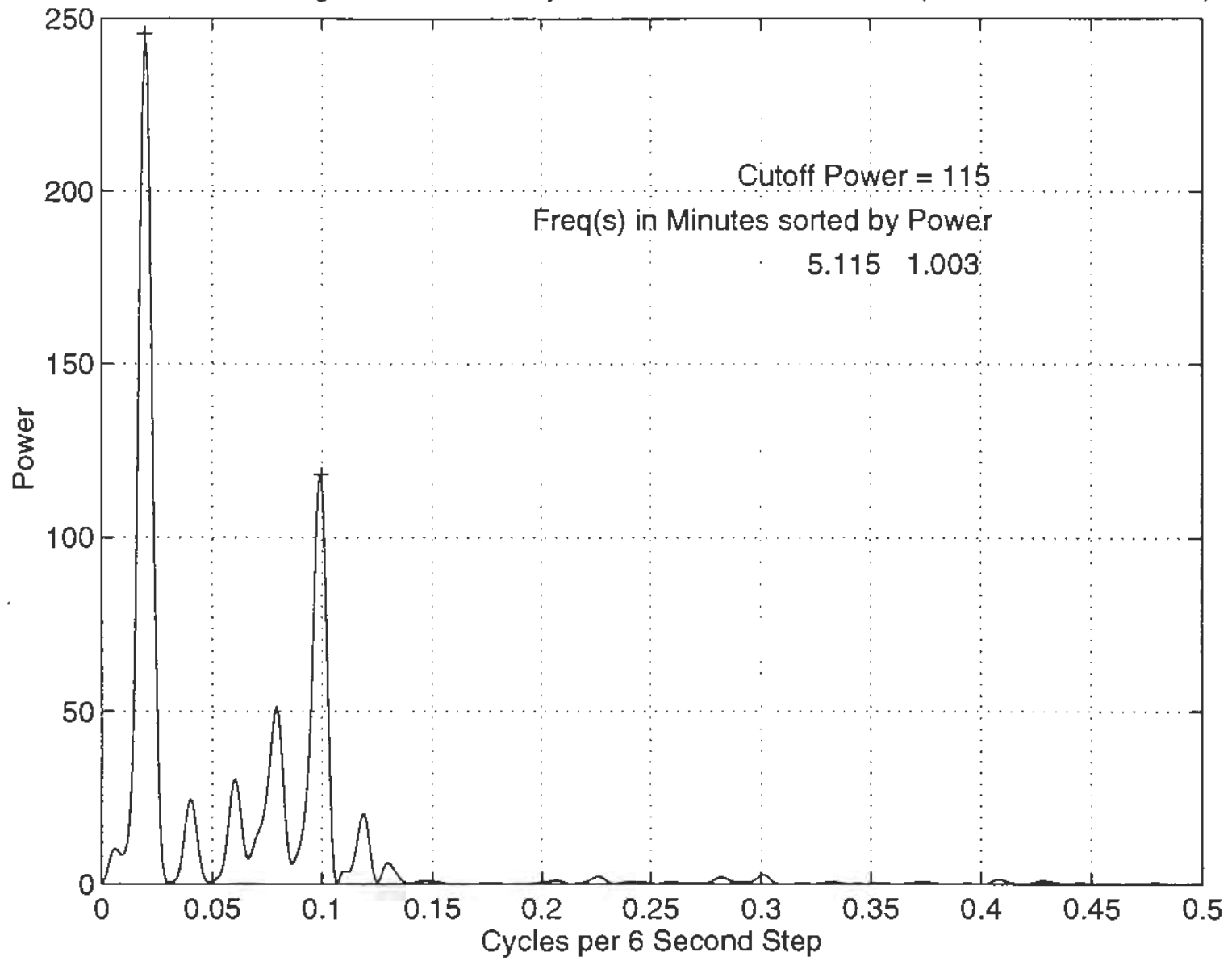

Figure 14. MAW data FFT power spectrum for Case 7. Hit runs were arrange to occur with 1 and 5 Minute Periodicities. Calulations of frequency periods in minutes are shown. 


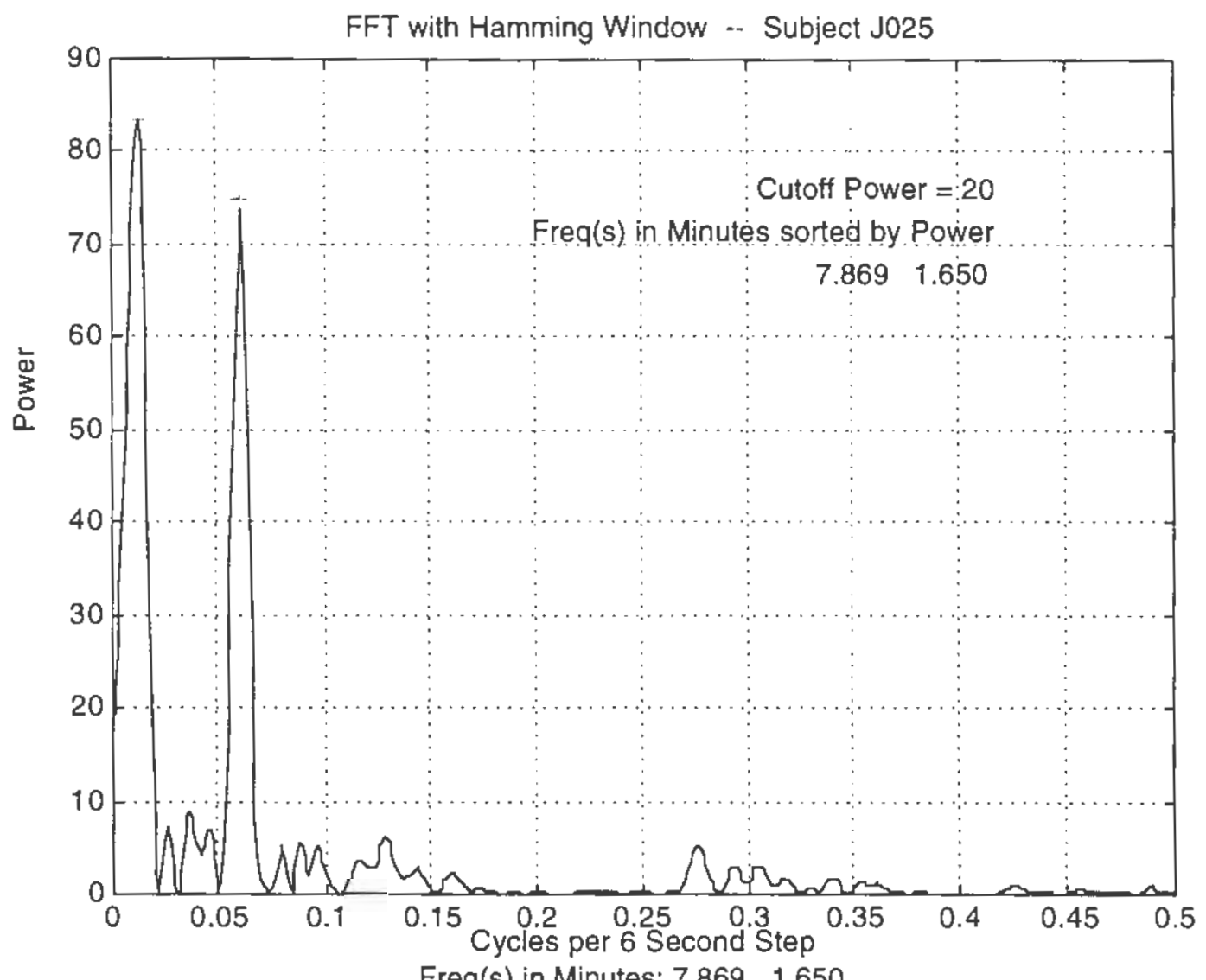

Freq(s) in Minutes: $7.869 \quad 1.650$

Figure 15. FFT Power Spectrum showing a good example of high primary frequency clarity. 


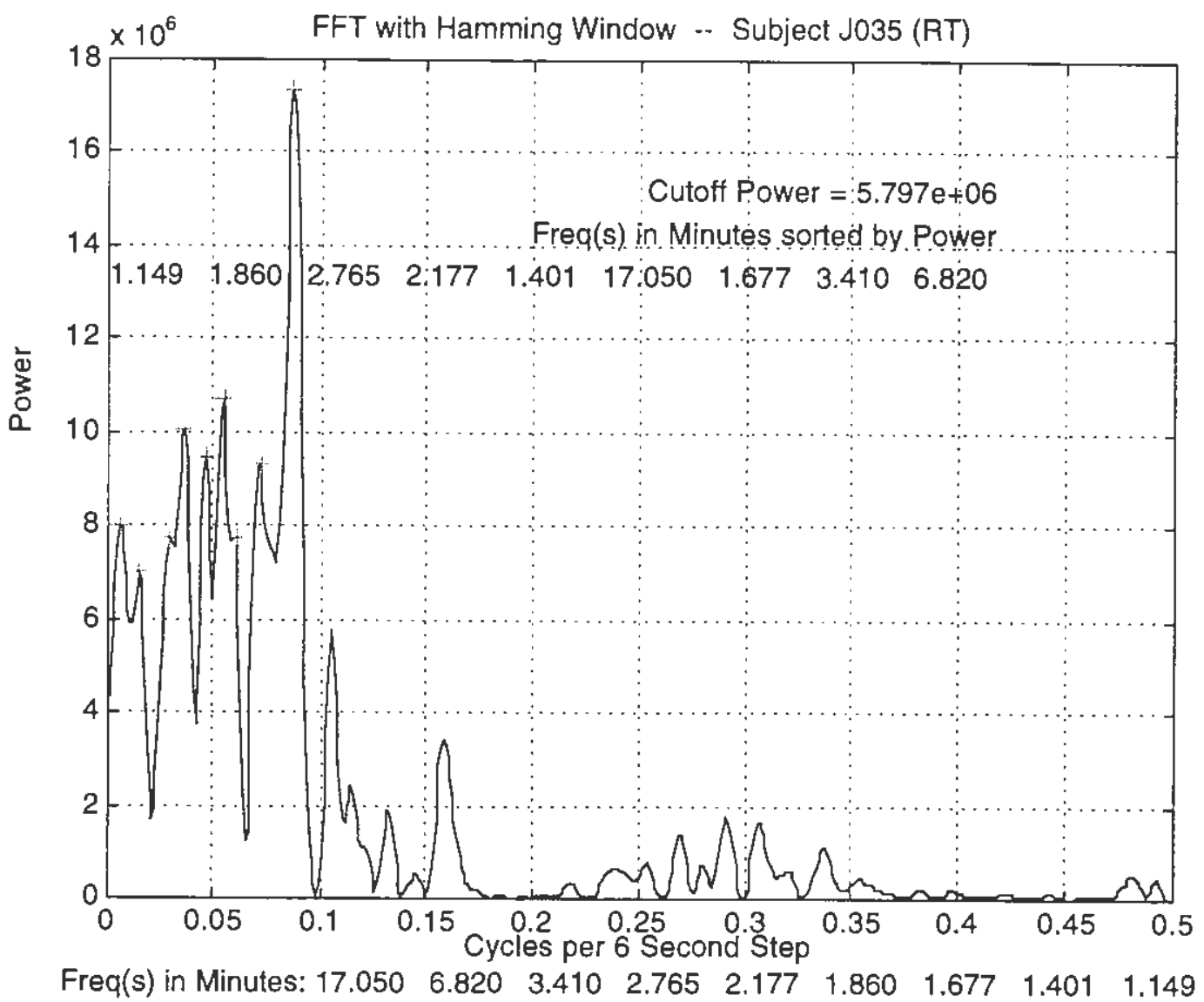

Figure 16. FFT Power Spectrum showing a good example for low primary frequency clarity. 


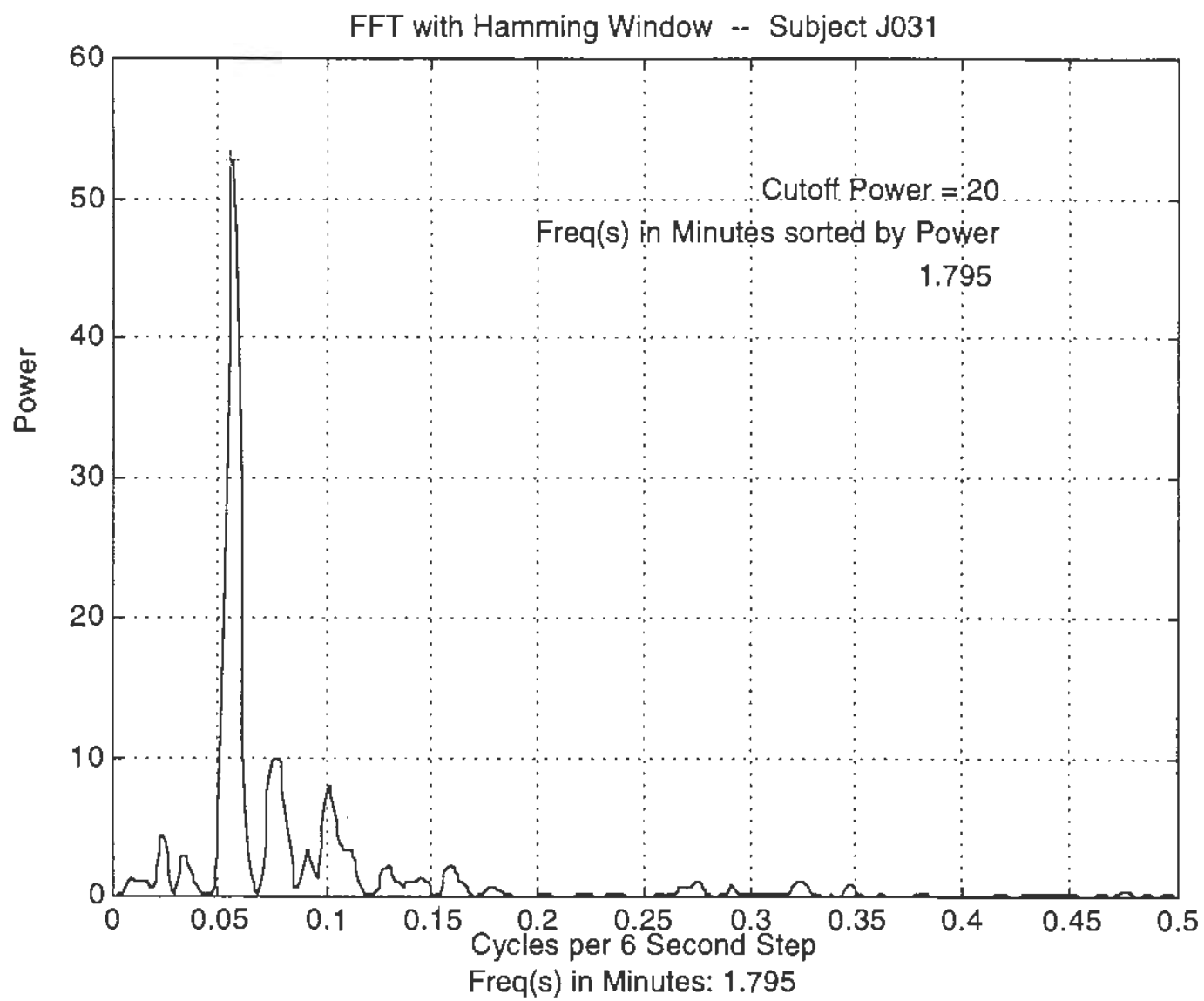

Figure 17. FFT Power Spectrum showing a good example of an overall Low noise level on a power spectrum. 


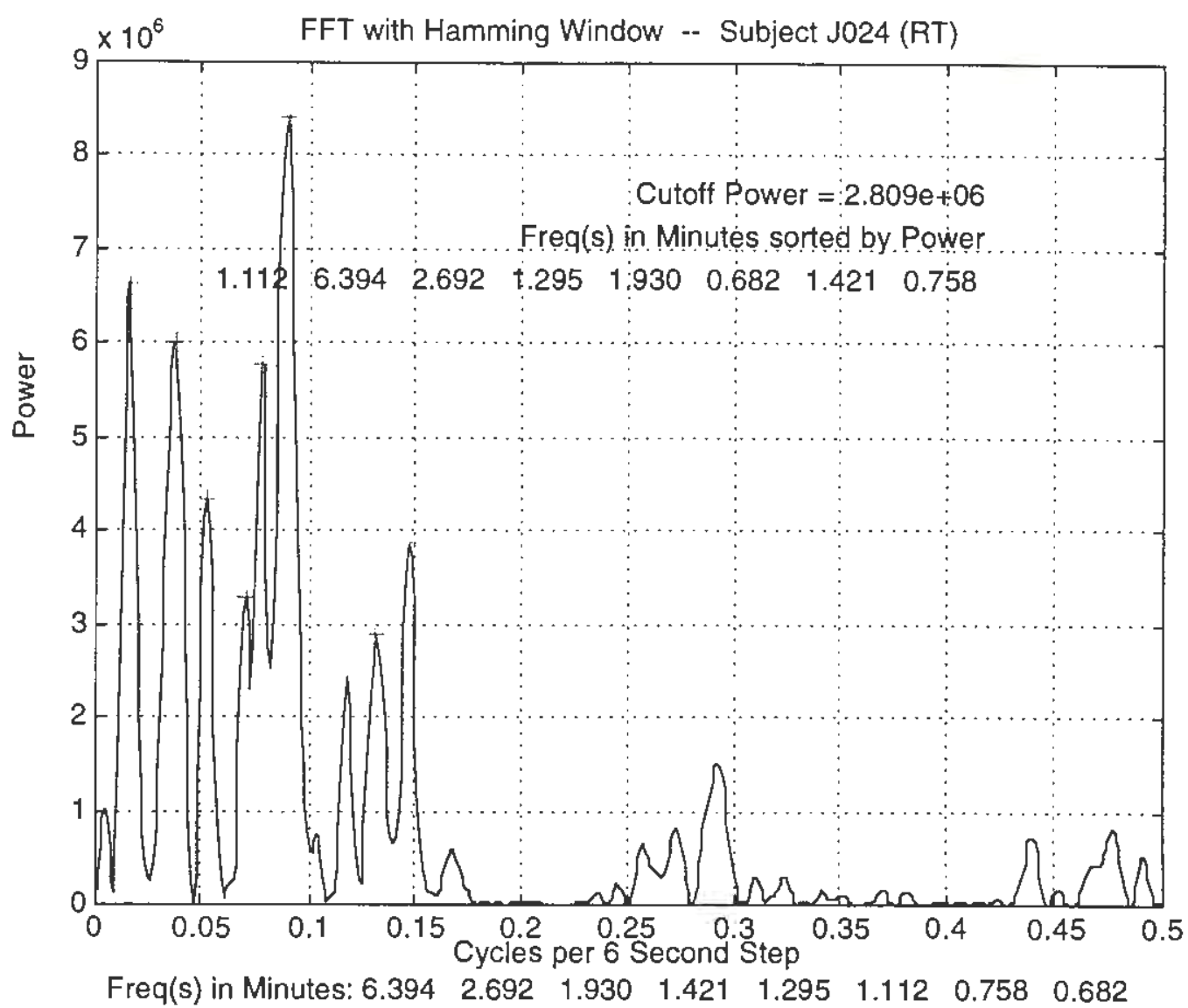

Figure 18. FFT Power Spectrum showing a good example of a high overall noise level in a power spectrum. 

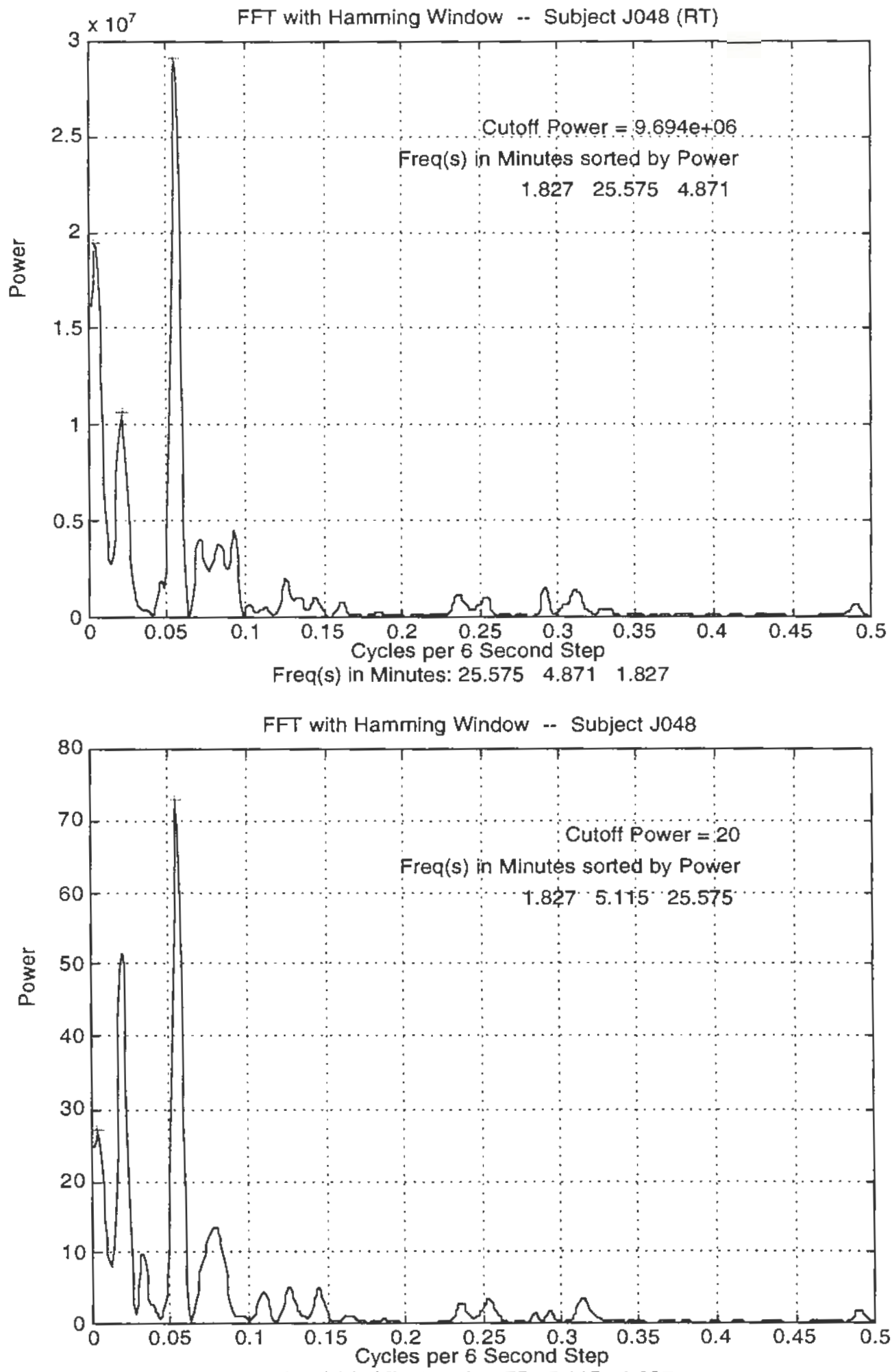

Freq(s) in Minutes: $25.575 \quad 5.115 \quad 1.827$

Figure 19. Power Spectra (RT and Accuracy) for Human Subject 48, rated as Same for primary frequency bands. 

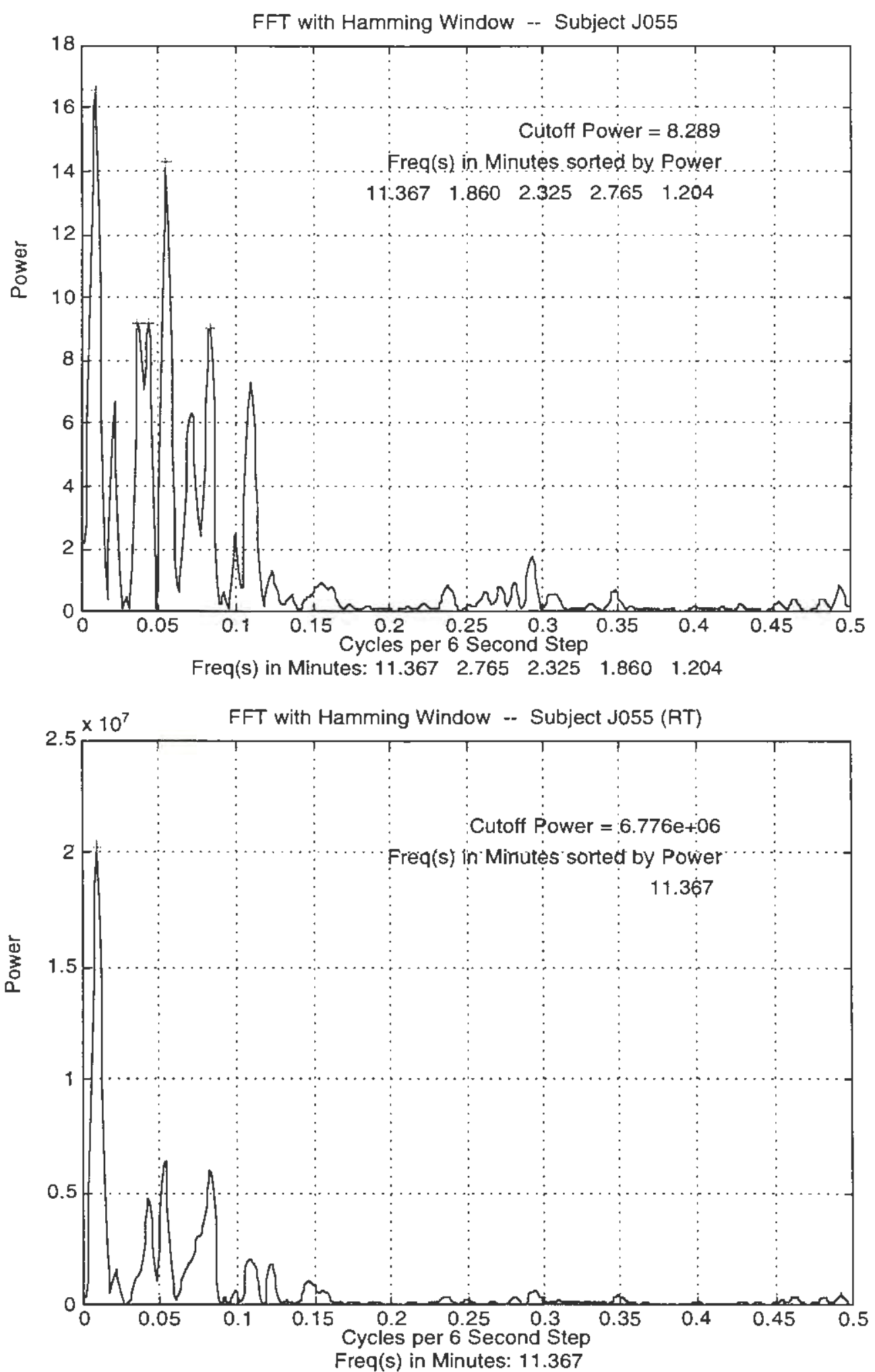

Figure 20. Power Spectra (Accuracy and RT) for Human Subject 55, rated as Different for primary frequency bands. 


\section{Bibliography}

Arruda, J.E. (1994). Confirmatory factor analysis of qualified electroencephalogram measured during a continuous performance test: A confirmation of Neurocognitive systems. Unpublished doctoral dissertation, University of Rhode Island, Kingston.

Arruda, J. E., Weiler, M. D., Valentino, D., Willis, W. G., Rossi, J. S., Stern, R. A., Gold, S. M., \& Costa, L. (1996). A guide for applying principalcomponents analysis and confirmatory factor analysis to quantitative electroencephalogram data. Manuscript submitted for publication.

Conte, S., Ferlazzo, P., \& Renzi, P. (1995). Ultradian rhythms of reaction times in performance in vigilance tasks. Biological Psychology, 39, 159-172.

Davies, D. R., \& Parasuraman, R. (1982). Decision theory. In P. Warr (Ed.). The Psychology of Vigilance (pp. 38-59). New York: Academic Press.

Gliden, D. L., \& Wilson, S. G. (1995a). On the nature of streaks in signal detection. Cognitive Psychology, 28, 17-64.

Gilden, D. L., \& Wilson, S. G. (1995b). Streaks in skilled performance. Psychonomic Bulletin \& Review, 2 (2), 260-265.

Gold, S. M. (1994). Quantitative EEG effects due to task related processing of lateralized auditory continuous performance tests, separate from attentional and motor components of the tasks. Unpublished doctoral dissertation, University of Rhode Island, Kingston.

Halperin, J. M., Sharma, V., Greenblatt, E., \& Schwartz, S. T. (1991). Assessment of the continuous performance test: Reliability and validity in a nonreferred sample. Psychological Assessment. 3, (4), 603-608. 
Halperin, J. M., Wolf, L. E., Pascualvaca, D. M.. Newcorn, J. H., Healey, J. M., O'Brein, J. D., Morganstein, A., \& Young, J. G. (1988) Differential assessment of attention and impulsivity in children. Journal of the American Academy of Child and Adolescent Psychiatry, 27, 326-329.

Mackworth, N.H. (1948). The breakdown of vigilance during prolonged visual search. Quarterly Journal of Experimental Psychology, 1, 6-21.

Makeig, S. \& Inlow, M. (1993). Lapses in alertness: coherence of fluctuations in performance and EEG spectrum. Electroencephalography and clinical Neurophysiology, 86 , 23-35.

Makeig, S. \& Jung, T. P. (in press). Tonic, phasic, and transient EEG correlates of auditory awareness in drowsiness. Cognitive Brain Research.

O'Dougherty, M., Nuechterlein, K. H., \& Drew, B. (1984). Hyperactive anf hypoxic children: Signal detection, sustained attention and behavior. Journal of Abnormal Psychology, 93, 178-191.

Oldfield, R. C. (1971). The assessment and analysis of handedness: The Edinburgh Inventory. Neuropsychologia, 9, 97-114.

Parasuraman, R (1984). The psychobiology of sustained attention. In J.S. Warm (Ed.), Sustained Attention in Human Performance (pp. 61-91). New Yory, NY: John Wiley \& Sons.

See, E. J., Howe, S. R., Warm, J. S., \& Dember, W. N. (1995). Metaanalysis of the sensitivity decrement in vigilance. Psychological Bulletin 117 (2), 230-249.

Shaprio, S. K., \& Garfinke, B. D. (1986). The occurrances of behavior disorders in children: The interdependence of attention deficit disorder and 
conduct disorder. Journal of the American Academy of Child and Adolescent Psychiatry, 25, 809-819.

Swanson, L. (1981). Vigilance deficit in learning disabled children: A signal detection analysis. Journal of Child Psychology and Psychiatry.22 393399.

Tucker, D.M. (1981). Lateral brain function, emotion, and conceptualization. Psychological Bulletin. 89(1), 19-46.

Tversky, A., \& Kahneman, D. (1971). Belief in the law of small numbers. Psychological Bulletin, 76 105-110.

Tversky, A., \& Kahneman, D. (1974). Judgement under uncertainty: Heuristics and biases. Science, 185 1124-1131.

Warm, J. S. (Ed.). (1984). Sustained attention in human performance. New York, NY: John Wiley \& Sons. 